\title{
HALIFAX, NOVA SCOTIA
}

\section{JUNE 12- 16, 2001}

\section{CCNS Executive Committee}

President: Alan Goodridge

Secretary-Treasurer: Mark Hamilton

Executive Vice-President: Robert G.Lee

Local Organizing Committee

Ivar Mendez

Allan Purdy

Diane Pottie

Scientific Program Committee

Chair: Wayne Martin

Timothy Benstead

George Elleker

Howard Feldman

J. Max Findlay

Mark Freedman

Ivar Mendez

Allan Purdy

Michael Shevell

Barry Sinclair

Jon Stoessl

Chris Wallace

Canadian Congress of Neurological Sciences

709 - 7015 Macleod Trail SW,

Calgary, AB Canada T2H 2K6

Tel: (403) 229-9544 • Fax: (403) 229-1661

E-mail: brains@ccns.org 36th MEETING OF THE CANADIAN CONGRESS OF NEUROLOGICAL SCIENCES
PROGRAM AND

ABSTRACTS
The Canadian Journal of Neurological Sciences does not assume any responsibility or liability for any errors in the abstracts.

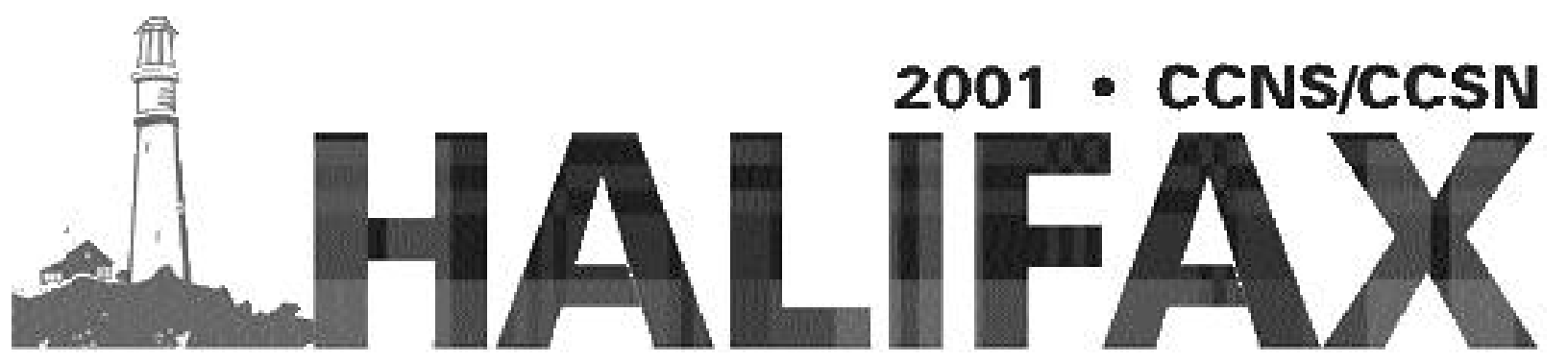




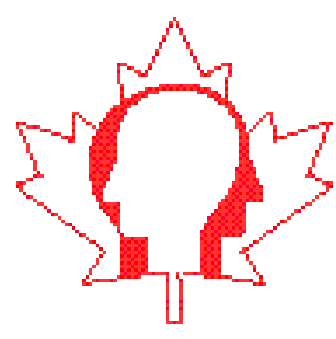

THE CANADIAN JOURNAL OF

Neurological Sciences

\section{LE JOURNAL CANADIEN DES Sciences Neurologiques}

Editor-in-Chief/Rédacteur en chef Douglas W. Zochodne CALGARY, AB

Associate Editors/Rédacteurs associés William A. Fletcher CALGARY, AB Andres M. Lozano TORONTO, ON

Past Editors/Anciens rédacteurs en chef James A. Sharpe TORONTO, ON Robert G. Lee CALGARY, AB Robert T. Ross WINNIPEG, MB (Emeritus Editor, Founding Editor)

Editorial Board/Conseil Scientifique Jack P. Antel MONTREAL, QC Timothy J. Benstead HALIFAX, NS J. Gregory Cairncross LONDON, ON Andrew A. Eisen VANCOUVER, BC J. Max Findlay EDMONTON, AB Anthony M. Hakim OTTAWA, ON Renn Holness HALIFAX, NS Alan C. Jackson KINGSTON, ON Douglas Kondziolka PITTSBURGH, PA, USA Mark J Morrow CLEVELAND, OH, USA Terence Myles CALGARY, AB John H. Noseworthy ROCHESTER, MN, USA C. Warren Olanow NEW YORK, NY, USA David Ramsay LONDON, ON Peter M. Richardson LONDON, UK Guy Rouleau MONTREAL, QC Shashi S. Seshia WINNIPEG, MB Paul Steinbok VANCOUVER, BC Jonathan A. Stoessl VANCOUVER, BC

\section{SECTION EDITORS/CONSEIL DE RÉDACTION}

Neuroimaging Highlight/Neuroimagerie Mark Hudon CALGARY, AB

William $\mathrm{Hu}$ CALGARY, AB

Neuropathological Conference/Conférence sur la neuropathologie

David Ramsay LONDON, ON

Book Review/Critiques de livres Warren P. Mason TORONTO, ON

News Editor/Rédacteur (nouvelles)

John W. Norris TORONTO, ON

Managing Director/Gérant directrice

Sally A. Gregg CALGARY, AB

Publications Committee/Comité de Rédaction

G. Bryan Young LONDON, ON

Owen Williams WINNIPG, MB

Joseph Chu ETOBICOKE, ON

Joseph Dooley HALIFAX, NS
The official journal of: / La Revue officielle de:

The Canadian Neurological Society

La Société Canadienne de Neurologie

The Canadian Neurosurgical Society

La Société Canadienne de Neurochirurgie

The Canadian Society of Clinical Neurophysiologists La Société Canadienne de Neurophysiologie Clinique

The Canadian Association of Child Neurology

L'Association Canadienne de Neurologie Pédiatrique

The permanent secretariat for the four societies and the Canadian Congress of Neurological Sciences is at/

Le secrétariat des quatre associations et du Congrès Canadien des Sciences Neurologiques est situe en permanence à:

709 - 7015 Macleod Trail SW, Calgary AB, Canada T2H 2K6,

The Canadian Journal of Neurological Sciences is published quarterly. The annual subscription rate is $\$ 70$ for members; $\$ 77$ for non-members in Canada; $\$ 88$ for USAand elsewhere. Residents, Interns, Pre- and Post-Doctoral Students $\$ 35$ per annum (members); $\$ 38.50$ per annum (non-members). Single copies $\$ 22$ each plus postage and handling. All manuscripts and communications should be sent to: Canadian Journal of Neurological Sciences, P.O. Box 5456, Station A, Calgary, AB Canada T2H 1X8. Courier to: 709 - 7015 Macleod Trail SW, Calgary, AB Canada T2H 2K6. Telephone (403) 229-9575; Fax (403) 2291661.E-mail: journal@cjns.org; Web Site: www.cjns.org COPYRIGHT@ 2001 by THE CANADIAN JOURNALOF NEUROLOGICAL SCIENCES INC. No part of this journal may be reproduced in any form without the prior permission of The Canadian Journal of Neurological Sciences. Mailed under Publications Mail Registration number 09824. Postage paid at Calgary, Alberta. This journal is indexed by Index Medicus, EMBASE Excerpta Medica and Current Contents - Clinical Practice and Life Sciences, Elsevier Biobase/Current Awareness in Biological Sciences, Biological Abstracts, Chemical Abstracts, Current Advances in Ecological Sciences, Dent.index, Industrial Medicine, Industrial Science Reviews, INIS Automind, Nutrition Abstracts, Science Citation Index, Weed Abstract.

Le Journal Canadien des Sciences Neurologiques est publié trimestriellement. L'abonnement annuel est de $70 \$$ pour les membres; $77 \$$ pour les non-membres au Canada; 88 \$ pour les Etats Unis et ailleurs. Internes, résidents, fellows pré et post doctoral: $35 \$$ par année (membres); 38,50 \$ par année (non-membres). Copie simple: $22 \$$ plus affranchissement et manutention. Toutes les communications et les manuscrits doivent être adressés à Journal Canadien des Sciences Neurologiques, P.O. Box 5456, Station A, Calgary, AB Canada T2H 1X8. Par courrier: 709 - 7015 Macleod Trail SW, Calgary, AB Canada T2H 2K6. Téléphone (403) 229-9575; Fax (403) 229-1661. E-mail journal@cjns.org; Web Site: www.cjns.org

DROITS D'AUTEUR@ 2001: THE CANADIAN JOURNALOF NEUROLOGICAL SCIENCES INC. Aucune partie de ce Journal ne peut être reproduite, sous quelque forme que ce soit, sans la l'authorisation du Journal Canadien des Sciences Neurologiques. Posté sous registration de poste-publications no 09824. Port payé à Calgary, Alberta. Le Journal est cité et indexé dans Index Medicus, EMBASE Excerpta Medica et Current Contents - Clinical Practice et Life Sciences, Elsevier Biobase/Current Awareness in Biological Sciences, Biological Abstracts, Chemical Abstracts, Elsevier Biobase/Current Advances in Ecological Sciences, Dent.index, Industrial Medicine, Industrial Science Reviews, INIS Automind, Nutrition Abstracts, Science Citation Index, Weed Abstract.

Advertising representative/Représentant de publicité:

Sally Gregg, Canadian Journal of Neurological Sciences 709 - 7015 Macleod Trail SW, Calgary, AB Canada T2H 2K6 Tel (403) 229-9575 Fax (403) 229-1661

E-mail: journal@cjns.org

Web Site: www.cjns.org

Printer/Imprimeur:

Sundog Printing Limited, 1311 Ninth Avenue SW, Calgary, Alberta T3C $0 \mathrm{H} 9$

ISSN 0317 - 1671 


\section{6th MEETING OF THE CANADIAN CONGRESS OF NEUROLOGICAL SCIENCES}

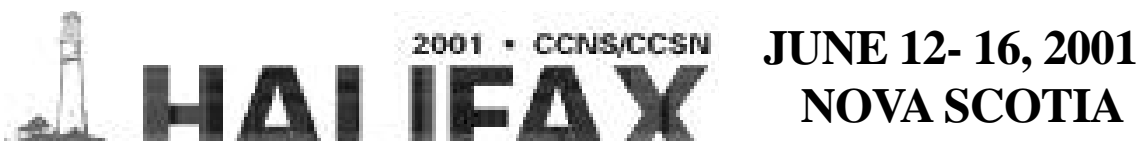

\section{Program ANd Abstracts}

\section{TuesdaY June 12, 2001}

\section{Neurobiology Review Course}

Chair: Garth Bray

An Update on the Autonomic Nervous System

Neural regulation of the cardiovascular system: Implications for neurological diseases .............. David A. Hopkins (Halifax, NS)

Clinical disorders of the ANS - Cardiovascular and other sequelae ........................ John D. Stewart (Montreal, QC)

Physiology and Pharmacology of Micturition

Neural circuitry and transmitters .... John W. Downie (Halifax, NS)

Clinical assessment and pharmacologic

manipulations

Jerzy Gajewski (Halifax, NS)

Learning and Memory from Different Perspectives

Neuroanatomy and

neuropsychology .................... Jeanette McGlone (Halifax, NS)

Cell and molecular biology .......... Wayne S. Sossin (Montreal, QC)

Eye Movement Control

The cerebellum and ocular motor control: Bringing physiology to the bedside ........... David S. Zee (Baltimore, USA)

The cerebrum and ocular motor control: Bringing the bedside to physiology and back ... James A. Sharpe (Toronto, ON)

\section{3rd Annual ALS Strategies for Quality \\ LifE/Quality CARE}

Chairs: Michael Strong, Suzanne Lawson

What is ALS? Timothy Benstead (Halifax, NS)

Nutritional intervention in

ALS ................................ Jeffrey Rosenfeld (Charlotte, USA)

Death and dying in ALS ........... Wendy Johnston (Edmonton, AB)

Panel Discussion: How the healthcare team can help

Michael Strong (London, ON), Wendy Johnston (Edmonton, AB) Colleen O'Connell (Fredericton, $N B$ )

Breakout groups: 1) Patients living with ALS,

2) Physicians: Management of respiratory complications,

3) Caregivers

The future of ALS Michael Strong (London, ON)

\author{
Unusual Movement Disorders Video Session \\ Chair: Doug Hobson
}

Case-based Session on Interesting Headaches

Chairs: Werner Becker, Allan Purdy

Course Presentations: Interesting headache cases

...... Werner Becker (Calgary, AB), Marek Gawel (Toronto, ON) ...Michel Aubé (Montreal, QC), Allan Purdy (Halifax, NS)

\section{WEDNESDAY JUNE 13, 2001}

\section{Common Movement Disorders: From the \\ Laboratory to The Clinic}

Chair: David King

Mechanisms of neurodegeneration in movement disorders Jon Stoessl (Vancouver, BC)

Genetics of movement disorders: Implications for treatment ........ David Grimes (Ottawa, ON)

Stem cell technology - Where we are and where we're going Ivar Mendez (Halifax, NS)

Neuroprotective agents in movement disorders: What's in the pipeline? ......... Mark Guttman (Markham, ON)

Office recognition of common movement disorders: a video review Mandar Jog (London, ON)

Growth factors and regenerative therapies in Parkinson's disease Janis Miyasaki (Toronto, ON)

Advances in Huntington's disease knowledge Oksana Suchowersky (Calgary, AB)

Dopamine agonists: New vs. old Warren Olanow (New York, USA)

Functional neurosurgery for movement disorders. What have we learned?

Surgery formovement disorders: Lessons from the operating room Andres M. Lozano (Toronto, ON)

Microelectrode recording during pallidotomy: essential, superfluous, or dangerous? Christopher Honey (Vancouver, BC) 


\section{Management of Lesions of The Cervicothoracic JUNCTION}

Chair: Michael G Fehlings

Anatomy/biomechanics of the cervicothoracic junction John Hurlbert (Calgary, $A B$ )

Anterior approaches to the cervicothoracic junction Michael Fehlings (Toronto, ON)

Posterior approaches to the cervicothoracic junction ...................... Richard Fox (Edmonton, $A B$ )

Fixation of the cervicothoracic junction Paul Marcotte (Philadelphia, USA)

Hands-on session \#1: Anterior cervical fixation (in sawbones)

Management of cancer of the cervicothoracic junction Ziya Gokaslan (Houston, USA)

Hands-on session \#2: Posterior cervical fixation (in sawbones)

\section{Lewy Body Dementia Course}

Chair: Michel Panisset

Diagnosis and investigation of Lewy body disease ......................... Michel Panisset (Montreal, QC)

Neuropathology of Lewy body disease ...................................... Yves Robitaille (Montreal, QC)

Cognitive aspects of Lewy body disease .. Morris Freedman (North York, ON)

Treatment of Lewy body disease Howard Feldman (Vancouver, BC)

\section{RECENT CONCEPTS IN ELECTROENCEPHALOGRAPHY}

Chair: Mark Sadler

Physiology of EEG Don Gross (Montreal, QC)

How to distinguish some abnormal EEG phenomena from similar but benign counterparts Warren Blume (London, ON)

Electrophysiology of myoclonus ...... Qais Ghanem (Ottawa, ON)

Subclinical status epilepticus in the intensive care unit G Bryan Young (London, ON)

EEG reading session/Quiz Mark Sadler (Halifax, NS)

\section{Mechanisms and Management of Neuromuscular DISEASE}

Chair: Timothy Benstead

Motor unit number estimation in

neuromuscular disease .................Ming Chan (Edmonton, $A B$ )

Myasthenia gravis ........................ Michael Nicolle (London, ON)

Sensory neuropathy .............................. Ian Grant (Halifax, NS)

Neuropathic pain .................... Douglas Zochodne (Calgary, AB)

\section{Controversies in Neurocritical Care}

Chair: Jeanne Teitelbaum

Anoxic-ischemic encephalopathy: prognostic determination G. Bryan Young (London, ON)

Vasospasm in subarachnoid hemorrhage: the latest in pathophysiology, detection, and management .................. J. Max Findlay (Edmonton, AB)

Mechanisms for the effect of sepsis? On the CNS and PNS: implications for management ....................... Charles Bolton (Rochester, USA)

Hypertension in critical neurological illness: should we be inducing hypertension in stroke? ............................... Philip Bath (Nottingham, UK)

Evidence for EEG monitoring in the ICU: effect on seizure detection and ICU length of stay Paul Vespa (Los Angeles, USA)

The role of hypertonic saline in intracranial hypertension ........ Mark Malkoff (Houston, USA)

\section{ThuRSDAY JUNE 14, 2001}

Meet the Expert Breakfast - Neurosurgery Charles Tator (Toronto, ON)

\section{Plenary Session I: Neural Transplantation}

Chairs: Alan Goodridge, Richard Moulton

Cell therapy for Parkinson's disease: current status and future perspectives .... Anders Björklund* (Lund, Sweden)

Fetal nigral transplantation in PD, where we have been and where we are going?.... Warren Olanow (New York, USA)

Neural transplantation for Parkinson's disease: New surgical strategies Ivar Mendez (Halifax, NS)

Finding new donor cells for Parkinson's disease: Xeno- or stem cells Ole Isacson (Belmont, USA)

\section{ExHIBITS/Posters}

\section{INDUSTRY COURSE}

The management of diabetic peripheral neuropathy Tim Benstead (Halifax, NS)

Epilepsy update Mark Sadler (Halifax, NS)

The impact of stroke units on stroke outcome Gord Gubitz, (Halifax, NS)

Advances in deep brain stimulation for the treatment of movement disorders Ivar Mendez (Halifax, NS)

\section{Oral Platform Sessions}

\section{Poster Sessions/Exhibits}

\footnotetext{
*Sponsored by the Royal College of Physicians and Surgeons of Canada
} 


\section{Plenary Session II: Regeneration/Plasticity}

Chairs: Alan Goodridge, Richard Moulton

Regeneration and repair in the peripheral nervous system .................. Douglas Zochodne (Calgary, AB)

The neurotrauma epidemic: prevention and regeneration issues Charles Tator (Toronto, ON)

CI therapy: Massive cortical reorganization in association with a large rehabilitation effect Edward Taub* (Birmingham, USA)

Abstract presentations

\section{FRIDAY JUNE 15, 2001}

\section{InFORMATION SESSion ON MAINTENANCE OF Certification}

Neurology Oksana Suchowersky (Calgary, $A B$ )

Neurosurgery Brian Wheelock (Saint John, NB)

\section{Plenary Session III: Neuroprotection}

Chairs: Alan Goodridge, Richard Moulton

Do motor neurons rust?The case for oxidation Michael Strong* (London, ON)

Neuroprotection in cerebral ischemia: Lessons learned from the failed clinical trials Ashfaq Shuaib (Edmonton, $A B$ )

Neuroprotection for the developing brain Michael Johnston* (Baltimore, USA)

Abstract presentations

\section{Exhibits/Posters}

\section{Oral Platform Sessions}

\section{Neurology Debate}

Chair: Alan Guberman

Resolved that we now have a rational basis for choosing pharmacotherapy (both mono- and poly-) in epilepsy

Jack Schneiderman (Toronto, ON) Kevin Farrell (Vancouver, BC)

\section{Neurosurgery Debate}

Chair: Michael Fehlings

Case scenario of low back pain with degenerative disc disease: fusion versus no fusion John Hurlbert (Calgary, $A B$ ) Paul Marcotte (Penn Valley, USA)

\section{SATURDAY JUNE 16, 2001}

\section{Multiple Sclerosis Course}

Chair: Dessa Sadovnick

MS overview including historical, immunology, pathogenesis

Natural history of MS ................. George C. Ebers (London, UK)

Differential diagnosis of MS, including MS in childhood Pierre Duquette (Montreal, QC)

Genetics of MS, including applications of findings for clinicians ......... Dessa Sadovnick (Vancouver, BC)

Symptomatic management of MS, including psychiatric and cognitive aspects .................... Joel Oger (Vancouver, BC)

Forensics 101 - Halifax edition .......... George Rice (Halifax, NS)

Disease modifying treatments .. William Pryse-Phillips (St. John's, NF)

Beyond modifying treatments: What to do if they are not effective, including the selection of innovative approaches Mark Freedman (Ottawa, ON)

Child Neurology Day:

Pediatric Neuro-oncology

Chair: Juliette Hukin

The psychosocial impact of pediatric brain tumours Ann Chapman (Halifax, NS)

The neurological complications of pediatric malignancies Juliette Hukin (Vancouver, BC)

The management of diencephalic tumours in childhood Jeffrey Allen (New York, USA)

FMRI and cortical mapping correlation and relevance in the resection of pediatric brain tumours William Logan (Toronto, ON)

Quacumque vera - Pursuing the truth in pediatric neuro-oncology Vivtek Mehta (Halifax, NS)

Molecular biology and its current and potential relevance in treatment strategies and prognosis of brain tumours in childhood ......... James Rutka (Toronto, ON)

The management of incidentally diagnosed tumours Jeffrey Allen (New York, USA)

*Sponsored by the Royal College of Physicians and Surgeons of Canada 


\section{Neuropathic Pain/Spinal Cord Injuries}

Full day course (with the Canadian Association of Physical Medicine and Rehabilitation)

Chairs: Christine Short, Michael Fehlings

Autonomic dysfunction:

Basic science Lynne Weaver (London, ON) Clinical aspects .... Robert Teasell, Keith Sequeria (London, ON)

Neurosurgical options for neuropathic pain Zelma Kiss (Calgary, AB)

DVT prevention and detection after spinal cord injury David Anderson (Halifax, NS)

SECTION 1: ACUTE InTERVEntions

Pathophysiology of spinal cord injury ... Charles Tator(Toronto, $O N)$

Role for acute surgery in spinal cord injury: rationale, evidence, state of the art ....... Michael Fehlings (Toronto, ON)

SECTION 2: Regeneration/TransPlantation

Strategies to overcome myelin inhibition to promote regeneration in the injured spinal cord ..... Sam David (Montreal, $Q C$ )

Bridging the injured spinal cord to facilitate regeneration Mary Bunge (Miami, USA)

Fetal cell transplantation to facilitate CNS regeneration Ivar Mendez, (Halifax, NS)

Spinal cord regeneration: a neurosurgeon's perspective Charles Tator (Toronto, ON)

\section{Stroke Course}

Chairs: Frank Silver, Christopher Wallace

Intracerebral hemorrhage: Classification and epidemiology Michael Hill (Calgary, $A B$ )

Hypertension in the community: Incidence, current treatment Timothy Dean (Halifax, NS)

ICH: triage, diagnosis, medical management Tom Currie

Surgery for ICH: Indications, results .............. David Mendelow (Newcastle-upon-Tyne, UK)

Deep ICH (basal ganglia); Amyloid-cerebral, lobar; Hematoma with SAH .................. Frank Silver (Toronto, ON) Christopher Wallace (Toronto, ON)

Canadian Stroke Network/Stroke Systems Stephen Phillips (Halifax, NS)

Decompressive craniotomy for ischemic stroke ................................. Andrew Demchuk (Calgary, AB)

Imaging for SAH ............................................................. TBA

Clinical/CSFdiagnosis of SAH - acute and delayed .......... TBA

Update management of ruptured aneurysms ..................... TBA

Strategies for treatment of giant aneurysms .......... M. Tymianski

Familial aneurysms when and how to screen .......................... D. Roy, J. Raymond

Incidental aneurysm patient - office decisions ............ P. Porter,

Asymptomatic aneurysms; CT-neg SAH; vertebral dissection

P. Porter, M. Tymianski 


\title{
36th MEETING OF THE CANADIAN CONGRESS OF NEUROLOGICAL SCIENCES

\author{
JUNE 12 - 16, 2001
} \\ NOVA SCOTIA
}

\author{
Abstracts \\ Prize Paper Presentations \\ Frances McNaughton Memorial Prize \\ President's Prize \\ K.G. McKenzie Prize in Clinical Neuroscience Research \\ K.G. McKenzie Prize in Basic Neuroscience Research

\section{Oral Platform Presentations} \\ Friday June 15, 2001 \\ F. Plenary Session III \\ $\mathrm{F}-01$ \\ G. Spinal \\ G-01 to G-04 \\ H. Epilepsy Surgery \\ $\mathrm{H}-01$ to $\mathrm{H}-03$ \\ I. Child Neurology \\ I-01 to $\mathrm{I}-07$ \\ J. General Neurology ...................... J-01 to J-07 \\ K. Neuro-oncology/Neuroscience ... K-01 to K-07
}

Thursday June 14, 2001
A. Plenary Session II
A-01 to A-02
B. Neurophysiology
$\mathrm{B}-01$ to $\mathrm{B}-04$
C. Movement Disorders $\mathrm{C}-01$ to $\mathrm{C}-03$
D. General Neurosurgery D-01 to D-07
E. Cerebrovascular Disease E-01 to E-07

\section{Poster Presentations}

Thursday June 14, 2001 and Friday June 15, 2001

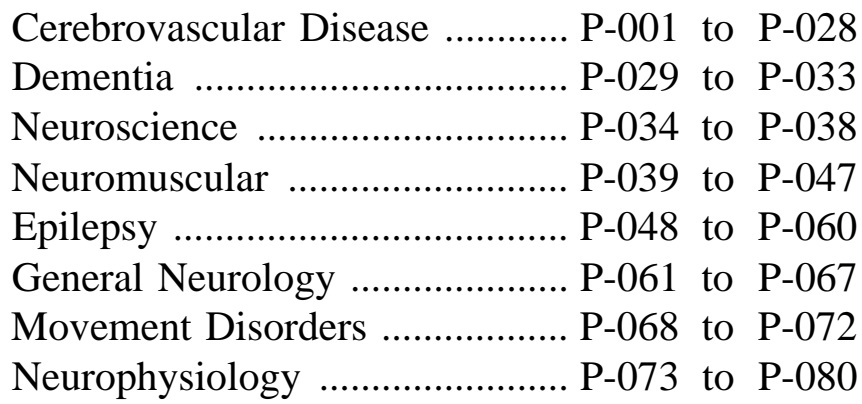

Neuro-oncology $\mathrm{P}-081$ to $\mathrm{P}-103$

Spinal Surgery $\mathrm{P}-104$ to $\mathrm{P}-114$

General Neurosurgery $\mathrm{P}-115$ to $\mathrm{P}-126$

Trauma $\mathrm{P}-127$ to $\mathrm{P}-131$

Peripheral Nerve $\mathrm{P}-132$ to $\mathrm{P}-134$

Pediatric Neurosurgery $\mathrm{P}-135$ to $\mathrm{P}-145$

Child Neurology P-146 to P-164 


\section{Prize Papers}

\section{Frances McNaughton Memorial PRIZE}

\section{Factors associated with early and late unemployment in multiple sclerosis patients}

\author{
K. Busche, L. Metz (Calgary, Alberta), J. Fisk (Halifax, \\ Nova Scotia)
}

Background: Unemployment in multiple sclerosis (MS) patients is associated with loss of income and impaired quality of life. Unemployment in MS is common. This study determined the demographic and disease related factors associated with unemployment at two time points in patients from the University of Calgary MS clinic.

Methods: Patients who provided clinical, demographic and employment data at both time points were eligible for inclusion. Variables associated with 'early' and 'late' unemployment were determined using contingency tables and Fisher's exact test. Odds ratios (OR) were calculated for each variable and stratified analysis was performed.

Results: At time 1, 49\% of 99 patients were unemployed. By time 2, 61\% were unemployed. Factors associated with an increased chance of unemployment at time 1 included: higher EDSS group, progressive course of disease, longer duration of disease, age greater than 39 years and male sex. Factors associated with unemployment between time 1 and time 2 included: higher EDSS group, age greater than 39 years and marital status (being married or cohabiting). No interactions or confounding factors were detected.

Conclusions: The unemployment rate in this cohort is high. Although the sample size was small, significant associations were detected, including previously unreported associations (male sex, marital status).

\section{The President's Prize}

Presumed pre- or perinatal arterial ischemic stroke: risk factors and outcomes

Meredith Golomb (Toronto, Ontario)

Background: A subgroup of children with arterial ischemic stroke within the pre- or perinatal period present with delayed diagnosis.

Methods: We identified 22 children who met the following criteria: (1) normal neonatal neurological history, (2) hemiparesis and/or seizures first recognized after two months of age, and (3) computed tomography or magnetic resonance imaging showing remote cerebral infarct. Laboratory evaluations included protein $\mathrm{C}$, protein $\mathrm{S}$, antithrombin, activated protein $\mathrm{C}$ resistance screen (APCR), Factor V Leiden (FVL), prothrombin gene defect, methylene tetrahydrofolate reductase variant (MTHFR), anticardiolipin antibody (ACLA), and lupus anticoagulant. Not all children received all tests. Age at last visit ranged from 8 months to 16.5 years (median four years).

Results: Twelve were male. Fourteen had left hemisphere infarcts. Median age at presentation was six months. Eighteen had gestational complications. Fourteen children had at least transient coagulation abnormalities (ACLA $=11$, ACLA+ $\mathrm{APCR}=1, \mathrm{APCR}=2$ with $\mathrm{FVL}+\mathrm{MTHFR}=1$ ); six of these children had family histories suggestive of thrombosis. Cardiac echocardiogram was unremarkable in the 12 tested. Outcomes were: speech, behavior, or learning problems in 12, persistent seizures in 5 , and persistent hemiparesis in 22 , with no evidence of further stroke in any patient.

Conclusions: The persistence and importance of coagulation abnormalities in this group need further study. 


\section{Prize Papers}

\section{The K.G. McKenzie Prize in Clinical NeUroscience RESEARCH}

\section{Risk factors for intracranial hemorrhage in full-term infants}

\section{B.S. Jhawar, A. Ranger (London, Ontario), R.F Del Maestro} (Montreal, Quebec)

Introduction: Few well-controlled studies have evaluated risk factors for intracranial hemorrhage (ICH) in full-term infants. In this study we set out to quantify the risk associated with forceps instrumentation, low platelet count and indicators of perinatal hypoxia for $\mathrm{ICH}$.

Methods: The study is based on a retrospective case-control design with matching on year-of-birth, sex and obstetrician. Cases were delivered at or transferred to the London Health Sciences Center between January 1, 1985 and December 31, 1996. All patients were evaluated with regard to mode of delivery, indicators of perinatal hypoxia (requirement for resuscitation and low Apgar scores) and coagulation status within 48 hours if available.

Results: Sixty-six cases and 104 controls were identified. Multivariate modeling of the following risk factors for $\mathrm{ICH}$ revealed an odds ratio of 4.3 (95\% CI, 1.2-15.0) for forceps instrumentation, 0.34 (95\% CI, 0.03-3.2) for cesarean section and 5.1 (95\% CI, 1.8-14.1) for the requirement for resuscitation. Lower Apgar scores at either one or five minutes were also associated with elevated risk for ICH. Of the 52 cases with available platelet counts, $30.8 \%$ (95\% CI, 18.3\%-43.3\%) had counts less than $70 \times 10^{9} / \mathrm{L}$.

Conclusion: In this study we identified several independent risk factors for $\mathrm{ICH}$. We found that thrombocytopenia, forceps instrumentation, and indicators of perinatal hypoxia (low Apgar scores and the requirement of resuscitation) were associated with increased risk for intracranial hemorrhage.

\section{K.G. McKenzie Prize in Basic NeUROSCIENCE RESEARCH}

Evidence for the role of FAS and P75 in post-traumatic
cell death and axonal degeneration and a neuroprotec-
tive effect of FAS gene deletion after spinal cord injury

\section{S. Casha, M.G. Fehlings (Toronto, Ontario)}

Introduction: Post-traumatic glial apoptosis contributes to axonal dysfunction and neurologic deficit after spinal cord injury (SCI). We examined the FAS and P75 death receptors pathways in apoptosis after SCI.

Methods: Adult rats underwent SCI at C7 (35g clip). FAS and P75 expression, and caspase 3 and 8 activation were examined by western blotting at $1,2,7$ and 14 days. The cellular distribution of FAS and P75, and colocalization with TUNEL was examined by immunohistochemistry. FAS -/- mice (and wildtype) were injured at T5 (8g clip). TUNEL was quantified at days 1,2 and 7 ( $n=3$ /group/timepoint). Western blotting was performed for FAS, caspase 8, caspase 3 and NF200. Behavioural recovery was assessed by the BBB scale for six weeks.

Results: In rat, FAS and P75 expression increased following SCI peaking at day 7. Oligodendrocytes and microglia showing evidence of post-traumatic apoptosis, colocalized with FAS and P75 and were spatially related to degenerating axons. NF200 was preserved following injury in FAS -/- mice indicating axonal preservation. Behaviourally, FAS -/- mice exhibited significant improvement in BBB scores.

Discussion: We demonstrate FAS and P75 initiate apoptosis after SCI and conclude that the FAS death receptor cascade is an attractive neuroprotective target in CNS injury. 


\section{Oral Platform Presentations}

\section{PLENARY SESSION II}

\section{A-01}

\section{Results of a randomized controlled trial of surgical versus medical therapy of temporal lobe epilepsy \\ S. Wiebe, W.T. Blume, J.P. Girvin, M. Eliasziw, for the EESTLE study group (London, Ontario)}

Background: The effectiveness and safety of epilepsy surgery has not been assessed in randomized-controlled-trials. This is important because non-randomized studies overestimate benefit and underestimate harm.

Methods: In a parallel-group randomized-controlled trial, 80 patients with temporal lobe epilepsy were assigned either to immediate evaluation and surgery $(\mathrm{n}=40)$, or to an existing oneyear waiting list with optimization of anticonvulsants $(n=40)$. Eligibility, best medical therapy and primary outcomes (seizure freedom) were assessed by blinded epileptologists. Secondary outcomes were seizure frequency, seizure severity, quality of life, morbidity, and mortality. All patients were followed for one year.

Results: At one year, the cumulative proportion of patients free of complex partial seizures was $58 \%$ and $8 \%$, respectively, in the surgical and medical groups $(\mathrm{p}<0.0001)$. The corresponding proportions for freedom from any seizures were $38 \%$ and $3 \%$, respectively $(\mathrm{p}<0.0001)$. Seizure frequency, and severity of residual seizures was lower in the surgical group. Quality of life was significantly better with surgical treatment $(\mathrm{p}<0.001)$. More surgical $(55 \%)$ than medical $(36 \%)$ patients were employed or studying.

Conclusions: Scientifically rigorous epilepsy-surgery randomized-controlled-trials are feasible and necessary. Although surgery was significantly superior, the net benefit was smaller in this randomized trial (35-50\%) than in published case series $(70-80 \%)$.

\section{A-02}

\section{Efficacy and morbidity of stereoelectroencephalography (SEEG) in 217 patients using CT or MRI based stereotactic technique}

\section{A. De Almeida, André Olivier, F. Quesney, F. Dubeau, P. Gloor, G. Savard, F. Andermann (Montreal, Quebec)}

Background: SEEG is based on the concept that intracranial electrodes, placed both on the cortical surface and in different depths of the brain, can provide a three dimensional view of the origin and spread of an epileptic focus. A solid hypothesis concerning the site to be studied, based on clinical and imaging findings, is essential for surgical planning (Bancaud and Talairach 1965). The purpose of this paper is to define the general efficacy and morbidity of SEEG using modern methods of image and particularize risks related to exploration of specific lobes of the brain.
Methods: All patients admitted between 1982 and 1999 who underwent either CT or MRI guided stereotactic electrode implantations by one surgeon (A.O.) were reviewed. The efficacy was assessed based on the information obtained by the procedure whether or not resective surgery was indicated.

Results: 217 patients underwent 224 implantations in 394 hemispheres. Altogether, 1548 epidural cortical electrodes (CE) (including ground and reference) and 1474 depth electrodes (DE) making up 3022 electrodes were implanted. Two abscesses and no hemorrhagic complications were encountered with the temporal group (407 CE and $897 \mathrm{DE}$ ); one abscess and three hematomas occurred in the frontal group (437 CE and $525 \mathrm{DE}$ ). All patients who developed hematoma had moderate to severe atrophy. One case of meningitis and four of scalp meningitis were encountered. All patients with hematomas and infections responded promptly to treatment and no permanent sequela was encountered. Twenty-six patients $(11.6 \%)$ presented some psychiatric disturbance during monitoring and needed psychiatric surveillance. Two patients presented neurological deficits during angiography in the early 1980s. One patient kept a mild hemiparesis and represents the only case of permanent sequela in the entire series. Regarding the efficacy of the procedure, the results of recordings led to an indication for surgery in 178 patients $(79.5 \%)$. In another 37 patients $(16.5 \%)$ the data obtained were sufficient to exclude surgical treatment as an option. SEEG did not provide enough information to make a surgical decision in only nine cases $(4.0 \%)$. Thus, the overall efficacy, i.e.the ability to make a decision for or against surgery, was of the order of $96.0 \%$.

Conclusions: SEEG is a useful technique to provide information regarding surgical option in patients who otherwise would be rejected as surgical candidates. With the technique employed, the overall morbidity has been low and the majority of patients have tolerated the procedure very well.

\section{NEUROPHYSIOLOGY}

\section{B-01}

Comparative value of somatosensory evoked potentials (SSEP), electroencephalography (EEG) and transcranial doppler (TCD) for detection of intraoperative cerebral ischemia

\section{Rowed, D. Houlden, L. Burkholder (Toronto, Ontario)}

Background: Electrophysiological methods of monitoring during carotid endarterectomy (CEA) are reliable but may overestimate clinically significant cerebral ischemia. Transcranial doppler (TCD) can detect emboli, the most frequent cause of new postoperative neurological deficits, but is often not recordable intraoperatively. The present study assesses the comparative value of these potentially complementary techniques.

Methods: One hundred and thirty-two adults were monitored 
during CEA under general anaesthesia by means of bilateral median nerve somatosensory evoked potentials (SSEP), 4 channel continuous electroencephalography (EEG) and middle cerebral artery (MCA) TCD.

Results: There were no failures of electrophysiological monitoring but TCD was successful in only $47 \%$ in the intraoperative environment. Rapid and persisting loss of SSEP occurred in two patients and was associated with new, persisting neurological deficit in both. TCD detected intraoperative emboli in $37 \%$, though only one of these patients experienced a new postoperative deficit.

Conclusions: Intraoperative hemodynamic ischemia associated with new neurological deficit is infrequent and is reliably detected by SSEP disappearance. TCD is not reliable intraoperatively. Intraoperative emboli detected by TCD are rarely associated with new postoperative deficits.

\section{B-02}

\section{The functional significance of the $\mathrm{N} 400$ component of event-related brain potentials \\ B. Maleki (Hamilton, Ontario), J.B. Debruille (Montreal, Quebec)}

Background: The aim of the present study was to investigate the functional significance of the N400 component of eventrelated potentials (ERP) by examining two recent theories, the semantic integration theory and the knowledge inhibition theory.

Methods: To tease these two theories apart, ERPs were recorded from 15 subjects that were presented with homographs and non-homographs and asked to decide whether each word was a homograph or not. The semantic integration theory predicts greater N400 amplitudes for homographs perceived as having several meanings, whereas the knowledge inhibition theory predicts greater amplitudes for homographs perceived as having only one meaning.

Results: Repeated-measures ANOVAs did not show any statistically significant differences between the N400 of these two conditions at any electrode site. It was found, however, that the N400 amplitude at the frontal electrode Fz was significantly larger than the $\mathrm{N} 400$ amplitude at the posterior electrode $\mathrm{Pz}(\mathrm{F}$ $(1.48,20.72)=5.84, \mathrm{p}<.05)$.

Conclusions: The results indicate that there is no direct evidence to support either theory of the N400 component of ERPs. Furthermore, the more frontal distribution of the N400 suggests a new pattern of activation in ERPs, which could be explained by the novel protocol used in this experiment.

\section{B-03}

\section{Chronic model for atypical absence seizures: studies of developmental and gender sensitivity}

\section{Persad, M.A. Cortez, O.C. Snead III (Toronto, Ontario)}

Background: Developmental treatment with AY-9944 (AY) induces atypical absence seizures. Whether male/female differences in severity of response to AY, is pre- or postpubescent is unknown.

Methods: Sixteen neonatal Long Evans hooded rats were treated with AY $(7.5 \mathrm{mg} / \mathrm{kg})$ or saline from postnatal day (PD) 2 to PD26. Two injection groups at PD5-11, PD11-17, PD17-23 and single injection groups at PD5 and PD11 were used to determine the minimum number of AY doses. Epidural electrodes were implanted at PD50 for quantification of slow spike and wave duration (SSWD) in female-AY (FAY) and maleAY (MAY) over one hour EEG recordings at PD55.

Results: AY induced SSWD (mean duration, seconds/hour \pm SEM) were more frequent in female $(1196 \pm 349)$ than males $(566 \pm 265)(\mathrm{p} \leq 0.05$, Student's t-Test). PD5-11 group $(830 \pm 204)$ vs. PD17-23 $(355 \pm 118) \quad(\mathrm{p} \leq 0.05$, student's t-Test). Single injection at PD5 produced $(732 \pm 147)$, PD11 (570 \pm 177$)$ compared to controls $(120 \pm 60)(\mathrm{p} \leq 0.05$ student's t-Test). FAYSSWD per seizure $(8 \pm 3)$ vs. MAY-SSWD $(4 \pm 3)(\mathrm{p} \leq 0.05)$. FAY number of seizures/h $(129 \pm 18)$ vs. MAY $(101 \pm 13)(\mathrm{p} \leq 0.05)$ student's t-Test.

Conclusions: Data indicate that the increased susceptibility of female to AY-induced atypical absence seizures emerges prepubescently.

\section{B-04}

Serotonin exacerbates spike-and-wave discharge (SWD) in the chronic model of atypical absence seizures

\section{E. Bercovici, M.A. Cortez, O.C. Snead III (Toronto, Ontario)}

Background: Parachlorophenylalanine (PCPA) exacerbates $\gamma$ hydroxybutyrate (GHB) induced absence seizures through a nonserotonergic mechanism (Epilepsia 1998;39(6):36). The effect of PCPA in the AY-9944 (AY) Model remains to be determined.

Methods: Eighteen adult male Long Evans hooded rats were implanted with epidural electrodes for one hour Video ECoG recordings after developmental AY treatment $(7.5 \mathrm{mg} / \mathrm{kg})$ or saline. Baseline, PCPA (150 mg/kg for three days), 5hydroxytryptophan (5-HTP) $(50 \mathrm{mg} / \mathrm{kg})$, carbamazepine (CBZ) (32 mg/kg), ethosuximide (ETO) (100 mg/kg) and Arabic Gum (AG) $(10 \%)$ pretreatment recordings were ascertained at postnatal day (PD) 50 for quantification of SWD duration and the brains were removed for 5-HT determination.

Results: PCPA decrease SWD (mean \pm SEM) from $360.83 \pm 70.31$ to $139.33 \pm 38.21$ seconds/hour $(\mathrm{p}<0.05)$. PCPAETO decreased SWD from $122.50 \pm 60.96$ to $49.17 \pm 9.88$ seconds/ hour ( $\mathrm{p}=0.357)$. PCPA-CBZ decreased SWDfrom 1941.80 \pm 209.78 to $808.88 \pm 343.61$ seconds/hour ( $\mathrm{p}=0.004$ ) (ANOVA). PCPA-AG increased SWD from $32.33 \pm 14.30$ to $42.00 \pm 16.54$ seconds/hour $(\mathrm{p}=0.696)$. 5-HTP increased SWD $(1860.80 \pm 209)$ compared to controls $(\mathrm{p}<0.05)$ (Student's t-Test).

Conclusions: Serotonin exacerbated SWD duration in the AY model and 5-HT depletion appears to protect against atypical absence seizures. Data indicate that serotonergic mechanisms are involved in AY induced atypical absence seizures. 


\section{MOVEMENT DISORDERS}

\section{C-01}

\section{Subsequent grafts can promote recovery in hemiparkinsonian rats with a sub-optimally-placed initial graft}

\section{K.A. Baker, M. Purdy, D. Sadi, K. Mukhida, I. Mendez (Halifax, Nova Scotia)}

Background: Optimal placement of dopaminergic grafts is likely crucial in maximizing clinical recovery in Parkinsonian patients. In this study, we assessed whether a subsequent graft may enhance functional recovery following sub-optimal positioning of an initial graft.

Methods: Rats with unilateral 6-hydroxydopamine lesions of the right nigrostriatal pathway were randomly divided into three groups, and received either 900,000 fetal nigral cells in the medial striatum only $(n=6)$ or 900,000 cells in both the lateral and medial striatum (1.8 million total), either simultaneously $(n=8)$ or sequentially, six weeks after the initial medial graft $(n=6)$. Amphetamine-induced rotational behaviour was used to assess behavioural recovery and immunohistochemistry for tyrosinse hydroxylase (TH) to assess graft survival.

Results: Rotational behaviour was significantly reduced in only simultaneous and sequential graft groups at nine and 12 weeks following transplantation. All animals had viable transplants. TH immunohistochemistry revealed significantly more cells within the subsequent graft than the initial graft. No significant difference in the total number of cells between sequential and simultaneous groups was observed.

Conclusions: These findings suggest that the survival of the subsequent graft may be enhanced by the initial graft and that transplantation of a subsequent graft may be used to enhance functional recovery in transplanted rats with poor graft viability or suboptimal positioning of an initial graft. These results may have important implications in optimizing clinical neural transplantation for Parkinson's.

\section{C-02}

\section{Preoperative cognitive functioning is not predictive of changes in motor, cognitive, mood, or quality of life functioning post-pallidotomy in patients with advanced Parkinson's disease}

\section{C.R. Honey, J.A.R. Carr, M. Sinden, J.S. Martzke (Vancouver, British Columbia)}

Background: Pre-operative dementia is widely considered a contraindication for pallidotomy despite no prospective studies to support this belief. We studied a cohort of patients to assess if pre-operative cognitive function correlated with postoperative changes in i) dyskinesia, ii) cognition, iii) mood, or iv) quality of life.

Methods: 24 Parkinsonian patients selected for unilateral pallidotomy were prospectively tested for dyskinesia (scale 0-4) and a battery of measures before and after surgery. Pre-operative dementia was assessed with the Dementia Rating Scale (DRS).
Postoperative changes in cognition were operationalized as a change in a cognitive composite score based on eight measures sensitive to fronto-striatal dysfunction (Global 8 Cognitive Score (G8CS)). Mood (POMS) and quality of life (SF-36) were also assessed. Linear regression analyses were performed to test for correlations between pre-operative DRS and postoperative changes in i) dyskinesia, ii) G8CS, iii) POMS, and iv) SF-36. Pre- and postoperative dyskinesia was compared with a paired ttest.

Results: There was a significant improvement in dyskinesia for the entire cohort ( $\mathrm{p}$ less than 0.001) but no correlation between pre-operative dementia (DRS) and postoperative improvements in dyskinesia $(\mathrm{r}=0.01)$. There was no significant correlation between pre-operative dementia and postoperative change in cognition $(/ \mathrm{r} /=0.083)$, $\operatorname{mood}(/ \mathrm{r} /=0.22)$, or quality of life $(/ \mathrm{r} /=0.28)$.

Conclusions: Pre-operative dementia status was unrelated to motor, cognitive, mood, or quality of life changes following unilateral pallidotomy. We recommend patients with mild dementia not be excluded from the potential benefits of pallidotomy.

\section{C-03}

\section{Genetic and clinical profile of the inherited ataxias in a movement disorders clinic population}

Oksana Suchowersky, Sarah Furtado, Lisa Graham, Peter Bridge (Calgary, Alberta), Shyamal Das (West Bengal, India)

Background: 1) To determine the prevalence of spinocerebellar ataxia (SCA) patients in the movement disorders population 2) to determine the number and type of genetic abnormalities seen.

Methods: All patients diagnosed with adult onset ataxia since 1988 through the Movement Disorders and associated Neurogenetics Clinics were identified, family history information was collected, and DNA testing done for SCA $1,2,3,6,7,8$, Freidreich's ataxia (FA) and DRPLA. Secondary causes were excluded.

Results: 65 patients were identified. 32 patients from 26 families had a clear cut autosomal dominant (AD) family history. Of these, one had SCA1, four had SCA2, five had SCA3, three had SCA6, and one had SCA8. The rest have tested negative todate. Four patients had an autosomal recessive (AR) pattern: two tested positive for FA. In the sporadic families, only one was positive for SCA 3. Age of onset was similar in the AD and sporadic groups, but much younger in the AR group (45 vs. 24 years).

Conclusions: 1) In our SCA patients, $49 \%$ had a clear AD inheritance pattern. Of these, $54 \%$ have an identifiable mutation 2) SCA3, SCA2, and SCA6 are the most common mutations in our populations 3) in sporadic cases, genetic testing rarely provides a specific diagnosis. 


\section{GENERAL NEUROSURGERY}

\section{D-01}

\section{Risk factors for intracranial hemorrhage in full-term infants}

\section{B.S. Jhawar, A. Ranger (London, Ontario), R.F Del Maestro (Montreal, Quebec)}

Winner of The K.G. McKenzie Prize in Clinical Neuroscience Research (See page 9)

\section{D-02}

\section{Sustained effectiveness of the Think First for Kids program}

M.D. Cusimano, U. Ramanathan, D. Isaac, J. Li, M. Chipman, A. Sharman, E. Mansfield (Toronto, Ontario)

Background: The Think First for Kids Program (TFFK) is an injury prevention curriculum for children in grades 1,2 , and 3. Our previous work has shown that the program can have short term beneficial effects. This study evaluated the effectiveness of the program six months after its delivery.

Methods: An interrupted time series with an equivalent control group design was used. In the 1999 Fall semester, knowledge and behaviour tests were given to 584 "active" students in 27 classrooms before (T0) and after (T1) 8 week implementation of TFFK. 596 control students who did not receive TFFK did the same tests. All students were tested again six months after (T2) the first post-test. T-tests were used to analyze mean difference scores.

Results: Both groups showed equivalent baseline scores over the three grades. Test-retest reliability of the outcome measures was 0.7 . "Active" students showed significantly better scores than "monitoring" students. These results persisted at the six month test point (grade $1 \mathrm{p}=0.0003$; grade $2 \mathrm{p}<0.0001$; grade 3 $\mathrm{p}=0.0003$ ).

Conclusions: Elementary school children who receive TFFK show improved knowledge that persists for at least six months after receiving the program. Future work will focus on the relationships of knowledge, intentions and injury events.

\section{D-03}

\section{All terrain vehicle related trauma in New Brunswick}

\section{S. Walling, H. Oakley J. Ducharme (Saint John, New} Brunswick)

Introduction: All terrain vehicle (ATV) related trauma continues to be a serious concern for health care providers. It has been our impression that the magnitude of the problem has not been fully assessed or brought to the attention of the public and governmental policy regulatory boards. The purpose of this review is to describe the nature and severity of head and spine injuries sustained from ATV accidents. We will also attempt to identify the associated risk factors for severe injury requiring a hospital visit or admission to a major trauma center from March 1998 to April 2000.
Methods: A retrospective review was done of all patients admitted with injuries related to ATV accidents over a 24 month period at the Saint John Regional Hospital in Saint John, New Brunswick (a tertiary care university hospital).

Results: A total of 106 cases were reviewed. The mean age was 32.8; the male-to-female ratio was $8.1 ; 13 \%$ did not wear helmets; and alcohol use was involved in $21.6 \%$ of the accidents. The mean Injury Severity Score (ISS) of those who wore a helmet without consuming alcohol was 13.08; the mean ISS of those without a helmet was 16.5; and those who were not wearing a helmet and consumed alcohol had a mean ISS of 17.9. There were two spinal cord injuries and 20 head injuries, nine of which had an admission Glasgow Coma Scale of less than 8 . There were 45 Intensive Care admissions with four deaths after admission. The total number of reported ATV accidents by the RCMPwas 167 with nine deaths province wide.

Conclusion: ATV use in New Brunswick continues to be a source of major morbidity and mortality, raising the questions whether the safety regulations, driver education, licensing and public awareness should be reassessed.

\section{D-04}

Reoperation for acute hemispheric stroke following carotid endarterectomy: is there any value?

\section{J. Max Findlay (Edmonton, Alberta)}

Background: Since the clinical benefit of urgent investigation and carotid rerepair for acute stroke complicating carotid endarterectomy (CEA) is uncertain, the results of this approach were examined in a large series of patients.

Methods: In a consecutive series of 700 CEAs, 24 patients (3.4\%) suffered a postoperative stroke. Twelve of these 24 patients experienced major hemispheric deficits (hemiplegia + /aphasia, forced eye deviation and decreased consciousness) that prompted either immediate surgical reexploration or cerebral angiography with reoperation based on angiogram results; those 12 patients are the subject of this report. Neurological improvement was attributed to carotid reopening when affected muscle strength increased to anti-gravity power within six hours of reoperation.

Results: Of the 12 patients with severe postoperative deficits, five $(42 \%)$ were present upon awakening, six occurred within 12 hours of surgery and the only intracerebral hemorrhage in this series occurred eight days after surgery. Four patients underwent urgent reoperation without angiography, and in two, carotid occlusions were found and repaired. In another the carotid was patent, and an intra-arterial injection of rt-PA $(20 \mathrm{mg})$ was given. Of the seven patients that underwent cerebral angiography as the first step, two carotid occlusions and two residual stenoses with attached thrombus were found and urgently repaired. Surgical reopening of occluded arteries improved in two of four patients, and early improvement was noted in one patient with a stenosis correction and the patient who received intraoperative rt-PA. Three patients who underwent urgent reoperation did not show benefit early after surgery. One patient died, two were left with major deficits, five moderate, and four patients eventually made good recoveries at a minimum of six months follow-up.

Conclusions: In this series, urgent carotid rerepair for major 
hemispheric strokes complicating CEA led to early neurological improvement in about one-half of patients. An aggressive approach to the catastrophe of postCEA hemiplegia minimizes ischemic damage in some patients.

\section{D-05}

\section{Should ICU patients in traction be mobilized in a halo vest?}

S.D. Christie, D.B. Clarke, D. Morrison, G. Rocker (Halifax, Nova Scotia)

Background: Based on the idea that the halo vest itself provides a significant restrictive component to pulmonary function, patients with cervical spine instability that require mechanical ventilation are commonly placed in traction and nursed in the supine position until extubated. This contrasts with the critical care literature, which advocates the sitting position for improved pulmonary toilet in ventilator dependent patients. The aim of this study is to assess, in healthy volunteers, the degree to which halo vest immobilization affects pulmonary function and to identify other factors that could negatively affect pulmonary function. Clinical significance, based on asthma studies, was set at $15 \%$.

Methods: Bedside spirometry was used to test healthy volunteers before and after application of the halo vest in both the sitting and supine positions. Demographic data collected included age, sex, smoking status and prior pulmonary disease.

Results: 25 subjects (12 male, 13 female) were investigated. The mean age was 31.4 years (range 21-54). Six people were smokers and eight had asthma. Although the halo was found to cause a statistically significant decrease in vital capacity both in the sitting $(-9.64 \% ; \mathrm{p}<0.000001)$ and the supine $(-8.07 \%$; $\mathrm{p}<0.000001)$ positions, these changes did not reach clinical significance. In addition, females demonstrated a greater decrease $(-11.3 \%)$ than males $(-4.6 \%)$ in the supine position $(\mathrm{p}=0.02)$.

Conclusion: Halo vest immobilization does not yield a clinically significant decrease in vital capacity, calling into question the current practice of nursing ventilator dependent patients supine and in traction. Being of female sex is associated with a greater decrease in vital capacity in the supine position only.

\section{D-06}

\section{Multimodal treatment of basal ganglia and thalamus arteriovenous malformations}

I.G. Fleetwood, M.P. Marks, M. Marcellus, G.K. Steinberg (Stanford, California, USA), R.P. Levy (Loma Linda, California, USA)

Background: Patients with high-flow arteriovenous malformations (AVMs) of the thalamus and basal ganglia develop progressive neurological deficits, intracranial hemorrhages $(\mathrm{ICH})$, intractable headaches and seizures and are difficult to manage.

Methods: Data collected prospectively from 1986 to the present was reviewed.
Results: Seventy-one patients (55\% male) were treated and average age at diagnosis was 16.8 years. Most presented with ICH $(75 \%)$. The mean nidus size was $3.65 \mathrm{~cm}$ and the median Spetzler grade was IV. Patients were treated with an average of 2.8 sessions (range $1-10$ ). Thirty-five microsurgical procedures, four surgical embolizations, 85 endovascular embolizations and 65 radiosurgery treatments were performed. The average size reduction per treatment with microsurgery, embolization and radiosurgery was $47.5 \%, 24.5 \%$ and $34 \%$, respectively, while the permanent complication rate was $12.7 \%$. Of cured patients, $72 \%$ required multiple treatment sessions and $44 \%$ required multimodal therapy. Five patients died before cure including four that developed ICH.

Conclusions: AVMs in this region affect young patients and have an aggressive course. Their treatment must find a balance between obliterating the nidus and producing new deficits. Staged multimodal treatment can produce good outcomes or cure patients with a morbidity and mortality rate that is acceptable, given the natural history of these lesions.

\section{D-07}

A cost analysis: the treatment of chronic pain by spinal cord stimulation versus alternative therapies

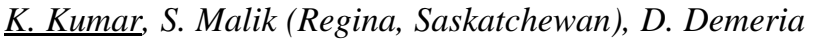
(Saskatoon, Saskatchewan)

Background: The use of spinal cord stimulation (SCS) for the treatment of chronic benign pain syndromes is an accepted treatment modality in a select group of patients. However, there are little data to measure the cost-effectiveness of SCS in comparison to conventional chronic pain therapy (CPT).

Methods: We present our series of 104 patients suffering from failed back syndrome. Of this group, 60 underwent spinal cord implantation, while 44 were treated with CPT and acted as controls. These patients have been followed for a 5-year period. The cost calculations for each group included imaging investigations, professional costs, implantation costs, nursing and physician visits, physiotherapy, chiropractic therapy and other alternative therapies and hospitalization costs for breakthrough pain. From these data, the cumulative costs for each group were calculated for a five-year period.

Results: The cumulative costs for SCS therapy for five years is $\$ 33,827$ as compared to CPT, $\$ 36,338.91$. The costs of treatment for the SCS group are greater than those of the CPT group in the first two years. From the third year on, SCS costs are lower than the CPT group.

Conclusions: SCS is costly within the first two years of treatment, after which the expenses of treating the CPT group continue to rise. More patients are able to return to gainful employment with SCS therapy than with CPT. 


\section{CEREBROVASCULAR DISEASE}

\section{E-01}

\section{Time course in cervical arterial dissection}

V. Beletsky (Toronto, Ontario), A. Shuaib (Edmonton, Alberta), A. Woolfenden (Vancouver, British Columbia), J.W. Norris (Toronto, Ontario) for the Canadian Stroke Consortium

Background: Cervical arterial dissection is one of the leading causes of stroke in the young. Role of trauma in dissection is underestimated, especially since symptoms may be delayed and traumatic cases are often judged as "spontaneous".

Material and methods: The Canadian Stroke Consortium is undertaking a prospective national study of carotid (CA) and vertebral artery (VA) dissections, including detailing trauma, concurrent familial disease (e.g. Marfan) and other features. All cases have angiography.

Results: In 108 patients (m/f:58/50; mean age 43.9, range 1687 ) there were 70 VA (5 spontaneous, 49 traumatic, 16 inadequate history) and $38 \mathrm{CA}$ (4-17-17 correspondingly) dissections. "Violent" trauma (e.g. neck manipulation) caused 21 dissections in the VA and six in CAgroup; other traumatic cases were related to trivial trauma (e.g. abrupt head turn, heavy lifting, various accidents). The median time between trauma and clinical presentation was 24 hours (range: minutes - 49 days in VA and minutes - 25 days in CA group). On angiography one patient had a potential source of emboli from a pseudo aneurysm nine months after the original trauma. Eleven patients $(10 \%)$ had recurrent neurological deficit within two weeks after trauma.

Conclusions: Although most of ischemic cerebral events occur within 24 hours, they may happen months afterwards due to permanent vascular abnormalities consequent to dissection.

\section{E-02}

\section{Pravastatin improves endothelial function in insulin resistant carotid arteries}

Aaron S. Dumont, Neal F. Kassell (Charlottesville, Virginia, USA), M. Eric Hyndman, Garnette R. Sutherland (Calgary, Alberta), Randall J. Dumont (Vancouver, British Columbia), Gideon Cohen, Subodh Verma (Toronto, Ontario)

Background: Insulin resistance and hypertension are independent risk factors for stroke. Endothelial dysfunction (in response to risk factors) and carotid artery disease are important in the pathogenesis of stroke. Pravastatin may have cholesterolindependent pleiotropic effects. The present study examined the effects of short-course pravastatin treatment on endothelial function in carotid arteries from control and fructose-induced insulin resistant rats.

Methods: Thirty rats were divided into two experimental groups. Fourteen were fed a regular diet and 16 were fed a fructose-enriched diet for three weeks. The rats were then divided into four groups: Control [C], Pravastatin treated Control [CP], Fructose fed [F], and Pravastatin treated Fructose fed [FP]. Pravastatin was administered (20mg/kg/day) for two weeks.
Urinary $\mathrm{NO}$ metabolite $\left(\mathrm{NO}_{2}^{-}\right)$excretion was also assayed. The carotid arteries from all rats were subsequently removed and assessed for endothelium-dependent and -independent vascular reactivity in vitro.

Results: F rats were insulin-resistant, hyperinsulinemic and hypertensive relative to $\mathrm{C}$ and $\mathrm{CP}$ rats and exhibited diminished endothelium-dependent vasomotion and urinary $\mathrm{NO}_{2}^{-}$- excretion $(\mathrm{p}<0.05)$ with preserved endothelium-independent vasomotion. Strikingly, pravastatin treatment restored endotheliumdependent vasomotion and urinary $\mathrm{NO}_{2}{ }^{-}$excretion in FP relative to $\mathrm{F}$ rats $(\mathrm{p}<0.05)$.

Conclusions: We report, for the first time, that pravastatin restores endothelial function in carotid arteries from insulin resistant fructose-hypertensive rats. These beneficial effects were ascribed to direct, cholesterol-independent vascular effects of pravastatin and likely result from augmentation of NO production. These data provide impetus for further investigation of non-lipid lowering indications for pravastatin therapy in the prevention and treatment of carotid artery disease.

\section{E-03}

\section{Acute IV-IA revascularization therapy for severe ischemic stroke}

Michael D. Hill, Philip A. Barber, Andrew M. Demchuk, Nancy J. Newcommon, Andrea Cole-Haskayne,

Karla Ryckborst, Laurel Sopher, Allison Button, William Hu, Mark E. Hudon, William Morrish, Richard Frayne, Alastair M. Buchan (Calgary, Alberta)

Background: Intravenous alteplase for acute ischemic stroke is least efficacious for patients with proximal large artery occlusions and clinically severe strokes. Intra-arterial therapy has the theoretical advantage of establishing a neurovascular diagnosis and high symptomatic artery patency rate, but is disadvantaged by the extra time and technical expertise required. A combination of these two approaches may provide the best chance of improving outcome in severe acute ischemic stroke.

Methods: Sequential patients arriving to our centre within three hours of stroke onset who were treated with intravenous alteplase, were screened for possible additional intra-arterial therapy using noninvasive neuroimaging. Clinical measures and outcomes were recorded prospectively.

Results: Nine patients over 24 months underwent a combined IV-IA approach. Seven received intra-arterial alteplase, one underwent intracranial angioplasty and in a final patient, technical aspects prevented IA therapy. Early neurovascular and/or neurometabolic imaging identified the location of occlusion and tissue-at-risk in all eight patients. Several practical problems were encountered. Two patients had a poor outcome and there were no instances of symptomatic intracerebral hemorrhage.

Conclusions: Intravenous followed by intra-arterial therapy is a promising approach to the treatment of severe acute ischemic stroke. There are several practical issues that must be solved to minimise the time to recanalisation of the symptomatic artery. Early noninvasive neurovascular and neuro-metabolic imaging is very helpful in choosing candidates for this type of therapy. 


\section{E-04}

\section{The Canadian Activase for Stroke Effectiveness Study (CASES)}

Michael D. Hill, Alastair M. Buchan (Calgary, Alberta) on behalf of the CASES Investigators

Background: Therapy for acute stroke using rtPA was approved in Canada in February 1999. The Canadian Activase for Stroke Effectiveness Study Group was formed to study the use of rtPA in Canada in a 30-month post-marketing study.

Purpose: To both prospectively assess the safety of rtPA in the Canadian context and to examine whether the efficacy of rtPA for acute stroke, demonstrated in the NINDS trial, can be translated into effectiveness in routine clinical practice across Canada.

Methods: The CASES group is a collaboration among the Canadian Stroke Consortium (CSC), the Canadian Stroke Society, the Heart and Stroke Foundation of Canada, HoffmannLa Roche Canada and physicians across the country. The study protocol has been approved by the research ethics board at each centre. Demographics, stroke risk factors, treatment factors and outcomes are being collected prospectively. Outcomes will be monitored at discharge and at three months.

Results: To date, 784 patients have been treated from 56 centres. The median NIHSS score is 14 (range 2-37). $29 \%$ of patients have minimal or no neurological deficit (NIHSS 0-1) and $45 \%$ are independent (mRS 0-2) at 90 days. The symptomatic hemorrhage rate is $4.5 \%$ (95\% CI $3.1 \%$ to $6.2 \%$ ). The rate of protocol violations is $13 \%$, the majority of which are time window violations. Based upon a phone survey of hospitals in Canada with CT scanners, the data set is $75 \%$ complete.

Conclusions: CASES is an ongoing prospective evaluation of the effectiveness of rtPA in acute stroke. The symptomatic hemorrhage rate is low (4.5\%). Alteplase for ischemic stroke is safe and effective in Canada.

\section{E-05}

\section{Why we don't treat acute stroke}

M.D. Hill, A.M. Buchan (Calgary, Alberta), N. Newcommon, S. Phillips, G. Gubitz, J. Jarrett, P. Stys, B.A. Schwarz, V. Hachinski, M. McTaggart, S.E. Black, Na Jiang, for the CASES Investigators

Background: Only a minority of patients are eligible for thrombolytic treatment of acute ischemic stroke. We sought to understand the reasons why patients who present to hospital with stroke symptoms within three hours of symptom onset are deemed ineligible for therapy.

Methods: Five centres, chosen from the CASES Investigators, volunteered to collect data prospectively for one year. Patients who presented to hospital with stroke symptoms within three hours of onset were eligible for entry into the registry. Demographics, stroke severity and reasons for not treating with thrombolysis were collected. The $24 \mathrm{~h}$ NIHSS scores were recorded.

Results: 345 patients were enrolled. The median age was 72 . $55 \%$ of patients were male. The median NIHSS was 3 (range 0-
34). The median onset-to-ER arrival time was 82 minutes and the median door-to-CT time was 40 minutes. In order of importance, the commonest reasons for not treating were: rapidly improving $(27 \%)$, deficit too mild (16\%), hemorrhage (12\%), suspected SAH (6\%), early ischemic changes on CT or MR too severe (5\%), other $(34 \%)$. Of the patients that were judged to be "rapidly improving", 55\% had a 24h NIHSS 0-1, 36\% had a $24 \mathrm{~h}$ NIHSS $2-5$ and $19 \%$ had an NIHSS $>5$. At discharge $13 \%$ of these patients were dependent (mRS 3-5).

Conclusions: The commonest reason for exclusion from therapy is the observation of a rapidly improving deficit. Despite this, one fifth of patients had a significant neurological deficit at $24 \mathrm{~h}$. At discharge, $13 \%$ of these patients remain dependent.

\section{E-06}

\section{Quantitative cerebral perfusion in a patient with chronic cerebrovascular disease}

M.J. Hogan, V.F. DaSilva, P. Kalapos, P. Healey (Ottawa, Ontario)

Background: Cerebral blood flow (CBF) thresholds for ischemic brain injury have been well-defined but the application of this knowledge to the management of patients with cerebrovascular disease has been limited. With the development of computerized tomography (CT) bolus tracking methods, quantitative CBF measurements may now easily be performed using standard CT. We describe CBF measurements in the evaluation of a patient with chronic cerebrovascular disease.

Methods: A 64-year-old woman with a past history of ischemic stroke presented with left eye pain and progressive visual loss. Bilateral carotid artery occlusions were identified although the distal left internal carotid (ICA) was found to be patent. Magnetic resonance imaging demonstrated infarct in a watershed distribution in the right cerebral hemisphere and more widespread subcortical ischemic changes. Quantitative cerebral perfusion measurements were obtained both before and after revascularization of the left internal carotid artery by endarterectomy. Bolus tracking CT measurements were obtained on a GE Medical Systems Hi-Speed scanner. Following a noncontrast scan, $0.5 \mathrm{ml} / \mathrm{kg}$ of Omnipaque 300 were administered intravenously at a rate of $4 \mathrm{ml} / \mathrm{sec}$. $40 \mathrm{CT}$ images were obtained every second at a selected transverse level. Cerebral blood volume (CBV), $\mathrm{CBF}$ and mean transit time (MTT) maps were calculated using a GE Advantage Workstation. These images were partitioned using a template to define cortical and subcortical structures. Large vessels were identified and excluded from the analysis.

Results: CBF ranged from 15 to $36 \mathrm{ml} / 100 \mathrm{~g} / \mathrm{min}$ in the right cerebral hemisphere cortical regions and 22 to $37 \mathrm{ml} / 100 \mathrm{~g} / \mathrm{min}$ on the left side with the lowest values in the frontal watershed zone. Within infarcted brain, cortical and white matter $\mathrm{CBF}$ values were 15 and $7 \mathrm{ml} / 100 \mathrm{~g} / \mathrm{min}$ respectively. Following left carotid endarterectomy CBF increased in the left hemisphere by $40 \%$ and mean transit time decreased by $40 \%$. In the contralateral cerebral hemisphere CBF decreased by $14 \%$ following endarterectomy compared to a $7 \%$ decrease in cerebellum and mean transit time increased by more than $40 \%$ in both of these regions. 
Conclusions: Endarterectomy improved perfusion to the left cerebral hemisphere while flow decreased slightly within the contralateral cerebral hemisphere. This study demonstrates the feasibility and utility of performing quantitative cerebral perfusion measurements in the assessment and management of patients with severe cerebrovascular disease.

\section{E-07}

\section{Systematic volume measurements of the acute ischemic stroke lesions}

Anna Tomanek, Ross Mitchell, Phillip Barber, Andrea ColeHaskayne, Alastair M. Buchan (Calgary, Alberta)

Background: The availability of diffusion weighted MR has given us a potential opportunity to understand the mechanisms involved in the evolution of acute ischemic stroke (AIS) by undertaking multiple serial imaging studies.

Methods: Between August 25, 1999 and January 16, 2000, 35 patients aged 34-85 (average 65.5, SD 12.8) with AIS were serially scanned using a 3 tesla MRI system. Patients were imaged on presentation (average delay of the scan from the symptom onset was 3.9 hours, range $2.0-7.8$ hours, SD 1.4), 24 hours later, between four and seven days and finally at 30 days. 33 patients participated in four serial studies and two patients in three studies. 11 patients were treated with IV tPA before or during the first MRI scan. NIHSS score was performed before or immediately after the MRI study. Our stroke protocol included T1, DWI, FLAIR, T2, MRAand PWI sequences. Volumes of the lesions were calculated using computer assisted technique previously developed and verified for quantifying MS lesion volumes. For volume calculations on days 0,1 , and 4-7 combined DW and FLAIR images were used, while for day 30, combined FLAIR and T2 images.

Results: 35 patients with median NIHSS of 8.5 (range 1-24) were included in the serial studies. Preliminary data shows a trend of growing lesions from the day 0 (average $9.5 \mathrm{cc}, \mathrm{SD}$ 16.0), day 1 (average $25.7 \mathrm{cc}, \mathrm{SD} 36.5$ ) to days $4-7$ (average $31.2 \mathrm{cc}, \mathrm{SD} 41.1$ ), and decrease in volume by the day 30 (average $22.1 \mathrm{cc}$, SD 31.8).

Conclusions: We have developed a simple computerized system to calculate lesion volumes. Our preliminary data suggests a trend towards growth of infarct volume.

\section{PLENARY SESSION III}

\section{F-01}

Evidence for the role of FAS and P75 in post-traumatic cell death and axonal degeneration and a neuroprotective effect of FAS gene deletion after spinal cord injury

\section{S. Casha, M.G. Fehlings (Toronto, Ontario)}

Winner of the K.G. McKenzie Prize in Basic Neuroscience Research (see page 9)

\section{SPINAL}

\section{G-01}

Prospective multicenter evaluation of the Codman semiconstrained anterior cervical plating system in 195 cases: long-term evaluation of clinical and radiological outcomes with quantitative evidence for load sharing

Michael G. Fehlings, Steven Casha (Toronto, Ontario)

Introduction: Semi-constrained load sharing implants for spinal fixation allow change in the screw-plate interface as bone grafts shrink. We evaluated the clinical and radiological outcome of the Codman anterior cervical plating system, which allows change in the screw-plate angle.

Methods: A 10 center prospective study with independent, blinded evaluation. All patients underwent cervical fusion with the Codman plate. Clinical and radiological evaluation was performed at 1, 3, 6, 12 and 24 months. Radiographs were examined for screw angles, construct height, fusion, and screw fracture or displacement.

Results: 195 patients were enrolled. Mean follow-up was 17.0 months. Neurological improvement was seen in $74.9 \%$, and pain improvement in $74.8 \%$. Fusion was successful in $93.8 \%$. Hardware failure occurred in $10.4 \%$; reoperation was required in 4 cases. A significant change in screw angles occurred over time (mean $=7.8^{\circ}$ in caudal screw angle, $\mathrm{p}<0.001 ; 2.1^{\circ}$ in the rostral screw angle, $\mathrm{p}<0.001)$. These changes plateaued by six months. A change in construct height (mean $3.4 \mathrm{~mm}$ ) occurred by six months $(\mathrm{p}<0.05)$.

Conclusions: The Codman anterior cervical plate provides effective fixation with load sharing and is effective in achieving fusion with a 94\% success rate on independent blinded evaluation. Direct comparison with rigidly locked devices is required to definitively establish the optimum method for anterior cervical fixation.

\section{G-02}

Comparison of primary and secondary spinal epidural abscesses

S.H. Khan, M.S. Hussein, S. Hattingh, R.W. Griebel (Saskatoon, Saskatchewan)

Background: Spinal epidural abscess (SEA), a rare disorder, may cause devastating sequelae. Two groups of SEAare defined: SEA alone (primary/group I), SEA with osteomyelitis (secondary/group II). Both groups were compared.

Methods: Retrospective analysis was performed of all SEA between 1980-2000. Cases were identified by radiological and/or operative findings. We assessed survival, age, infecting organism, duration of hospitalization, management and outcome. Data was analyzed using ANOVA.

Results: Of 29 cases identified, 8 (27\%) were primary and 21 (72.4\%) secondary. Mean age was 55.7 and 52.15 in groups I and II. Mean duration of hospitalization was 46.5 in the former and 35.81 days in the latter group $(\mathrm{p}=0.38)$. Correct admitting diagnosis (epidural abscess) was made in $62.5 \%$ and $23.81 \%$ of 
primary and secondary groups $(\mathrm{p}=.05)$. Entire group I and $52.38 \%$ of group II underwent surgery $(\mathrm{p}=0.01) .37 .5 \%$ vs $23.8 \%$ had good outcome; $12.5 \%$ vs $19.04 \%$ had fair outcome; $37.5 \%$ vs $19.04 \%$ had poor outcome in groups I and II. S. aureus was present in $75 \%$ group I and $57 \%$ group II. Mortality was $12.5 \%$ and $14.28 \%$.

Conclusion: SEA in both groups has similar characteristics.

\section{G-03}

\section{A prospective trial of outpatient lumbar microdiscectomy in 100 cases}

\section{A. Singhal, M. Bernstein (Toronto, Ontario)}

Background: Outpatient surgery saves patients the risk of nosocomial complications and saves health care dollars. Patients undergoing lumbar microsurgical discectomy are excellent candidates for outpatient surgery.

Methods: From February 1997 to November 2000, one hundred consecutive patients of the senior author were selected for outpatient lumbar microdiscectomy (OLM). Patients were excluded if they had significant co-morbidities, lived a significant distance out-of-town, or if they were admitted from the emergency department (ie. cauda equina syndrome and/or requiring IV/IM analgesia). Success was defined as discharge home from the day-surgery unit about 4 hours after surgery with no admission to hospital prior to follow-up.

Results: 128 lumbar microdiscectomies were performed during the study period. 26 patients were excluded based on the above criteria, and two patients requested not to participate in the study. 94 of 100 successfully completed the protocol. Six patients were admitted from the day surgery unit; two dural tears and 4 anaesthetic side-effects. No patient was readmitted to hospital after discharge and no complications of early discharge were observed.

Conclusions: Lumbar microdiscectomy can be performed safely as an outpatient procedure with high patient satisfaction. With increasing pressure on the hospital system, this may become a viable option in neurosurgical units worldwide.

\section{G-04}

\section{Synovial cysts as a cause of lumbar radicular compression}

\section{Abhay Varma, William Tucker, Paul Muller (Toronto, Ontario)}

Background: The differential diagnosis of acute spontaneous paraparesis, cauda equina claudication and/or lumbar radiculopathy is substantial. Disc rupture or sequestration, spondylogenic encroachment on the nervous elements, neuropathic pathology such as tumor, extradural abscess or hematoma and vascular malformations are considered. Viscerogenic and psychogenic causes need also be considered.

Method: In the interval between June 1991 and March 1999, 549 lumbar laminectomy decompressions were undertaken at St. Michael's Hospital; among these patients, four were identified as having lumbar compression as the consequence of a synovial cyst. These patients presented with the full range of clinical syndromes.
Results: There were two males and two females; their ages were 45, 72, 72 and 73, respectively. Two presented with typical cauda equina claudication as the consequence of spinal stenosis caused by the synovial cyst. One presented with an acute posttraumatic sciatic radicular syndrome. One presented with acute paraparesis as the consequence of hemorrhagic expansion of the synovial cyst. All were markedly improved by surgical decompression. In each case the CT or MRI revealed a globular mass lesion in the dorsolateral portion of the spinal canal, associated anatomically with the facet joint. In each case, the cyst caused dural compression.

Conclusion: Lumbar synovial cysts may present with the full range of lumbar syndromes and respond well to surgical extirpation.

\section{EPILEPSY SURGERY}

\section{H-01}

\section{Bilateral temporal lobe epilepsy: selecting surgical candidates using depth electrodes and SEEG}

Matt Wheatley, W. Boling, A. De Almeida, N. Bernasconi, A. Bernasconi, A. Olivier (Montreal, Quebec)

Background: Individuals with temporal lobe epilepsy constitute a diverse group of patients with a wide range of surgically remedial problems. Among the most difficult of these problems are patients with presumptive bitemporal epilepsy. Magnetic resonance imaging (MRI) and hippocampal volume measurements have helped select appropriate surgical candidates. However, depth electrode placement and stereoelectroencephalography (SEEG) remains an important adjunct in selecting surgical candidates. Since 1982, 110 patients with presumptive bitemporal epilepsy have had depth electrodes implanted to help lateralize or localize the epileptic focus. Cendes et al. reported on 21 patients with SEEG and MRI hippocampal volumetric measurements (17 of whom had surgery) (Brain 119:1317-1326). We have continued to follow these patients and accrue new patients.

Methods: We have retrospectively reviewed 60 patients with presumptive bitemporal epilepsy who have had both SEEG and MRI hippocampal volume measurements. In an attempt to predict which patients are likely to have a good surgical outcome, sensitivity, specificity, and the positive and negative predictive value have been calculated for all preoperative tests.

Results: Average follow-up for the surgical patients has been greater than three years. $58 \%$ of the surgical patients have had a good outcome (Engel Class I or II), including $45 \%$ who are seizure free. Only the MRI hippocampal volume measurements $(\mathrm{p}<.02)$ and depth electrode ictal onsets $(\mathrm{p}<.01)$ proved statistically significant. The sensitivity, specificity, positive and negative predictive values for these two tests were $60 \%$, $76 \%, .66, .70$; and $70 \%, 71 \%, .66$ and .75 respectively.

Conclusion: Seizure etiology, scalp electroencephalography, age at seizure onset and duration of epilepsy were not helpful in predicting which patients would benefit from surgery. Despite many recent technical advances such as volumetric imaging, depth electrode recording of ictal seizure onsets remains an 
important tool in selecting surgical candidates for temporal resection.

\section{H-02}

\section{Multiple subpial transections in the surgical management of pediatric epilepsy}

James T. Rutka, Jeffrey Blount, Walter Hader, William Langburt, Hiroshi Otsubo, O.Carter Snead (Toronto, Ontario)

Background: Multiple subpial transection (MST) is a technique that is being increasingly used in the surgical management of epilepsy. It allows the interruption of intracortical horizontally oriented fibers and spares the vertically or radially oriented fibers. As such, it impairs the generation of epileptiform spikes but preserves the tissue's capability for normal neurologic function. As such, it allows treatment of epileptiform activity in eloquent cortex. There are currently no published reports of the use of this technique in pediatric patients.

Methods: Between 1996 and 2000, 32 pediatric patients (age 18 or less) (20 female, 12 male) underwent multiple subpial transections at The Hospital for Sick Children. At least six months follow-up is available for all.

Results: Preoperative seizure patterns and frequencies varied widely. All were refractory to medical management. All underwent placement of subdural grid array and mapping of epileptogenic foci. Twenty seven patients underwent simultaneous frontal or temporal tissue removal (Group 1) and 5 underwent MST alone (Group 2). Hemiparesis (new or worse) was transiently noted in nine patients and dysphasia in six patients. One patient demonstrated psychiatric deterioration four months following surgery. For patients in group 1, 24/27 are Engel class I or II. For patients in group 2, 3/5 are Engel class I or II.

Conclusions: These preliminary observations suggest that multiple subpial transections represent a useful, safe and effective tool in the surgical treatment of epileptogenic foci in eloquent cortex in pediatric patients.

\section{H-03}

Selective amygdalo-hippocampectomy in temporal lobe epilepsy: results in 200 patients

André Olivier, F. Andermann, F. Dubeau, A. Abosch, N. Poulin, W. Boling (Montreal, Quebec)

Background: Numerous studies of the electrophysiology and neuropathology of temporal lobe epilepsy have demonstrated the mesial temporal structures to be the site of seizure origin in the majority of cases. This is the rationale for a selective amygdalohippocampectomy approach first introduced by Niemeyer.

Method: A series of 200 consecutive patients operated for mesial temporal epilepsy diagnosed by clinical features, the presence of mesial inter ictal and ictal discharges on EEG and the demonstration of hippocampal sclerosis and atrophy on MRI. The image guided technique developed at the MNI uses a transcortical approach and consists in a strictly endopial resection of the hippocampal formation and amygdala.
Results: Ninety percent of patients who underwent selective amygdalo-hippocampectomy at the MNI realized a cessation of seizures or greater than $90 \%$ reduction. Poor results are seen in instances of neocortical, multifocal and bitemporal epilepsy. Neurological complications consist in one case of transient 3rd nerve paresis and three cases of transient dysphasia. Results are excellent on seizure tendency with minimal risks.

Conclusion: Selective amygdalo-hippocampectomy is, in our experience, the procedure of choice for mesial temporal epilepsy in well-selected patients.

\section{CHILD NEUROLOGY}

\section{I-01}

\section{Mortality in childhood onset epilepsy is increased but associated with etiology - a proof of what we always thought}

\section{C.S. Camfield, P.R. Camfield, P. Veugelers (Halifax, Nova} Scotia)

Background: Possible death in childhood epilepsy worries families. We documented incidence and causes of death in a population-based cohort over 15-25 years follow-up.

Methods: Our Nova Scotia cohort includes all children who developed epilepsy between 1977-85 ( $n=693)$. In 1999, we matched names and birth dates with Provincial Death and Marriage registries. Survivors were documented alive by date of last physician visit from the provincial health care database, supplemented by clinical information.

Results: Overall 25/693 (3.6\%) died. Relative to Nova Scotia youth, mortality was 5.3 times higher in the 1980 s and 8.8 in the 1990 s (standard mortality ratios). Kaplan-Meyer curves showed $4.1 \%$ mortality 15 years after onset compared with $0.6 \%$ (population rate). Causes of death were aspiration (14), sepsis (3), shunt (1), embolism (1), CHF (1), GER+FTT (1), status (1), suicide (1), homicide (1), SUDEP(1). Death rates were: absence epilepsies 0/97, partial and primary generalized epilepsies $12 / 511(2 \%)$, secondary generalized epilepsies onset $>1$ year of age 4/49 (8\%), secondary generalized epilepsies with onset $<1$ year $9 / 36(25 \%)(\mathrm{p}<0.001)$.

Conclusions: Death following the onset of childhood epilepsy is uncommon but higher than the general population. Most children dying have diffuse brain disease with secondary generalized epilepsy. SUDEP is very rare.

\section{I-02}

\section{Surgical treatment of epilepsy in children with cortical dysplasia: a review of 42 cases}

W.J. Hader, J.T. Rutka, D. Vanderaa, H. Otsubo, O.C. Snead III, A. Tyagi, A. Ochi, S. Chitoku (Toronto, Ontario)

Background: Aggressive early surgical management of medically refractory epilepsy in children, in addition to improving seizure frequency, may result in improvements in cognition, memory and behaviour. We present the surgical results of a large series of pediatric patients with intractable 
epilepsy and a single pathology, cortical dysplasia, at The Hospital for Sick Children.

Methods: A retrospective review of pediatric patients, undergoing surgical treatment of medically refractory epilepsy secondary to cortical dysplasia was performed from April, 1989 to February, 2000. Patients were thoroughly investigated with preoperative EEG, EMU, MRI, fMRI, PET scanning, MEG and neuropsychological evaluations. Intraoperative electrocorticography or subdural grid monitoring was performed in all patients. Intraoperative image guidance with the ISG want were used to selected cases. Seizure outcome with classification according to Engel.

Results: A total of 42 children were included in the study. Patients had epilepsy for a mean of 5.3 years prior to surgical intervention and the average age at treatment was 7.1 (2 mos - 18 years) years old. Thirty patients'surgery involved extratemporal resections. Nineteen patients had greater than two years of follow-up of which nine $(47 \%)$ patients were seizure-free and seven patients had Engel Class II or III outcome. Improvements in development were seen in 28 of 32 (93\%) of patients with mild or severe delay as determined by preoperative neuropsychological assessment. Verbal memory in two, new dysphasia in one patient, and wound infection in six patients.

Conclusion: Surgical treatment of intractable epilepsy in young children with cortical dysplasia resulted in $47 \%$ of patients being seizure-free at two years follow-up and greater than $90 \%$ reduction in seizure (Engel Class I-III) in $94 \%$ of children. These data suggest that early treatment of epilepsy in children with cortical dysplasia will have a long-lasting favourable impact on their lives.

\section{I-03}

\section{Pre-surgical factors that influence seizure outcome in children after temporal lobectomy}

\section{N.C. Sin, H. Otsubo, O.C. Snead, J. Rutka, S. Chuang, E. Kerr, M.L. Smith, $\underline{\text { S. Weiss (Toronto, Ontario) }}$}

Background: Temporal lobe epilepsy is a common epilepsy syndrome and a common cause of medical intractable epilepsy. A large series of children who underwent temporal lobectomy for intractable epilepsy were reviewed to evaluate predictors of seizure-free outcome.

Methods: Retrospective analysis was done of children ages 118 years who had anterior temporal lobe resection between 1995 and 1999 at the Hospital for Sick Children. Seizure outcome was graded according to Engel Classification. All variables were evaluated statistically using univariate tests for their relationship to seizure outcome.

Results: 29 children (15 male) were included. Seizure outcome obtained at follow-up from 12-67 months (mean=43 months) was: class I $(n=20)$, class II $(n=4)$, class III $(n=4)$, class IV $(n=1)$. Absence of mental retardation $(\mathrm{p}<0.012)$ predicted seizure-free outcome. Seizure-free outcome was $71 \%$ vs. $50 \%$ for children with concordance of interictal, ictal, electrophysiologic data and neuroimaging vs. those with bilateral interictal or neuroimaging findings. Trends to better outcome were shown with presence of a structural lesion.

Conclusions: In this case series, pre-operative normal intelligence predicted seizure-free outcome. Concordance of preoperative data and presence of a structural lesion are additional important factors to consider in the pre-surgical evaluation of children with temporal lobe epilepsy.

\section{I-04}

\section{Non-febrile generalized status epilepticus is always focal in origin}

M.M.S. Jan (Jeddah, Saudi Arabia), B.G.R. Neville, R.C. Scott, T.C. Cox (London, UK)

Background: Non-febrile generalized status epilepticus (SE) is a common neurological emergency. Our objectives were to study children with recurrent generalized SE and assess the evidence for focal origin.

Methods: A cohort of children with recurrent generalized SE and intractable epilepsy were identified at the medical unit of St Piers, Lingfield, UK. This is a school, offering special education and medical services for children with severe epilepsy. The school is linked to the Institute of Child Health and Great Ormond Street Hospital in London. Focal clinical, radiological, or electrographic features were identified by chart review. One blinded neuroradiologist reviewed all available CT and MRI films.

Results: 19 children were included with ages ranging between 6-22 years (mean 15.5, SD 4). There were $13(68 \%)$ males and six $(32 \%)$ females. Most children (89\%) had severe cognitive impairment and behavioral problems. All children had multiple seizure types and $95 \%$ had episodes of simple or complex partial seizures. Twelve $(63 \%)$ had SE with focal clinical features at onset, ictus, or offset. Focal brain abnormalities were detected on $25 \%$ and $58 \%$ of CT and MRI films respectively. Most children (95\%) had focal and $29 \%$ had multifocal epileptiform discharges on EEG. Overall, 18 children had focal features on at least one EEG. Of 11 (58\%) children with ictal EEGs, 91\% had focal features at the seizure onset.

Conclusions: The majority of children with apparently generalized SE have focal clinical, radiological, or electrographic features. This supports a focal origin of SE in most children with intractable epilepsy.

\section{I-05}

\section{The effect of age on hypoxic-ischemic brain damage - the role of cerebral energy metabolism}

\section{J.Y. Yager, E.A. Armstrong, J. Asselin, J. Thornhill (Saskatoon, Saskatchewan)}

Background: Our laboratory has developed a model of hypoxic-ischemic (HI) brain damage in rats aged 10 to 180 days. Rats receive an identical ischemic insult for 35 minutes. In contrast to previously held dogma, the immature brain is actually more sensitive to the damaging effects of an $\mathrm{HI}$ insult than is the juvenile or adult brain (Dev. Brain Res. 1996, 93: 143-154).

Objective: To determine the role cerebral energy metabolism has on HI brain damage in age groups ranging from birth to adulthood.

Methods: Rats aged 10, 21, 63, and 180 days underwent 
LE JOURNAL CANADIEN DES SCIENCES NEUROLOGIQUES

halothane anesthesia, unilateral common carotid artery ligation, and exposure to $12 \%$ oxygen for 35 minutes. At 0,15 , and 35 (terminus) minutes $\mathrm{HI}$, and at 60 minutes of recovery in room air, the brains were funnel-frozen in liquid nitrogen, and subsequently maintained at $-70^{\circ} \mathrm{C}$. Portions of the cerebral cortex in the distribution of the middle cerebral artery, and the hippocampus, of the hemisphere ipsilateral to the ligation were analyzed for concentrations of high-energy phosphate compounds [phosphocreatine (PCr), ATP,ADP, AMP] as well as brain glucose, lactate, pyruvate, beta-hydroxybutyrate, acetocetate, and alpha-ketoglutarate.

Results: No differences were found between the hippocampus and cortex. During HI, brain concentrations of lactate increased significantly above their homologous controls in all age groups $(\mathrm{p}<0.05)$. Concentrations of high-energy phosphate compounds (ATP and PCr) decreased to $<20 \%$ of control only in the 10 and 21 day animals $(\mathrm{p}<0.01)$, whereas in the other age groups, no significant alterations from control were detected.

Control: 1) Despite receiving an identical cerebral insult, high-energy phosphate compounds were depleted in only the most immature rats (10 and 21 days). 2) A significant reduction of high-energy phosphate compounds is required before brain damage will occur, and 3) The juvenile and adult brain is more resistant to energy depletion during an equivalent $\mathrm{HI}$ insult compared to the immature brain.

\section{I-06}

\section{Long-term outcome of children with Guillain-Barré syndrome pre and post the introduction of intravenous gamma globulin}

\section{Darcy Fehlings, J. Vajsar, D. Stephens (Toronto, Ontario)}

Background: This study compares the long-term muscle recovery of children with GBS treated after the introduction of IVIG (1988), to historical controls.

Methods: Cases are children with GBS, at least two years postrecovery, admitted to HSC between 1988 to 1995. Manual muscle strength testing was done on 35 muscle groups, graded between 0 to 10 points on the modified Medical Research Council scale. A literature review identified one study evaluating the long-term sequelae of children with GBS prior to the use of IVIG, representing a historical control group. (J Pediatr 1975; 86: 356-359)

Results: Within the cases, forty-seven subjects with GBS were identified, of which 30 received IVIG. Thirty-one subjects $(66 \%)$ agreed to participate. Twenty-three per cent had muscle weakness with at least one muscle group at a grade 6 level. The neck flexors $(\mathrm{x}=8.65$, range 6 to 10$)$, and hip abductors $(\mathrm{x}=$ 8.84 , range 5 to 10 ) were the weakest muscles. All children were ambulatory and none wore braces. In the historical control group, 11 of $47(23 \%)$ children had long-term sequelae with "many" requiring short leg braces.

Conclusions: Despite the introduction of IVIG, 23\% of children with GBS had long-term muscle weakness. IVIG speeds the rate of muscle recovery but does not decrease the percentage of children who develop long-term GBS sequelae. However, the persisting muscle weakness is milder, with no child in the case group requiring braces.
I-07

Presumed pre- or perinatal arterial ischemic stroke: risk factors and outcomes

Meredith Golomb (Toronto, Ontario)

Winner of The President's Prize (See page 8)

\section{GENERAL NEUROLOGY}

$\mathbf{J}-01$

Factors associated with early and late unemployment in multiple sclerosis patients

K. Busche, L. Metz (Calgary, Alberta), J. Fisk (Halifax, Nova Scotia)

Winner of the Frances McNaughton Memorial Prize (See page 8 )

$\mathbf{J - 0 2}$

Seizure outcome and health-related quality of life in surgical versus medical management of epilepsy: an 8 year prospective outcome study

R.S. McLachlan, P.A. Derry, M. Morris (London, Ontario)

Background: We report a prospective 8.5 year follow-up of a cohort treated by medical or surgical (temporal lobectomy) management in epilepsy $(\mathrm{N}=39)$, measuring seizure outcome and quality of life (QOL).

Methods: There were two groups: surgery $(\mathrm{n}=29)$ and Medical Management $(\mathrm{n}=10)$, and three assessment periods (baseline, two years, 8.5 years). Medical management control patients had inpatient evaluation as surgical candidates but did not undergo surgery. Measures: seizure frequency and QOL (QOLIE-89).

Results: At follow-up, $73 \%$ of operated patients were seizurefree, compared to $22 \%$ of medically-managed patients $(\mathrm{p}<.001)$. Greater than $90 \%$ seizure improvement was seen in $10 \%$ of operated patients and in $33 \%$ of medical patients. Less than $90 \%$ seizure improvement was obtained in $17 \%$ of operated and $44 \%$ of non-operated patients. Seizure-free patients showed improvement $(\mathrm{p}<.05)$ in 13 of 14 QOLIE-89 domains, compared to baseline; not-seizure-free patients showed improvement in only two of 14 domains.

Conclusions: This study is unique for its length of follow-up, prospective design, and measurement of both seizure outcome and QOL. Temporal lobectomy appears superior to medical management for long-term seizure reduction, as well as for sustained improvement in QOL. Improved QOL seen among surgical patients at two years was maintained at 8.5 years. 
$\mathbf{J - 0 3}$

In myasthenia gravis, patients whose thymus secretes antibodies, have shorter time to pharmacological remission after thymectomy

\section{Hans Katzberg, Tariq Aziz, Joël Oger (Vancouver, British Columbia)}

Background: Myasthenia gravis (MG) is a neuromuscular disease in which acetylcholine receptor antibodies (AchRAb) are elevated and for which, due to the high proportion of thymic pathology, thymectomy is often used as a treatment despite illdefined indications.

Methods: Our study compared improvement postthymectomy in MG patients with different immunologic activity. $42 \mathrm{MG}$ patients were evaluated pre-thymectomy and at 3,6,12, 24 and 60 months postoperatively for clinical signs and symptoms, quantitative serum AchRAb levels and medication dosages (as reflection of therapeutic needs). AchRAb secretion in vitro from thymocytes obtained at surgery was determined and used to segregate patients into secretors and non-secretors.

Results: Secretors did better clinically with $80 \%$ decrease in mestinon requirements at 24 months; furthermore, it took three months to start reducing prednisone dose. Non-secretors had $15 \%$ decrease in mestinon requirement at two years and prednisone took six months to decrease. Postoperative serum AchRAb, while remaining low at $<10 \mathrm{nmol} / \mathrm{L}$ in non-secretors, rose progressively in secretors to $64 \%$ above baseline in the first six months before dropping to $<10 \mathrm{nmol} / \mathrm{L}$ at 24 months.

Conclusions: We conclude that patients who secrete AchRAb in culture have shorter time to pharmacological remission than non-secretors, despite increasing serum AchRAb levels in the first 12 months postoperatively.

\section{J-04}

\section{Bitemporal EEG abnormalities may favorably predict response to vagal nerve stimulation in patients with medically refractory epilepsy}

\section{D.B. Clarke, R.M. Sadler, S. Rahey (Halifax, Nova Scotia)}

Background: Vagal nerve stimulation (VNS) has become an accepted treatment option for those patients with medically refractory epilepsy and for whom no traditional resective surgery can be offered. Studies thus far have failed to identify specific patient subtypes that would predict a favorable outcome. The purpose of this study is to examine our experience to determine whether location of epileptiform activity can predict a favorable response to VNS.

Methods: We have treated 14 patients with VNS and mean follow-up is one year (range: 3-18 months). Epilepsy has been of various etiologies and all but two patients had more than one seizure type. EEG localization of epileptiform activity varied, consisting of hemispheric (2), fronto-temporal (1), parietal (1), bioccipital (1), multifocal (3) and bitemporal (6) foci. Six patients had 12 previous resective surgeries. We have examined the results of VNS implantation in terms of reduction in seizure frequency.

Results: Consistent with previous studies, we have observed a significant response (as defined by $>50 \%$ reduction in seizure frequency) in approximately one-third of patients. All patients showing a significant response have had bitemporal EEG abnormalities.

Conclusion: Our early experience in treating patients with severe medically refractory epilepsy by VNS indicates that patients with bitemporal foci may have a more favorable outcome in terms of seizure reduction.

\section{$\mathbf{J}-\mathbf{0 5}$}

\section{Form perception distortion after cerebral hemispheric lesions}

James A. Sharpe, Ji Soo Kim, Josee Rivest (Toronto, Ontario), James Intrilligator (Boston, MA, USA)

Purpose: When two shapes are presented successively and briefly to normal subjects, the form of the second shape (test) appears dissimilar to the form of the first shape (priming). This shape distortion effect may originate from adaptation of nonretinotopic and non-feature-specific neurons in higher visual areas of the ventral stream of visual processing. To determine effects of cerebral lesions on this shape distortion, we measured it in 15 patients with cerebral hemispheric lesion and in normal subjects.

Methods: For each trial, flashed in succession were a priming rectangle (vertically or horizontally; $45 \mathrm{~ms}$ ), a gap of stimulus onset asynchrony $(180 \mathrm{~ms})$, a test circle $(60 \mathrm{~ms})$, and a randomdot mask $(255 \mathrm{~ms})$. The priming rectangle and test circle were presented in the middle of each quadrant of intact field. In intermixed control trials, there was no priming rectangle and the test shape was a circle or an ellipse. After each trial, using the method of adjustment, the \% elongation [(longer diameter) (shorter diameter)]/ (shorter diameter) X 100 of the reproduced ellipse was computed. The extent of lesion in brain MRI of each patient with abnormal shape distortion effect was mapped on templates of axial brain anatomy.

Results: Seven patients showed absence of the normal shape distortion effect. Three of them also had impaired perception of intermixed control shapes. In six out of 7 patients with no distortion effect, brain lesions were overlapped in the posterior part of the middle and inferior temporal gyri. The cortical area included in the overlap encompassed Brodmann area 37, the posterior aspect of area 21 , and the ventrocaudal portion of area 39. Seven patients with lesions in other areas showed a distortion comparable to that of controls (average, $12 \%$ elongation).

Conclusions: Loss of the shape distortion effect occurs in patients with damage to the posterior part of middle and inferior temporal lobe. The results provide evidence that the shape distortion effect depends on the coding of cells in the ventral stream of processing. The posterior and inferior temporal lobe are critical for perception of this shape distortion. 
J-06

\section{The patient with a sagging brain}

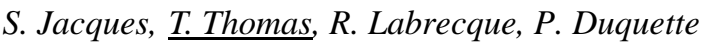 \\ (Montreal, Quebec)}

Background: Often patients with intracranial hypotension present with clinically mild symptoms that remit spontaneously. Few cases with major neurological dysfunction have been reported.

Methods: We present the case of a 60-year-old woman who was diagnosed with intracranial hypotension leading to a severe encephalopathy in three months.

Results: The patient initially complained of a new persisting headache. She then developed memory disturbances, apathy, drowsiness, discrete cerebellar and left pyramidal tract dysfunction. The brain CT revealed bilateral subdural effusions. They were drained and the liquid was compatible with subdural hematomas. Collections reappeared and the patient developed stupor and bilateral Babinski signs. Drainage was repeated. The surgeons noted a sagging brain and had to aspirate in order to retrieve liquid. Brain biopsy revealed a dense lymphocytic dural infiltrate overlying normal brain parenchyma. Serial brain MRI showed progressive descent of midline structures and a Chiari I malformation prompting the diagnosis of intracranial hypotension. The patient underwent a complete spontaneous recovery.

Conclusions: Intracranial hypotension can present dramatically and lead to invasive investigation. Headache, spontaneous subdural effusions and descent of midline cerebral structures on imagery are indices of intracranial hypotension and should orient investigation and treatment.

\section{$\mathbf{J - 0 7}$}

\section{Validation of a weight-based nomogram for the use of heparin in transient ischemic attack or stroke (HANST study)}

\section{Toth, C. Voll (Saskatoon, Saskatchewan)}

Objective: To validate the use of a weight-based nomogram for heparin adjusted therapy during hospital admission of patients with transient ischemic attack (TIA) or stroke.

Background: Intravenous heparin therapy is often used in patients presenting with TIAor stroke as either bridging therapy for anticoagulation with coumadin, or as primary therapy in suspected intracranial arterial dissection, crescendo TIAs, or suspected hypercoagulable states.

Methods: A prospective, single-blinded, randomized clinical trial was undertaken to compare the use of a specially-designed weight-based nomogram (HANST) against the traditional method of physician ordered heparin therapy for patients admitted with TIA or stroke. The trial was not designed to examine the efficacy of heparin therapy, but to examine the use of the nomogram for labour requirements, costs of monitoring, safety, length of heparin therapy, and user-friendliness.

Results: A total of 206 patients were enrolled and randomized. Each group of patients were comparable. HANST patients had a significantly lower initial partial thromboplastin time (PTT) than non-nomogram patients (Mann-Whitley, $\mathrm{p}<0.05$ ). HANST patients had a significantly greater probability of achieving therapeutic anticoagulation levels earlier than nonnomogram patients (t-test, $\mathrm{p}<0.01$ ). HANST patients also had fewer supratherapeutic coagulation results (Mann-Whitley, $\mathrm{p}<0.05$ ), fewer correction errors (Mann-Whitley, $\mathrm{p}<0.05$ ), and fewer calls to housestaff regarding anticoagulation (MannWhitley, $\mathrm{p}<0.001)$. The number of complications, time to discontinue heparin, and time to discharge patients was not significantly different. Survey of housestaff and nursing staff showed preference for nomogram use.

Conclusion: Use of the HANST nomogram is a user-friendly method of maintaining heparin infusions, and is associated with a lower initial PTT, shorter time to achieve therapeutic anticoagulation range, fewer errors in dosage adjustment, and decreases labour on part of housestaff and nursing staff.

\section{NEURO-ONCOLOGY / NEUROSCIENCE}

\section{K-01}

\section{Transplanted postmitotic human neurons survive and mature in an experimental rat spinal cord injury model}

\section{S.D. Christie, I. Mendez (Halifax, Nova Scotia)}

Background: Novel treatment strategies for spinal cord injury, including neural transplantation, are under investigation. We report on the use of a human cell line which, following treatment with retinoic acid, is restricted to a neuronal lineage (hNT). These cells have been used for transplantation in animal models of neurologic disease such as Parkinson's, Huntington's and stroke. A phase 1 study of hNT grafts in stroke has recently been reported in humans. The aim of this project was to assess the capacity of transplanted hNT cells to survive and mature in a spinal cord injury model.

Methods: Six female Wistar rats received a left hemisection at T10. Three days later, the animals were transplanted with a cell suspension of hNT neurons. Animals were immunosuppressed with cyclosporin A. Twelve weeks later, the animals were perfused and the tissue processed for human neuron specific enolase and human neural cell adhesion molecule immunohistochemistry.

Results: Surviving grafts were seen in four of the six rats. Axonal projections were identified extending both rostral and caudal from the region of the lesion, essentially bridging the hemisection. Positive staining was measured up to $9 \mathrm{~mm}$ distal to the graft site.

Conclusions: Transplanted hNT cells can survive and mature in the hostile environment following spinal cord injury. Evidence that these grafts can project axons across the region of spinal injury could prove relevant for future treatment strategies. 


\section{K-02}

\section{Expression of notch-1 protects U251 astrocytoma cells from apoptosis in response to serum depletion}

S. Lee, T.G. Mainprize, M.D. Taylor, Y. Akiyama, M. McGill, K. Akaishi, J. McGlade, J.T. Rutka (Toronto, Ontario)

Background: The highly evolutionarily conserved notch signaling pathway is activated by ligand binding, such as jagged1 , to the transmembrane receptor notch. This pathway is known to regulate a multitude of cellular processes including differentiation, proliferation and cell death.

Methods: Using the tet-off gene regulatory expression system, we transfected full length notch-1 cDNAinto the notchnegative glioma cell line U251. We examined the role of notch1 expression in U251 cells that were serum starved. Protein expression levels of various regulators of apoptosis were examined by western blot analysis

Results: Parental U251 (U251 ${ }^{\mathrm{P}}$ ) and transfected but nonnotch-1 expressing cell lines (U251 ${ }^{\mathrm{N}-}$ ) underwent apoptosis following serum depletion, however, the notch-1 expressing $\mathrm{U} 251$ cells $\left(\mathrm{U} 251^{\mathrm{N}+}\right)$ demonstrated resistance to the apoptotic signal. There was no significant difference in Bax protein expression in the different clones. Interestingly, the expression of Akt/PKB was upregulated in $\mathrm{U} 251^{\mathrm{N}+}$ cells. Following serum starvation, phosphorylated Akt/PKB was down-regulated to undetectable levels in $\mathrm{U} 251^{\mathrm{P}}$ and $\mathrm{U} 251^{\mathrm{N}-}$, whereas $\mathrm{U} 251^{\mathrm{N}+}$ maintained constant expression levels of Akt/PKB.

Conclusions: These data suggest that notch-1 receptor activation protects U251 glioblastoma cells from undergoing apoptosis following serum starvation possibly through an Akt/PKB-dependent cell survival pathway.

\section{K-03}

\section{Truncating mutations of Human Suppressor of Fused} $(h S u(f u))$ in pediatric medulloblastomas: implications for Sonic Hedgehog and Wnt signaling

Michael D. Taylor, David Hogg, Corey Raffel, Todd G. Mainprize, C.C. Hui, Ling Liu, X. Zhang, Sharon Chiappa, Steve Scherer, W. Dura, James T. Rutka (Toronto, Ontario)

Background: Up to $30 \%$ of medulloblastomas show loss of heterozygosity (LOH) on chromosome 10q. We evaluated candidate tumor suppressor genes at that locus including PTEN, $\beta$-TRCP, and Suppressor of Fused ( $h \mathrm{Su}(f u))$ in cases of sporadic medulloblastoma. Patients with Gorlin syndrome have overactive Sonic Hedgehog signaling and develop medulloblastoma. Humans with germline APC mutations have a high risk of developing medulloblastoma (Turcot syndrome) due to excessive Wnt signaling.

Methods: We performed mutational analyses and functional characterization of identified mutants of $h S u(f u)$.

Results: We did not observe any mutations in PTEN or $\beta$ $T R C P$, but found mutations of $h S u(f u)$ in 3/36 medulloblastomas. All three medulloblastomas with $h S u(f u)$ mutations showed LOH, suggesting that $h S u(f u)$ acts as a classical Knudsen type tumour suppressor gene. Wild-type $h S u(f u)$ can bind the proto-oncogene hGli1, but a medulloblastoma derived mutant, $h S u$ (fu)- $\Delta$ ex 8 cannot. hGli1 is exported from the nucleus by $h S u(f u)$-wild type but not by $h S u(f u)-\Delta e x 8 . h S u(f u)_{\text {-wt }}$ binds $\beta$-catenin and exports it from the nucleus whereas $h S u(f u)-\Delta e x 8$ can bind $\beta$-catenin but cannot export it from the nucleus.

Results: These results suggest that $h S u(f u)$ acts as a classical tumor suppressor gene in a subset of medulloblastomas. Loss of function of $h S u(f u)$ predisposes to over-activation of Sonic Hedgehog and Wnt signaling, two pathways known to be important in medulloblastoma.

\section{K-04}

Establishing the involvement of the Tie2 pathway in pathological angiogenesis of astrocytomas

\section{G. Zadeh, L. Roncari, A. Guha (Toronto, Ontario),}

\section{Kontos (Durham, North Carolina, USA)}

Background: Growth of astrocytomas is dependent on tumor angiogenesis. Tie2 is a novel endothelium-specific receptor tyrosine kinase, known to play an important role in development of embryonic vasculature. We hypothesize that the Tie2 pathway is involved in pathological development of astrocytoma vessels.

Methods: Two strategies were used to inhibit Tie2 receptor. First, an adenoviral vector was constructed to deliver a recombinant, soluble Tie2 receptor (AdExTek) that blocks Tie2 activation. Second, a soluble form of the extracellular domain of murine Tie2 (ExTek6His) was developed using a baculovirus expression system in insect cells. ExTek6His acts as a dominant negative mutant. AdExTek and similarly ExTek6His protein were directly administered into tumor cores of twenty mice with xenografts of human astrocytoma tumors. Two weeks after injection, tumors were analyzed for rate of growth and vessel development. Functional viability of AdExTek and ExTek6His was confirmed by demonstrating inhibition of tubule formation in in vitro assays.

Results: All tumor specimens demonstrated abundant Tie2 levels on the endothelium. A statistically significant decrease in growth and vascular density of the tumors compared to control was observed $(\mathrm{p}=0.001)$.

Conclusion: Our data suggest that Tie2 pathway is involved in vascular growth of astrocytomas and Tie2 inhibitors may prove to be effective anti-angiogenic agents.

\section{K-05}

Testing the go or grow hypothesis: results from a twodimensional migratory model using medulloblastoma cell lines

\section{A. Corcoran, R.F. Del Maestro (Montreal, Quebec)}

Background: Medulloblastoma remains the most common malignant pediatric intracranial tumour and many patients continue to succumb from recurrent disease. One pattern of recurrence is leptomeningeal growth of tumour as sheets of cells along brain and nerve surfaces. The contribution of mitotic subpopulations of medulloblastoma cells to invasion in twodimensions remains to be defined. The 'grow or go hypothesis' states that cell migration and proliferation are temporally exclusive events, both of which contribute to the progression of 
this disease. We have used a time-lapsed videomicroscopy system to assess the relationship between the migratory and mitotic activities of individual medulloblastoma cells in twodimensional culture.

Methods: Time-lapse videomicroscopy was used to monitor four medulloblastoma cell lines (Daoy, UW 228-1, 2 and 3) in both confluent culture and after partial removal of cells by scraping to monitor directional migration.

Results: Cells in scraped cultures displayed decreased mitotic activity compared to cells in confluent cultures to varying degrees in the different cell lines $(\mathrm{p}<0.001$ to $\mathrm{p}<0.05$, chisquared test). When cell lines were compared in scraped cultures, the cell line with the largest migration distance also displayed the greatest mitotic activity. Subpopulations of nondividing cells and cells dividing once or twice were identified within both scraped and confluent cultures. Although the migration distance was dependent on cell line ( $p<0.001$, ANOVA), within a given cell line the mean distance traveled by individual cells was not dependent on the number of divisions $(\mathrm{p}=0.3)$.

Conclusions: Since decreased mitotic activities were observed when comparing confluent to scraped cultures, cellular microenvironment clearly influences mitotic activity. When medulloblastoma cell lines were compared, a direct relationship between migratory distance and mitotic activity was present among the different cell lines. However, this relationship was not seen when dividing and nondividing cells were assessed within a given cell line. When evaluating the relationship between migratory and mitotic activity of cells, it is appropriate to take into account the models and methodology used.

\section{K-06}

\section{Combination treatment of fructose 1,6-diphosphate and dimethyl sulfoxide for traumatic brain injury in rats}

J.S. Park (Irvine, California, USA), J.C. de la Torre (San Diego, California, USA)

An estimated 695,000 people in North America sustain traumatic brain injuries (TBI) annually for which no effective treatment exists. The present study examined the effect of a drug "cocktail" for acute TBI. Rats (Sprague-Dawley, male, $n=48$ ) were subjected to blunt TBI equivalent to $200 \mathrm{gm} / \mathrm{cm}$ force impact. The injury was induced through a cranial window using a drop weight method. Rats were randomly separated into four groups $(n=12)$ and received i.p. treatments one hour after brain contusion. Sensory-motor assessment was done prior to killing at 19 hours and densitometric neuronal counts made of CA1 at two and 19 hours after TBI. Group DS received the hydroxyl radical scavenger dimethyl sulfoxide, $28 \%$ solution $(250 \mathrm{mg} / \mathrm{Kg})$, group FD was given the glycolytic intermediate fructose 1,6diphosphate, $10 \%$ solution $(135 \mathrm{mg} / \mathrm{Kg})$ and group DS-FD, a combination of dimethyl sulfoxide + fructose 1,6- diphosphate solutions. Control (no TBI) group were given D5W. Except for the control group, osmolar concentration of the treating solutions were the same $(1.7 \mathrm{mOsm})$ for all three groups. Results showed that TBI rats treated with the combination DS-FD showed significantly less neurologic deficits at 19 hours $(\mathrm{p}=<0.01)$ when compared to other groups. Neuronal counts of CA1 showed a higher number of neurons preserved in DS-FD group both at two and 19 hours after TBI $(\mathrm{p}=<.005)$. At 19 hours after brain trauma, improved neuronal protein synthesis in cingulate cortex and significantly less gliosis in dentate gyrus was seen with DS-FD treatment when compared to other treatments. These findings indicate marked neuroprotective action by DS-FD, not seen when either compound was used alone.

\section{K-07}

Role of the metabotropic glutamate receptor mGluR1 in calcium-mediated white matter spinal cord injury

\section{Nicolas Phan, M.G. Fehlings (Toronto, Ontario)}

Introduction: Spinal cord injury (SCI) results in secondary injury mechanisms including glutamate excitotoxicity and calcium imbalance. The mGluR1 glutamate receptor is linked to phospholipase-C activation causing the release of intracellular calcium $\left(\left[\mathrm{Ca}^{2+}\right]_{\mathrm{i}}\right)$ from internal stores via IP3. We have previously shown that mGluR1 blockade improves electrophysiological recovery following SCI.

Hypothesis: We examined the hypothesis that mGluR1 blockade results in attenuated $\left[\mathrm{Ca}^{2+}\right]_{\mathrm{i}}$ rises in SCI.

Methods: Glial cells emitting calcium fluorescence where identified in the white matter of murine spinal cord slices incubated with fluo3. An injury was delivered to the slice with either a) a weight-drop or b) $100 \mu \mathrm{M}$ glutamate for 15 minutes.

Results: Under control conditions, glial calcium fluorescence rose to $183.8 \pm 2.5 \% \quad(n=15)$ after mechanical injury and $179.9 \pm 1.6 \%(n=14)$ following glutamate injury. Blockade of mGluR1 with PHCCC resulted in $\left[\mathrm{Ca}^{2+}\right]_{\mathrm{i}}$ rises of $167.1 \pm 1.8 \%$ following mechanical injury and $134.9 \pm 2.5 \% \quad(n=28)$ after glutamate. Depletion of IP3-sensitive intracellular stores with thapsigargin lead to $\left[\mathrm{Ca}^{2+}\right]_{\mathrm{i}}$ rises of $131.8 \pm 0.5 \% \quad(\mathrm{n}=15)$ following glutamate injury. Slices from mGluR1 knockout mice showed a rise of $145.4 \pm 4.8 \% \quad(n=10)$ following mechanical injury and $119.9 \pm 2.5 \%(n=20)$ following glutamate. Doublelabelling of fluo3-positive cells with GFAP revealed that over $85 \%$ were astrocytes. All results were statistically significant $(\mathrm{p}<0.001)$.

Conclusion: Pharmacological blockade of mGluR1 as well as selective gene deletion of mGluR1 lead to a reduction in the rises of astrocytic $\left[\mathrm{Ca}^{2+}\right]_{\mathrm{i}}$ following SCI. 


\section{Poster Presentations}

\section{CEREBROVASCULAR DISEASE}

\section{P-001}

\section{Multicentre experience of large middle cerebral artery infarction: the role of $\mathrm{CT}$ for predicting fatal outcome}

Philip A. Barber, Andrew M. Demchuk, Jinjin Zhang (Calgary, Alberta), Scott E. Kasner (Philadelphia, USA), Jorg Berrouschot (Berlin), E. Sschmutzhard (Vienna), L. Harms (Bunn), P. Verro, Derk Krieger.

Background: Large middle cerebral artery (MCA) ischemic stroke, when associated with extensive mass effect, can result in brain herniation and death. As yet there are no guidelines to aid the selection of patients for decompression therapies, such as hemicraniectomy.

Methods: This was a multicentre retrospective study of large MCAinfarction requiring neurocritical care. The repeat CTscans performed within 120 hours of stroke onset were assessed for horizontal displacement of septum pellucidum and pineal gland, the size of MCA infarction, involvement of other vascular territories and hydrocephalus. The primary outcome measure was death within 30 days.

Results: A total of 251 patients fulfilled entry criteria into the study of which 201 received conventional medical therapy alone. Bivariate analysis identified the following dichotomised predictors of early death: NIHSS ( $\mathrm{P}<0.03$, OR 2.17 95\% CI 1.124.2), anteroseptal shift ( $\mathrm{P}<0.001$, OR 9.2 95\% CI 4.1-20.63), pineal shift $(\mathrm{P}<0.001$, OR $12.195 \%$ CI 4.74-30.8), ischemia involving additional vascular territories $(\mathrm{P}<0.001$, OR $7.0295 \%$ CI 3.35-14.7), hydrocephalus ( $\mathrm{P}<0.02$, OR $2.1395 \%$ CI $1.15-$ $3.94)$, and temporal lobe involvement ( $\mathrm{P}<0.001$, OR $5.6695 \%$ CI 2.58-12.4). Multivariable analysis was performed but no more complicated relationship was observed between different variables. Anteroseptal shift dichotomised into $<7$ and $\geq 7 \mathrm{~mm}$ had sensitivity $53 \%$, and specificity $93 \%$ for neurological death. Pineal shift dichotomised $<3$ and $\geq 3 \mathrm{~mm}$ had sensitivity $45 \%$ and specificity $94 \%$.

Conclusions: We identified the role of follow-up CT in predicting early death following massive MCA infarction. The dichotomised variables, anteroseptal shift $(\geq 7$ versus $<7 \mathrm{~mm})$ and (pineal shift $\geq 3$ versus $<3 \mathrm{~mm}$ ), if present were strongly predictive of fatal outcome. These follow-up CT parameters require validation before they impact the decision to perform surgical intervention.
P-002

Quebec forum for cardiovascular* health professionals: an initiative of the Heart and Stroke Foundation of Quebec

L. Bertrand (Montreal, Quebec)

In October 1995, a day of reflection brought together more than 125 individuals active in cardiovascular healthcare, under the theme "Heart Health in Quebec: towards the year 2000". The conclusion reached as a result of these reflection mechanisms favoured a concerted approach involving all partners in the cardiovascular healthcare sector. It is within this context that the Quebec Forum for Cardiovascular* Health Professionals was set up.

The Forum wishes to play the role of facilitator to optimise the interventions related to its sector. It also intends to play the role of strategic leader to promote and stimulate interest in cardiovascular health among the population in general and among decision-makers. The wealth of the provincial structure for concerted action reflects the many sectors it brings together: groups involved in healthcare, in promotion/prevention activities, educational activities, research, and the pharmaceutical sector.

Focus groups have been conducted to evaluate the Forum as a whole. All cardiovascular health professionals who participated, perceived the Forum as a credible high quality informative tool, very useful for health professionals.

The Forum's activities: The bulletin Les actualités du coeur and the Forum website, are much appreciated and cyberconferences have been recommended.

* the term cardiovascular includes cerebrovascular

\section{P-003}

Equitable access to acute stroke interventions within a regional acute stroke protocol

D. Howse, C. Bolton, S. Elson, D. Brunet, A. Jackson, M. Melanson, D. Weaver, D. Groll (Kingston, Ontario), R. Riopelle (Montreal, Quebec)

Background: Southeastern Ontario (SEO) has a population of 475,000 and encompasses an area of 20,000 square kilometres. In SEO, Kingston General Hospital (KGH) is the only hospital with 24/7 access to CT, neurology, neuroradiology and neurosurgery. In July 1999, a regional acute stroke protocol (RASP) was implemented to ensure equitable access to evidence-based acute stroke interventions (e.g., rt-PA). As a result, when specified inclusion criteria are met, ambulances bypass the closest hospital and transport patients directly to KGH.

Methods: A 1-year retrospective chart analysis was undertaken for RASP patients (i) to determine if the targeted population was successfully being captured under the RASP, (ii) to 
determine where patients come from geographically, and (iii) to discover what treatments patients received and the outcomes following treatment.

Results: During year 1, there were 403 ischemic strokes in SEO, the RASP was activated 191 times, $40 \%$ of patients bypassed the closest hospital, and $20 \%$ were transferred emergently from a community hospital. Patients from greater than $180 \mathrm{~km}$ away from $\mathrm{KGH}$ benefited from rapid access to diagnostics, intravenous rt-PA, intra-arterial thrombolysis, neurosurgery, and stroke prevention.

Conclusion: The development of a RASP has significantly improved equitable access to acute stroke interventions within a large geographic region in Canada.

\section{P-004}

\section{Bilateral vertebral dissection in a patient with HTLV-1 and Sjogren's syndrome}

\section{K.M. Chapman, J. Oger (Vancouver, British Columbia)}

Background: Human T-lymphotropic virus-1 (HTLV-1) is a human retrovirus which has been identified as the etiologic agent of a slowly progressive myelopathy known as HAM/TSP. HTLV1 infects CD4+ T lymphocytes, causing lymphocytes to become self activated. The spectrum of clinical manifestations known to accompany HTLV-1 includes Sjogren's Syndrome (SS).

Methods: Case report.

Results: A 41-year-old West Coast Native Indian woman with SS experienced sudden spontaneous onset of severe neck pain. A left lateral medullary syndrome was present. Bilateral vertebral artery dissections were apparent on cerebral angiogram. She was found to be HTLV-1 positive (serum and CSF antibody/PCR). In vivo evidence suggests that the Ro (SSA) antigen is found on cerebrovascular endothelium. Immunologically mediated damage to endothelial cells of the vertebral arteries could cause a focal area of weakness. "Spontaneous" dissection often occurs when preexisting vessel pathology exists; in this case a large vessel vasculitis related to SS could create a susceptibility to dissection.

Conclusions: We describe a case of bilateral vertebral dissection in a patient with SS secondary to HTLV-1 infection, a previously unreported association. The role of HTLV-1 in neurology may extend beyond a slowly progressive myelopathy causing spastic paraparesis. Physicians should be aware of the association of HTLV-1 with other immune mediated conditions.

\section{P-005}

\section{Arterial dissections: review of the last decade}

\section{Jean-Francois Chevalier, Donatella Tampieri,} Denis Mélançon, Rolland Brassard (Montreal, Quebec)

Background: Arterial dissections can be responsible for up to $25 \%$ of cerebro-vascular accidents in the young adult population, with a frequent association with vascular trauma. This series aims at describing the risks factor associated in cases encountered in a tertiary care center, with their long-term prognosis. Secondarily, we review the endovascular treatment options for patients not responding to current medical therapy, with presentation of a case treated with a noncovered wall stent.

Method: Retrospective chart review of cases identified in the Radiology department file bank of the Montreal Neurological Hospital.

Results: 32 files were available for review. The mean age at presentation was 43.3 years old. Repartition between sexes was equal, and five patients had the diagnosis of fibro-muscular dysplasia (FMD), one with clinical Marfanoid traits and one having severe arteriosclerotic disease. A clear history of predisposing trauma was present in 12 patients. The majority of these patients were discharged in good neurological condition. One 46-year-old female patient suffering from FMD went on to have an endovascular stent placement seven months after sustaining a right hemispheric stroke. After six months of followup, no recurrence was recorded.

Conclusions: Arterial dissections represent a frequent cause of ischemic stroke in the young adult population, fortunately with a generally good functional prognosis. Nonresponders to current medical therapy can benefit, in well selected cases, from certain endovascular treatments as suggested by the presentation of one case.

\section{P-006}

\section{Epidemiology of cerebrovascular disease of Chinese- Canadians - a 10 year retrospective study}

Joseph Y. Chu, Jason K. Chu, Arthur K. Chung (Toronto, Ontario)

Background: Asians are commonly known to have more intracranial vascular stenosis than Caucasians. They also have a lower incidence of carotid stenosis and various researchers had substantiated this in previously published studies (Brust, 1975; Chu, 1993).

Method: This report is a retrospective 10-year study (19901999) on the epidemiological characteristics of cerebrovascular disease among a cohort of Chinese-Canadian patients seen in a consultative neurological practice in Toronto.

Results: There were 110 Chinese-Canadians compared to 92 Caucasians. For the Chinese-Canadians, the mean age of stroke is 64 y.o. (range 27-89) and the average duration of symptoms is 1.84 years. Stroke type by CT scan shows a statistical significant difference, with Caucasians having more cortical infarcts than Chinese-Canadians ( $50 \%$ vs. $25.5 \%, \mathrm{p}<0.05)$. Only $6.4 \%$ of the Chinese-Canadians compared to $32.6 \%$ of Caucasians had carotid stenosis $(\mathrm{p}<0.05)$.

Conclusions: This study identifies the important characteristics of cerebrovascular disease among ChineseCanadians living in Toronto. Further research in the genetic, ethno-cultural and dietary factors among successive generations of Chinese-Canadians would be vital in understanding how these factors contribute to the various stroke types in this population. 


\section{P-007}

\section{Contralateral carotid dissection after thrombolytic therapy for an acute stroke}

\section{A. Desjardins, F. Evoy (Sherbrooke, Quebec)}

Background: Intravenous thrombolysis is an accepted treatment modality for patients with stroke symptoms of less than three hours duration. Some cases of thrombolysis therapy in patients with arterial dissection have been reported.

Objective: To report a case of carotid dissection three days after thrombolytic therapy for an acute stroke in the opposite carotid territory.

Case presentation: We report the case of a 56-year-old patient who consulted for left carotid artery stroke. Since the patient presented inside the time limit and didn't have any contraindications he received Activase. In the following days the patient improved. However, on the third day post-procedure he developed a right Horner's sign. The cerebral arteriography performed at this time demonstrated the presence of bilateral carotid dissection, worse on the left side. We performed a renal arteriography which demonstrated right renal artery anomalies typical of fibromuscular dysplasia. A right renal artery angioplasty was performed and the patient was anticoagulated with vitamin-K inhibitor.

Conclusion: We question the possibility that intravenous thrombolysis therapy may have induced the right carotid dissection in this patient suffering from fibromuscular dysplasia.

\section{P-008}

\section{Surgical treatment of moyamoya disease by direct EC-IC bypass}

\section{P. Dirks, M. Tymianski (Toronto, Ontario)}

Background: Moyamoya disease is a progressive occlusive disorder of large basal cerebral vessels. Its most common surgical treatment involves brain revascularization by indirect methods, an approach favoured in children because of their small donor vessels and the belief that large recipient cerebral branches have been obliterated. We have performed EC-IC bypass in two children and three adults with moyamoya disease and report our early experience of the rationale and feasibility of direct revascularization in moyamoya disease by EC-IC bypass.

Methods: Review of moyamoya disease patients treated in the past two years at the Hospital for Sick Children and the Toronto Western Hospital.

Results: Since 1999 we have treated four children (seven hemispheres) and three adults (three hemispheres) with moyamoya disease. Pial synangiosis was performed early in the series in four hemispheres in children because of concern for lack of sufficient recipient vessels demonstrated by angiography. We recently attempted EC-IC bypass for three hemispheres in two children. EC-IC was feasible in children with donor and recipient vessels greater than $1 \mathrm{~mm}$ in diameter. EC-IC was feasible in all three adults because of sufficient caliber of vessels.

Conclusion: Adults and some children with moyamoya disease have cerebral vessels capable of receiving a direct EC-IC bypass even if these vessels cannot be seen on angiography.
Children with moyamoya disease are candidates for EC-IC bypass and a decision to perform the bypass can be made if donor and recipient vessels are greater that $1 \mathrm{~mm}$ in diameter.

\section{P-009}

\section{Nonatherosclerotic fusiform cerebral aneurysms}

\section{J. Max Findlay, C. Hao, D. Emery (Edmonton, Alberta)}

Background: Fusiform cerebral aneurysms are dilatations of the entire circumference of a segment of cerebral artery, usually considered due to atherosclerosis in adults, relatively thickwalled and elongated, and causing neural compression or ischemia when discovered. We have noted a subset of fusiform cerebral artery aneurysms that vary from this common description.

Patients: Out of a series of 472 intracranial aneurysms treated over 11 years, 11 patients between the ages 16 and 67 (mean age 37) were identified who had discrete fusiform aneurysms unassociated with generalized cerebral atherosclerosis, connective tissue disorder or inflammation. Three presented with hemorrhage, six with neural compression due to the aneurysm, and two were incidental discoveries.

Results: Nine aneurysms were located in the posterior circulation, the other two the intracranial carotid artery, their mean length and width 13 and $8 \mathrm{~mm}$, respectively. Two aneurysms contained thrombus. The eight aneurysms that were exposed surgically were partly or substantially thin-walled with normal parent arteries. Eight were treated with parent artery occlusion and three were circumferentially wrapped. Proximal occlusion caused one death and one mild disability, and the remaining patients made good recoveries (follow-up 1-9 years). Three-dimensional vascular imaging has recently proven useful in the recognition of this type of aneurysm.

Conclusion: There is a subset of cerebral aneurysms with a discrete fusiform morphology, apparently unrelated to cerebral atherosclerosis or systemic connective tissue disease, thinwalled in part or in whole, much commoner in the vertebrobasilar system, and possessing a risk of rupture. Treatments currently available include parent artery occlusion or aneurysm "wrapping", quite different approaches than neckclipping or endovascular coiling of side-wall saccular cerebral aneurysms.

\section{P-010}

\section{The Third International Stroke Trial (IST-3) - Thrombolysis for acute ischemic stroke}

\section{G. Gubitz (Halifax, Nova Scotia) for the IST-3 Collaborative Group}

Background: Thrombolytic therapy for acute ischemic stroke is the subject of considerable world-wide debate. The IST-3 will clarify the time window for treatment with tissue plasminogen activator (tPA), and more than double the total amount of available trial data.

Methods: The IST-3 is an ongoing international, doubleblind, randomized controlled trial evaluating intravenous tPA $(0.9 \mathrm{mg} / \mathrm{kg}-90 \mathrm{mg}$ maximum) versus placebo control in 6,000 
patients within six hours of stroke onset. Patients are eligible if over 16 years old with acute stroke of any severity, and if CT scan excludes intracranial hemorrhage. There must be no clear indication or contra-indication to treatment with tPA. Standard exclusion criteria (bleeding risk) apply. After consenting, patients are randomized using a central computerized telephone service. Patients must avoid antithrombotics for 24 hours, and have a repeat CT scan. Other aspects of patient care are at the physician's discretion.

Results: Outcome assessments will be blinded, and all analyses 'intention-to-treat'. Primary analysis: proportion of patients dead or dependent at six months (modified Rankin scale). Secondary outcomes include clinical events within seven days. All CT images will be reviewed (blindly) by an international panel of independent readers.

Conclusion: The IST-3 pilot phase is ongoing. For further information, visit the IST-3 website: www.den.ed.ac.uk/IST3

\section{P-011}

\section{The utility of the Oxfordshire Community Stroke Project (OCSP) stroke classification on a stroke unit}

G. Gubitz, S. Phillips, J. Jarrett, C. Christian (Halifax, Nova Scotia)

Background: The Oxfordshire Community Stroke Project (OCSP) classification of stroke subtype is pragmatic, and predicts clinical outcome in community-based settings. We studied the utility of the OCSPon our Stroke Unit, hypothesizing that OCSP sub-type would be predictive of death.

Methods: Our Stroke Unit provides organized stroke care for approximately 500,000 people. Information about age, gender and OCSPsub-type was prospectively gathered and analyzed for each patient with ischemic stroke admitted between January 1997 and December 1999. Using information from the database and the Nova Scotia Department of Health, we also determined which patients had died.

Results: During the study period, 183 of 680 ischemic stroke patients died $(27 \%)$. More men than women died (56\% versus $44 \% ; \mathrm{p}=0.2$ ). Patients with larger strokes by OCSP classification were more likely to die. Overall, $43 \%$ of patients with total anterior circulation strokes died, compared with $25 \%$ of partial anterior circulation strokes, $17 \%$ of lacunar strokes, and $16 \%$ of posterior circulation strokes. This trend was similar across all age strata.

Conclusions: The OCSP classification of stroke sub-type can be used to predict mortality for patients managed on a Stroke Unit. This may be useful in clinical care and in stroke service organization.

\section{P-012}

\section{Neurological outcome and quality of life after stroke due to vertebral artery dissection}

\section{Michael D. Hill, Diana Czechowsky (Calgary, Alberta)}

Background: Vertebral artery dissection is a well-recognised cause of posterior circulation stroke for which there is relatively little information on long term outcomes. Quality of life is an important patient-centred outcome measure.
Methods: Stroke due to vertebral artery dissection was conservatively defined by neuro-imaging documentation. Patient identification was based on a retrospective database and chart review with prospective follow-up. The Short-Form 36 (SF-36) and the Stroke-specific quality of life (SSQOL) scales were used to assess quality of life. Patients were examined neurologically and scored on the National Institutes of Health Stroke Scale and Canadian Neurological Scale.

Results: There was discordance between outcomes recorded on standard stroke scales and quality of life measures with more patients scoring poorly on QOL measures. QOL was low in one third of the survivors. Overall QOLwas significantly lower than the general population.

Conclusions: Stroke due to vertebral artery dissection results in poorer outcomes on patient-centred quality of life measures than on standard stroke scales.

\section{P-013}

\section{Paradigm shift in the treatment of intracranial aneurysms: the Halifax experience}

R.O.Holness, B. Addas, R. von Ritschl, R. Vandorpe, W. Maloney (Halifax, Nova Scotia)

Background: Despite improvement in microsurgical techniques significant morbidity still occurs from the rupture and treatment of intracranial aneurysms. Coil embolization for many aneurysms has attained credibility as an option to surgery in many centers. Since 1997, we have offered this method of treatment as the favored option to all patients in whom their aneurysm morphology satisfied accepted criteria for coiling.

Methods: We report 200 consecutive patients, $60 \%$ of whom had coil embolization as the primary treatment for their aneurysms. $50 \%$ of our cases presented with subarachnoid hemorrhage. Anterior communicating, internal carotid and basilar tip aneurysms comprised the vast majority of those who were coiled. We favor surgical clipping for middle cerebral aneurysms. Failed attempts or post coiling hemorrhage occurred rarely. Aneurysms ruptured during coiling in three cases.

Results: In patients with ruptured aneurysms, lengths of hospital stay and neurological outcome were similar regardless of method of treatment. However Grades 1 and 2 SAH cases whose aneurysms were coiled had a shorter ICU stay and there was a trend towards a more favorable outcome in the elderly. Lengths of stay for patients with unruptured aneurysms were significantly shorter for those treated with coils. Follow-up angiography and clinical data will be presented.

\section{P-014}

\section{Recurrence of Susac Syndrome after many years of remission}

\section{T. Lad, C. Boyle (Saskatoon, Saskatchewan)}

We report a case of recurrent Susac syndrome, reoccurring 19 years after initial presentation. Susac syndrome is characterized by a recurrent course with a clinical triad of hearing loss, encephalopathy and retinopathy. Susac syndrome is usually self limited, however neurological deficits may persist. Frequency of 
recurrent attacks is reported to be between 1-8. Intervals of longer than three years between recurrences have not been described. This case of Susac syndrome demonstrates that recurrence may occur as many as 19 years after initial presentation.

\section{P-015}

\section{Coordinated stroke strategy - an organized system for stroke care}

\section{Lewis, B. Powell-Vinden (Toronto, Ontario)}

Background: The Coordinated Stroke Strategy (CSS) is a successful demonstration project that has improved access to organized stroke care in four regions within Ontario by implementing a comprehensive stroke strategy and obtaining government commitment. In the fall of 1998, the Heart and Stroke Foundation of Ontario launched CSS to pilot regional organized stroke care across the continuum of care.

Methods: The CSS has three strategic components: systems change, professional education and public awareness. Network development, protocol implementation, professional education and public awareness activities are underway in each site and have improved access to evidence-based interventions such as tPA. Provincially, the strategy has engaged the Ministry of Health and Long Term Care (MOHLTC) for equitable access to stroke care.

Results: The implementation of regional stroke protocols has resulted in more patients receiving tPA within three hours. The MOHLTC has committed $\$ 30$ million for the development of a province-wide strategy. A multi disciplinary consensus panel has gathered evidence on stroke rehabilitation and developed a system.

Conclusions: The Coordinated Stroke Strategy is a first ever comprehensive strategy for organized stroke care in Canada. It has demonstrated that a coordinated strategy can mobilize regional networks and engage government to enhance patient care.

\section{P-016}

\section{Proton magnetic resonance spectroscopy (MRS) in differentiating a stroke versus tumor. Is this a worthy clinical application?}

V. Mehta, P.D. McNeely, R. VonRitschl, L. Gates (Halifax, Nova Scotia)

Background: MRS can yield biochemical information that may be helpful in the clinical management of neurosurgical patients. Much has been written about the usefulness of an increased choline peak, decreased creatine peak and altered $\mathrm{N}$ acetyl aspartate (NAA) peak in diagnosing brain tumors. Differentiating a neoplasm from a stoke centers around the loss of the NAA peak and a normal choline peak.

Methods: We were recently involved in the management of two interesting cases where standard MRI imaging (with diffusion weighting) was unable to differentiate definitively a stroke versus tumor. The first case involved a 63-year-old with headaches and a visual field deficit where MRI imaging demonstrated a gyral enhancing lesion within a watershed area. Case two involved a 64-year-old surgeon who presented with foot numbness within a month of cardiac surgery. A lesion involving the medial primary sensory strip was found.

Results: When faced with the clinical situation of a stroke versus a tumor, a wait and see approach may be adopted. Serial imaging will either demonstrate volume loss with a stroke or radiographic progression with a tumor. The lack of a choline peak in our MRS study gave us further evidence to avoid an invasive procedure and follow the patient radiographically. Eight month follow-up data on these cases will be presented.

Conclusions: MRS may be a useful test in differentiating a tumor versus stroke in everyday clinical management.

\section{P-017}

\section{Susac's syndrome (retinocochleocerebral} microangiopathy): report of three cases

\section{A. Nadeau, S. Gosselin, J.P. Bernier, F. Evoy (Sherbrooke, Quebec)}

Susac's syndrome is a rarely-reported microangiopathy of the brain, retina and cochlea of uncertain etiology affecting mostly women in their third and fourth decades.

We report three patients with the disease: two men (33 and 40-year-old) and one woman (37-year-old). The two men presented with sub-acute encephalopathy with neurocognitive impairment and focal neurologic deficits and developed neurosensorial deafness within a few weeks but no visual symptoms. The woman first experienced visual loss and ataxia followed by diffuse encephalopathy and deafness two months later.

In all three patients, the brain MRI showed multifocal hyperintensities on T2-weighted images; normal cerebral angiogram; increased spinal fluid protein; multiple retinal artery narrowings on retinal angiography; and small foci of necrosis without vasculitic changes on brain biopsy. All investigations for infectious diseases, autoimmune disorders or coagulopathy were negative.

The encephalopathy of the three patients improved with aspirin and immunosuppressive treatment but it did not prevent the appearance of deafness in two patients.

Susac's syndrome is probably more frequent than reported and should be suspected in young patients with subacute encephalopathy with or without focal neurologic deficits. Retinal angiography may help make the diagnosis early, even in the absence of visual symptoms.

\section{P-018}

\section{Cerebral amyloid angiopathy presenting as a non- hemorrhagic mass lesion}

\section{A. Quirion, J.B. Lamarche, S. Jarjoura (Sherbrooke, Quebec)}

Background: The clinical and radiological presentation of cerebral amyloid angiopathy (CAA) as a nonhemorrhagic cerebral mass lesion of the white matter has rarely been described. 
Methods: We report the case of a 83-year-old female with a symptomatic cerebral nonhemorrhagic mass lesion. A postmortem examination provided the diagnosis of CAA.

Results: The patient presented a rapidly progressive dysphasia, cognitive decline and a tonic-clonic seizure. The MRI showed a left fronto-parietal nonenhancing lesion with mass effect. A low grade glioma or an ischemic event was considered. She remained dysphasic and died of pulmonary emboli two weeks later. An autopsy revealed an ill-defined whitish mass in the left fronto-parietal cerebral white matter, which consisted of marked chronic interstitial edema associated with myelinoaxonal loss. Widespread CAA was found in the small leptomeningeal and cortical arteries. Petechial hemorrhages and microscopic infarcts of different ages were noted in the frontal cortex bilaterally.

Conclusions: Nonhemorrhagic mass lesion should be added to the clinical and radiological spectrum of presentation of CAA.

\section{P-019}

\section{Multidisciplinary treatment of dural arteriovenous fistulae with retrograde venous drainage}

\section{G. Redekop, T. Marotta, A. Weill (Vancouver, British Columbia)}

Introduction: Intracranial dural arteriovenous fistulae (DAVFs) with retrograde leptomeningeal venous drainage are associated with an aggressive clinical course. To evaluate the safety and efficacy of a treatment strategy based on disconnection of venous drainage, we reviewed our experience using a multidisciplinary surgical and endovascular approach.

Methods: Thirteen patients with Borden Grade 2 and 3 DAVFs were treated in the past two years. Fistulae were located in the region of the transverse sinus in six cases (46\%), the superior petrosal sinus in five $(38 \%)$, and one each in the cavernous sinus and cribriform plate. Details of clinical presentation, lesion angioarchitecture, treatment, post-treatment angiography, and clinical outcome were analyzed.

Results: Hemorrhage was the presenting symptom in two of four (50\%) Grade 2 and seven of nine (77\%) Grade 3 DAVFs. One patient died from recurrent bleeding prior to intervention. Embolization via a transarterial or transvenous approach was used in eight cases. Two patients had complete elimination of their DAVF following transarterial liquid adhesive embolization. Four had transarterial embolization for pre-surgical devascularization, and two had transarterial embolization and transvenous sinus occlusion with near-complete elimination of their fistulae. Seven patients had surgical disconnection of retrograde leptomeningeal venous drainage and one had isolation of the transverse and superior sagittal sinus. There were no hemorrhagic or ischemic complications. Follow-up angiography confirmed complete elimination of the DAVF in all of the Grade 3 DAVFs (with direct leptomeningeal venous drainage) but only one Grade 2 lesion. One patient with subtotal fistula occlusion experienced late rebleeding because of recurrence.

Conclusion: DAVFs with direct retrograde leptomeningeal venous drainage are safely and effectively treated with venous disconnection alone. Those with venous sinus involvement recur if the sinus is not obliterated.
P-020

\section{Treatment of traumatic skull base vascular lesions with endovascular stent grafts}

\author{
G. Redekop, T. Marotta, A. Weill (Vancouver, British \\ Columbia)
}

Background: We present our preliminary clinical experience with endovascular stents for traumatic vascular lesions of the skull base region. Because adequate distal exposure and direct surgical repair of these lesions are not usually possible, conventional treatment has been deliberate arterial occlusion. The purpose of this report is to demonstrate the safety and efficacy, as well as limitations, of endovascular stent deployment in the management of craniocervical arterial injuries.

Methods: Six patients with vascular injuries were treated with endovascular stents. There were two arteriovenous fistulae and two pseudoaneurysms of the distal extracranial internal carotid or vertebral arteries resulting from penetrating trauma, and two petrous carotid pseudoaneurysms associated with basal skull fractures. One patient was treated with a porous stent as well as coil occlusion of an aneurysm, while the remaining five had covered stent grafts as definitive treatment.

Results: There were no procedural complications. One patient with extensive traumatic arterial dissection was found to have asymptomatic stent thrombosis when angiography was repeated one week postoperatively. This was the only patient whose associated injuries precluded routine antithrombotic or antiplatelet therapy. The remaining five patients underwent follow-up angiography three to twelve months postoperatively. In all five cases, the vascular injury was cured and the parent artery remained widely patent. No patient experienced recurrence or hemorrhage, and there were no thromboembolic complications.

Conclusions: Our experience demonstrates that endovascular treatment of traumatic vascular lesions of the skull base region is both feasible and safe. The advantages of minimally invasive deployment and parent artery preservation make this technique for repair of neurovascular injuries a potentially important addition to existing methods.

\section{P-021}

\section{Sustainability and transferability of a regional stroke program}

R.J. Riopelle (Montreal, Quebec), C. Bolton, S. Elson (Kingston, Ontario)

Background: Southeastern Ontario (SEO) has a population of 475,000 and encompasses an area of 20,000 square kilometres. In July 1999, a regional acute stroke protocol (RASP) was implemented to ensure equitable access to evidence-based acute stroke interventions. The RASP is the first regional stroke protocol in Canada. It was developed in partnership with the Heart and Stroke Foundation of Ontario (HSFO), with leadership from the Queen's University Care Delivery Network (CDN), a research and development project funded by an unrestricted grant from Glaxo Wellcome.

Methods: The CDN project team undertook a process analysis 
of the development and implementation of the RASPto examine factors contributing to its success and its applicability to related stroke initiatives.

Results: Based on experience to-date, the sustainability and transferability of initiatives like the RASP require regional planning and coordination, resources, education, and leadership across clinical disciplines and organizations. Partnerships with nongovernmental organizations (e.g., HSFO) provide public visibility, credibility and advocacy.

Conclusion: The twelve-month experience with the RASP has demonstrated that a coordinated stroke system improves access to acute stroke interventions, and outcomes of care. The process analysis revealed generic factors key to sustaining and transferring the RASP to other stroke initiatives and conditions (e.g., cardiac).

\section{P-022}

\section{A scientific approach to increasing awareness levels of the signs and symptoms of stroke}

\section{Frank D. Rubini (Toronto, Ontario)}

Background: The Coordinated Stroke Strategy is a three-year, multi-site demonstration project led by the Heart and Stroke Foundation of Ontario to test improved stroke intervention across the continuum with a goal to ensure equitable access to the best possible stroke care. One of the three major components to the Coordinated Stroke Strategy includes public awareness, focused on establishing top of mind awareness of the signs and symptoms of stroke so that individuals can get to hospital immediately.

The other two components are health systems change and professional health care education.

Methods: Develop and implement a scientifically accurate system to measure the impact of a mass media and public relations campaign designed to increase the awareness levels of the signs and symptoms of stroke amongst adults 45 years of age and older.

Results: There has been a statistically significant increase in the levels of awareness of the signs and symptoms of stroke. Test areas utilizing television, coupled with a public relations component did better than other forms of advertising.

Conclusions: With the proper combination of paid and unpaid communications vehicles, it is possible to dramatically increase awareness levels of strokes signs and symptoms.

\section{P-023}

\section{Should intravenous heparin be stopped prior to carotid endarterectomy?}

\section{J.A. Salame, P. Couillard (Sherbrooke, Quebec)}

Background: It is customary to interrupt intravenous (IV) heparin four hours preoperatively in order to avoid hemostatic problems during carotid endarterectomy. However, this practice is not substantiated by medical evidence. Our aim in this study was to reassess this practice and evaluate the safety of uninterrupted IV heparin prior to surgery.

Methods: The medical records of 34 consecutive patients who underwent carotid endarterectomy were available for retrospective review. Twelve of the 34 patients were under IV heparin preoperatively because of an unstable neurological status. In these 12 patients, heparin perfusion was continued throughout surgery and patients received intraoperative heparin as well. We reviewed in all cases the intraoperative blood loss, the length of surgery, hospital stay, and the incidence of new neurological deficit and neck hematoma.

Results: No significant difference existed between the two groups of patients for the above variables.

Conclusion: Intravenous heparin does not have to be discontinued prior to carotid endarterectomy.

\section{P-024}

\section{Symptomatic and angiographic follow-up in benign} intracranial dural arteriovenous fistulas

J. Satomi, K. terBrugge, R. Willinsky, W. Montanera, M. Tymianski, P. Porter, M.C. Wallace (Toronto, Ontario)

Background: We evaluated the chronological change in symptoms and angiographic features in patients with intracranial dural arteriovenous fistulas (DAVFs) without retrograde leptomeningeal venous drainage (RLVD) followed conservatively.

Methods: Seventy-two DAVFs with sinosal venous drainage only (Borden classification type 1 [J Neurosurg 1995;82:166]) presented with benign symptoms/signs. Forty-six cases were observed. Transarterial embolization for symptom palliation, not angiographic cure, was used in 26 cases. Mean follow-up term was 32 months (180 patient years). Angiography was performed in 32 cases (observation group: 16, embolization group: 16). We compared the symptoms and angiographic features in these DAVFs between initial assessment and follow-up.

Results: Symptomatic improvement was detected in $74 \%$ (35/46) of observation group and $96 \%$ (25/26) of embolization group. One case showed intraparenchymal bleeding in followup. In patients having follow-up angiography, shunt disappearance was detected in $47 \%$ (15/32), shunt reduction in $25 \%$ (8/32), no change in $22 \%$ (7/32). Two cases (transversesigmoid sinus with improvement in symptoms; cavernous sinus with worsening symptoms-intraparenchymal bleeding) demonstrated spontaneous conversion of a lesion with sinosal drainage only into one with RLVD associated with progressive sinus thrombosis.

Conclusion: The natural history of hemorrhage/infarction of intracranial DAVF with sinosal venous drainage only is extremely benign. However, benign DAVFs have the potential risk of angiographic conversion to an aggressive type, requiring close clinical follow-up and radiological evaluation for an improvement or deterioration in symptoms.

P-025

\section{Natural history of a cavernous malformation followed through pregnancy}

S.K. Singh, F. Meffe, M.D. Cusimano (Toronto, Ontario)

Little is known about the natural history of cavernous 
malformations during pregnancy. An appraisal of the literature reveals no conclusive data on whether pregnancy increases the risk of hemorrhage from these vascular malformations. We review the physiological impact of pregnancy on the natural history of cavernous malformations, as documented by serial MR imaging of the brain. We report the case of a 37-year-old G3P2A1 female who was diagnosed with a left middle cerebellar peduncle cavernous malformation following a single episode of hemorrhage marked by fluctuating symptomatology. She was managed conservatively and her symptoms resolved over time. Three years and two months after her initial presentation, she became pregnant, and did not suffer any subsequent episodes of rebleeding from her cavernous malformation throughout the course of her pregnancy and delivery. Serial MR imaging, including MRI of the brain performed within 24 hours of delivery, is assessed in the context of this patient's conservative management. We review the literature on cavernous malformations and pregnancy, and discuss management options as dictated by natural history.

\section{P-026}

\section{Use of an intravenous heparin bolus in the initial} anticoagulation of patients with transient ischemic attack or stroke - subgroup analysis of the HANST study

\section{Toth, C. Voll (Saskatoon, Saskatchewan)}

Objective: To examine the clinical and laboratory results of the use of an intravenous heparin bolus during initiation of anticoagulation therapy in patients with transient ischemic attack (TIA) or stroke.

Background: Intravenous heparin therapy is often used in patients presenting with TIAor stroke as either bridging therapy for anticoagulation with coumadin, or as primary therapy in suspected intracranial arterial dissection, crescendo TIAs, or suspected hypercoagulable states. There is no evidence that an initial heparin bolus is dangerous, but many neurologists have an apprehension of this practice.

Methods: Subgroup analysis of patients enrolled in the HANST study was performed. This study was not designed to examine efficacy of heparin therapy, but to examine safety and accuracy of a heparin bolus.

Results: A total of 206 patients were enrolled and randomized in the HANST study. Of these patients, 33 patients received a heparin bolus averaging $4500 \mathrm{U}$, and 173 patients received no bolus. The two groups were not equivalent with regards to indication for heparin therapy, as more patients in the bolus subgroup had a suspected cardioembolic source. Initial maintenance heparin infusion rates were equal between the two groups. Patients receiving a heparin bolus had a significantly higher initial partial thromboplastin time (PTT) than nonnomogram patients (Mann-Whitley, $\mathrm{p}<0.01$ ). Patients receiving boluses had a significantly greater probability of achieving minimum therapeutic anticoagulation levels earlier (t-test, $\mathrm{p}<0.01$ ). There was no significant difference in the number of supratherapeutic coagulation results, number of complications, time to discontinue heparin, and time to discharge patients.

Conclusion: The practice of an initial intravenous heparin bolus in TIA or stroke may be associated with a faster time to achieve minimal threshold for anticoagulation and may not be associated with a greater risk of complications. This result is limited by the subgroup inequality in this study. A randomized study would be required to further investigate the practice of heparin bolusing.

\section{P-027}

Treatment of acute basilar artery thrombosis with local intra-arterial thrombolysis, angioplasty and endovascular stenting: a case report

\section{S. Verreault, J-L. Gariépy, A. Mackey (Quebec City, Quebec)}

Background: Basilar artery (BA) thrombosis has a very poor prognosis. Local intra-arterial thrombolysis (LIAT) of acute BA thrombosis has been shown to reduce mortality. We report a case of acute BA thrombosis where combined treatment with LIAT, angioplasty and endovascular stenting were performed.

Case Report: A 60-year-old male presented with a decreased level of consciousness of acute onset, rapidly requiring endotracheal intubation. Neurological examination revealed absent ocular movements, left hemiparesis and bilateral extensor plantar responses. Cerebral angiography done five hours after symptoms onset showed a BA occlusion. LIAT with t-PA reopened the BA and permitted visualization of a $95 \%$ mid-BA stenosis. Angioplasty was performed and two endovascular stents were inserted. Cerebral angiography control showed a complete recanalization of the BA. Signs of ischemia in the pons, cerebellum, occipital lobes and left thalamus were identified on MRI done the next day. At six months the patient was at home using a wheelchair with a NIHSS of 7 and a Barthel index of 75 .

Conclusion: In light of the devastating natural course of acute BAthrombosis, this case suggests that combined treatment with LIAT, angioplasty and endovascular stenting may be an effective emergency therapy resulting in reduced mortality and morbidity.

\section{P-028}

\section{Stroke and late onset migraine}

Nada Vujovic Zotovic, Na Jiang, John Norris (Toronto, Ontario)

Background: Migraine attacks may be accompanied by a variety of transient neurological symptoms, mainly visual, but when occurring in late life may be confused with atherothrombotic acute ischemic events.

Methods: In our Stroke Prevention Clinic, we evaluated the files of 718 patients presenting with TIA or stroke over a 3-year period. $79(11 \%)$ patients had clinical symptoms of migraine. We excluded 26 because of alternative causes of stroke or inadequate data collection. The remaining 53 were divided into 26 with visual symptoms only and 27 with predominantly neurological symptoms. Diagnosis was based upon clinical and laboratory testing including $\mathrm{CT}$ and MR scans, angiograms and echocardiograms.

Results: In the neuro group, age ranged from 27-87 years (mean 53). Mean duration of migraine was 24 years (range 1-75 years). There were four strokes, all vertebrobasilar and 23 TIAs. No distinct carotid symptoms were seen and in 18 (of 23) typical 
visual scintillations occurred. Two patients presented migrainous TIAs for the first time, aged 57 years. In the visual only group, the presentation of the symptoms had changed and the patients were older (mean age 58+14years, 28-85 years).

Conclusion: Patients with late onset migraine may be unnecessarily investigated when a careful clinical history will suffice. Whether migraine produces ischemic stroke or viceversa is more difficult to determine.

\section{DEMENTIA}

\section{P-029}

\section{Goal setting in Alzheimer's disease treatment: do physicians and patients/caregivers agree?}

R. Bassett, K. Rockwood, S. Fay, C. Wentzel (Halifax, Nova Scotia), J. Graham (Vancouver, British Columbia).

Background: Goal Attainment Scaling (GAS) is a clinimetric measure to evaluate patients with multidimensional illnesses and variable treatment responses. We report the extent to which physicians and patients/caregivers set similar goals and the use by both of maintenance as a treatment outcome.

Method: In an open-label, prospective, descriptive, Phase IV trial of donepezil, baseline goals were established by physician and patient/caregiver. Analyses of number of goals (total and by domain) and distribution of maintenance goals were performed.

Results: For 100 patients, physicians set 342 baseline goals in cognition, 117 (34\%); function, 108 (31\%); behaviour, 73 (21\%); social interaction, 24 (7\%); leisure, $20(6 \%)$. Patients/caregivers set more goals (855): in function, $255(30 \%)$; leisure, $218(25 \%)$; cognition, 209 (24\%); behaviour, 93 (11\%) and social interaction, 80 (9\%). These proportions were significantly different from the physician profile within the domains of cognition $(\mathrm{p}<.0002)$, behaviour $(\mathrm{p}<.0002)$ and leisure $(\mathrm{p}<.0001)$. Patients/caregivers also set more maintenance goals $(24 \%$ vs $2 \% ; \mathrm{p}<.001)$.

Conclusion: There is substantial overlap in the goals of physicians and patients/caregivers. Compared with the latter however, physicians set fewer goals, particularly less often in leisure, and are less likely to view maintenance as the purpose of treatment.

\section{P-030}

Butyrylcholinesterase and acetylcholinesterase in the human intrinsic cardiac nervous system: implications in Alzheimer's disease

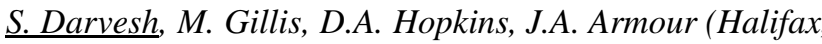 Nova Scotia)}

Background: Cholinesterase inhibitors are widely used to treat the symptoms of Alzheimer's disease (AD) by inhibiting the enzymes acetylcholinesterase (AChE) and butyrylcholinesterase (BuChE). These enzymes also play an important role in the intrinsic cardiac nervous system (ICNS) that regulates the heart. Patients with AD display cardiac autonomic dysfunction.
Whether this autonomic dysfunction is partly due to pathological changes in the ICNS is unknown. Therefore, to establish baseline information we examined the distribution of these enzymes in the human ICNS.

Materials, Methods: Sixteen intrinsic cardiac ganglia were harvested from a normal human heart at autopsy and processed for $\mathrm{AChE}$ and BuChE histochemical staining.

Results: Human intrinsic cardiac ganglia contain neurons that express both $\mathrm{AChE}$ and $\mathrm{BuChE}$ in both their soma and processes. The cellular distribution of these enzymes differed. AChE was found in the cytoplasm and the nucleus, while BuChE was predominantly in the cytoplasm.

Conclusions: The observation that the human ICNS contains neurons that express both $\mathrm{AChE}$ and $\mathrm{BuChE}$ supports the notion that both enzymes are important in the cholinergic control of cardiac neurons. This has major implications with respect to the treatment of AD because of the potential effects of cholinesterase inhibitors on the intrinsic cardiac neurons, particularly with regards to cardiac arrhythmic formation.

\section{P-031}

\section{Enzyme kinetic properties of cholinesterase inhibitors used to treat Alzheimer's disease}

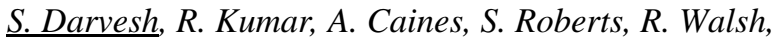
E. Martin (Halifax, Nova Scotia).

Introduction: In Alzheimer's disease (AD), there is a reduction in the neurotransmitter acetylcholine (ACh) and its hydrolyzing enzymes acetylcholinesterase (AChE), but an increase in butyrylcholinesterase (BuChE). Clinical efficacy of cholinesterase inhibitors (ChE-I) used to treat the symptoms of $\mathrm{AD}$ is thought to be due to inhibition of AChE. Enzyme kinetic data for inhibition of cholinesterases is derived using enzymes from a variety of sources, and there are marked differences in the specificity ChE-I have toward cholinesterases from different sources. We determined the specificity and enzyme kinetic properties of ChE-I used for the treatment of $\mathrm{AD}$ towards human cholinesterases.

Materials, Methods: The esterase activity of human serum BuChE and erythrocyte AChE was studied using a modified Ellman's spectrophotometric method. Inhibition constants (Ki) for donepezil, galantamine, huperzine A, rivastigmine, and tacrine were determined.

Results: Donepezil, galantamine, rivastigmine, and tacrine inhibited both $\mathrm{AChE}$ and BuChE. Huperzine A was specific for $\mathrm{AChE}$. The inhibitor constants for different drugs varied as much as 100-fold between $\mathrm{AChE}$ and $\mathrm{BuChE}$, some being better inhibitors of one or the other enzyme.

Conclusions: Donepezil, galantamine, and rivastigmine, drugs currently being used to treat $\mathrm{AD}$, inhibit both $\mathrm{AChE}$ and BuChE. Huperzine A inhibits only AChE and its clinical use remains to be determined. Inhibition of $\mathrm{BuChE}$ and $\mathrm{AChE}$ appears to be important for treatment of AD. 
P-032

\section{Neuropsychological predictors of incident dementia in patients with vascular cognitive impairment, without dementia}

J. Ingles, C. Wentzel, J. Fisk, K. Rockwood (Halifax, Nova

Scotia)

Background: Vascular cognitive impairment that does not fulfill dementia criteria (referred to as vascular cognitive impairment, no dementia [CIND]) is common and progresses to dementia at a high rate. However, little is known about factors that predict progression of this diagnosis. We examined whether performance on neuropsychological tests administered at baseline could predict incident cases of dementia in patients with vascular CIND.

Methods: The Canadian Study of Health and Aging is a prospective, cohort study of 10,263 randomly selected persons aged 65 years or older. Our sample comprised 102 of these participants who were diagnosed with vascular CIND and who had completed a battery of neuropsychological tests at baseline. Participants were re-assessed five years after the original study.

Results: After five years, 45 (44\%) participants developed dementia. These participants performed significantly worse on tests of memory (Buschke Cued Recall Test) and word fluency (animal naming) at baseline compared to those who did not develop dementia (adjusting for age and education).

Conclusions: Individuals with vascular CIND who progress to dementia perform less well on neuropsychological tests at baseline. This suggests that it may be possible to identify those appropriate for preventative efforts.

\section{P-033}

\section{Caregiver burden in Alzheimer's disease: results from an open-label, randomized, multicenter clinical trial evaluating the effectiveness of donepezil hydrochloride vs donepezil with TriAD $^{\mathrm{TM}}$}

\section{Serge Gauthier (Verdun, Quebec), Isabelle Lussier (Kirkland, Quebec), the TriAD TM Study Group}

Caregiving is defined as the activities and experiences involved in providing assistance to someone who is unable to care for him- or herself. For a disease such as Alzheimer's, the role of the caregiver, who is typically a spouse or an adult child, centers around providing the patient with adequate supervision, financial security, personal care, support and companionship, ensuring the patient's well-being, and performing all household chores. These responsibilities can have a profound impact on the lives of caregivers. An important objective of this study is to determine the effects of donepezil hydrochloride and an education program on caregiver burden in Alzheimer's disease (AD). TriAD ${ }^{\mathrm{TM}}$ is a comprehensive $\mathrm{AD}$ management program for clinicians, patients and caregivers, designed to answer questions, describe disease progression, provide support and education, and promote a better understanding of what to expect and ways to better manage the symptoms of this disease. In this clinical trial, over 400 patients with mild to moderate, possible or probable $\mathrm{AD}$ (diagnosed in the previous 12 months) have been evaluated. All patients received a daily dose of donepezil hydrochloride of 5 or $10 \mathrm{mg}$. Half of the patients received the TriAD ${ }^{\mathrm{TM}}$ Disease Management Program from the first visit and for the following seven months, while the other half received standard care. Clinicians and caregivers were asked to give their global impression of change in the patient to evaluate overall tolerability and efficacy of treatment. The caregivers' level of knowledge about the illness, their ability to cope and their sense of burden were also assessed, and the Zarit caregiver burden scale was used to assess overall caregiver burden. Results showed that the TriAD ${ }^{\mathrm{TM}}$ program had a positive impact on the caregivers' knowledge about AD and their ability to cope with the role and responsibilities of caregiving, as well as on their sense of burden. Both the caregivers and the investigating physicians expressed a high degree of satisfaction with the medication and the TriAD ${ }^{\mathrm{TM}}$ program, which met the specialist care needs of both AD patients and their caregivers, bringing a feeling of support and guidance to both.

\section{NEUROSCIENCE}

\section{P-034}

\section{Early human spinal cord development}

A.M. Belkadi, I. Mendez (Halifax, Nova Scotia)

Background: The general anatomical development of the human spinal cord is well studied. However, the development of the chemical neuroanatomy of the spinal cord remains largely unknown. Our goal was to characterize the neuronal differentiation of different neurotransmitter phenotypes in the developing cervical spinal cord.

Methods: Human specimens ranging from 7 to 9.2 weeks postgestation were studied. The tissue was sectioned on a freezing microtome and processed immunocytochemically. Polyclonal antibodies including nestin, protein gene product 9.5 , glutamate receptor 2 and 3 and $67-\mathrm{kDa}$ glutamic acid decarboxylase were used for neurochemical characterization.

Results: A prominent nestin-labeled ependymal zone of progenitor cell processes, which mainly expressed first in the dorsal cord from the 7 th to 8 th gestational weeks, then in the ventral cord from the eight and half to the ninth weeks was observed. These ventral progenitor cells migrating towards the gray matter differentiated mainly into a neuronal phenotype by the 8th gestational week with a formation of gray matter nuclei. The dorsal nestin-positive fibers zones displayed a few differentiated neurons. However, with the down regulation of the nestin protein from eight weeks onward, the dorsal and ventral horns show an increase in the number of neurons which express PGP9.5, GluR2/3 and GAD67 antigens at their final localization.

Conclusion: By the 8th gestational week, progenitor cells, which differentiate into neurons mainly in the ventral horn are prominent in the ependymal zone. Those neurons expressing PGP9.5, GluR2/3 and GAD67 antigens show a mature phenotype at final localization. 


\section{P-035}

\section{Chronic endothelin antagonism restores cerebral vascular function in experimental diabetes}

Aaron S. Dumont, Neal F. Kassell (Charlottesville, Virginia, USA), Randall J. Dumont, John H. McNeill (Vancouver, British Columbia), Garnette R. Sutherland (Calgary, Alberta), Duncan J. Stewart, Subodh Verma (Toronto, Ontario)

Background: Diabetes profoundly alters vascular function. Diabetes increases myogenic tone and decreases responsiveness to ATP sensitive $\mathrm{K}^{+}$channel $\left(\mathrm{K}_{\text {ATP }}\right)$ openers and endotheliumdependent vasodilators. The mechanism(s) by which diabetes impairs cerebrovascular function remain obscure.

Methods: In the present study, we examined the effects of the potent vasoactive peptide, endothelin-1 (ET-1), on myogenic tone and endothelium-dependent and potassium channel mediated vasodilation in middle cerebral arteries (MCA) from diabetic and nondiabetic rats. Twenty-eight Wistar rats were divided into four experimental groups ( $\mathrm{n}=7$ per group): control [C], control bosentan ( $\mathrm{ET}_{\mathrm{A} / \mathrm{B}}$ antagonist)-treated [CB], diabetic [D] and diabetic bosentan-treated [DB]. Diabetes was induced with streptozotocin (STZ) (D and DB groups) following which chronic bosentan treatment was initiated (CB and DB groups). MCA were mounted in a pressure myograph and myogenic responses were recorded. In addition, endothelium-dependent and -independent responses and the effects of the $\mathrm{K}_{\text {ATP }}$ channel opener, pinacidil, were examined.

Results: Cerebral arteries from the diabetic and nondiabetic rats constricted in response to graded pressure increases. Maximum myogenic responses (\% constriction at $60 \mathrm{~mm} \mathrm{Hg}$ ) were significantly greater in the D group ( $38 \pm 3 \%$ vs. C $25 \pm 3 \%$, $\mathrm{p}<0.02)$. The enhanced myogenic tone in the $\mathrm{D}$ group was completely prevented by bosentan treatment (DB $23 \pm 5 \%$ vs. D, $\mathrm{p}<0.003$ ) without an effect on the CB group. Additionally, bosentan treatment improved endothelium-dependent vasomotion and improved $\mathrm{K}_{\text {ATP }}$ mediated vasodilation in the DB group $(\mathrm{p}<0.001)$.

Conclusions: These data describe for the first time the interaction between ET-1, myogenic tone and endothelial function in diabetes. Chronic endothelin antagonism restores cerebral vascular function in this model of diabetes.

\section{P-036}

\section{Administration of BDNF alters the expression of Hsp27 in retinal ganglion cells following axotomy}

A.M.R. Krueger-Naug, J.G. Emsley, T.L. Myers, R.W. Currie, D.B. Clarke (Halifax, Nova Scotia)

Background: Optic nerve transection results in the apoptotic cell death of up to $95 \%$ of retinal ganglion cells (RGCs) after 14 days. It has been shown that Hsp27, with its anti-apoptotic effects, may be important for cell survival following axotomy or trophic factor withdrawal. In addition, it is known that the neurotrophin brain-derived neurotrophic factor (BDNF) enhances survival of RGCs. Recently, we reported the induction and expression of the small heat shock protein Hsp27 in RGCs following axotomy. Here we examined the effect of BDNF administration on the expression of Hsp27 in axotomized adult rodent RGCs.

Methods: RGCs were pre-labelled with fluorogold prior to optic nerve transection and concomitant intraocular injection of BDNF or vehicle (control). Hsp27 immunohistochemical staining in retinal sections from four to 28 days following injury was examined.

Results: Consistent with previous survival studies, the number of fluorogold-labeled RGCs declined from $100 \%$ at four days to $10 \%$ by 14 days following axotomy and vehicle injection. In contrast, with BDNF administration, RGC survival is maintained at $100 \%$ to seven days following axotomy. Hsp27 immunohistochemical staining indicates that the number of Hsp27 positive injured RGCs is decreased by $75 \%$ in BDNFtreated retinas, when compared with controls. This decreased expression of Hsp27 in response to BDNF treatment is seen both at early (4 days) and delayed (10 days) times.

Conclusions: BDNF treatment following optic nerve transection significantly reduces the expression of $\mathrm{Hsp} 27$ in RGCs. These results indicate that BDNF may downregulate alternate cell survival pathways including the stress-induced expression of Hsp27.

P-037

Novel truncating germline mutation in VHLtumour suppressor gene presenting as type II Von Hippel Lindau syndrome

T.G. Mainprize, M.D. Taylor, R.A. Wennberg, J.T. Rutka, D. Chitayat (Toronto, Ontario)

Background: Von Hippel-Lindau (VHL) syndrome is an autosomal dominant disorder secondary to mutations in the VHL gene that presents with central nervous system hemangioblastomas, pheochromocytomas (type I without and type II with), renal cell carcinomas, and abdominal organ cysts. Type I VHLis associated with deletions or truncating mutations, whereas type II VHL is associated with missense mutations. We describe a patient with Type II VHL and a novel truncating germline mutation in the VHL gene. The VHL protein, along with Elongin $\mathrm{B} /$ Elongin $\mathrm{C} / \mathrm{Cul}$, regulate the level of VEGF.

Methods: A blood sample for DNA sequencing was obtained with permission from a 23-year-old patient with bilateral renal cell carcinomas, pancreatic and epididymal cysts, pheochromocytomas and multiple hemangioblastomas of the spinal cord. There was no family history of VHL.

Results: Sequencing of white blood cell DNA revealed a novel tetranucleotide insertional mutation at position 725 (Nt725TTGA) resulting in a K171N substitution and premature stop at codon 172. The second VHL allele was wild-type.

Conclusion: We describe a new truncating mutation in a patient with type II VHL syndrome. This mutation would partially disrupt the Elongin $\mathrm{C}$ binding site but may not totally abolish this necessary intermolecular association. 
P-038

\section{Spheroid growth and invasion in three-dimensional collagen gels \\ T. Werbowetski, R.F. Del Maestro (Montreal, Quebec)}

Background: A three-dimensional implantation model has been developed in which malignant glial tumor cells invade a collagen type I gel. This system has been used to assess the invasion of spheroids, aggregates of malignant glial cells, and human tumor explants. To further characterize malignant glial cells in this model, we have assessed the growth and invasive profile of implanted C6 (murine astrocytoma) and U251 (human malignant glioblastoma) spheroids.

Materials and methods: C6 and U251 spheroids were implanted into three-dimensional collagen type I gels (Vitrogen $100)$, and the spheroid growth and invasive parameters were monitored over 12-14 days.

Results: C6 spheroids initially expanded into the matrix over the first 5-6 days reaching a maximum diameter increase of $205 \pm 4.6$ and $320 \pm 45.0 \mu \mathrm{m}$ for medium and large spheroids respectively. The medium-sized spheroid diameter reached a plateau at six days then decreased in size. Large C6 spheroids reached a diameter plateau after five days but then remained stable. U251 spheroid diameter increased over the first three days, peaked at $146 \pm 3.9 \mu \mathrm{m}$ between 3-6 days and then decreased. C6 cell invasion continued at a maximal rate for 5-8 days and then decreased. Total invasion distance was $2752 \pm 87.7 \mu \mathrm{m}$ over 14 days and $2354 \pm 31.9 \mu \mathrm{m}$ over 12 days for medium and large C6 spheroids respectively. U251 cells continued to invade at a linear rate to a maximum distance of $812 \pm 50.5 \mu \mathrm{m}$ over 14 days.

Conclusion: Spheroids in three-dimensional collagen gels invade the gel by a combination of increasing spheroid growth and single cell invasion that model in vivo human tumor growth. Initial spheroid expansion is due to continuing cell surface mitosis and the eventual stabilization and/or regression in spheroid size is attributed to invasive cell loss. Therefore, invasion is best monitored, in this model, from the initial spheroid margin.

\section{NEUROMUSCULAR}

\section{P-039}

\section{Duchenne muscular dystrophy - parental perceptions}

J.E. Bothwell, J.M. Dooley, K.E. Gordon, A. MacAuley, P.C. Camfield, J. MacSween (Halifax, Nova Scotia)

Background: Quality of life and availability of services for DMD boys and their families is important.

Methods: Questionnaires were mailed to all families of boys with DMD attending our centre. Families gave an opinion on importance for services, health issues and quality of life issues "now" and "in the future".

Results: $31 / 35$ families (89\%) completed questionnaires. Services felt to be very important "now" were physiotherapy (77\%) and research into the genetics and treatment $(77 \%$, and
$84 \%$, respectively). Important health issues "now" were mobility and use of steroids (both 58\%). Comparing "now" and "in the future", parents identified increasing need for physiotherapy $(\mathrm{p}=0.05)$, occupational therapy $(\mathrm{p}=0.06)$, respirology $(\mathrm{p}=0.02)$, and health issues of loss of mobility $(\mathrm{p}<0.01)$ and assisted ventilation $(\mathrm{p}<0.01)$. Future quality of life concerns include increasing patient social isolation $(\mathrm{p}=0.01)$, anger $(\mathrm{p}=0.04)$ and depression ( $\mathrm{p}=0.03$ ). Families of older boys (beyond six years of diagnosis), recognized psychiatry as having an increasingly important role for their boys in the future.

Conclusions: Parents' values of services and quality of life issues for their sons with DMD have been described and are seen to change with time and time from diagnosis. This may have implications for services delivery within neuromuscular clinics.

\section{P-040}

An association between inclusion body myositis and systemic amyloidosis

J.M. Boulanger, J.B. Lamarche (Sherbrooke, Quebec), J.P. Bernier (Fleurimont, Quebec)

Background: B-amyloid is identified in muscle fibers of patients with inclusion body myositis (IBM). Systemic amyloidosis (SA) can lead to a myopathy in which amyloid material is deposited between muscle fibers.

Objective: To describe a case of an association, yet unreported, between IBM and SA.

Clinical presentation: A 71-year-old man progressively developed hoarseness of voice and muscle weakness. A muscle biopsy demonstrated typical findings of IBM on light and electronic microscopy. Individual myocytes didn't show any $\beta$ amyloid immunoreactivity but the reaction was strongly positive in the muscle's blood vessels and between muscle fibers. Later on, the patient presented signs of right sided heart failure and purpura. Aspiration of subcutaneous abdominal fat confirmed the diagnosis of SA. Bone marrow biopsy revealed an increased population of plasmocytes (9-10\%). Otherwise, we found no evidence of multiple myeloma. IVIG, melphalan and prednisone so far have not showed any benefit.

Conclusion: Amyloid deposits are noted in IBM and SA. We hypothesize that they share common pathogenic mechanisms. Oxidative stress, as suggested in the literature, could promote amyloid formation in susceptible individuals who overexpress abnormal proteins.

\section{P-041}

Neonatal respiratory status and outcome in children with congenital myotonic dystrophy

\section{Campbell, R. Sherlock, P. Jacob, M. Blayney (Ottawa,} Ontario)

Background: Myotonic dystrophy presenting in the neonate with respiratory failure and hypotonia is termed congenital myotonic dystrophy (CDM). Limited literature suggests that children with CDM ventilated longer than 30 days have uniformly high mortality. Withdrawal of life support is often recommended in such situations. Our experience supports an 
alternate hypothesis: children with $\mathrm{CDM}$ requiring prolonged ventilation ( $\geq 30$ days) have the same outcome as those who do not.

Methods: Retrospective chart review of children with CDM followed at the Children's Hospital of Eastern Ontario born between 1980-1997. Neonatal respiratory characteristics, mortality, life span and long-term medical and developmental outcomes were collected.

Results: Fourteen children were reviewed. Two died after withdrawal of ventilation shortly after 30 days of life in the absence of significant coincidental illness. The remaining children were divided into those initially ventilated less than 30 days (group 1, n=6) and thirty days or longer (group 2, n=4). Two children could not be classified. One death occurred in each group at 20 months and 10 months respectively. The oldest of the remaining children are 17 years and 13 years respectively. Total hospitalizations, language, motor and school scores at 1, 3 and 6 years were similar for the two groups.

Conclusions: Children with $\mathrm{CDM}$ requiring prolonged ventilation have similar mortality, life span, morbidity and developmental outcome as those children ventilated less than 30 days.

\section{P-042}

\section{Polytherapy approach for IVIG resistant chronic inflammatory demyelinating polyneuropathy}

\section{F.H. Jacques (Hull, Quebec)}

Background: On average $60-70 \%$ of patients with chronic inflammatory demyelinating polyneuropathy (CIDP) will respond to intravenous immunoglobulins (IVIG) therapy. In patients with severe rapidly progressive IVIG resistant CIDPthe treatment options are limited. The conventional approach of empirically trying and/or adding one drug at a time is slow and perhaps inappropriate in a disease such as rapidly progressive CIDP where time may be equated with axonal loss. Between November 1999 and March 2000, two patients with severe IVIG resistant CIDP were treated with a polytherapy approach. At the time of initiating therapy both were bedridden.

Methods: Treatment protocol consisted of two monthly courses of plasmapheresis, four monthly IV cyclophosphamide, prednisone and $\mathrm{S} / \mathrm{C}$ interferon alpha2a given together thrice a week. All treatment modalities were begun simultaneously. Prednisone taper was begun at the third month of therapy.

Results: Both patients obtained rapid complete functional recovery. Remission is sustained in both, using prednisone and interferon alpha2a. Electrodiagnostic studies done six months post initiation of therapy, has shown significant improvement in both. No significant side-effects were noted.

Conclusions: In these two patients with rapidly debilitating IVIG resistant CIDP the polytherapy approach was rapidly and highly effective. The protocol was well tolerated.

\section{P-043}

\section{Severe infantile facial diparesis in FSHD with $\mathbf{4 q 3 5}$ deletion}

N. Jetté, P.R. Bourque, J. Allanson (Ottawa, Ontario)

Background: In a 1971 paper, Rowland first reported on a Moebius-like syndrome of congenital facial diplegia in three children subsequently diagnosed with facioscapulohumeral muscular dystrophy (FSHD). The identification of a 4q35 deletion in 90-95\% of FSHD has greatly enhanced the awareness of phenotypic heterogeneity in this syndrome.

Methods: We studied two patients who were initially diagnosed with Moebius syndrome and later developed a typical FSHD phenotype. The chromosome 4q35 mutation was detected by hybridization with p13E-11 after digestion with EcoRl/Blnl.

Results: A 20-year-old woman had infantile-onset disabling facial diparesis. She only became aware of right scapular winging at age 15, although she subsequently developed abdominal, scapular and peroneal paresis. CK was mildly elevated at $265 \mu / \mathrm{L}$. The $4 \mathrm{q} 35$ fragment was $20 \mathrm{~kb}$ in size.

The second patient also had infantile-onset evident facial weakness and developed markedly progressive scapular and lower limb weakness starting at age 10 , requiring a motorized chair by age 15 . Her 4q35 fragment size was $15 \mathrm{~kb}$.

Conclusions: Marked infantile facial diparesis may precede the detection of appendicular weakness by more than a decade in some patients with FSHD. Testing for the 4q35 mutation should be considered in some cases of "Moebius" syndrome, particularly if horizontal gaze palsy or skeletal anomalies are absent.

\section{P-044}

\section{Acute peripheral and central neurotoxicity with metronidazole - a case report}

P. Jobin, J-P. Bouchard, D. Brunet, P.V. Gould (Quebec City, Quebec)

Background: Metronidazole is a nitroimidazole antibacterial and antiprotozoal agent. Neurotoxicity may occur. Since the original description by Ramsay in 1968, about 40 cases of polyneuropathy have been reported. Typically, it consists of a progressive axonal sensitive polyneuropathy. This syndrome develops after a total dose between 30-200g. The course of the polyneuropathy varies after drug discontinuation. The central toxicity is mainly cerebellar and rapidly reversible.

Case: A 80-year-old man, with refractory $C$. difficile colitis, was seen for a five-day history of dysarthria, distal paresthesia and ataxia. He had taken $2 \mathrm{~g}$ /day of metronidazole for 45 days. The physical examination revealed dysarthria, diffuse weakness (4/5), decreased reflex and ataxia. All sensory modalities were markedly decreased. The blood tests, CSF and brain MRI were unremarkable. The electrophysiologic evaluation and the sural biopsy confirmed a predominantly sensitive axonal polyneuropathy with involvement of large myelinated fibers. The metronidazole was discontinued and the patient improved significantly in the next few days. Unfortunately, one year later he still has a severe clinical and electrophysiological sensory deficit. 
Conclusion: This case illustrates the well-known presentation of metronidazole neurotoxicity but is also noteworthy for the acute evolution, the importance of the motor deficit and the combination of peripheral and central (dysarthria and ataxia) deficit.

\section{P-045}

\section{Sensory neurons and Schwann cells in pharmacological stimulation of nerve regeneration}

\section{Raginov, Yu. Chelyshev, T. Shagidullin (Kazan, Russia)}

Background: Success of nerve regeneration is determined by the ability of damaged neurons to survive after trauma. Neuronal survival depends in the efficacy of axonal elongation and retrograde transport in perikarion of neurotrophic factors, Schwann cells of the injured nerve in particular being the source of it.

Methods: Using the model of rat sciatic nerve transection and crush, we studied influence of pyrimidine derivative xymedon on efficacy of regeneration of myelinated axons, number and phenotype of surviving sensory neurons (growth-associated protein (GAP-43) and apoptosis antagonist Bcl-2 protein) and Schwann cells (S100, GAP-43 and proliferating cell nuclear antigen) on the 7 th, 15th, 30th, 60th and 90th day after nerve injury.

Results: Positive increase of the number of regenerating myelinated fibers in all stages after crush and in 60 and 90 days after nerve transection were observed after xymedon administration. The number of Schwann cells in distal parts of the nerve in the test group was positively higher than in the control in all the stages after transection and 15, 30 and 90 days after crush. On the 7 th day after nerve crush the number of proliferating and GAP-43+ Schwann cells on cross-section of the test group at the site of the operation was positively higher than in controls, by $82,9 \%$ and $29,9 \%$ respectively. On the 60 th and 90th day after nerve transection the amount of sensory neurons in DRG on the side of operation was higher in the test group than in the controls, by $22 \%$ and $26 \%$ respectively. Compared to controls, administration of xymedon at early stages after injury resulted in larger number of neurons expressing GAP-43, while at the late stages, xymedon caused decrease of GAP-43 neurons. Compared to controls, administration of xymedon at all stages after injury resulted in larger number of neurons expressing Bcl2.

Conclusions: Decreased sensory neuron post-traumatic death under xymedon, as well as increased tham antiapoptosis protein Bcl-2 expression may be attributable to the direct apoptosis inhibiting effect of medication and/or to the increased volume of the Schwann cell population in the injured segment and the increase in neurotrophic factors production, supporting neuronal survival.

\section{P-046}

\section{The effect of propentofylline on microglial/motor neuron interactions in vitro}

M.J. Strong, B.P. He (London, Ontario)

Background: Because microglial activation has been observed in association with degenerating motor neurons in amyotrophic lateral sclerosis (ALS), we determined whether motor neuron injury in vitro can be mediated by microglia, and the effect of the phosphodiesterase inhibitor propentofylline (PPF) on this process.

Methods: The supernatant from a serum-deprived motor neuron hybridoma cell line (NSC-34; Dr. Neil Cashman) was applied to microglia (BV-2 cell line; Dr. F. Blaisi). After 24 hours, the supernatant from the microglial cell line was applied to otherwise healthy NSC-34 cultures or a TNF $\alpha$ sensitive cell line (actinomycin treated L929 cells).

Results: BV-2 cells exposed to serum-deprived NSC-34 conditioned medium upregulated NO generation (DAF-2A fluorescence) and migration rates within 24 hours. Application of supernatant from these BV-2 cells to healthy NSC-34 cells resulted in increased NSC-34 cell NO generation. Microglia treatment with PPF suppressed this effect. Neither NSC-34 or L929 cells demonstrated increased cell death (MTT assay). Unconditioned BV-2 medium had no effect.

Conclusions: In vitro, microglial cells become activated in response to motor neuron injury and are capable of upregulating healthy motor neuron NO generation via unknown soluble factors. This process can be inhibited by treatment of microglia with PPF.

\section{P-047}

\section{Clinical epidemiology of amyotrophic lateral sclerosis} (ALS) in central and northeastern Quebec

\section{S.Verreault, F. Émond, F. Gosselin, J.-P. Bouchard (Quebec} City, Quebec)

Background: The main clinical features of ALS are well known and have been studied in many population groups. However, no such study was ever done in Quebec until now.

Methods: Between August 1, 1993 and October 31, 2000, 137 patients were diagnosed with ALS according to the "El Escorial" criteria and followed at the neuromuscular clinic in Quebec City. They all participated in a double-blind placebo-controlled clinical trial (ending December 31, 1994) and/or the early access program with Riluzole oral treatment. This cohort was analyzed retrospectively.

Results: A total of 77 men and 60 women (1.28/1.0 ratio) were included. There was no familial case. Ten patients were lost at follow-up. Age at onset for males was 55 years \pm 12.4 (range: 20-78) compared to $60 \pm 11.6$ (range: 33-78) for females. Moreover, the duration of the disease for deceased patients was 31 months \pm 15 for men compared to $39 \pm 19$ for women. Bulbar onset was noted in 40 cases $(29.3 \%)$. In the Greater Quebec area (population: 644,500), 13 patients were alive at the end of this study, for a prevalence rate of 2/100,000.

Conclusions: This cohort has many similar clinical 
characteristics compared to previous studies. However, we report a striking difference in age at onset and duration of disease between men and women. Further data on age groups, occupation and clusters will be presented.

\section{EPILEPSY}

\section{P-048}

\section{Threshold of afterdischarge in children with neuronal migration disorders}

S. Chitoku, H. Otsubo, A. Hunjan, J.T. Rutka, S. Weiss, O.C. Snead III (Toronto, Ontario)

Background: The purpose of this study was to investigate the factors related to threshold of afterdischarge (AD) in children with or without neuronal migration disorders (NMD).

Methods: The amperage threshold of primary motor cortex and $\mathrm{AD}$ in 23 pediatric epilepsy patients (aged 4-18 years) were examined during intracranial EEG recording. We divided the patients into two groups according to pathological and neuroimaging results: (A) 11 patients with NMD and (B) 12 patients with other disorders.

Results: The amperage thresholds of AD were higher (4$20 \mathrm{~mA}$; mean 11.6) in group A than those in group B (6-14mA, mean 10.1) under the age of 12 years. Older than 12 years old, amperage threshold of AD in group A became lower $(3-10 \mathrm{~mA}$ : mean 5.4) than those of group $B(8-12 \mathrm{~mA}$, mean 8.6). Amperage thresholds of $\mathrm{AD}$ were lower than those of primary motor function in both groups. Both amperage thresholds had an inverse relationship with age. Difference of amperage threshold between $\mathrm{AD}$ and motor function is smaller in group $\mathrm{A}$.

Conclusions: Important factors are patients' age, pathology, and motor and $\mathrm{AD}$ amperage thresholds to complete pediatric functional mapping safely. In adolescence, lower amperage threshold of AD suggests the hyperexcitability of epileptic brain in NMD.

\section{P-049}

\section{Barriers to work in persons with epilepsy}

\section{B. Clarke, R. Williams, A. Upton, C. Castellanos (Hamilton,} Ontario)

Background: Seizure control is seen to be the key obstacle to work in persons with epilepsy. Contributions of suffering, pain, perceptions of self-efficacy, and work beliefs have not been delineated.

Methods: A pilot study of 55 epileptic subjects, (22 females, mean age $41 \pm 10$ S.D.years) were given a validated questionnaire to assess the above attributes. $27 / 55$ subjects had a paid job, $57 \%$ were not on a disability pension, $30 \%$ had multiple disabilities, $30 \%$ had severe pain, none were seizure free.

Results: Work beliefs and self-efficacy were the best predictors of work $[\mathrm{F}(1,53)=80.31, \mathrm{p}=0.001]$. Total suffering scores correlated strongly with total self-efficacy scores at $\mathrm{r}=-$ $0.70, p=0.01$ as did total pain scores at $r=-0.62, p=0.01$. There were no significant differences between subjects who worked and those who did not and work beliefs. Total self-efficacy scores were higher for the work group. $60 \%$ of subjects believed their family did not want them to have a job, $47 \%$ said seizures would not affect job performance, but $50 \%$ were concerned about fear in co-workers if seizures occurred.

Conclusions: Negative perceptions of self-efficacy, suffering, poor work beliefs and pain are important barriers to work in epilepsy.

\section{P-050}

Atypical absence seizures in Long Evans hooded rats are refractory to the ketogenic diet

\section{Serbanescu, M.A. Cortez, M.A. Ryan, S. Cunnane, O.C. Snead III (Toronto, Ontario)}

Background: Human atypical absence seizures are usually refractory to the ketogenic diet (KD), therefore we set out to determine its degree of effectiveness in the AY-9944 model.

Methods: Thirty-four adult Long Evans hooded rats were implanted with epidural electrodes at postnatal day (PD) 40 after developmental AY-9944 (AY) treatment $(7.5 \mathrm{mg} / \mathrm{kg})$ or equivalent of saline from PD 2 to PD 20. One hour baseline, control diet $(\mathrm{CD})$ and $\mathrm{KD}$ posttreatment $\mathrm{EEG}$ recordings were performed at PD 40, 56 and 67, in male (M) female (F) controls (CC) and AY treated subjects for quantification of SWD duration as a measure of absence seizure occurrence. Animals were sacrificed for determination of plasma, liver and brain sterol levels.

Results: Recurrent atypical absence seizures were captured at baseline, $\mathrm{CD}$ and $\mathrm{KD}$ recordings. SWD (Mean $\pm \mathrm{SD}$ ), MCC (7.9 \pm 4$)$, FCC (45.8 \pm 3.25$), \quad M C-K D ~(31.2 \pm 28.1), \quad F C-K D$ (70.9 \pm 40.5$)$, MAY-CD (310.9 \pm 77.3$)$, FAY-CD (358.2 \pm 91.7$)$, MAY-KD (286.9 \pm 19$)$, FAY-KD (435 \pm 54$)$, ( $>0.357$ ANOVA). Body weight differences were not significantly different between test and controls ( $\mathrm{p}=0.445$, ANOVA).

Conclusions: Data indicate that chronic atypical absence seizures induced by cholesterol synthesis inhibition during development are resistant to the ketogenic diet.

\section{P-051}

Does the focality of complicated infantile febrile seizures predict the lateralization of intractable temporal lobe seizures in the adult?

\section{L.M. Korngut, J.P. Girvin (London, Ontario)}

Hypothesis: The presence of complicated febrile seizures in infancy constitutes a significant risk factor for the development of temporal lobe epilepsy in the adult.

Research question: Does the lateralization (focality) of complicated febrile seizures in infancy correlate with the lateralization of intractable complex partial seizures of temporal lobe origin (when such occur) in adults?

Methodology: Retrospective review of patients undergoing anterior temporal lobectomies for the treatment of intractable seizures of temporal lobe origin, operated upon at the University Campus of the London Health Sciences Center over the last 20 years, who have a history of complicated febrile seizures in 
whom the focality of such seizures can be clearly ascertained. Within this study, the exclusion criteria included previous head injuries, intracranial masses, CNS infections and Sturge-Weber disease. The inclusion criteria consisted of:

1) Occurrence of complicated febrile seizures between the ages of six months and five years;

2) The presence of lateralized intractable complex partial seizures of temporal lobe origin who were treated by anterior temporal lobectomy;

3) A significant improvement in the seizure disorder as a result of the anterior temporal lobectomies; and

4) The absence of exclusion criteria.

To-date 58 patients have been reviewed, 27 of whom were found to have had complicated febrile seizures in infancy. Twelve of the 27 patients with complicated febrile seizures had evidence of lateralization, or focality, and in 10 out of 12 the side of lateralization was clearly documented.

In all of the 10 patients in whom there was documented febrile seizure lateralization, there was positive correlation with the side of adult origin of the complex partial seizures. In all 10 patients temporal lobectomy resulted in significant improvement in the habitual seizure disorder.

Conclusion: The data to-date suggest clearly that recognition of the lateralized aspects of complicated febrile seizures in infancy leading to habitual seizures of temporal lobe origin would predict the side of the intractable epileptic foci in adults.

If the foregoing noted observations to-date hold up in the larger study of such patients, then there are widespread economic and practical implications. If the correlation turns out to be a $100 \%$, then taken to the extreme it would preclude the necessity for anything but a minimum of outpatient investigation in the adults in order to determine the lateralization of their foci. Further, the lack of requirement for the admission of such patients to epilepsy units would significantly decrease the cost of investigation of such patients.

\section{P-052}

\section{Linear growth and nutritional status of children with epilepsy on the ketogenic diet: a pilot study}

\section{$\underline{Y}$. Liu, S. Williams, R. Curtis (Toronto, Ontario),}

\section{Basualdo-Hammond (Edmonton, Alberta)}

Background: The ketogenic diet is one of the most effective therapies for intractable seizures. Potential growth and nutritional risks have been evaluated by limited studies. This study investigated the impact of the medium chain triglyceride and classic ketogenic diets on children's nutritional status.

Methods: Participants included 16 children on the classic and 14 on the MCT diet. All children had failed at least two anticonvulsant treatments. Weight, height, skinfold measurements and biochemical indexes were obtained at pre-diet and four months on the study. Paired-t tests were conducted.

Results: $83 \%$ of participants completed the study. Both groups had statistically significant height increases $(\mathrm{p}<0.05)$; post intakes of protein, vitamins (A, B1, B2, B6, C, D, E) and minerals (calcium, magnesium, iron, phosphorus, zinc) met or were close to recommended nutrient requirements; and all biochemical indexes (serum albumin, calcium, magnesium, zinc, vitamin $\mathrm{E}$, phosphate, ferritin) remained within the normal range. The classic diet group had significantly lower albumin and magnesium, higher ferritin and vitamin $\mathrm{E}$ at four months $(\mathrm{p}<0.05)$ and higher total cholesterol/HDL ratio associated with increased cardiac risk. The MCT group, in contrast, had a significantly lower cholesterol/HDL ratio $(\mathrm{p}<0.05)$ but no significant differences among other biochemical indexes.

Conclusions: This study demonstrated that the nutritional status and linear growth of children on the ketogenic diet could be maintained over a four month period.

\section{P-053}

Focal malformations of cortical development with balloon cells - clinicopathological correlates in paediatric epilepsy surgery patients

M.T. Mackay, L.E. Becker, H. Otsubo, O.C. Snead, J. Rutka, S.K. Weiss (Toronto, Ontario)

Background: Balloon cells, a pathognomonic feature of tuberous sclerosis (TS) are also seen in focal cortical dysplasia (FCD). We report clinical-radiological features in children whose focal cortical resections demonstrated balloon cells to determine if there was a difference between these subgroups.

Methods: Retrospective case review from a pathology database search (1990-2000). Classification as focal cortical dysplasia with balloon cells (FCDBC) or TS was based on clinical criteria.

Results: Forty-nine patients were identified with a pathological diagnosis of FCD or TS. Eighteen (37\%) had balloon cells on histology, seven had TS, 11 had FCDBC. All had partial motor seizures. Mean age at seizure onset and surgery were 17 and 86 months for FCDBC, and nine and 77 months respectively for TS patients. Three in each group had hemiparesis. MRI did not identify FCD in four FCDBC patients. Preoperative clinical diagnosis of TS was not made in all three children with solitary tubers. Eight (73\%) of 11 FCBC and two $(33 \%)$ of six TS patients are seizure free (mean follow-up duration 45 months).

Conclusions: Seizure onset is later and surgical outcome better in FCDBC patients. Balloon cells are commonly seen in FCD (26\% in our series) but their genetic relationship to TSC mutations is unclear.

\section{P-054}

Integration of pre-operative speech mapping with PET scanning for tumour and epilepsy surgery. Experience with 133 cases

\section{A. Olivier, D. Klein, B. Milner, W. Boling, A. Bastos (Montreal, Quebec)}

Background: Speech localization has always been of the utmost importance for resection of tumours and epileptogenic lesions in the dominant hemisphere. Traditionally, per-operative stimulation and mapping have been used. The advent of PET and later fMRI and the possibility of integrating pre-operative activation studies within the image guidance surgery have significantly improved our ability to resect lesions in eloquent areas. 
Method: 133 patients (142 scans) with various intrinsic glial tumours (41) and other epileptogenic lesions (vascular, developmental) involving eloquent areas of the dominant hemisphere have undergone pre-operative PET activation of speech centers using $\left[{ }^{15} \mathrm{O}\right] \mathrm{H}_{2} \mathrm{O}$. Typically, all patients were scanned twice in each of four conditions: a silent resting baseline condition, a condition of passive listening to words, a condition of repetition of heard words and a condition in which synonyms are provided for each word heard. Co-registration of PET and MR images was performed using a cross-correlation algorithm and integrated in the operative $3 \mathrm{D}$ and $2 \mathrm{D}$ image guidance system (Scout SNN).

Results: Zones of activation for the different tasks correspond rather faithfully to the classical speech areas as determined by per-operative stimulation with increased precision in the spatial distribution. In most instances, speech dominance can be established. As a general rule, speech centers are not invaded by low-grade tumours but rather displaced. Long-standing lesions give rise to spatial functional re-organization. Zones of preoperative speech activation can be retrieved during surgery through image guidance and avoided during resection. Several patients presented transient dysphasia postoperatively but none developed a permanent deficit.

Conclusion: Pre-operative speech mapping with PET scanning provides crucial information concerning the zone of activation and its relationship to epileptogenic lesions including brain tumours. As a general rule, speech centers are not invaded but rather displaced by low grade brain tumours. Speech mapping can be integrated in the surgical image guidance system to minimize speech impairment associated with resections in the dominant hemisphere.

\section{P-055}

\section{Quality of life after surgery for epilepsy: a prospective study based on a 10-item questionnaire (QOLIE-10)}

André Olivier, N. Poulin, P. A. Baillargeon (Montreal, Quebec)

Background: Freedom from seizures is presumed to improve quality of life. Therefore, there is a need for more sensitive outcome assessment, including psychosocial functioning and quality of life. This prospective study was conducted to evaluate a brief questionnaire which screened aspects of health-related quality of life for patients suffering from temporal lobe epilepsy before and after surgery, to better define the impact of surgery on quality of life.

Method: 50 consecutive patients having undergone surgery for temporal lobe epilepsy (selective amygdalo-hippocampectomy and anterior temporal resections) were asked to fill out the questionnaire on quality of life (QOLIE) before surgery and two years after. The QOLIE-10 can be completed by a patient in several minutes and reviewed rapidly by the physician.

Results: The 10-item questionnaire on quality of life (QOLIE) covers general and epilepsy-specific domains, grouped into three areas: epilepsy effects (memory, physical effects, and mental effects of medication); mental health (energy, depression, overall quality of life), and; role functioning (seizure worry, work, driving, social limits). Overall, the two year postoperative evaluation shows a marked improvement for the three areas as judged by the patient.

Conclusions: Our preliminary results indicate that this screening tool not only provides useful information for initial assessment and follow-up of problem areas not commonly evaluated during routine clinical visits but can be also a good indicator of the improvement of quality of life following surgery.

\section{P-056}

\section{The callosal grid: a proportional reference system for brain imaging}

André Olivier, R. Lehman, L. Soualmi, W. Boling (Montreal, Quebec)

Background: With the explosion of modern imaging techniques based on magnetic resonance, there is a need for anatomical reference systems such as the commissural one proposed by Talairach that can be used by all workers in this field. The purpose of this presentation is to explain the rationale for the use of the corpus callosum as a basic landmark for brain imaging, to illustrate how the callosal grid is constructed and to provide examples of its usefulness as an imaging, anatomical and surgical tool. Although these planes and compartments are specific for each individual, they are proportional and can be used to "average" series of brain.

Methods: MRI, cadaver brains, atlases and imaging software have been used to construct and display the callosal grid. Basic measurement of structures such as the length of the brain, length of the corpus callosum, length of pre and post callosal segments have been obtained in 100 patients.

Results: The proportional callosal grid is essentially made up of a series of anatomic planes derived from the corpus callosum which delineate various compartments. These are the horizontal plane (HP) which passes by the under surface of the genu and splenium and the anterior, mid and posterior callosal planes which are perpendicular to HP and pass by the corresponding segments of the corpus callosum.

Conclusion: The callosal grid is a simple reference method for brain imaging which can be used for localisation of structures, functional areas, lesions and zones of atrophy within various compartments. Examples will be provided of its use in the study of the topographic and functional anatomy of the brain and as a tool for diagnosis of atrophic lesions and surgical planning.

\section{P-057}

\section{Hz vertex dominant EEG rhythm: a rare ictal feature of temporal lobe epilepsy}

\section{R.M. Sadler, S. Rahey (Halifax, Nova Scotia)}

Background: A 45-year-old male with partial complex seizures was evaluated for epilepsy surgery. Seizures were typical of temporal lobe epilepsy (TLE) and interictal EEG spikes were $100 \%$ left temporal. MRI revealed a medial left temporal lesion (likely a ganglioglioma). Ictal scalp EEG demonstrated a $5 \mathrm{~Hz}$ rhythm maximally expressed at the vertex. This unusual ictal rhythm was recently described (in a single 
case) as a manifestation of temporal lobe seizures confined to mesial-basal cortex (Pacia, Epilepsia 1997) and designated a "Type 1B seizure". We conducted a retrospective study to determine the prevalence of Type 1B seizures in TLE.

Methods: All EEG-telemetry reports obtained since the inception of digital EEG (February 1996) to December 2000 were hand searched to identify Type 1B ictal rhythms.

Results: Among 79 telemetry patients, 56 patients with partial onset epilepsy had 291 scalp recorded seizures (43 patients with TLE and 200 seizures; 13 patients with extratemporal epilepsy and 91 seizures). Only one patient had a Type 1B pattern; this occurred in all seizures $(\mathrm{N}=5)$.

Conclusion: Type 1B seizures are rare but electroencephalographers should be aware of this potentially confusing ictal rhythm. The postresection outcome of this patient will be presented.

\section{P-058}

\section{An assessment of neurotoxic effects of colloidal silver}

B. Young, X. Liu, S. Leung, M. Sharpe (London, Ontario)

Background: Alternative medicines have not been adequately tested for potential neurotoxicity.

Case Report: A 71-year-old man with prostatic carcinoma drank a homemade suspension of colloidal silver (Ag) daily for 4 months and later developed myoclonic status epilepticus in coma. No other cause was found. Although bilaterally synchronous positive spikes were seen on the initial EEG, other EEGs did not show spikes during axial myoclonus.

Investigations: Plasma $\mathrm{Ag}$ was $40 \quad(\mathrm{n}<2.3) \mathrm{nmol} / \mathrm{L}$, erythrocyte Ag was $20(\mathrm{n}<10) \mathrm{nmol} / \mathrm{L}$ and $\mathrm{CSF}$ Ag was 2 nmol/L. Despite repeated plasma exchanges to lower the Ag, the myoclonus and coma persisted. In an in vitro experiment involving hippocampal slices of rat brain, field potentials in CA1 cell layer were recorded in response to Schaffer collateral stimulation before and after perfusion of colloidal silver at various concentrations. No changes of field potentials were found to indicate alteration of synaptic transmission or spike generation. Also, no paroxysmal activity, evoked or spontaneous, was noted.

Conclusion: Despite impressive clinical features, we were unable to prove a neurotoxic effect for colloidal Ag.

\section{P-059}

\section{Continuous EEG monitoring vs standard care in detection of epileptiform activity in comatose ICU patients: a randomized, controlled study}

\section{B. Young, G.S. Doig (London, Ontario)}

Background: Continuous EEG monitoring (CEEG) is a promising technology in intensive care unit (ICU) neurology. Epileptiform activity (seizures or spikes) is generally viewed as worthy of detection. CEEG has not been previously subjected to a randomized controlled study in various comatose patients in a general ICU.

Methods: Prospective, randomized controlled study. Patients were randomized to have CEEG (24-48 hours) or standard care
(SC). Inclusion criteria: patient in noniatrogenic coma in ICU, age $>16$ years. Exclusion criteria: brain death or hopeless prognosis, patients in coma from cardiac arrest.

Results: Of patients with acute structural brain lesions (ASBL) 31 had CEEG and 21 had standard care. Of patients with metabolic disorders (M) 24 had CEEG and 13 had standard care. Patient demographics were similar in compared groups. In patients with ASBL, epileptiform activity (EA) was found in 10 (32\%) patients with CEEG but in only two (9.5\%) with SC $(\mathrm{p}<0.05)$. In $\mathrm{M}$ patients, EAwas found in only two (8\%) CEEG patients and $0(0 \%)$ SC patients $(\mathrm{p}=0.4)$.

Conclusions: CEEG monitoring of patients with ASBLs is superior to SC in detecting EA; the yield of EA by CEEG monitoring in $\mathrm{M}$ patients is very low.

\section{P-060}

The effect of topiramate on various seizure types within the Lennox-Gastaut syndrome in children and adults

\section{B. Young, Z. Hutchinson, S. Levin (London, Ontario)}

Background: Lennox-Gastaut syndrome (LGS) is a severe generalized seizure disorder beginning in childhood. Our study examines the effectiveness and side effect profile of topiramate in our adult and pediatric LGS outpatients.

Methods: Retrospective review. Inclusion criteria: age 2-65 years, topiramate for $\geq$ six months, mental subnormality, generalized seizures, EEG generalized sharp and slow wave complexes. Exclusion criteria: insufficient information; progressive neurological disorder; noncompliance; other neurological, psychiatric or systemic disorder.

Results: 14 males and four females: age range 4-47 years, mean 20 years; nine patients $\geq 16$ years. 12 had multiple seizure types. 17 with generalized convulsive seizures: three $(18 \%)$ became seizure free; six $(35 \%)$ had $>50 \%$ reduction; two $(12 \%)$ showed $>25 \%$ reduction in seizures; six $(35 \%)$ no benefit. 11 with drop attacks: two (18\%) were seizure-free; three (27\%) had $>50 \%$ reduction; six $(55 \%)$ showed no benefit. Four with atypical absence seizures: one $(25 \%)$ became seizure-free; one $(25 \%)$ had $>50 \%$ reduction; two $(50 \%)$ showed no benefit. Both $>16$ and $\leq 16$ year groups benefited. Topiramate was withdrawn in five $(28 \%)$ patients because of side effects and one $(5 \%)$ for lack of effectiveness.

Conclusions: Topiramate is associated with significant improvement seizure frequency in all major seizure types in LGS children and adults. Significant side effects requiring withdrawal occur in $28 \%$ of patients. 


\section{GENERAL NEUROLOGY}

P-061

\section{Measurement of serum methylmalonic acid to diagnose cobalamin deficiency in patients with posterior column dysfunction and normal serum vitamin $B_{12}$ levels}

M.B. Cossoy, T.J. Benstead, R.M. Sadler (Halifax, Nova

Scotia)

Background: Vitamin $\mathrm{B}_{12}$ (cobalamin) deficiency is a wellknown cause of neurological impairment, usually starting with symptoms of posterior column disease. Serum $B_{12}$ levels are usually, but not always, below normal in these patients. Cobalamin is an essential cofactor for metabolism of methylmalonic acid (MMA) and homocysteine (Hcy). Elevated serum MMAand Hcy levels may be a more sensitive indicator of cobalamin deficiency than serum $\mathrm{B}_{12}$.

Methods: Three patients with neurological symptoms consistent with cobalamin deficiency and normal serum vitamin $\mathrm{B}_{12}$ levels had serum MMA and Hcy levels and magnetic resonance imaging (MRI) of the brain and, in two patients, spinal cord.

Results: All three patients were men in their 60s. Two presented with subacute combined degeneration, while one had posterior column dysfunction alone. All had normal MRI of the brain. One had patchy hyperintensity affecting the mid- to posterior cervical cord on MRI. Serum B 12 levels $(225,177$, and $159 \mathrm{pmol} / \mathrm{L})$ were all normal (156-672 pmol/L). MMA levels $(127,025,461$, and $520 \mathrm{nmol} / \mathrm{L}$ ) were all elevated (normal: 73$271 \mathrm{nmol} / \mathrm{L}$ ), and one patient had an elevated serum Hcy level (168.1, 10.8, and 11.1 $\mu \mathrm{mol} / \mathrm{L}$; normal: 5.1-13.9 $\mu \mathrm{mol} / \mathrm{L})$. All three clinically improved on vitamin $\mathrm{B}_{12}$ replacement therapy, and one had documented normalization of serum MMA.

Conclusions: Patients may develop neurological complications of cobalamin deficiency with normal serum $\mathrm{B}_{12}$ levels. Serum MMA is useful for diagnosis in patients with normal serum $\mathrm{B}_{12}$ levels, but clinical features of cobalamin deficiency. Serum Hcy was not reliably elevated in these cases.

\section{P-062}

\section{The pinky finger in neurological diagnosis}

\section{A. Ogunyemi, D. Murphy (St. John's, Newfoundland)}

Background: Encounter with patients having somatoform disorder is not uncommon in medical, neurological or psychiatric practice. Difficulties sometimes arise in differentiating between these psychopathologic conditions and diseases with pathophysiological explanations.

In this report, we describe a simple clinical sign which can assist in distinguishing between patients with bona fide neurological diseases and those having psychogenic paresis or weakness involving the upper limb.

Method: Reflex movement of the pinky finger can be elicited in the following manner:

(i) the examiner instructs the patient to extend and abduct the

fingers of one hand; (ii) the patient's three fingers (2nd, 3rd, and 4th) are flexed at the metacarpophalangeal (MCP) joints while the patient resists the finger flexion;

(iii)the examiner observes the direction of movement of the pinky finger during the resistance to flexion of the $2 \mathrm{nd}, 3 \mathrm{rd}$, and 4th fingers.

Results: Normal subjects and all the patients with organic monoparesis, hemiparesis or quadriparesis caused by cerebral infarction, multiple sclerosis, or Guillain-Barre syndrome exhibited hyperextension of the pinky finger at the MCP joint (pinky finger phenomenon). In contrast, those patients with pseudodementia (depression); psychogenic hemiparesis and litigation-related, whiplash-induced upper limb monoparesis showed flexion of the pinky finger at the MCP joint during the resisted finger flexion (pinky finger sign).

Conclusion: The pinky finger phenomenon is a physiological, reflex movement while the pinky finger sign has a psychopathologic basis. Observation of the pinky finger movements as described, can assist at the bedside, in distinguishing between organic and psychogenic upper limb paresis.

\section{P-063}

An unusual presentation of acute disseminated encephalomyelitis (ADEM) following Mycoplasma pneumoniae infection

\section{A. Rajput, V. Chow, J. Donat (Saskatoon, Saskatchewan)}

History: A 22-year-old female presented with lower extremity paralysis, pain and areflexia. One week prior to admission she was febrile with a sore throat.

Physical Examination: 0/5 strength and areflexia of the lower extremities with mute plantars. Position and vibration were impaired in the legs with dysesthesia for pinprick. No sensory level was found. Her legs were extremely painful to passive movement. There was mild distal upper extremity weakness with preserved reflexes and normal sensation.

Investigations, course: CSF protein was elevated with mild lymphocytic pleocytosis. Initial blood work was unremarkable. She was diagnosed clinically with atypical Guillain-Barré and started on IVIG. Cefotaxime and acyclovir were added after she became febrile. Nerve conduction studies (NCVs) were normal. Intravenous methylprednisolone was added. MRI revealed diffuse expansion of the central cord from C3 to the conus with symmetrically abnormal $\mathrm{T} 2$ hyperintensity of the basal ganglia. No enhancement was noted. Three weeks after admission she had normal strength in the upper limbs and some movement of the lower extremities; NCV were essentially normal, but needle exam showed mild denervation. Serology was positive for Mycoplasma pneumoniae. We will present clinical, radiological and electrophysiological follow-up of this unusual presentation of ADEM. 


\section{P-064}

Are triptans truly contraindicated in patients with migraine and prolonged aura or migraine and stroke?

\section{Maher Saqqur (Edmonton, Alberta)}

The triptans have become the gold standard in the management of acute migraine. Their mechanisms of action include vasoconstriction $\left(5 \mathrm{HT}_{1 \mathrm{~b}}\right.$ receptors) and inhibition of neuronal conduction via the trigeminal nerve (5HT $1 \mathrm{~d}$ receptors). The vasoconstriction is not limited to the cerebral circulation but may also affect the coronary arteries. The use of such medications is therefore counter-indicated in patients with severe hypertension and ischemic heart disease. There is also a widespread belief that the drugs may increase the risk of stroke and their use is not recommended in patients who have migraine with prolonged aura, familial hemiplegics migraine or other conditions that may predispose to stroke. This recommendation has no clear scientific basis. There are now increasing reports of patients who have had a stroke or migraine with prolonged aura who did not develop any side effects or complications from such therapy. In this report, we describe three patients with conditions as described above, who took triptans with no ill effects.

The first patient is a 43-year-old female who presented with a history of classical headaches. During one headache, she took 15 tablets of sumatriptan with no relief. Subsequently when examined, she showed a left Horner's syndrome and investigation showed a left carotid dissection. The patient was anticoagulated and symptoms gradually improved. There appeared to be no side effects from the use of the large number of sumatriptans during the 2-3 days prior to diagnosis.

The second patient, a 48-year-old white male, had a history of familial hemiplegics migraine. He had been prescribed sumatriptan for many years, had no complications and treatment was quite effective in treating his migraine.

The third patient, a 41-year-old female, had a history of typical basilar artery migraine. Over the years, she had been taking an increasing amount of sumatriptan, zolmitriptan and more recently, rizatriptan for headache relief and did not notice any complications.

Patients with familiar hemiplegic migraine may have an alteration in the calcium channels. Patients with migraine and prolonged aura, in all likelihood, have a neuronal basis for their focal symptoms. Similarly, in patients who present with a stroke, by far the majority do not have symptoms related to vasoconstriction. Patients with migraine and prolonged aura or cerebral infarction from noncardiac sources may therefore not be at an increased risk for a complication of triptan use as has been suggested in the literature. A registry of such patients would be helpful in clarifying the risk of stroke or other complications with the prolonged use of triptans.

\section{P-065}

The vertical vestibulo-ocular reflex, and its interaction with vision during active head motion: effects of aging

James A. Sharpe, Ji Soo Kim (Toronto, Ontario)

Background: The vertical vestibulo-ocular reflex (VOR), and its interactions with vision during active head motion, and the effects of aging on these movements had not been investigated.

Methods: We measured smooth pursuit, combined eye-head tracking, the VOR, and its visual enhancement and cancellation during active head motion in pitch using a magnetic search coil technique in 21 younger (age <65) and 10 elderly (age $\geq 65$ ) subjects. With the head immobile, subjects pursued a target moving sinusoidally with a frequency range of 0.125 to $2.0 \mathrm{~Hz}$, and with peak target accelerations (PTAs) ranging from 12 to $789 \% \mathrm{~s}^{2}$. Combined eye-head tracking, the VOR in darkness, and its visual enhancement during fixation of an earth-fixed target were measured during active sinusoidal head motion with a peak-to-peak amplitude of $20^{\circ}$ at frequencies of $0.25,0.5,1.0$ and $2.0 \mathrm{~Hz}$. The efficacy of VOR cancellation was determined from VOR gains during combined eye-head tracking.

Results: The gain of smooth pursuit declined with increasing target frequency and peak target acceleration, having a marked decrease at accelerations over $99 \% \mathrm{~s}^{2}$. Combined eye-head tracking gain and VOR cancellation also decreased with increasing target frequency. VOR gain in darkness did not vary with the frequency of active head motion. Visually enhanced VOR (VVOR) gains declined significantly during head motion over $1.0 \mathrm{~Hz}$. Vertical smooth pursuit and combined eye-head pursuit showed directional asymmetry; downward gains were lower than upward, especially at high target accelerations. VOR cancellation was also less effective during downward head-free tracking at 0.5 and $1.0 \mathrm{~Hz}$. However, VOR and VVOR gains were symmetrical in both directions. In the elderly, smooth pursuit, and combined eye-head tracking gains and the efficacy of cancellation of the VOR were significantly lower than in younger subjects. VOR and VVOR gain did not change with aging, except for reduced gain of the downward VOR at low frequency $(0.25 \mathrm{~Hz})$.

Conclusions: Lowered gains at high target accelerations, directional asymmetry with lower downward gain, and senescent degradation are features common to vertical smooth pursuit, combined eye-head pursuit, and VOR cancellation. The upward and downward VOR and VVOR are symmetrical and vary little with advancing age.

\section{P-066}

The heat shock protein alpha-B-crystallin as possible autoantigen in multiple sclerosis: humoral responses in MS patients and controls

\section{Ursell, B.L. Banwell, M. Hohol, M.A. Moscarello (Toronto, Ontario).}

Background: The small heat shock protein alpha-B-crystallin is an immunodominant $\mathrm{T}$ cell antigen in myelin isolated from multiple sclerosis (MS) patients, where its expression is significantly upregulated. Viral infection of $\mathrm{B}$ cells in vitro results in HLA-DR restricted presentation of alpha-B-crystallin to proinflammatory $\mathrm{T}$ cells with subsequent activation, suggesting a possible link between peripheral infection and cellular immune responses towards CNS myelin. In contrast, humoral responses to alpha-B-crystallin have not been well described.

Methods: Serum samples from 66 MS patients, eight patients 
with other neurological diseases (OND), and 51 healthy controls were tested for the presence of antibodies to alpha-B-crystallin using enzyme-linked immunosorbant assays (ELISA) and Western immunoblotting. Antibody titres were also determined in cerebrospinal fluid (CSF) from 19 MS patients and 12 patients with OND.

Results: Antibodies could be detected in $46 \%$ of serum samples from MS patients compared to $35 \%$ of healthy controls. In CSF, antibodies were present in $17 \%$ of MS patients and $33 \%$ of OND patients. Antibody reactivity was stronger in MS patients than in controls.

Conclusions: The presence of antibodies in healthy individuals suggests that immune responses to alpha-B-crystallin may not be pathogenic in MS. Alternatively, normal peripheral immune responses may become pathogenic when exposed to increased levels of strongly immunogenic antigens in target tissue. While unlikely to be causative, such responses may serve to strengthen and sustain the immune response in MS patients.

\section{P-067}

\section{Churg-Strauss syndrome: similar treatment for different severity?}

G. Vorobeychik, D. LaCaille, A. J. Stoessl, M. Knazzen, M. Mezei (Vancouver, British Columbia)

Objective: To describe two cases of Churg-Strauss syndrome (CSS) and review literature with respect to treatment options.

Background: CSS classically results in late onset severe asthma, eosinophilia, and vasculitis. Treatment regimes and responses are variable without well-defined guidelines.

Design/Methods: Case report of two cases and review of literature.

Results: Patient 1 was a 59-year old male with progressive respiratory distress, malaise, weight loss, and (+) pANCA. Patient 2 was a 35-year old systemically well female with granulomatous vasculitis with marked eosinophilia on skin biopsy. Both patients had mild asthma, severe mononeuritis multiplex, eosinophilia, and negative sural nerve biopsy. Both patients achieved remission of vasculitis with partial neurological recovery after treatment with combination of oral prednisone and cyclophosphamide, preceded in the patient 2 with IV methylprednisolone.

The literature review revealed poor prognosis with severe neurological involvement and controversy about early use of cyclophosphamide and IVmethylpredisolone. These cases raised the following questions (i) use of cyclophosphamide in mononeuritis multiplex alone (as severe organ damage); (ii) need of nerve biopsy in classical clinical presentation to guide management.

Conclusions: Early use of cyclophosphamide and IV methylprednisolone in two cases of CSS with mononeuritis multiplex lead to excellent clinical response.

\section{MOVEMENT DISORDERS}

\section{P-068}

\section{Improved xenograft survival with combined local and systemic immunosuppression}

A.Y. Alemdar, K.A. Baker, D. Sadi, V.C. McAlister, I. Mendez (Halifax, Nova Scotia)

Background: Cyclosporine-A (CsA), the most widely used immunosuppressant in neural transplantation, has toxic systemic side effects. Tacrolimus (FK506), a more potent immunosuppressant, can be encapsulated in liposomes (LTAC) and delivered locally, reducing the possibility of systemic side effects. In this study we investigated the ability of LTAC to promote graft survival when administered systemically, locally, or in combination in hemiparkinsonian rats implanted with mouse xenografts.

Methods: Rats with unilateral 6-hydroxydopamine lesions were transplanted with 800,000 fetal mouse dopaminergic cells and were randomly divided into four groups. Group 1 was not immunosuppressed; Group 2 received daily systemic injections of LTAC; Group 3 received LTAC within the cell suspension; and Group 4 received local LTAC in the cell suspension along with daily injections of LTAC. Transplanted rats were assessed for rotational behaviour post-transplantation, and tyrosine hydroxylase (TH) immunohistochemistry was used to assess graft survival.

Results: A significant reduction in rotational scores was observed only in animals receiving the combination of local and systemic LTAC, correlating with a significantly greater survival of TH immunoreactive cells in those animals.

Conclusion: A combination of local and systemic immunosuppression may be a superior immunosuppressive strategy to optimize graft survival and decrease systemic side effects. This strategy may have important implications for clinical neural transplantation.

\section{P-069}

\section{Survey of sialorrhea in parkinsonian patients in southwestern Ontario}

H.C. Hyson, A. Johnson, S. Wiebe, M.S. Jog (London, Ontario)

Background: While sialorrhea is a well-recognized symptom of parkinsonism, few studies have been conducted to assess the impact of sialorrhea on this patient population. To this end, a survey was designed to document the incidence of sialorrhea, its severity, and the attitudes towards the medical treatment of this symptom in patients suffering from parkinsonism in southwestern Ontario.

Methods: A survey consisting of 13 questions regarding sialorrhea prevalence and patient attitudes toward this symptom was prepared and distributed to the parkinsonian patients of a movement disorders practice in London, ON. Information obtained from this survey is to be used in planning future studies of the medical treatment of sialorrhea. 
Results: A total of 374 surveys were distributed, with 71 surveys having been returned (19\%). A total of $46.5 \%$ of respondents felt that sialorrhea was a problem. Of this group, $17 \%$ of patients felt their sialorrhea required treatment, though only one respondent felt it was the worst symptom of their disease. $18.8 \%$ of symptomatic respondents felt that their sialorrhea was socially disabling. Of symptomatic patients, only $9.4 \%$ had used any treatment in the past, even though $62.5 \%$ of patients were interested in treating this symptom with medication.

Conclusion: Results from this survey show that sialorrhea is a common symptom among parkinsonian patients, and that symptomatic treatment is desired by this group of individuals.

\section{P-070}

\section{A case of cortical venous thrombosis presenting as reversible parkinsonism}

M. Jenkins, N. Nabil Hussain, D. Lee, M. Jog (London, Ontario)

Background: We present a case of cortical venous thrombosis with reversible clinical and radiological parkinsonism, a distinctly uncommon presentation. Reversible structural causes of parkinsonism that show dramatic radiological changes and clinical signs of profound parkinsonism and yet completely reverse, are highly uncommon.

Methods: In our case, the patient developed an akinetic-rigid state associated with cortical venous thrombosis. MRI scans demonstrated signal abnormality on T2 and diffusion weighted imaging. Her clinical symptoms and radiological findings reversed with heparin treatment.

Results: Prior cases of such clinical and radiological reversibility are reviewed. Hypoxia, compression and infection are found to be the most common underlying etiologies. Reversibility of diffusion weighted MRI abnormalities is discussed. Pathophysiological mechanisms suggested are metabolic dysfunction including mitochondrial dysfunction; immediate early gene response; toxic injury including oxidative stress, glial activation, and inflammatory responses; and direct mechanical disruption. Utilizing the possible underlying mechanisms, a unifying hypothesis for the unique features seen in our case is presented.

Conclusions: Our experience and the experience of other authors demonstrate that structurally-induced parkinsonism can be a clinically and radiologically reversible phenomena. Cases such as ours provide interesting insights regarding the current understanding of possible pathogenesis of Parkinson's disease.

\section{P-071}

\section{Hemiparkinsonism-hemiatrophy syndrome treated with subthalamic nucleus stimulation}

\section{Jenkins, D. Mendonca, A. Parrent, M. Jog (London, Ontario)}

Background: We report a case of hemiparkinsonismhemiatrophy (HPHA), a unique form of secondary parkinsonism distinct from Idiopathic Parkinson's disease treated by sub- thalamic nucleus stimulation. In the original description by Klawans, the syndrome is characterized by somatic hemiatrophy and ipsilateral parkinsonism, dystonia, corticospinal dysfunction. Other features included early age of onset, slow disease progression, and poor response to levodopa.

Methods: Clinical and radiographic features of our case are presented. The clinical features of our case are in keeping with Klawans'original description. Similarly, in our patient, response to medical treatment was poor. Surgical implantation of a subthalamic (STN) stimulator dramatically improved her symptoms. There are no reported cases of use of STN stimulator in HPHA.

Results: The several unique presentations of HPHA are reviewed. Importantly, similarities and discrepancies from Klawans' original description are highlighted paying particular attention to clinical characteristics, neuroimaging, response treatment and pathogenesis. We propose that HPHAis a result of metabolic dysfunction of the striatum related to perinatal injury. MRI and PET scan findings, poor response to treatment, and somatic hemiatrophy would support this hypothesis.

Conclusions: Awareness of this unique syndrome is important for diagnostic, treatment and prognostic considerations.

\section{P-072}

\section{Clinical characteristics of parkinsonian patients with basal ganglia calcification on CT}

\section{A. Rajput (Saskatoon, Saskatchewan)}

Background: Basal ganglia calcification has been reported in up to $2 \%$ of all head CTs and is associated with a number of conditions including parkinsonism (PS). The clinical significance of basal ganglia calcification, however, is uncertain.

Methods: 17 patients $(\mathrm{F}=11, \mathrm{M}=6)$ with $\mathrm{PS}$ and basal ganglia calcification on head $\mathrm{CT}$ were identified from the medical records.

Results: Initial symptoms were: upper extremity tremor $(n=6)$, gait or lower extremity difficulty $(n=3)$, bradykinesia $(n=4)$, sleep difficulty $(n=1)$, memory impairment $(n=1)$ and unknown $(n=2)$. Mean onset age was 65.7 years. Only $2 / 16$ had no improvement with levodopa, with 11/16 having definite and $3 / 16$ having questionable improvement. Motor fluctuations were documented in 7/16. Final clinical diagnoses were idiopathic Parkinson's disease (IPD) $(n=8)$, IPD with dementia $(n=3)$, dementia + PS $(n=2)$, atypical PS with possible striatonigral degeneration $(n=1)$, progressive supranuclear palsy $(n=1)$, PS secondary to carbon monoxide poisoning $(n=1)$, and Fahr's disease $(n=1)$. Autopsy was obtained in four cases and pathological diagnoses were IPD $(n=2)$, IPD + diffuse Lewy body disease (DLBD) $(\mathrm{n}=1)$, and IPD + DLBD + Alzheimer's disease $(\mathrm{n}=1)$.

Conclusions: Parkinsonian patients with basal ganglia calcifications do not appear to have a significantly different clinical profile from the general parkinsonian population. 


\section{NEUROPHYSIOLOGY}

P-073

\section{The impact of ulnar nerve compression on hand function and on individuals in different occupations}

K.M. Chan, S. Wyldman, L. Satkunam, G.L.W. Lobay

(Edmonton, Alberta)

Background: Ulnar nerve compression is common but its relative impact on individuals in different occupations is unclear. The objective of this study is to examine the influence of ulnar nerve compression on hand function and to compare its impact on laborers with those engaged in sedentary occupations.

Methods: A total of five patients were studied. These included three laborers, each had a severe conduction block of the ulnar nerve at the elbow but without any sign of marked axonal loss. An initial assessment of their hand function at the time of diagnosis was done by using a battery of tests. These included monofilament test, Jebsen-Taylor Hand Function Tests, Moberg Pickup Test and Baltimore Tool Evaluation. For comparison, detailed motor, sensory examination and electrophysiologic studies were also done on two other individuals, one a housewife and the other an office administrator.

Results: In all three laborers, marked sensory deficit was present in the ulnar nerve territory. While none of them had any significant problem with fine manipulation and object recognition, their grip and pinch strength were markedly reduced. This posed major problems in their jobs that required heavy repetitive hand use. Two subjects subsequently underwent surgical decompression of the ulnar nerve. The conduction block completely resolved following surgery accompanied by a full functional recovery. The conduction block in the third patient resolved spontaneously which was also accompanied by a marked functional improvement. In contrast, the two sedentary patients, both had ulnar nerve compression at the elbow of similar severity, declined surgery as it did not cause any major difficulty in their work.

Conclusions: These cases illustrate the importance of the ulnar nerve in determining the grip strength that is particularly relevant to those engaged in heavy physical labor.

\section{P-074}

\section{Psychogenic paraplegia in a patient with normal electrophysiologic finding}

\section{S.S. Haghighi, S.H. Meyer (San Diego, California, USA)}

Background: Conversion disorder with motor system symptoms or deficit is a subtype which includes symptoms of impaired motor coordination or balance, paraplegia, muscle weakness, difficulty in swallowing, and urinary retention. A case report of psychogenic paraplegia was diagnosed using median $(\mathrm{MN})$ and posterior tibial nerves (PTN) somatosensory evoked potentials (SSEPs).

Methods: The SSEPs were performed by each PTN or the MN using square wave pulses at 15 mAintensity.

Results: Well-resolved MN-SSEPs were seen with stimulation of either arm. The principal peaks of N20, and P22 were 17, and $21 \mathrm{msec}$ for both upper extremities. The principal peaks of P37 and N45 were 35, and $46 \mathrm{msec}$ for both lower extremities. No side-to-side latency difference was noted. The MRI scan finding was a nondisplaced L1 fracture without spinal canal compromise

Conclusions: In spite of paraplegia, contradictory clinical findings, normal neurophysiologic tests, and normal neuroradiologic findings are positive criteria for paraplegia/quadriplegia with psychogenic etiology.

\section{P-075}

\section{Electromyography as a screening tool for myotonic muscular dystrophy}

\section{F.H. Jacques (Hull, Quebec)}

Background: Once an index case has been confirmed via DNA testing as having myotonic muscular dystrophy, screening family members at risk becomes the next priority. Will clinical and electromyography (EMG) evaluations suffice or should all individuals at risk undergo genetic testing?

Methods: 21 individuals at risk for having myotonic muscular dystrophy were screened prospectively using clinical examination and EMG. All patients with "normal" results underwent genetic testing.

Results: Seven had abnormal clinical and EMG examinations. Only one had an abnormal EMG with a normal clinical exam. Seven of 13 individuals tested positive despite a normal EMG. All were of the E0 category (50 to 200 trinucleotide repeats).

Conclusions: EMG is relatively insensitive in diagnosing clinically asymptomatic patients with myotonic muscular dystrophy. All individuals at risk with normal EMG should undergo genetic testing.

\section{P-076}

Usefulness of simultaneous intraoperative monitoring of anal and bladder sphincteric EMG during neurosurgery

A.V. Krassioukov, R. Sarjeant, S. Louissi, H. Arkia, M.G. Fehlings (Toronto, Ontario)

Background: Electrophysiological monitoring of external anal (EAS) and bladder (EBS) sphincteric EMG can potentially reduce the risk of perioperative neurological deficits following neurosurgery. However, the rationale for monitoring both EAS and EBS remain controversial.

Methods: We analyzed our experience with intraoperative monitoring of evoked and spontaneous EAS and EBS EMG in 64 consecutive patients (age 15-90 y, 35 M, 29 F). Urethral ring and anal sphincter electrodes (Medtronic Dantec) were employed. EMG from quadriceps femoris, tibialis anterior and gastrocnemius muscles were also recorded. In most cases EMG was recorded bilaterally, thus ensuring that all segments from L2 to S2 were monitored continuously.

Results: Spontaneous EMG during the surgery was observed in 19 cases $(31 \%)$. In four cases, spontaneous EMG activity was registered from EAS and EBS simultaneously. Spontaneous EMG was associated with surgical manipulation at the site of surgery (tumor resection, manipulation of filum 
terminale etc). Moreover, in one case, evoked EMG was recorded only from EAS but not from EBS during stimulation of filum terminale.

Conclusions: In previous investigations authors proposed that the recordings of EMG of the EAS might be able to substitute for those of the EBS, as the pudendal nerve from S2-S4 segments innervates both sphincters. Our findings suggest that simultaneous recording of the EMG from both muscles is essential during the neurosurgical manipulation at the lumbosacral region in order to prevent perioperative bowel and bladder dysfunctions.

\section{P-077}

\section{Motor responses to dorsal rootlet stimulation during selective posterior rhizotomy are consistent}

\section{S. Mittal, J-P. Farmer, C. Poulin, K. Silver (Montreal, Quebec)}

Background: Recently, controversy regarding the reliability of intraoperative stimulation of dorsal rootlets during SPR has emerged. The goal of this study was to determine whether motor responses to electrical stimulation were reproducible.

Methods: Dorsal roots (L2-S2) were stimulated with a 1second $50-\mathrm{Hz}$ train in 77 patients. A second stimulation run of the entire dorsal root $(\mathrm{n}=752)$ was carried out prior to division into rootlets. Rootlets were individually stimulated and sectioned according to extent of 'abnormal' electrophysiological propagation. Motor responses were recorded by both electromyography and a physiotherapist and assigned a grade of 0 to 4+. Grade difference between the first and second stimulation trains was determined.

Results: Statistical analysis demonstrated a clear consistency between stimulation runs both in the EMG and physiotherapy motor responses. Over $93 \%$ of dorsal roots had either no change or one grade difference between the two trials. The vast majority of dorsal roots assigned a grade 4+ at the first trial maintained the same maximally 'abnormal' electrophysiological response during the second stimulation run.

Conclusions: This study suggests that currently used techniques are reproducible and reliable for 'abnormal' rootlet selection. Intraoperative electrophysiological stimulation can be valuable in achieving a balance between elimination of spasticity and preservation of underlying strength.

\section{P-078}

\section{Short-latency somatosensory evoked potentials to median nerve stimulation in patient with intramedullary lesion: origin of $\mathrm{N}-13$ component}

\section{R. Sarjeant, S. Louissi, C. Tator, A. Krassioukov (Toronto, Ontario)}

Background: Intraoperative monitoring has become a standard of care during neurosurgical procedures. In particular, somatosensory evoked potentials (SSEP) are commonly used to monitor spinal cord function. The recordings of SSEPin patients with CNS lesions challenge the technologist and neurosurgeon in interpretation of the recorded parameters. Previously, numerous reports addressed issues of the origin of the N-13 component of the short latency SSEP.

Methods: Recording electrodes for the SSEP were positioned at the following sites: scalp electrodes: $\mathrm{CPz}$ (1), FPz (2), CP3 and CP4 $(3,4)$; posterior neck electrode Cv2 (5), and Erb's electrodes bilaterally $(6,7)$.

Results: We present a case of a 65-year-old woman who underwent a posterior fossa craniotomy for cervicomedullary junction granuloma. MR findings showed an intraxial mass lesion on the right side in the upper cervical cord at the cervicomedullary junction. Intraoperatively, during the collection of baseline recordings of the SSEPelicited by median nerve stimulation, the patient showed significant asymmetry of the short latency component $\mathrm{N}-13$, elicited by stimulation of the right median nerve. At the same time, the Erb's point potentials were symmetrical on both sides.

Conclusions: These electrophysiological findings provide further support that the $\mathrm{N}-13$ component originates at the level of cervicomedullary junction. This case is particularly interesting for two reasons. First, the focal lesion was present at the level of the right cuneate nucleus. Second, the presence of strong electrophysiological findings correlated with the lesion. These revealed the facts that the most conspicuous anatomical structure for the N13 generator is the cuneate nucleus.

\section{P-079}

\section{Value of early nerve conduction studies with focal weakness}

P.J. Sweeney, A.J. Wilbourn (Cleveland, Ohio, USA)

Background: Generally, electrodiagnostic (EDX) studies are performed three or more weeks after onset of symptoms so that axon loss lesions can be detected and localized by needle examination. Occasionally, however, motor nerve conduction studies (MNCS) performed very early in the course prove helpful.

Methods: Retrospective review of clinical/EDX findings in three patients; in each, MNCS performed 2-3 days after sudden onset of focal weakness were crucial for localization/diagnosis.

Results (cases): 1) Young woman with weakness in right radial/ulnar distributions; radial and ulnar MNCS showed no conduction blocks (CBs). A brain magnetic resonance imaging (MRI) subsequently demonstrated contralateral hemisphere vascular lesion. 2) Elderly man with painless right foot drop following lumbar laminectomy; peroneal MNCS, (recording tibialis anterior), revealed no CB at fibular head (the surgeon's diagnosis). A severe L5 radiculopathy subsequently demonstrated. 3) Elderly man with right hand weakness scheduled for $\mathrm{C} 8$ root decompression following MRI showing multilevel canal stenosis, ulnar MNCS showed a prominent CB at elbow. Surgery canceled.

Conclusions: In selected cases MNCS done early can be helpful for localization, by revealing either a $\mathrm{CB}$, or the absence thereof. Noteworthy is that, at this early stage, if CB is found, its underlying pathology (demyelination vs axon degeneration) cannot be determined. 


\section{P-080}

Finger tapping in a young male producing centroparietal spike-like discharges seen on electroencephalography

\section{Toth, N. Lowry (Saskatoon, Saskatchewan)}

Objective: To describe the phenomenon of finger-tapping related electroencephalography (EEG) changes seen in a young male patient.

Background: Spike-like discharges on EEG have rarely been reported in the European literature in association with finger tapping.

Methods: A seven-year-old male patient was referred for a 10 month history of clinical episodes consistent with absence epilepsy and one generalized seizure at age six years. Examination was unremarkable. EEG was ordered as a clinical investigation.

Results: EEG demonstrated the presence of contralateral centroparietal spikes seen in either hemisphere in association with unilateral finger tapping only. Discharges are reminiscent of somatosensory evoked potentials. Significance of this EEG phenomenon is unclear.

Conclusions: Finger tapping is associated with a contralateral hemispheric EEG phenomenon in this male patient. The incidence of this EEG finding is unknown. Functional magnetic resonance imaging of the cortex during finger tapping is planned.

\section{NEURO-ONCOLOGY}

\section{P-081}

\section{Juxtaposition of an ectopic corticotroph cell adenoma of the spheniod sinus with orthotopic intrasellar corticotroph cell hyperplasia in a patient with Cushing's disease}

\section{Al-Gahtany, J. Bilbao, K. Kovacs, E. Horvath, H. Smyth} (Toronto, Ontario)

Background: Ectopic pituitary adenomas (EPA) are rare and their association with orthotopic corticotroph hyperplasia has not been reported.

EPA refers to a condition in which an adenomatous pituitary tissue is present outside the sella turcica and is not in continuity with the sellar pituitary gland. Careful radiologic, intraoperative and postmortem examination of the relationships between the adenoma, the diaphragm sellae, the pituitary stalk and the pituitary gland is required to make this diagnosis.

Method: The case of 30-year-old woman with clinical and biochemical evidence of Cushing's disease is reported.

Results: The pre-operative MRI revealed asymmetrical inhomogeneity of the pituitary gland suggestive of localized adenoma. It also showed what was thought to be a small sphenoid polyp. The later lesion was found postoperatively to be an ectopic corticotroph adenoma. The pituitary gland, which was free from any tumor, showed diffuse unilateral corticotroph hyperplasia.

Clinical, radiological, laboratory and histopathological data are presented. Review of the topic and discussion of the etiology of the pituitary hyperplasia are provided.

Conclusion: Ectopic pituitary adenomas are relatively rare. They are found in places where embryonic remnants of Rathke's pouch or misplaced cells from pars tuberalis are located, the commonest of which being the sphenoidal bone. Pituitary corticotroph hyperplasia associated with EPA has not been described previously. While the exact mechanism of this association is not known, possible mechanisms are discussed. EPA should be kept in mind in all cases of Cushing's disease with normal sellar imaging and in whom previous surgical exploration yielded a negative result.

\section{P-082}

\section{Brown tumors of the skull base}

M. Al-Gahtany, M. Cusimano, W. Singer, J. Bilbao, K. Kovacs (Toronto, Ontario), Thomas Marotta (Vancouver, British Columbia)

Background: The natural history of brown tumor of the skull base, a rare and benign condition, has not been adequately documented, particularly after nonsurgical treatment and over a long follow-up period.

Methods: 38-year-old healthy female presented with headache and diplopia. Her examination is unremarkable apart from papilloedema and bilateral 6th nerve palsy. Preoperative imaging revealed cystic lesion in the ethmoidal sinus and the laboratory results where consistent with hyperparathyroidism which was confirmed by radioisotope scan that led to surgical resection of parathyroid adenoma as well as biopsy of the skull base lesion. Response to treatment was sustained over many years.

Results: This case presents the first reported case of brown tumor of the ethmoidal-frontal skull base in primary hyperparathyroidism. The correlation among clinical, radiological, and pathological findings in our patient are discussed. We review the literature on brown tumor of the skull base and discuss its diagnosis and treatment.

Conclusion: Brown tumor of the skull base, although rare, should be considered in the differential diagnosis of skull base lesions. Surgical resection is often unnecessary but a biopsy should be performed to exclude other types of lesions. Once the diagnosis is established, brown tumors usually respond to treatment of the underlying condition. Our case shows the rapid, sustained response to the cure of hyperparathyroidism.

\section{P-083}

Outpatient craniotomy for brain tumour resection: a prospective feasibility study with 46 patients

\section{Bernstein (Toronto, Ontario)}

Background: The scope of minimally-invasive outpatient surgery is broadening. In late 1996 the author initiated a pilot study of outpatient craniotomy for brain tumour resection (OCBTR).

Methods: Patients requiring craniotomy for resection of an intra-axial tumour were offered voluntary entry into the study. The protocol consists of the patient's arrival at the Day Surgery 
Unit (DSU) at 6:30 a.m. for MRI for an image-guidance system. After surgery the patient spends four hours in the recovery room and is discharged by the surgeon from the DSU at 6:00 p.m. with home-care nursing visits planned for 11:00 p.m. that evening and the next morning.

Results: There were 46 patients in the intent-to-treat group. Pathology was 19 glioma, 21 metastasis, six other. Five patients were converted to in-patients so the successful protocol completion rate was $41 / 45=89 \%$. There were five complications in the intent-to-treat group $(11 \%)$. No patient voiced dissatisfaction with the procedure and no patient's health was compromised by entry into this study.

Conclusions: OCBTR is safe for selected patients. There are also a number of real and theoretical reasons why this procedure could even be superior to standard inpatient craniotomy. Prospective studies with satisfaction instruments are planned to ascertain that there are no hidden human costs of this procedure.

\section{P-084}

\section{Pediatric myxopapillary ependymoma}

C. Chaalala, A. Turmel, P. Gould (Quebec, Quebec)

Myxopapillary ependymomas are rare in the pediatric population. The clinical diagnosis of these lesions is very often delayed and difficult. Radiological images are rather typical but in this age group, these lesions tend to be very large. We present a group of six patients with myxopapillary ependymoma. Five had gross total resection and one presented with dissemination and had partial resection.

Clinical presentation, radiological diagnosis and histological characteristics will be discussed. Adjuvent therapy and prognosis will be entertained.

\section{P-085}

\section{Unusual CNS metastases}

C. Chaalala, J. Bazinet, A. Turmel, G. Milot, C. Picard, P. Gould (Quebec, Quebec)

Cerebral metastasis are the most common brain tumor seen clinically in neurosurgery, and of patients harboring a systemic cancer, approximately $25 \%$ have intracranial metastasis.

The two most common primary sites are lung and breast. Oesophagus and thyroid are the origin of cerebral metastasis in less than $6 \%$ of cases, and only sporadic cases of thymoma metastasizing to CNS have been reported.

We present four cases of unusual CNS metastasis, two originating from a thymoma, one from a thyroid carcinoma, and one from an oesophageal carcinoma.

We will discuss clinical presentation, imaging, histology and management of these cases.

\section{P-086}

\section{Pediatric spinal epidural tumors: an institutional review}

Jean-Francois Chevalier, Jean-Pierre Farmer, José L. Montes, Augustin M. O'Gorman, Carolyn Freeman (Montreal, Quebec)

Background: Spinal epidural tumors account for $3 \%$ to $5 \%$ of solid tumors of childhood. These tumors in this specific population have different presentation and prognosis than in the adult population, and few studies have addressed this issue.

Method: Retrospective chart review of surgical patients selected from the Montreal Children's Hospital Tumor Registry.

Results: 11 cases were available for review, comprising seven neuroblastomas and four tumors of other origin. At the time of primary assessment, the median follow-up was seven years. Two mortalities were recorded after 4.5 and 94 months. The neurological recovery was excellent among survivors, with six patients ambulating independently, and three with assistance, despite severe initial presentation. The advent of MRI did not accelerate the diagnosis. Long-term scoliosis remains a major complication in these patients, with four children requiring surgical correction.

Conclusions: Patients with these tumors have an excellent survival, with good potential for neurological recovery despite a severe impairment at presentation. In this series, MRI failed to obtain a quicker diagnosis and select for spinal decompression a population with least neurological impairment. Postoperative scoliosis remains a major long-term complication and is likely explainable by the young age of these patients.

\section{P-087}

\section{A comparison of quality-of-life in support-group and nonsupport-group patients with pituitary adenoma}

\section{P. Kan, M.D. Cusimano (Toronto, Ontario)}

Background: Pituitary adenomas and/or their treatment may have a significant influence on patients' quality of life (QOL). Our previous work involved the development of a QOL instrument specifically for patients with pituitary adenoma. Patients who attended patient-support groups were primarily involved in previous studies. The purpose of this work was to compare the QOL of patients with pituitary tumours attending support groups (SG) with a group of unselected patients visiting neurosurgeons'offices (OP).

Methods: QOL questionnaires were administered to 47 support-group and 22 nonsupport-group patients. Patients were asked to rank the importance to their own QOL for each item in the instrument on a 7 point scale ( $1=$ excellent, $7=$ very poor). Subscale and total scores on the Pituitary QOL (PQOL) instrument were compared by t-tests.

Results: The PQOL instrument was well accepted by patients although OP patients took longer to complete the instrument (15 vs. 11 minutes). SG and OP patients had similar mean PQOL scores: general health $(3.57,4.18 ; \mathrm{p}=0.41)$; social and family well-being $(3.72,3.63 ; \mathrm{p}=0.45)$; emotional well-being $(1.18$, $1.24 ; \mathrm{p}=0.68)$; health problems related to pituitary tumour $(3.37$, $3.58 ; \mathrm{p}=0.78)$; relation with health professional $(3.79,3.36$; 
$\mathrm{p}=0.34)$; total score $(21.4,25.4 ; \mathrm{p}=0.3)$. Test re-test reliability was excellent $(\mathrm{OP}=0.86 ; \mathrm{SG}=0.88)$. Scores one month apart were not significantly different in either group (OP $\mathrm{p}=0.69 ; \mathrm{SG}$ $\mathrm{p}=0.73$ ).

Conclusion: SG and OP patients showed QOL equivalent scores. Further evidence for the validity and reliability of the developed PQOL instrument was demonstrated.

\section{P-088}

\section{A unique lesion of the skull: primary natural killer cell lymphoma}

\section{N. Al-Shafai, M. Cusimano, S. Deodhare (Toronto, Ontario)}

Background: Neurosurgeons must consider many diagnostic and therapeutic issues when faced with patients with primary lesions of the skull. The purpose of this paper is twofold: 1) to present the first reported case of natural killer cell lymphoma of the skull; 2) to review the literature of skull lesions.

Methods: Case report and literature review.

Results: An 81-year-old hypertensive woman presented with a three month history of an enlarging left parietal mass lesion and hypercalcemia of unknown etiology. Examination showed a soft, nontender, fixed, erythematous lesion of the left parietal region without lymphadenopathy and neurological deficits. CT scan demonstrated a well-defined lytic lesion centered in the skull with both epidural and subcutaneous extension. Open biopsy showed a cellular tumor with monomorphous large to medium sized atypical cells with inconspicuous nucleoli. They were only positive for CD45 and CD56 and negative for T and B cell markers. Molecular analysis showed no evidence of $\mathrm{T}$ cell receptor rearrangement.

Conclusions: CD56-positive lymphoma consists of four subtypes, which tend to occur in unusual extranodal sites such as the nasopharynx, CNS, muscle and skin. Our case is the first reported to occur in the skull. How the lesion fits into the differential diagnosis of skull lesions will be presented along with a literature review and management algorithm for skull lesions.

\section{P-089}

\section{Development and validation: quality of life questionnaire for Cushing's disease patients}

\section{A. Marchie, M.D. Cusimano, H.S. Smyth, Fateme Salehi (Toronto, Ontario)}

Introduction: Patients with pathologically-verified Cushing's disease (CD) have a range of symptoms such as obesity and depression. Understanding the impact of CD on quality of life (QOL) is important in choosing appropriate treatment. No such QOL assessment exists for these patients. The purpose of this study is to develop and validate a CD-specific health-related quality of life (HRQOL) questionnaire (QU).

Method: Focus group interviews and literature review were used to develop a QU with 177 items. These items were sorted into five domains: physical, cognitive, social, emotional, and medical. 22 patients completed the QU and feedback was given. Impact scores were based on a 7-point scale: 1=least; 7=most affected. T-tests $(\mathrm{p}<0.05)$ were used to compare the means of each domain. Validity was assessed by Pearson correlation coefficient between the QU and the Karnofsky Scale (KPS).

Results: Mean scores and standard deviations for each domain were: physical $(4.29,1.46)$; cognitive (3.91, 0.57); social (2.97, 1.23); emotional (5.07, 1.11); and medical (3.50, 1.72). Validity coefficient was 0.63 for general health (KPS). Physical $(\mathrm{p}=0.028)$ and emotional health $(\mathrm{p}=0.0002)$ had greater impact on QOL than other domains. Despite having less impact, cognitive health $(p=0.035)$ and medical treatment $(p=0.029)$ were rated as highly relevant to QOL; the opposite was found for physical health $(\mathrm{p}=0.02)$. The social domain was least affected by $\mathrm{CD}$ and had the least relevance to QOL. $73 \%$ of the patients felt the QU captured an accurate sense of their QOL; $14 \%$ thought otherwise, and $13 \%$ did not give an opinion.

Conclusion: A HRQOL QU was developed for patients with Cushing's disease. Preliminary evidence for validity was demonstrated. Domains may unequally influence a CD patient's perception of QOL. Dual scaling will be explored as a means to reduce the number of QU items and to help understand better the meaning of the responses.

\section{P-090}

\section{Primary diffuse leptomeningeal gliomatosis}

A. Varma, M.D. Cusimano, J.M. Bilbao, L.N. de Tilly (Toronto, Ontario)

Background: Primary diffuse leptomeningeal gliomatosis (PDLG) is a very rare disorder. It is one of the causes of leptomeningeal enhancement. So far 23 cases have been described in the English literature.

Method: This report describes a 46-year-old female with PDLG. After a detailed clinical and radiological evaluation, diagnosis was established by open biopsy.

Results: This 46-year-old female presented with a four year history of seizures, headache and failing vision. Fundus examination showed bilateral pale discs, likely a result of direct optic nerve compression. Neuroimaging revealed hydrocephalus and diffuse meningeal enhancement. Cerebrospinal fluid (CSF) examination was unremarkable. An antemortem histological diagnosis was established, by open biopsy. She recovered well from surgery, but deteriorated while undergoing further treatment. Subsequent ventriculoperitoneal (VP) shunt resulted in inexplicable deterioration and death. Post shunt computerized tomogram (CT) scan revealed diffuse hypoxic injury.

Conclusion: A literature review reveals that a nonspecific prodromal phase is usually followed by a progressive downhill course. Neuroimaging may be negative initially. CT scan and magnetic resonance imaging (MRI) show focal or more often diffuse meningeal enhancement. CSF may show pleocytosis and raised proteins. Early diagnosis followed by aggressive treatment may result in remission. This case is unique, as such a long history prior to presentation and optic nerve atrophy have not been described before. Deterioration following VP shunt is difficult to explain. Changes in pressure equation(s), amongst different compartments, following the shunt could have critically compromised the blood supply. 
P-091

Indications for adjuvant therapy in the management of nonfunctional pituitary adenomas: a 20 year experience

S. Hentschel, D.E. Griesdale, G. Wilkins (Vancouver, British Columbia)

Background: Current microsurgical techniques and evolving adjuvant therapies require that surgical results and indications for adjuvant therapy be examined as these tumours have received relatively little attention in the recent neurosurgical literature.

Methods: The records of all patients undergoing a surgical procedure for a sellar lesion by a specialty pituitary surgeon (DEG) from January 1979 to December 1999 were reviewed retrospectively.

Results: There were 432 procedures of which 158 (37\%) were for a clinically nonfunctional adenoma. The patients were divided into three groups: I) those that received no postoperative radiotherapy (77 patients); II) those that received prophylactic postoperative radiotherapy (66 patients); and III) those that received radiotherapy only after the tumour had recurred (15 patients). The control rates were $98.7 \%, 98.5 \%$, and $86.7 \%$ for groups I, II, III, with an average follow-up of 1.7, 5.3, and 5.6 years, respectively. In the subgroup of patients with confirmed postoperative complete resection on imaging (47 patients), there were two recurrences. In the group of 90 patients with confirmed partial resections, 18 out of the 20 recurrences occurred in patients who had not received postoperative radiotherapy (49 patients).

Conclusions: Radiotherapy may be withheld in those patients whose tumours are completely resected but should be given prophylactically for partial resections based on the age of the patient and general medical condition. The control rate deteriorates for patients who receive radiotherapy only after the tumour recurs.

\section{P-092}

\section{Molecular immunohistochemical diagnosis of an anaplastic focus within a pilocytic astrocytoma}

\section{T.G. Mainprize, J.M. Bilbao, L. Roncari, A. Guha (Toronto, Ontario)}

Background: Astrocytomas are heterogenous tumors, both histologically and molecularly. Molecular markers of anaplasticity were examined immunohistochemically in a specimen where there was progression from a diffuse pilocytic astrocytoma.

Methods: Immunohistochemistry was performed on a single tumour specimen.

Results: The molecular characteristics of anaplasticity were clearly noted including: 1) Higher MIB-1 immunopositivity (10\% vs $0 \%)$; 2) Increased vascularity, with increased number of FactorVIII positive vessels and increased expression of Vascular Endothelial Growth Factor (VEGF) by the tumor cells; 3) Overexpression and activation of Epidermal Growth Factor Receptors (EGFR); 4) Expression of the mutant EGFRVIII by the GBM focus. The two signal transduction pathways implicated in the growth of human GBMs and utilized by aberrant EGFRs, mediated by PI3-Kinase and p21-ras, were also examined. Activation of the PI3-Kinase and p21-ras pathways were inferred by increased activation of their main downstream effectors Akt/PKB and MAPK, respectively. Furthermore, there was loss of PTEN/MMAC1 expression. Expression of p53 and neurofibromin were not altered.

Conclusion: This is a rare molecular immunohistochemical study undertaken on a single specimen harboring both an anaplastic focus and a pilocytic astrocytoma. It demonstrates our ability to identify molecular changes in tumors using standard immunohistochemical techniques and may play a role in guiding diagnosis and treatment.

\section{P-093}

Pineal apoplexy leading to an expanding pineal cyst: case report

P.D. McNeely, V. Mehta, W.J. Howes, L. Resch (Halifax, Nova Scotia)

Background: Pineal apoplexy has been reported on occasion in the literature. This is a rare syndrome which results from hemorrhage into a pineal lesion.

Methods: Report of a case and review of the literature.

Results: A 12-year-old girl presented with a six-day history of headaches, nausea, vomiting, and syncopal episodes. Computed tomography (CT) performed at her referring hospital revealed a 10 by $14 \mathrm{~mm}$ hyperdense pineal region mass with hydrocephalus. At the time of arrival to our emergency department she was unresponsive. She was intubated and an external ventricular drain was placed. She awoke shortly thereafter and her hydrocephalus was treated definitively with an endoscopic third ventriculostomy. Serum alphafetoprotein and human chorionic gonadotropin levels were within normal limits. Magnetic resonance imaging (MRI) was then performed and suggested there had been a recent hemorrhage into a pineal region lesion. She recovered completely and was discharged home. A repeat MRI scan seven weeks later revealed a large cystic lesion with resolution of the blood products. Surgical resection of this lesion was therefore recommended to rule out a neoplasm. The final histological diagnosis was that of a pineal cyst with evidence of recent hemorrhage.

Conclusions: Symptomatic pineal cysts presenting with hemorrhage are quite uncommon. Hemorrhage within the pineal gland may be involved in the pathogenesis of enlarging pineal cysts.

\section{P-094}

\section{Diagnosing pediatric brain tumors. How did we do between 1995-2000 in the Maritimes?}

V. Mehta, A. Chapman, P. Yhap, W.J. Howes (Halifax, Nova Scotia), S. Walling (St. John, New Brunswick)

Background: Central nervous system tumors are one of the most common causes of pediatric cancer. Historically, the mean time from symptom onset to diagnosis has been in the four to six month range. This time period is substantially higher than other forms of cancer such as leukemia. 
Methods: A combined retrospective/prospective study was undertaken of children, aged 0-16 years with primary brain tumors between 1995-2000 within the Maritimes. General patient demographic information as well as tumor specific data were collected. Structured parent interviews and chart reviews were utilized to acquire information on duration and type of symptoms prior to diagnosis, the number of consultations prior to diagnosis as well as other covariates.

Results: Between 1995-2000, we treated 105 cases of primary pediatric brain tumors. Fifty-four percent (57/105) cases involved the male sex. Tumors were slightly more common in the infratentorial compartment, with $53 \%(56 / 105)$ of the cases involving posterior fossa structures. Data regarding time to diagnosis, number of consultations prior to diagnosis as well as other variables will be presented.

Conclusions: We believe that an understanding of factors that lead to the diagnosis of children with brain tumors is worthy information. The results of this study will hopefully shed light on the process by which these children enter the medical system within the Maritimes.

\section{P-095}

\section{Clinical trials in the photodynamic therapy of malignant brain tumors}

Paul Muller, Brian Wilson, Lothar Lilge, Victor Yang (Toronto, Ontario), Tim Fullagar, Fred Hetzel, Q. Chen (Denver, USA), Robert Fenstermaker (Buffalo, USA), Robert Selker (Pittsburgh, USA), Judith Abrams (Detroit, USA).

Background: Photodynamic therapy consists of the administration of a photosensitizer followed by the photoillumination of the sensitized tissue with light of the appropriate wavelength. We have treated more than 200 patients with malignant brain tumors with PDT.

Method: In a phase 2 study we have treated 101 patients with malignant brain tumors, 86 of whom had a glioma. Photofrin 2$\mathrm{mg} / \mathrm{kg}$ was administered i.v. 12-36 hours prior to craniotomy. All patients underwent a resection of tumor or tumor cyst drainage. The resultant tumor cavity was illuminated using a KTPpumped dye laser. The light dose ranged from 8 to $150 \mathrm{~J} / \mathrm{cm}^{2}$. The surgical mortality rate was $3 \%$.

Results: Regression analysis revealed that pathology $[\mathrm{p}=0.007]$, Karnofsky score [ $\mathrm{p}=0.024]$, and light dose $[\mathrm{p}=0.016]$ were significant predictors of survival outcome.

Conclusions: We concluded that PDT was safe in patients with newly diagnosed or recurrent supratentorial malignant gliomas. Higher light doses were superior to lower light doses and a significant prolongation of survival occurred in selected patients when an adequate light dose was used.

In March 1998, we initiated two randomized prospective trials. The first was to determine if the addition of PDT to standard therapy [surgery, radiation and chemotherapy] prolongs the survival of patients with newly diagnosed malignant astrocytic tumors. The second was to determine whether high light dose PDT[120 J/cm ${ }^{2}$ ] is superior to low light dose PDT [40 $\mathrm{J} / \mathrm{cm}^{2}$ ] in patients with recurrent malignant astrocytic tumors. As of December 2000, 115 patients have been recruited to these studies. The pathologic distribution is as follows: GBM: 73, anaplastic oligo-astrocytoma: 23, malignant astocytoma: 5, other: 14. With the exception of laser failure and skin toxicity, postoperative complications were evenly distributed. The perioperative mortality was $1.7 \%$.

\section{P-096}

\section{Subependymomas: report of five symptomatic cases Paul Muller, Juan Bilbao (Toronto, Ontario)}

Background: Russell and Rubenstien noted that subependymomas occur in an intraventricular situation, are frequently multiple, have sharp demarcation and a lobulated appearance, are slow growing and usually display noninvasive behaviour.

Method: We have operated on five patients with symptomatic subependymomas among a group of 20 adult patients with intracranial ependymomas in our series of 1200 intracranial gliomas.

Results: There were four males and one female. Their ages were $24,35,69,71$ and 71 respectively. The younger two had lateral ventricular lesions and the older three had 4th ventricle and vermal lesions. Subtotal but radical resection was carried out in all five. In three, the lesion resulted in symptomatic recurrence. One died [three years postoperatively from recurrent tumor] and two came to re-operation which showed a pathologic progression to a more aggressive ependymoma in each of the two patients in three and five years, respectively.

Conclusion: Although many patients with subependymomas are indeed asymptomatic, those that present with neurological findings requiring surgical treatment do require careful followup because of the risk of recurrence and progression.

\section{P-097}

Acoustic neuroma surgery: psychological, social and economic impacts on patients and their caregivers

\section{N. Panu, D. Rowed (Toronto, Ontario)}

Background: The objectives of this study were to investigate the impact of acoustic neuroma (AN) surgery on activities of daily living (ADL), social, psychological, occupational facets of the life of the patient as well as the patient's primary caregiver, an impact that has received little previous attention. A further attempt was made to discover predictors of postoperative impact from preoperative variables such as age, sex, tumour size, and surgical approach.

Methods: A questionnaire was sent to all AN patients treated at Sunnybrook and Women's College Health Sciences Centre. One hundred fifty patients and 112 caregivers responded.

Results: Patients were negatively affected to various degrees in ADL, social, psychological, occupational aspects of their lives. These effects, however, could not be reliably predicted from preoperative variables. The patient's primary caregivers were also negatively affected, which again could not be predicted from preoperative variables, although caregivers for both positively and negatively affected patients were impacted more than those of patients not affected by surgery.

Conclusions: AN surgery has a considerable affect on 
patient's quality of life. Moreover, caregiver impact is significant, an aspect of management not previously studied. Neither of these can be predicted from preoperative variables. Patients and their caregivers should be prepared and informed preoperatively regarding the consequences of surgery on their quality of life.

\section{P-098}

\section{CDNA microarray analysis of pediatric medulloblastomas}

Paul C. Park, Michael D. Taylor, Jeremy Squire, Todd Mainprize, Laurence Becker, James T. Rutka (Toronto, Ontario)

Background: While medulloblastoma is the most common primary pediatric brain tumour, little is known regarding its molecular pathogenesis. The recent availability of cDNA microarrays permits the evaluation of the expression levels of hundreds or even thousands of genes compared to a reference tissue within the confines of a single experiment.

Methods: We examined the expression profile of six primary medulloblastomas as compared to normal cerebellum using a nylon-based array with over 1200 genes thought to be important in neoplasia (Atlas Human Cancer Array, Clonetech).

Results: We found consistent over/under expression of a number of interesting genes in medulloblastoma. These genes were consistently over/under expressed in all or most of the tumour samples suggesting that their expression level is relevant to the phenotype of medulloblastoma. Interesting genes found to be over/under expressed include genes involved in regulation of the cell cycle (Cyclin D2, CDK4,CHES-1), invasion and migration (Ezrin, Metallothionein 3, Tenascin), putative tumour suppressor genes (CDI46 Mel-CAM, BIN-1), and regulators of apoptosis (Death Receptor 3 ).

Conclusions: We have shown that cDNA microarrays can identify pathways and genes altered in cerebellar medulloblastoma. Genes identified by this screening technique suggest many further avenues of research to better understand the biology of this tumour.

\section{P-099}

\section{Medulloblastoma in a patient with Rubinstein-Taybi Syndrome}

\section{Michael D. Taylor, Todd G. Mainprize, Larry Becker, James} T. Rutka, James Drake (Toronto, Ontario)

Background: Patients with Rubinstein-Taybi Syndrome (RTS) have multiple congenital anomalies including decreased cognition, growth retardation, broad thumbs and toes, abnormal facies and microcephaly. It has been suggested that RTS patients also have an increased incidence of malignancy especially in the CNS. RTS is due to germline mutations in the CREB-binding protein $(C B P)$ gene on chromosome 16 that functions in many signal transduction pathways including Sonic Hedgehog signaling, Wnt signaling and p53 mediated transcription.

Methods: We reviewed the records of the described case.

Results: We present a three-year-old male who is the product of a full term delivery after an unremarkable pregnancy. The proband presented with broad thumbs and toes as well as a ventricular septal defect and global developmental delay including mild gait and balance problems. An MRI for assessment of developmental delay revealed a mass in the fourth ventricle. Surgical resection of the mass revealed a typical medulloblastoma with a high mitotic rate and synaptophysin immunopositivity.

Conclusions: This case supports the association between RTS and malignancy and specifically with medulloblastoma. The role for screening MRI to diagnose CNS neoplasia in patients with RTS remains undefined.

\section{P-100}

\section{Unusual pituitary tumor: salivary gland-like tumor of} the sella

W.R. van Furth, A.Varma, H.S. Smyth, J. Goguen, E. Horvath, K. Kovacs, M.D. Cusimano (Toronto, Ontario)

Background: Salivary gland rests are known to occur in the pituitary gland. We present a patient with a sellar tumor arising from these salivary gland remnants.

Case report: This 60-year-old man presented with progressive anorexia, fatigue, headache, polyuria and visual field defects. The MRI showed a $2.5 \times 2.5 \mathrm{~cm}$ sellar mass, with suprasellar and posterior fossa extensions. On repeated scans the tumor had grown $6 \mathrm{~mm}$ in three months. Based on endocrine and imaging studies the diagnosis of a nonfunctioning pituitary mass was made. The patient underwent transsphenoidal debulking of his adherent and invasively growing sellar tumor. The patient died eight days postoperatively of a ruptured thoracic aneurysm.

Pathology: The surgical specimen showed an epithelial tumor forming glandular structures, without nuclear pleomorphism and infrequent mitotic figures. Immunostainings for cytokeratin, alpha-1-antitrypsin and chymotrypsin were positive in the tumor cells. The electron microscopy showed granules accumulating within the apical portion of the cells. On the basis of the morphologic, immunohistochemical and ultrastructural features of the tumor the diagnosis of low grade acinic cell carcinoma was made. At autopsy no other tumors were identified.

Conclusion: The embryologic development of the pituitary gland provides an explanation for the occurrence of this tumor in the sella. In relation to the posterior pituitary, small seromucous glands of the salivary type can often be found communicating with Ratke's cleft remnants. The presence of salivary gland rests in the pituitary explains the occurrence of salivary gland-like tumors in the sellar region.

\section{P-101}

\section{Verotoxin treatment of malignant meningiomas}

W.R. van Furth, B. Salhia, M.F. de Rosa Jasson, M.D. Cusimano, C.A. Lingwood, J.T. Rutka (Toronto, Ontario)

Background: Although most meningiomas can be safely and effectively treated surgically, some meningiomas are notoriously hard to treat. Verotoxin 1 (VT) is an E. coli toxin, which was 
recently shown to have antineoplastic action by targeting the Gb3 receptor on tumor cells and tumor neovasculature. We therefore investigated VT as a potential treatment of malignant meningioma in our orthotopic meningioma xenograft model.

Methods, Results: We demonstrated that the Gb3 receptor is highly expressed by the malignant meningioma cell-line IOMMLee. In vitro, IOMM-Lee cells were highly sensitive to VT treatment $\left(\mathrm{LD}_{50}=0.4 \mathrm{ng} / \mathrm{ml}\right)$. Subcutaneous growing tumors of IOMM-Lee in nude mice were given an intra-tumor injection with VT, heat-inactivated VT (HI-VT) or phosphate buffered saline (PBS). VTtreated tumors showed a 50\% reduction in size, while HI-VT and PBS injected tumors continued to grow. However, after a week the VT treated tumors would start growing again, which could not be stopped by a second VT injection. The histology showed marked central necrosis in VT injected tumors. A single injection of VT in intracranial growing IOMM-Lee tumors doubled the survival of nude mice, compared to HI-VT or PBS. To explore the potential clinical relevance of this finding, 10 meningiomas were tested for $\mathrm{Gb3}$ expression. Four out of five malignant meningiomas showed Gb3 expression, with one tumor showing positive neovasculature.

Conclusion: Verotoxin treatment of malignant meningiomas is effective in our orthotopic xenograft model and warrants further exploration as a potential treatment.

\section{P-102}

\section{Intraoperative detection of glioblastoma multiforme by a multispectral fluorescence guidance system}

\section{Victor Yang, Paul Muller, Peter Herman, Brian Wilson (Toronto, Ontario)}

Background: Intraoperative identification of tumor can help to define the tumor extent and detect residual tumor tissue, which may increase the effectiveness of both conventional surgical resection and Photodynamic Therapy. We have developed a Multispectral Fluorescence Guidance (MFG) system that performs noncontact fluorescence spectroscopy and imaging quantitatively during brain tumor resection and photodynamic therapy (PDT). The MFG detected elevated red fluorescence from residual tumor tissue within the resection cavity, which was confirmed by histology and ex vivo spectrofluorometric measurements.

Method: The day before surgery, patients were administered Photofrin at $2 \mathrm{mg} / \mathrm{kg}$ i.v. Intraoperatively, tissue fluorescence was excited by $405 \mathrm{~nm}$ Mercury arc lamp and detected in the visible and near infrared wavelengths $(480 \sim 720 \mathrm{~nm})$. Both spectroscopic and multi-band imaging detection were made simultaneously. Fluorescence measurements were made during and after the surgical resection of tumor tissue, as well as before, during, and after Photodynamic Therapy. Biopsies were taken from the resection cavities, and examined histologically and photochemically to determine the pathology and concentration of Photofrin uptake.

Results: Normal tissues showed no porphyrin fluorescence, in contrast to tumor tissue which emits characteristic red fluorescence centered at $640 \mathrm{~nm}$. After resection, the MFG system was able to detect residual tumor with sub-millimeter resolution intraoperatively. During PDT, the MFG system detected photobleaching of Photofrin intraoperatively.

Conclusions: Surgical fluorescence microscopy is feasible intraoperatively. Photofrin used for PDT may serve as a fluorescence label for malignant gliomas. The MFG system can utilize such label for surgical guidance, which might enhance the completeness of tumor removal.

\section{P-103}

\section{Role of angiopoietins in angiogenesis of astrocytomas}

\section{G. Zadeh, L. Roncari, A. Guha (Toronto, Ontario)}

Background: Vascular Endothelial Growth Factor (VEGF) together with its endothelial specific receptors, is a major inducer of astrocytoma angiogenesis. Angiopoietins (Ang1/Ang2) are more recently identified angiogenic factors, with Tie 2 being their cognate receptor. We hypothesize that they are also involved in vascular growth of astrocytomas.

Methods: Expression profile of Angiopoietins and Tie2 was established in human astrocytoma specimens and established astrocytoma cell lines (EACL), using in-situ-hybridization, immunohistochemistry, western and northern blot techniques. Constructs were designed to over-express Ang1 and Ang2 in EACLs. Stable clones of these transfected cells were made into xenograft tumor models in 20 mice. Two months after tumor formation, the vascular pattern and growth of xenografts were studied.

Results: Ang1 was expressed in low levels in low-grade astrocytoma specimens and in higher levels in glioblastoma multiforme (GBM). Ang2 together with Tie2 receptor levels were abundant in proliferative tumor endothelium of GBMs. Two of three EACLs studied secrete Ang1 and as predicted no Ang2/Tie2. Xenografts with over-expressed Ang1 had increased growth and vascular density, while over-expressed Ang2 tumors demonstrated decreased vessel density and abnormal vessel structure.

Conclusion: Our data suggest that Angiopoietins modulate angiogenesis in astrocytomas, and hence targeting this pathway can yield an effective anti-angiogenic therapy for astrocytomas.

\section{SPINAL SURGERY}

\section{P-104}

\section{Microendoscopic discectomy - a review of 39 cases}

\section{E. Adeleke Badejo (Kearney, Nebraska, USA)}

Background: This is a review of 39 patients who underwent microendoscopic discectomy in our institution. The follow-up period ranged from six months to 32 months.

Method: All patients who had microendoscopic discectomy were sent questionnaires and their charts were reviewed. They were asked to grade their pain severity level pre-operatively and postoperatively on a scale of 1-10, their present functional status, and how long it was before they returned to work.

Results: There were 39 responses. In eight patients, the camera was replaced with the microscope. Thirty-one patients (79.5\%) had improvement in their pain status postoperatively. 
Eight patients had minimal improvement, no improvement, or were worse after surgery. $54 \%$ of the patients are pain-free and $24.3 \%$ have minimal pain. $8.1 \%$ were totally incapacitated.

Thirty-five patients were back to work; five at one week or less from the time of surgery. Of the four patients who are not back to work, three were on Workman's Compensation. There were seven patients on Workman's Compensation in this series.

Conclusion: Microendoscopic discectomy is a less invasive alternative to microdiscectomy in the management of patients with lumbar disc herniation.

\section{P-105}

\section{Benefit analysis of spinal cord stimulators: a retrospective study}

\section{T. Del Duca, A. Hochstein, L. Jacques (Montreal, Quebec)}

Objective: Spinal cord stimulation has become an effective treatment for failed back surgery syndrome (FBSS); increasing quality of life, reducing debilitating pain, and improving overall functional status of selected patients. The aim of the present study was to determine the cost-effectiveness of this treatment modality in Quebec.

Methods: A retrospective review of 67 patients was undertaken, 35 implanted and 32 nonimplanted. Costs for medical resources were tabulated. The two groups differed in follow-up time thus a cost adjustment was made to compensate for the difference.

Results: The implanted group had an average adjusted cost of $\$ 19,939$ versus an average cost of $\$ 27,659$ in the nonimplanted group at 64.5 months of follow-up.

Conclusion: Despite a substantial initial outlay of funds, spinal cord stimulators are a cost-effective method of treatment. In addition, we believe that on a long-term basis the implanted group will need even fewer resources than the final adjusted tally suggests.

\section{P-106}

Efficacy of an analgesic epidural paste following lumbar decompressive surgery: long-term follow-up of a prospective randomized double-blind controlled trial

Aaron S. Dumont (Charlottesville, USA), Nicholas Theodore, Volker K.H. Sonntag (Pheonix, USA), R. John Hurlbert (Calgary, Alberta)

Background: We have previously demonstrated the conclusive efficacy of a single-dose epidural analgesic "paste" in the control of postoperative pain in patients undergoing lumbar decompressive surgery followed for up to three months postoperatively. Subsequently, we have analyzed our long-term (one year) follow-up data to quantitatively evaluate any potential long-term effects of paste treatment.

Methods: Sixty patients undergoing routine elective lumbar decompressive surgery were randomized to receive either active or placebo paste. Patients were followed as inpatients and outpatients for 12 months postsurgery. Comparability between groups was ensured, and pain control was evaluated with multiple measures.
Results: Patients receiving active paste demonstrated significantly lower pain scores and better general health perception (as measured with the Short Form 36) for up to six weeks postoperatively. There were no significant differences between groups in these outcome measures at six months and 1year follow-up. Additionally, inpatient and outpatient oral narcotic consumption was lower in the active paste treatment group, compared to placebo paste group, for up to six weeks following surgery. No significant differences in narcotic consumption were ascertained at six months and one-year follow-up.

Conclusions: The application of an epidural analgesic paste during lumbar decompressive surgery significantly enhances pain control, reduces oral narcotic consumption and improves overall health perception for up to six weeks postoperatively. No significant differences were demonstrated between the treatment groups in these outcome measures at six months and one-year follow-up. Hence, the application of an analgesic epidural paste imparts significant benefit for up to six weeks following surgery without harmful long-term sequelae. Implementation of this efficacious and safe pain management strategy may evolve into a new standard of care in patients undergoing lumbar discectomy or laminectomy.

\section{P-107}

Quantitative CT analysis of atlanto-axial transarticular screw fixation: classification of surgical outcome with technical insights

\section{Benoît Goulet, Michael G. Fehlings (Toronto, Ontario)}

Background: CT scan with sagittal reconstruction best evaluates the adequacy of transarticular screw fixation. We present our result using a new classification for $\mathrm{C} 1-\mathrm{C} 2$ transarticular screw fixation. That classification is compared with an index of subluxation.

Methods: We reviewed 22 patients with postoperative C1-C2 CT with sagittal reconstruction to assess 42 screws after a transarticular screw fixation. The screw trajectory was classified in four categories by subdividing in thirds the facet joint. The index of subluxation is defined as the difference between the antero-posterior displacement of the odontoid and the atlantoaxial interval.

Results: We found a significant correlation between the adequacy of the screw position and the reduction of the subluxation. There was no case of vertebral, spinal cord or cranial nerve injury. The pars interarticularis height varied from $3 \mathrm{~mm}$ to $11 \mathrm{~mm}$ with a mean of $7 \mathrm{~mm}$ on the right side and 7.3 $\mathrm{mm}$ for the left side.

Conclusion: For proper position of C1-C2 transarticular screws we found that the reduction of the atlanto-axial subluxation is essential. The screw should be in the posterior third of the facet joint and pass in the medial aspect of the pars interarticularis. Image guidance is helpful for planning the procedure. 


\section{P-108}

\section{Reconstruction of the unstable craniocervical junction and atlanto-axial region with transarticular screws: clinical and radiological outcomes of 51 consecutive cases}

\author{
Benô̂t Goulet, Michael G. Fehlings (Toronto, Ontario)
}

Background: Transarticular screws are an important option for managing atlanto-axial instability, although concerns remain regarding safety and application to other craniocervical pathology. We present our experience with transarticular screw fixation in 51 cases with a mean follow-up of 29 months.

Methods: Indications for surgery included trauma; rheumatoid arthritis; failed fusion; degenerative instability; osteomyelitis and os odontoidum. A C1-C2 fusion alone was performed in 38 cases; transarticular screws were also used as part of an occipitocervical or a $\mathrm{C} 1$ to $\mathrm{C} 3$ cervical reconstruction. An Image Guided System was used in 16 patients. Lateral fluoroscopy was used in all cases.

Results: Four patients had a single $\mathrm{C} 1-\mathrm{C} 2$ screw placed due to anatomical constraints. There were no perioperative new neurological deficits or vertebral artery injuries. On flexion and extension films all patients had good stability and 19 patients had definite solid union. Post operative CT was obtained in 23 cases and screw position was classified using a new system.

Conclusion: Transarticular screws represent an important technique to stabilise the $\mathrm{C} 1-\mathrm{C} 2$ joint and in experienced hands can be placed with minimal risk. This technique should be considered the method of choice for rheumatoid instability and for revision of failed fusions.

\section{P-109}

\section{Risk factors associated with iatrogenic cervical nerve root injury during spine surgery}

\section{D.A. Houlden, D.W. Rowed, M.L. Schwartz, M. Fazl,} R. Midha, J.A. Finkelstein, L.M. Burkholder (Toronto, Ontario)

Purpose: To elucidate risk factors for iatrogenic cervical nerve root injury during cervical spine surgery.

Methods: Mechanically evoked electromyography (EMG) was obtained from muscles supplied by cervical nerve roots most likely at risk during surgery. EMG discharges were classified as "unsustained" or "sustained" (>2 seconds). Pre- and postoperative neurological examinations were performed by an individual blind to the surgical approach and EMG results.

Results: Fifty-three patients were studied (45 with preoperative neurological deficits). Forty-one had cervical spine surgery by an anterior approach, and 12 by a posterior one. Six patients suffered iatrogenic nerve root injury and all six had "sustained" intraoperative EMG. Curiously, five of those had surgery by a posterior approach, and four had pre-operative radiculopathy. All iatrogenic nerve root injuries were in the C5 or C6 distributions except one. Ten patients had "sustained" EMG without new deficits, but only one of those had surgery by a posterior approach and only one had pre-operative radiculopathy. No patient with "unsustained" $(n=6)$ or absent EMG $(n=31)$ had iatrogenic nerve root injury.
Conclusions: Risk factors associated with iatrogenic cervical nerve root injury were posterior surgical approach and preoperative radiculopathy, combined with "sustained" intraoperative EMG. The C5/6 nerve roots were most vulnerable.

\section{P-110}

Pro-cysteine compound decreases acute inflammatory responses and promotes retention of function after spinal cord injury

H. Kamencic, M. Kelly, E. Matshes, R. Griebel, P. Paterson, B. Juurlink (Saskatoon, Saskatchewan), A. Lyon (Calgary, Alberta)

Background: We tested the hypothesis that maintenance of spinal cord glutathione (GSH) will prevent oxidative stress, a major driving force in the inflammatory process that has been implicated in promoting secondary damage.

Methods: Compression trauma (50 g aneurysm clip) at T6 was applied to adult rat followed by intraperitoneal administration of L-2-oxo-thiazolidine-4-carboxylate (OTC) or saline vehicle. Immunohistochemistry was performed for NFkB, inducible nitric oxide synthase (iNOS), intracellular adhesion molecule-1 (ICAM1) and complement receptor C3 (OX-42).

Results: By administering OTC we promoted GSH synthesis and decreased the development of oxidative stress following spinal cord trauma. OTC-treated animals showed better preservation of white matter which resulted in better functional recovery in all tests performed (BBB score, Tarlov score and inclined plane). Inflammatory responses were also reduced after OTC administration. The number of NFkB positive cells were reduced by $70 \%$, iNOS by $70 \%$ ICAM 1 by $40 \%$ and OX-42 by $35 \%$ in comparison to vehicle controls.

Conclusion: Trauma to the spinal cord results in rapid onset of oxidative stress. Maintenance of GSH by OTC administration prevents much of the tissue damage and promotes better functional recovery. Decreasing oxidative stress following spinal injury decreased activation of $\mathrm{NFkB}$ and this correlated with decreased pro-inflammatory gene expression.

\section{P-111}

\section{3-D cone-beam CT of the spine: neurosurgical applications}

R. L. Sahjpaul, D.W. Holdsworth, A.J. Fox, S.P. Lownie, D.H. Lee (London, Ontario)

Background: We investigated the application of a prototype 3-D cone-beam computed tomography (CT) system (previously developed for 3-D angiography) to image the spine.

Methods: In four patients with spinal pathology $130 \mathrm{x}$-ray projections ( $90 \mathrm{kVp}, 55 \mathrm{mAs}$ total) were obtained in 4.4 seconds by single-plane $\mathrm{C}$-arm rotation at 45 degrees per second around the patient. Cone-beam CTreconstruction algorithms produced a $400 \times 400 \times 400$ image volume with isotropic $0.55 \mathrm{~mm}$ voxel spacing. Study parameters included spatial resolution, noise, artifact, and patient dose.

Results: CT reconstruction of the projection data produced a $16 \times 16 \times 16 \mathrm{~cm}$ volume image of the spine. Image noise was \pm 130 
Hounsefield units in background tissue. Patient effective dose was less than $0.1 \mathrm{mSv}$ per volume acquisition (comparable to a C-spine series and less than that of a spiral CT volume acquisition). No significant artifacts were observed near spinal instrumentation (titanium alloy).

Conclusion: 3-D CT imaging of the spine using a C-arm based $\mathrm{x}$-ray imaging system is feasible, yielding high-resolution CT data without compromising standard features (digital radiography and fluoroscopy at prescribed view angles). Advantages include rapid acquisition (4.4 seconds), isotropic spatial resolution, reduced artifact, and low patient dose. Future applications may include frameless stereotactic image-guided surgery.

\section{P-112}

\section{The sodium channel blocker riluzole improves behavioral and neuroanatomical recovery after traumatic spinal cord injury}

\section{Gwen Schwartz, Michael G. Fehlings (Toronto, Ontario)}

Background: Perturbation of voltage-sensitive $\mathrm{Na}^{+}$channel activity may mediate an accumulation of intracellular $\mathrm{Na}^{+}$ associated with the degeneration of neuronal tissue and myelinated axons following traumatic spinal cord injury (SCI). Although therapeutic $\mathrm{Na}^{+}$channel blockers demonstrate neuroprotective effectiveness in degenerative CNS disorders their long-term neuroprotective and functional restorative potential has yet to be determined.

Methods: In the present study, adult rats were randomly and blindly assigned to receive an intraperitoneal injection of either riluzole $(5 \mathrm{mg} / \mathrm{kg})$; phenytoin $(30 \mathrm{mg} / \mathrm{kg}) ; \mathrm{CNS} 5546 \mathrm{~A}$, a novel $\mathrm{Na}+$ channel blocker $(15 \mathrm{mg} / \mathrm{kg})$; or $2-\mathrm{HP} \beta \mathrm{CD}$ as a control $(5$ $\mathrm{mg} / \mathrm{kg}$ ), 15 minutes after a $53 \mathrm{~g}$ clip compression SCI at C7/T1. Clinical neurological recovery of motor behavior was assessed weekly, for six weeks, with the Basso, Beattie, and Bresnahan locomotor rating scale (BBB) and inclined plane (IP) techniques. Seven weeks post-injury the preservation of residual spinal cord tissue and the integrity of supraspinal nuclei associated with descending motor fibers were evaluated with digital morphometry and fluorescent-retrograde tracing, respectively.

Results: Functional recovery $[\mathrm{BBB}(\mathrm{p}=0.0001)$ and IP $(p<0.0001)]$, preservation of residual tissue $(p<0.0001)$, and the integrity of descending rubrospinal tract axons $(p<0.02)$ were significantly effected by drug treatment (ANOVA). Riluzole conferred the greatest neuroprotective effect on all outcome measures $(p<0.05$; post hoc SNK) compared to the other treatments.

Conclusions: Riluzole is neuroprotective after in vivo SCI and is associated with enhanced long-term functional and neuroanatomical recovery.

\section{P-113}

\section{Transplanted subependymal stem cells improve functional neurological recovery after traumatic spinal cord injury}

Gwen Schwartz, Scellig S. Stone, Cindi M. Morshead, Linda Lee, Derek van der Kooy, Charles H. Tator (Toronto, Ontario)

Background: Clonally derived subependymal neurospheres from the adult mouse forebrain exhibit stem cell (sc) properties including a capacity for self-renewal and differentiation along neuronal and glial lineages. Transplantation of subependymal scs into an injured spinal cord may mediate functional neurological recovery by stimulating repair or regeneration of damaged or lost tissue.

Methods: Subependymal forebrain scs, derived from ROSA26 mice expressing the LacZ gene that codes for $\beta$ galactosidase, were stereotaxically transplanted into the spinal cord at T3-4 after moderate clip compression injury at the same site. Female CD1 mice received transplants immediately, three, or seven days post-injury. Functional restorative capacity of transplanted cells were assessed with behavioural recovery profiles generated seven days after transplantation and compared with seven day, post-injury profiles from nontransplanted mice. PCR amplification of the LacZ gene and immunohistochemical analysis of the injury site verified the presence and expression of transplanted scs in injured tissue.

Results: Strong amplification signals for LacZ in genomic DNA isolated from transplanted animals were detected and clusters of rabbit anti- $\beta$-galactosidase positive cells were found adjacent to the intermediolateral injection site. Functional recovery scores from the inclined plane technique were significantly improved in transplanted mice ( $\mathrm{p}=0.007$; ANOVA) as compared to nontransplanted controls ( $\mathrm{p}<$ than 0.05 ; post-hoc Bonferroni). Transplanted mice also exhibited a trend toward improved open field locomotor ability as assessed with the Basso, Beattie, and Bresnahan Rating Scale ( $\mathrm{p}=0.08$; ANOVA).

Conclusions: Transplantation of adult forebrain stem cells into an injured spinal cord is a viable therapeutic strategy resulting in functional neurological improvements and having important implications for neural tissue repair and preservation following trauma.

\section{P-114}

A spinal cord anastomosis surgical repair strategy results in functional and histological improvement following complete spinal cord transection in rats

E.C. Tsai, K.H. Marshall, S.W. Hwang, R. van Bendegem, C. H. Tator (Toronto, Ontario)

Background: We examined the functional and histological results of two promising, experimental spinal cord repair strategies: spinal cord anastomosis (SCA) and peripheral nerve graft repair (PNGR).

Methods: Adult, female Sprague-Dawley rats underwent complete cord transection at T8 and then either SCA $(n=7)$ or 
PNGR ( $n=6)$. Both groups had spinal stabilization, and a combination of minced peripheral nerve and fibroblast growth factor 1 administered in fibrin glue, while the control group $(n=7)$ had transection without repair. Functional assessment utilized the Basso, Beattie, and Bresnahan (BBB) score, and histological assessment included axonal tracers (DiI and FG) and immunohistochemical procedures to show regenerative and inhibitory features after 15 weeks survival.

Results: Both repair strategies produced a small amount of regeneration. Both repair groups had higher BBB scores $(\mathrm{p}=0.003)$ and more axonal regeneration than the control group. The SCAgroup had the highest BBB scores and the most axonal regeneration. Despite the presence of several neurite inhibitors at the spinal cord repair site, there was evidence of axonal regeneration.

Conclusions: Both SCA and PNGR produced some functional improvement and axonal regeneration compared to control, although SCA was superior. The extent of spinal cord repair was minimal and efforts to improve the recovery are ongoing.

\section{GENERAL NEUROSURGERY}

\section{P-115}

\section{Error in neurosurgery: a prospective pilot study}

\section{Bernstein, E. Massicotte, E. Etchells (Toronto, Ontario)}

Background: Error in medicine and surgery is becoming more widely recognized as a common cause of patient morbidity and mortality. The authors recently initiated a prospective study to capture all errors, and assess their preventability and their impact.

Methods: Consecutive patients of one neurosurgeon undergoing surgery during elective operating hours were closely monitored by the surgeon for error in the pre-operative, intraoperative, and postoperative period. An independent observer was not used.

Results: There were 128 consecutive cases during the pilot study period of five months. In $73 \%$ of patients, at least one error was detected and the total number was 221 (average 1.7 per case). Most were minor, but 36 (16.3\%) were considered major. There were $12.5 \%$ complications (14 morbidities and two mortalities) in the 128 patients, two of which resulted from an error. Examples of typical errors and an attempt at classification will be presented.

Conclusions: Discovery and disclosure of error is necessary to create a culture conducive to optimal resident education, health care professional self-improvement, best patient care, and best patient-physician relationships. We are planning a prospective study with grant-funded independent observers which will improve the validity and reliability of error data.

\section{P-116}

The availability and accessibility of internet resources to supplement injury prevention education

T. Sibbald, C. Rodriguez, D. Isaac, M. Cusimano (Toronto, Ontario)

Background: The premise of many injury prevention initiatives is that the number of injuries can be reduced through increased awareness and education. The purpose of this study was to determine the availability, accessibility and quality of free Internet resources related to injury prevention.

Methods: An extensive Internet search was conducted using 15 search engines. Fifty-four searches were performed using search strings including but not exclusive to: sports, bicycle, promotion, information, Ride Safe, kids, safety, child, playground, cycling, teach, brain, vehicular, water, anatomy, biology, seatbelt, spinal cord, bus, car, Think First, and brochure.

Results: Sixteen Internet resources were identified and classified into the six categories: brain and spinal cord $(n=1)$, vehicular safety $(n=2)$, bicycle safety $(n=5)$, violence $(n=1)$, sports and recreational safety $(n=1)$, water safety $(n=2)$, and those covering multiple safety areas $(n=4)$. Of the 16 sources, seven are intended for children, six are for guardians, and three are for teachers. Nine of the 16 resources have been recently updated. Thirteen of these resources are sponsored by public institutions while the remainder was privately sponsored.

Conclusion: Internet resources related to the prevention of injuries in youth exist and should be accessed by professionals working in this field.

\section{P-117}

\section{Cerebrospinal fluid rhinorrhea creating a "natural shunt" in a patient with a colloid cyst of the third ventricle and empty sella}

\section{Edwards, J. Wells (Hamilton, Ontario)}

Background: Cerebrospinal fluid (CSF) rhinorrhea creating a "natural shunt" is a rare but important symptom. If not recognized, it can delay recognition of life threatening intracranial pathology. We report a patient whose colloid cyst was associated with CSF rhinorrhea and empty sella in addition to more classical symptoms of colloid cyst. Diagnosis was delayed for months because of misinterpretation of his symptoms. The CSF leak stopped after removal of his tumour. Imaging clearly showed the CSF fistula from a temporal horn into the sphenoid sinus.

Methods: An extensive literature search was performed using medically related search engines to find articles that had the greatest relevance to colloid cysts, CSF rhinorrhea, empty sella, and any relationship between these phenomena.

Results: We found a plethora of literature detailing the various presentations of patients with colloid cysts, a few reports of patients with concomitant CSF rhinorrhea and colloid cysts, several descriptions of associated empty sella and CSF rhinorrhea, but no reports of colloid cysts, CSF rhinorrhea, and empty sella as concomitant conditions in the same patient.

Conclusions: The natural history of colloid cysts is uncertain, 
and delayed diagnosis due to failure of symptom recognition or atypical presentation may lead to disability or death.

\section{P-118}

\section{Fibrinolytic treatment for intraventricular hemorrhage}

\section{J. Max Findlay (Edmonton, Alberta)}

Background: Intraventricular hemorrhage (IVH) resulting from cerebral aneurysms, vascular malformations or primary parenchymal bleeding is a serious clinical problem when clots distend the ventricular system, compress the adjacent brain, or obstruct cerebrospinal fluid (CSF) flow causing hydrocephalus and elevated intracranial pressure (ICP). In combination with external ventricular drainage (EVD), intraventricular fibrinolysis (IVF) is a promising treatment for selected patients with IVH.

Methods: Eligibility criteria for IVF include: 1. Large IVHs that expand or occlude the ventricular system; 2 . Elevated ICPat the time of EVD insertion or afterwards; 3. Neurological condition compatible with survival; 4. Underlying cause of hemorrhage repaired or unlikely to rebleed. The treatment dose of rt-PA is $4 \mathrm{mg}$, followed by CSF drainage against a 2-cm pressure gradient. Additional rt-PA is given daily as required, with the goal to open ventricular pathways (assessed by daily CT scanning) and maintain catheter patency.

Results: The first patient ever to receive intraventricular recombinant tissue plasminogen activator (rt-PA) for IVH was treated by us in 1990, and since then we have treated an additional 35 patients. The etiology for IVH was aneurysm in 22 $(61 \%), A V M$ in four $(11 \%)$, hypertension or idiopathic in 8 $(22 \%)$ and ventricular catheter insertion in two (6\%). Most patients $(88 \%)$ required one or two injections of rt-PA to establish patency of the ventricular system within three days. Serious parenchymal hemorrhage occurred in one patient with concomitant thrombocytopenia, and bacterial ventriculitis occurred in two others. Twelve (33\%) patients have gone on to require ventriculo-peritoneal shunting, and 27 (75\%) have made good to moderate recoveries (Glasgow Outcome Scale).

Conclusions: Intraventricular fibrinolysis promotes rapid IVH clearance, facilitates ICP management, maintains ventriculostomy patency, and is relatively safe. A randomized trial to determine its effect on patient outcome is proposed.

\section{P-119}

\section{Management of chronic subdural hematoma}

I.G. Fleetwood (Stanford, California, USA), S. Johnson, A. Abdoh, D. Fewer (Winnipeg, Manitoba)

Background: Patients with chronic subdural hematoma $(\mathrm{cSDH})$ are commonly encountered in clinical practice. Numerous treatment modalities have been described, but none has proven superior with respect to clinical or radiographic outcome.

Methods: A five-year retrospective analysis of patients treated at a single institution for $\mathrm{cSDH}$ was performed. Outcome measures included Glasgow Outcome Score and rate of treatment failure. Radiographic parameters measured included planimetric hematoma volume (PHV), midline shift, and
Destandau's index. PHV was compared to the ABC/2 method of volume calculation. The use of irrigation or a subdural drain was studied to determine predictive value for treatment success. Medical treatment with dexamethasone was compared to various surgical treatments.

Results: Eighty-two patients were included. The transient complication rate from steroid use was low (4.3\%). Drain use $(\mathrm{p}=0.07)$ and a large PHV $(\mathrm{p}=0.025)$ correlated with successful outcome. PHV correlated significantly $(\mathrm{r}=0.9014, \mathrm{p}=0.0001)$ with volume calculated by the $\mathrm{ABC} / 2$ method. There was no difference in outcome or treatment failure rate related to the medical or surgical treatment method used.

Conclusion: This study validates the $\mathrm{ABC} / 2$ method of $\mathrm{cSDH}$ volume calculation. Optimal treatment for these patients remains controversial. The risks with steroid use are low. A randomized prospective multicentre trial is needed.

\section{P-120}

The successful integration of the brain lab image-guided system and a mobile 1.5-Tesla intra-operative magnetic resonance imaging system

\section{M.G. Hamilton, S.T. Myles, T. Kaibara, G.R. Sutherland (Calgary, Alberta)}

Introduction: We have previously reported the development of a high field (1.5-tesla) magnetic resonance imaging (MRI) system for use in the neurosurgical operating room where standard neurosurgical tools (microscope, endoscopes, ultrasonic aspirator, bipolar coagulation, etc.) can be utilized. The magnet is moved to and from the neurosurgical field utilizing overhead crane technology. High-definition $\mathrm{T}_{1}{ }^{-}$and/or $\mathrm{T}_{2}$-weighted images or MR angiography can be acquired at various stages of the surgical procedure.

Methods: This mobile MRI system has been used during 170 neurosurgical procedures. We report the successful integration of the BrainLAB ${ }^{\circledR}$ Image-Guided system into the intra-operative MRI system. Initial preoperative MR images are acquired with the patient positioned in an MRI-compatible 3-pin head holder while utilizing a unique fiducial system. Direct data transfer is completed and patient registration occurs utilizing the VectorVision2 BrainLAB ${ }^{\circledR}$ system. The BrainLAB ${ }^{\circledR}$ registration process takes less than 10 minutes. Patients can be re-registered after acquiring new intra-operative MR images.

Results: We have utilized this system in 25 patients undergoing cranial or spinal neurosurgical procedures since December 1999. The last 15 patients had preoperative or, when undertaken, intra-operative registration with an average accuracy of less than $2.0 \mathrm{~mm}$.

Discussion: The integration of an image-guided system into a high-field intra-operative MRI system produces a significant advantage for the neurosurgeon during complex intracranial or spinal surgery. This provides the neurosurgeon with all of the initial benefits associated with use of an image-guided system, yet allows re-registration after new MR images establish both the extent of surgical progress and brain shift. 


\section{P-121}

\section{A structured analysis of methodology in the clinical neurosurgical literature}

\section{B.S. Jhawar (London, Ontario), M.D. Taylor(Toronto, Ontario)}

Introduction: During the last decade, medical decisionmaking has shifted its emphasis away from expert opinion towards evidence-based practices. The objective of this study was to see if clinical methodology used in the neurosurgical literature has improved over time and to determine potential predictors of improved methodology.

Methods: At five-year intervals between 1980 and 2000, all articles published in three neurosurgical journals were reviewed. Clinical articles were categorized by study type, temporal design, blinded comparison, use of gold standards and randomization. An hierarchical methodology scale was developed to rank clinical methodology for diagnostic and therapeutic papers. This scale was used to model potential predictors of improved methodology using linear regression.

Results: Clinical papers represented $63 \%$ of research papers $(\mathrm{n}=4519)$, with little change over the study period. Among clinical papers, case reports $(88 \%)$ defined as having a sample size of less than 11 were most prevalent, followed by case series (7\%), and comparative studies (4\%). There was a steady increase in the proportion of prospective studies as well as randomized studies. Using the methodology scales, linear regression revealed that higher methodological quality was associated with biostatistician and/or epidemiologist involvement and grant support. There was an increase in the number of authors per paper over time, but this did not predict improved methodology scores.

Conclusion: There is some evidence of improved research methodology in clinical neurosurgical research over the past two decades. However, the proportion of retrospective, uncontrolled, nonrandomized studies remains high.

\section{P-122}

\section{Palm $^{\text {TM }}$ computing applications for the clinical neurosciences}

\section{B.S. Jhawar (London, Ontario)}

It is anticipated that personal digital assistant (PDA) technology will become as pervasive as email and cellular phones in the management of medical information. To date, most software has been designed for the general physician, and there are few programs that are geared towards the needs of the clinical neurologist or neurosurgeon.

This paper discusses the role of PDA technology in medical information management. Three programs are presented that were developed by the author to facilitate clinical problem solving, information access and neuroscience education. The software was written in $\mathrm{C}$ programming language using the Palm ${ }^{\mathrm{TM}} 3.5$ operating system.

1) AVM Re-Hemorrhage Predictor is a program that allows the neurosurgeon to assess individual patient risk for AVM rehemorrhage based on a simple probability model. The software calculates the cumulative probability of rehemorrhage based on a patient's age and expected survival time.

2) AVM Reference Database is a program that allows the user to access reference-based medical information. The menudriven interface allows rapid access to information in a fast and efficient manner. This software also allows the calculation of patient specific information such as the Spetzler-Martin grade.

3) Peripheral Nerve Analyzer is a program that allows the user to rapidly localize peripheral nerve injuries. The software, although intended for the primary care physician can also be used as a teaching tool for residents and medical students.

\section{P-123}

Televideo neurosurgical conferencing in Atlantic Canada I. Mendez (Halifax, Nova Scotia), S. Walling (Saint John, New Brunswick), P. Gorman (Moncton, New Brunswick), M. Maharaj (Sydney, Nova Scotia)

Introduction: A regional televideo conferencing program has been established in four centres in Atlantic Canada (Halifax, Saint John, Moncton and Sydney). The televideo conference infrastructure is well established in these four centres. The technology allows for reliable transmission of images, audio, radiographs, intraoperative video and computer data. We describe here our experience with this program which has been in place for 18 months.

Methods: The number of televideo conferences, participants and cases presented were recorded for an 18 month period.

Results: Sixteen televideo conferences were completed in 18 months. An average of 12 consultants and six residents attended each session. Fifty-four neurosurgical cases were presented, 27 cases were spinal, 21 cranial and six pediatric.

Conclusion: The experience of televideo conference in neurosurgery in Atlantic Canada has been extremely positive. The educational value has been high for both the academic and nonacademic centres. Particularly useful has been the opportunity of regular consultation in difficult neurosurgical cases among the four centres. Furthermore, this program has enhanced the communication among the neurosurgeons in Atlantic Canada and has promoted regional collaboration and integration.

\section{P-124}

\section{A comparative study of delta vs standard shunts in adults}

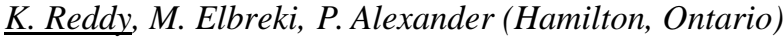

Background: Hydrocephalus is treated commonly by various shunt procedures, all associated with complications. Delta valve design is thought to allow for better function irrespective of body position and cerebrospinal fluid (CSF) flow. The authors prospectively compare delta valves with standard shunts, specifically with regard to complication rates.

Methods: Delta valves and standard shunts were used in a prospective cohort of patients using the alternating method of 
randomization. Patients were followed for a period of time no less than one year. Data were collected prospectively. Interim analysis was performed on 71 patients, of whom 35 had standard shunts and 36 had delta valves. The specific end points were malfunction rate, infection rate and other complications. Statistical tests used were student t-test for continuous data, and Chi square analysis for nonparametric data.

Results: No differences were found in the demographic characteristics between the two groups. Of the 71 patients analyzed, 13 were lost to follow-up. There was no statistical difference between groups in terms of complication rate, infection rate and malfunction rate in general.

Conclusion: The authors conclude from the interim analysis that there is no significant difference between the delta valve and standard shunt systems in terms of complication rate, infection rate and the general malfunction rate. The authors plan to complete the analysis, but given the methodological inadequacies in this study, a randomized controlled trial would likely yield a more generalizable result.

\section{P-125}

\section{Patient satisfaction with neurosurgical care}

\section{R. Sahjpaul, D. Bisnaire, P. Doyle-Pettypiece (London,} Ontario)

Background: Patient satisfaction is increasingly reported as an outcome in evaluation of care provided. The Picker/ Commonwealth Program for Patient-Centered Care identified seven broad dimensions of care that most affect patients' experiences in hospital. They include: respect for values, coordination of care, communication, physical care, emotional support, involvement of family and transition from one locus of care to another.

Methods: We distributed 40 questionnaires to adult patients, discharged alive, over a 20 day period from an academic neurosurgery service. Thirty-one completed questionnaires were returned. All of the surveyed patients underwent surgery during hospitalization (spinal $n=12$, intracranial $n=7$, carotid endarterectomy $n=5$, unknown $n=5$, VP shunt $n=1$, functional procedure $\mathrm{n}=1$ ).

Results: Eighteen patients indicated no negative experiences during their hospital stay. Seven patients reported concerns about incomplete discharge instructions, four noted that medical staff were unable to meet with them or their families. One patient felt that "sometimes" physicians spoke as though he was not there. Other concerns included the physical environment, diet, nursing care and a feeling of lack of control.

Conclusions: Results of the questionnaire have been shared with medical staff. Written information regarding access to team members and surgery specific discharge instruction sheets have been developed.
P-126

\section{An automatic volume based image to frame coordinate transformation for MRI stereotactic surgical planning}

Y.P. Starreveld, K.W. Finnis, T.M. Peters (London, Ontario)

Background: Frame-based stereotaxy is the method of choice for deep brain functional procedures where high accuracy, patient immobilization, and rigid instrument fixation are required. Its accuracy is dependent on precise positioning of the frame, minimization of spatial errors intrinsic to the imaging modality used, the mechanical properties of the frame, and the planning computation. The planning computation depends on the accurate selection of fiducial points in the image. Errors in selecting these points propagate through the computation to degrade the final result and are the largest source of application error.

Methods: An algorithm has been developed and implemented on a computer to automatically extract images of Leksell G frame fiducial markers from magnetic resonance (MR) and Xray computed tomography (CT) images. An image to Leksell space transformation is computed volumetrically in less than one second. A validation study was done using a phantom study.

Results: The mean vector error is $0.5 \pm 0.3 \mathrm{~mm}$ for CT and $0.6 \pm 0.3 \mathrm{~mm}$ for MR images.

Conclusions: This novel method of coordinate calculation is more accurate than manual computations. This technique has particular application to MR based stereotaxy where continued monitoring of image acquisition quality is required to prevent loss of accuracy.

\section{TRAUMA}

\section{P-127}

\section{The epidemiology of head injured patients with surgically treated acute subdural and epidural hematomas: a three-year review of tertiary care referrals}

\section{J.M. Tallon, D.B. Clarke, S. Ackroyd (Halifax, Nova Scotia)}

Background: To review the epidemiology of surgically treated post-traumatic epidural (EDH) and subdural hematomas $(\mathrm{SDH})$ referred to tertiary care in the province of Nova Scotia.

Methods: All adult patients, from May 23, 1996 to May 22, 1999, presenting to our provincial tertiary care hospital with post-traumatic EDH and/or SDH, and who were surgically treated, were included. Penetrating trauma cases were excluded, as were patients that presented beyond 24 hours. A total of 275 charts were reviewed and 24 patients met the study criteria. A subsequent blinded, explicit chart review was performed using a standardized data collection form. Descriptive statistics were generated using STATA statistical software.

Results: Thirteen patients (54\%) presented with SDH, eight $(33 \%)$ with EDH and three $(12.5 \%)$ with both. The average age was $42( \pm 20)$ years and $79 \%$ were male. The mechanisms of injury were evenly divided between falls (43\%) and motor vehicle collisions (43\%); alcohol was an associated factor in 10 (42\%) cases. Most patients had at least one co-morbid medical 
condition (substance abuse, 25\%; cardiac, $17 \%$; neurologic, $8 \%$ ). Approximately $70 \%$ were transferred from referring facilities. There were four postoperative deaths (17\%). Six month outcome (GOS) data were available for 16 patients; of these, nine $(56 \%)$ had a GOS $\geq 4$.

Conclusion: The results of this study have implications for neurosurgeons who are involved in the regionalization of health care services, particularly as it relates to further evaluation and development of the provincial emergency medical system.

\section{P-128}

\section{A computerized interactive tool to assess injury-related behavioural intentions in primary school children}

\section{Isaac, I. Kalnins, M.D. Cusimano, E. Mansfield (Toronto,} Ontario)

Background: Although a number of injury prevention education resources for youth exist, these programs require the use of valid and reliable evaluative measures to assess behaviour and outcomes. While interactive computer-based tools that measure safety knowledge among primary school children have been developed, a similar computer-based approach for analysing behavioural intentions is required. Using ethnographic techniques, a scenario-based instrument to evaluate behavioural intentions was developed. The purpose of this work was to create a computerised, cost-effective interactive tool to assess injury related intentions that could be used in large-scale studies.

Methods: Using the Microsoft Visual Basic programming language, a previously developed questionnaire assessing the safety behavioural intentions of primary grade children was digitized. Ten vignettes related to vehicular safety, bicycle safety, violence, sports and recreation, and water safety were developed into a computer readable format.

Results: The HealthScope computer program, through illustrations and the optional narration of text, presents children with scenarios related to safety topics. Once the scenario is presented to the child, he/she is given choices about what he/she would do in the described situation. Based upon the child's chosen response, the child is then asked to select one of five options to explain why he/she made the initial selection between the two choices. The choice and explanation for the choice along with child's name, age and sex are then recorded in a database and then easily imported into readily available software programs.

Conclusions: HealthScope is a program that allows for the rapid collection of data related to a child's behavioural intentions relating to safety. It accomplished this goal in a fun and interactive manner.

\section{P-129}

\section{Curricular mapping of the Think First for Kids program for provincial curricula in Canada}

\section{Rodriguez, J. Patterson, M. Cusimano (Toronto, Ontario)}

Background: The Think First for Kids Program (TFFK) is a comprehensive injury prevention program aimed at promoting healthy behaviour in children. The program teaches important safety lessons in six modules to children in grades 1,2 , and 3 . Every province has clearly defined curricular guidelines in different subject areas. This study evaluated the extent to which the TFFK program covers the learning outcomes and objectives required by provincial education ministries.

Methods: Official documents were collected from each province in all relevant subject areas. A qualitative content analysis of each curriculum determined the correlation between the TFFK program and individual provincial curriculum requirements. The study focused on health and physical education while also examining possible cross-curricular overlap in other subject areas.

Results: TFFK covers many provincial learning objectives related to safety and safe behaviours in the subject areas of health and physical education. The program also reinforces learning objectives in other subject areas, including mathematics, language arts, social studies and science.

Conclusion: TFFK successfully meets several provincial health and physical education curricular requirements related to safety. Given the comprehensive nature of TFFK, it serves as a valuable educational tool that can be easily integrated into already existing provincial curricula across Canada.

\section{P-130}

\section{Changing incidence and patterns of pediatric head injury in Saskatchewan}

M. Kelly, H. Kamencic, N. Muhajarine, R. Griebel

(Saskatoon, Saskatchewan), C. Ekong (Regina,

Saskatchewan)

Background: Pediatric head injury represents a major public health issue. Prevention is recognized as the most effective intervention. To this end, seatbelt and helmet legislation as well as public awareness programs have been introduced over the past decade.

Methods: A case controlled study of head injuries in individuals less than 20 years of age treated at a tertiary referral center in the years 1990 and again in 1999 was performed. Injury severity, incidence, causality, outcome, and patient demographic features were reviewed and statistically analyzed.

Results: A significant decrease in head injuries was documented in this age group over a 10 year span, particularly in mild (GCS <13) head injuries. Seatbelt use significantly increased over the decade, and there was a decrease in both bicycle and sports related injuries. MVA and pedestrian accidents remained common. Trauma outcomes did not change over the decade.

Conclusions: There is a declining incidence of pediatric head injuries in Saskatchewan. While it is difficult to link this directly to any preventive program, a causal link seems possible. 
P-131

Trauma involving head injury results in greater body oxidative stress than those involving spine injury

A. Obayan, R.W. Griebel, R. Keith, B.H.J. Juurlink

(Saskatoon, Saskatchewan)

Background: Our study was aimed at determining whether head trauma resulted in greater overall oxidative stress than spine trauma.

Methods: Oxidative stress was measured by determining extent of erythrocyte glutathione (GSH) depletion and increase in neutrophil counts. Entry criteria for the study included all multiply injured patients at the Royal University Hospital, Saskatoon, Saskatchewan. Patients were subsequently divided into three groups based on the nature of injury: head injury, combined head and body injury, spine and body injury. GSH was measured, using a modification of the Brigelius method, in packed cell samples taken at $0,6,12,24,72 \mathrm{hrs}$ and on day seven.

Results: There was a correlation between severity of injury and the degree of depletion of erythrocyte GSH with maximal correlation seen at 24-30 hrs following admission. Patients with head injury had a more severe depletion in the red cell GSH levels than did spinal injured patients. The neutrophil count in head injured patients was also greater although not significantly so.

Conclusion: Head injury caused a more severe increase in oxidative stress than spinal injury, suggesting antioxidant therapy may be of benefit.

\section{PERIPHERAL NERVE}

\section{P-132}

\section{Neural stem cell transplantation in peripheral nerve injury \\ L. Crevier, N.S. Huq, L.C. Doering, J.R. Bain (Hamilton, Ontario)}

Background: Neural precursor cells (NPC) and neural stem cells (NSC) may be used to help preserve denervated skeletal muscle after peripheral nerve injury. The authors compare the functionality of fetal anterior horn cells (FAH) and cerebellar derived stem cells (C17.2) in a rat peripheral nerve injury model.

Methods: Forty adult female Lewis rats had their right sciatic nerves transected, leaving the left nerve as control. The first group had no intervention. The second group had immediate microsurgical repair. The third group had FAH cells transplanted into the distal stump, and the fourth group had C17.2 cells similarly transplanted. Electromyographic and histologic studies were performed.

Results: Both the FAH and the C17.2 groups demonstrated muscle innervation in eight of the 10 animals. A fetal muscle subgroup was identified in the FAH group, whereas the C17.2 cells seemed to transform the end organ into type II muscle. Axonal filaments were also demonstrated in both these groups.

Conclusions: The neural stem cells transplanted into the distal stump of an injured peripheral nerve demonstrated an ability to re-innervate denervated peripheral muscle as demonstrated by histologic and electrophysiologic studies.

\section{P-133}

\section{Functional outcome of ulnar nerve neurolysis}

\section{Jacques, S. Richard, T. Del Duca (Montreal, Quebec)}

Objective: There are few reports in the literature on the functional outcome of ulnar nerve neurolysis. A retrospective chart review was undertaken to determine the functional outcome post-neurolysis.

Methods: Fifty patients were evaluated pre- and postoperatively with a view to their functional status and limitations. Clinical evaluations, operative reports, and followup assessment were studied.

Results, Conclusions: Major functional improvements are seen post-neurolysis. The final outcome is dependent on several factors, such as the extent of nerve injury seen intraoperatively and the delay between onset of symptoms and surgical intervention. Ulnar neurolysis has a positive functional impact on these patients.

\section{P-134}

\section{Meralgia paresthetica: an update on the surgical management and related controversies}

\section{Jacques, S. Richard, T. Del Duca (Montreal, Quebec)}

Background: Meralgia paresthetica (MP) was initially described by Bernhart in 1895. Roth first coined the term from its Greek origin meros meaning thigh and algos relating to pain. The classic presentation consists of numbness, tingling, pain, and burning sensation along the anterolateral thigh region. Multiple etiologies have been associated with MP, including iatrogenic causes. This report aims to present the clinical features, as well as therapeutic options available in the management of MP.

Methods: Over 200 patients with MP were treated by the senior author. All patients were first managed conservatively. This included optimization of medications, local infiltration with anesthetic and steroid agents, and removal of possible offending sources. Despite these measures, 10 cases ultimately required transection of the lateral femoral cutaneous nerve.

Conclusion: MP is a rare but distressing condition that may result from iatrogenic complications. When first-line conservative treatment fails, surgical alternatives may be beneficial in selected patients. Resection of the lateral femoral cutaneous nerve represents a viable option for this subgroup of patients. Results of the surgical series will be presented in detail along with a review of the literature. 


\section{PEDIATRIC NEUROSURGERY}

\section{P-135}

\section{Congenital glioblastoma multiforme presenting with complex partial seizures}

S.S. Baeesa, H.H. Ibrahim, M.M.S. Jan (Jeddah, Saudi

Arabia)

Background: Congenital brain tumors are very rare accounting for $0.5-1.5 \%$ of all cases of childhood brain tumors. Only 2-9\% of these tumors are accounted for by glioblastoma multiforme (GBM), which has the poorest prognosis with no recorded cases surviving beyond one year. Hydrocephalus, vomiting, failure to thrive, and asymmetric skull growth are the usual presenting features. We present an infant with GBM who presented with complex partial seizures.

Method: Case description and review of the relevant literature.

Results: A three-month old male infant presented with brief episodes of tonic posturing, cyanosis, and staring. He was previously healthy with uncomplicated pregnancy and delivery. Clinical examination was normal apart from a head circumference on the 90th percentile. The seizures were controlled with intravenous phenytoin. Brain MRI revealed 5x6 $\mathrm{cm}$ left temporal lobe lesion with homogenous enhancement and significant mass effect. Left temporal craniotomy and complete resection was performed with no residual tumor seen in the postoperative MRI. Pathological examination was consistent with GBM. The infant recovered well from surgery, but developed frequent partial seizures that were resistant to multiple antiepileptic drugs. Topiramate was prescribed and resulted in complete seizure control. He received chemotherapy and remains neurologically stable with no recurrence at one year of age.

Conclusions: GBM should be considered in the differential diagnosis of infants with congenital intracranial tumors. Infants with GBM may present initially with partial seizures only. The histological and immunochemical features of this undifferentiated tumor are similar to those reported in adults.

\section{P-136}

\section{Acute subdural hematoma in neonates with unrecognized hemophilia $\mathrm{A}$ - a persisting problem}

\section{L.M. Myles, P. Massicotte, J.M. Drake (Toronto, Ontario)}

Background: Hemophilia is a rare disorder, and an uncommon cause of intracranial hemorrhage in neonates. We present two patients with hemophilia A, who presented with massive subdural hemorrhages on day five and day four post partum, following difficult deliveries. Despite preoperative routine coagulation screens, both patients were operated upon without the diagnosis of hemophilia being recognized.

Clinical course: Neither patient had a family history of hemophilia. In the first patient, the activated partial thromboplastin time (APTT) was within the normal range for age. When the patient rebled, a factor VIII level of $10 \%$ established the diagnosis of moderately severe hemophilia A. The patient suffered hemispheric infarction and is severely disabled. In the second patient, the APTTwas slightly prolonged, but initially interpreted as being normal for age (APTT is prolonged in normal neonates compared to older children). Nevertheless fresh frozen plasma was given preoperatively. A factor VII level of $<1 \%$ postoperatively established the diagnosis of severe hemophilia A. The patient made a good recovery.

Conclusion: The combination of a history of a traumatic delivery; absence of a positive family history; clinical crisis produced by a large intracranial clot; normal or slightly prolonged APTT (which may be misinterpreted as being within the normal range for age); can make the diagnosis of hemophilia in this age group very difficult. A high degree of suspicion is required in these cases and it is strongly recommended that Factor VIII assay be performed in all term male babies presenting with intracranial hemorrhage. In urgent circumstances, without Factor VIII levels, prophylactic clotting therapy should be administered during surgery to prevent postoperative bleeding in an undiagnosed hemophiliac.

\section{P-137}

\section{Initial experience with a new programmable valve $\left(\right.$ Strata $\left.^{\circledR}\right)$ for the surgical treatment of hydrocephalus}

\section{M.G. Hamilton (Calgary, Alberta)}

Introduction: The surgical management of hydrocephalus with ventriculoperitoneal shunts remains a difficult endeavor frequently associated with problems of over drainage (e.g. subdural effusions or hematomas) and under drainage (e.g. ineffective treatment). These problems may significantly complicate treatment outcome, and can result in additional surgical procedures. The Strata ${ }^{\circledR}$ valve was introduced in Canada as an adjunct for the treatment of hydrocephalus in July 2000. The Strata ${ }^{\circledR}$ valve allows for five separate permutations of the Delta $^{\circledR}$ valve (Delta $\left.{ }^{\circledR} 0.5,1.0,1.5,2.0,2.5\right)$ with transcutaneous adjustments to valve resistance easily made after implantation. The Delta ${ }^{\circledR}$ valve has "antisiphon" capabilities.

Methods/Results: A total of 18 patients have had a Strata ${ }^{\circledR}$ valve inserted either for the initial treatment of hydrocephalus or at the time of a shunt revision. There were two pediatric patients and 16 adult patients. The indications for utilization of the Strata ${ }^{\circledR}$ valve were not standardized, but were left to the discretion of one of four attending neurosurgeons. The aim of this preliminary assessment was to evaluate ease of use, reliability, and safety. Adjustments to valve resistance were made in $13(72 \%)$ patients, predominately to decrease outflow resistance. All valve adjustments were accomplished without difficulty. One patient eventually had her shunt removed after a third ventriculostomy was performed. No valve malfunction or safety issues were encountered.

Discussion: The Strata ${ }^{\circledR}$ valve offers the capability for noninvasive change in shunt system outflow resistance. This initial experience suggests that this valve could be used with ease and safety during management of hydrocephalus. A prospective, randomized clinical trial to assess efficacy is being developed. 


\section{P-138}

The successful utilization of a mobile 1.5-Tesla intraoperative magnetic resonance imaging system during pediatric neurosurgical procedures

\section{M.G. Hamilton, S.T. Myles, T. Kaibara, G.R. Sutherland (Calgary, Alberta)}

Introduction: We have previously reported the development of a high field (1.5-tesla) magnetic resonance imaging (MRI) system for use in the neurosurgical operating room where standard neurosurgical tools (microscope, endoscopes, ultrasonic aspirator, bipolar coagulation, etc.) can be utilized. The magnet is moved to and from the neurosurgical field utilizing overhead crane technology. High-definition $\mathrm{T}_{1}-$ and/or $\mathrm{T}_{2}$-weighted images or MR angiography can be acquired at various stages of the surgical procedure. A VectorVision2 BrainLAB $^{\circledR}$ Imageguided system is integrated into the MRI system. Patients can be re-registered after acquiring new intra-operative MR images.

Methods/Results: This mobile MRI system has been used during 170 neurosurgical procedures.

Between November 1998 and January 2001, 22 pediatric patients underwent surgery utilizing the intraoperative MRI system. The average age was 11.6 years (range 16 months- 17 years) with 12 male and 10 female patients. Patient diagnosis included tumor $(n=15)$, epilepsy $(n=4), \operatorname{AVM}(n=1)$, cavernous malformation $(n=1)$, and spinal cord tumor $(n=1)$. Immediate preoperative and intraoperative MR imaging was completed in all patients. The BrainLAB ${ }^{\circledR}$ Image-guided system was utilized in eight patients.

Discussion: The use of a high-field intra-operative MRI system provides a potential significant advantage for the pediatric neurosurgeon during complex intra-cranial or spinal surgery. Immediate feedback concerning the extent of tumor resection, and the availability of an integrated frameless stereotaxy system that can be used with both preoperative and intra-operative images can greatly enhance surgical effectiveness and safety. Greater clinical experience will, however, be required to validate this initial impression.

\section{P-139}

\section{Reduction of rates of allogeneic blood transfusion in operations for correction of sagittal synostosis}

\section{S. Hentschel, P. Steinbok, D. Cochrane (Vancouver, British Columbia), J. Kestle (Salt Lake City, Utah, USA)}

As public concern about the risks of blood transfusions increased in the mid 1990s, avoidance of transfusions became a goal of surgery for sagittal synostosis. This study was performed to confirm a hypothesized reduction in transfusion rates in recent years and to identify factors associated with need for transfusion.

Sagittal synostosis operations in children between 1986 and 1999 were reviewed retrospectively. There were 118 patients, with median age at operation of 4.2 months. Patients underwent a minimum of vertex strip craniectomy and parietal craniectomies. Logistic regression revealed that more extensive surgery, older age, lower preoperative hemoglobin, longer operative time, and surgery before 1996 were associated with increased likelihood of transfusion. $42 \%$ (95\% CI 31\% - 52\%) before 1996 and 11\% (95\% CI 0\% - 23\%) since 1996 received blood. Techniques to minimize blood loss included: use of the Colorado needle for scalp incision, Midas Rex craniotome for cranial cuts, and microfibrillar collagen. Postoperative hemoglobin was allowed to fall to $60 \mathrm{~g} / \mathrm{l}$ if the child was stable hemodynamically, before giving blood. There were no cardiovascular or wound healing complications, and no repeat surgeries for cosmetic reasons.

Low blood transfusion rates were achieved using simple intraoperative techniques and by accepting a low postoperative hemoglobin.

\section{P-140}

\section{Repeat cerebrospinal fluid shunt infection in children}

A.V. Kulkarni, D. Rabin, M. Lamberti-Pasculli, J.M. Drake (Toronto, Ontario)

Introduction: In this study, we investigated the treatment of cerebrospinal fluid (CSF) shunt infection and the risk factors for repeat shunt infection (RSI) in a cohort of children treated at the Hospital for Sick Children, Toronto.

Methods: Between 1996 and 2000, a total of 50 children were identified with shunt infection (mean age 5.9 years). The medical records of these children were reviewed to identify cases of RSI within six months of the initial shunt infection.

Results: Of the 50 initial shunt infections, the infecting organisms were: coagulase-negative Staph. (44\%), Staph. aureus $(34 \%)$, and others $(22 \%)$. The initial mode of treatment of the shunt infection was external ventricular drainage (EVD) with removal of shunt apparatus (62\%) or, in cases in which the ventricles were too small to place an external drain, externalization of the shunt (38\%). The mean number of days of external CSF drainage (either EVD or externalized shunt) was 10.4 days. Ten patients (20\%) developed RSI. The actuarial risk of RSI plateaued after 90 days at $25.0 \%$. The following variables were tested as risk factors for RSI using survival analysis, although none reached statistical significance: age $(\mathrm{p}=0.48)$, etiology of hydrocephalus $(\mathrm{p}=0.64)$, initial organism $(\mathrm{p}=0.24)$, type of surgical treatment of the initial shunt infection $(\mathrm{p}=0.54)$, and number of days of CSF drainage $(\mathrm{p}=0.44)$.

Conclusions: The risk for RSI is substantial and greater effort needs to be directed towards understanding risk factors. Such studies will need greater sample size in order to obtain sufficient statistical power.

\section{P-141}

\section{Focal malignant midbrain tumours in young children:} report of two cases

T.G. Mainprize, S. Baruchel, M.D. Taylor, S. Blaser,

\section{L.E. Becker, P.B. Dirks (Toronto, Ontario)}

Background: Focal midbrain tumours are typically low grade neoplasms of childhood. We report two cases of malignant focal tumours involving the ventral midbrain in young children that rapidly spread throughout the CNS. 
Methods: A review of the clinical course of the two children was performed.

Results: Both children (age two years and 14 months) presented with third nerve palsies and the tumours were located in the ventral midbrain. On MRI, the lesions were solid and uniformly enhancing without a well-circumscribed margin. Because of the lesions' location and their presumed low grade nature, a decision was made to follow the children closely. Both lesions changed in size within two months of original diagnosis and one rapidly disseminated. Open biopsies were performed and the diagnosis in one was malignant rhabdoid tumour and the other was a completely undifferentiated malignant tumour. The latter lesion demonstrated features suggestive of a rhabdoid tumour but a definitive diagnosis of this tumour type could not be made. Both patients were treated with chemotherapy but continued to rapidly progress and they died five and 11 months post-diagnosis.

Conclusions: The majority of focal midbrain tumours are low grade. Malignant tumours can occur in this location and suspicion should be raised in very young children with focal masses with atypical imaging features. These lesions may be rhabdoid tumours and consideration should be made for closer follow-up and biopsy.

\section{P-142}

\section{Comparison of motor outcomes after selective dorsal rhizotomy with and without preoperative intensive physiotherapy in children with spastic diplegic cerebral palsy}

\section{P. Steinbok, K. McLeod (Vancouver, British Columbia)}

A previous randomized clinical trial compared selective dorsal rhizotomy (SDR) plus postoperative intensive physiotherapy (Group 1) with intensive physiotherapy alone (Group 2) for children with spastic diplegic cerebral palsy. At the end of this trial, all patients in Group 1 had a SDR, followed by further intensive physiotherapy. This study was performed to determine if the additional intensive physiotherapy before SDR, as occurred in Group 2, improved long-term motor outcome.

Outcomes were compared in two groups: Group $1-$ no intensive physiotherapy before SDR and Group 2 - intensive physiotherapy before SDR. The primary outcome measure was the GMFM. Lower limb spasticity, range and strength were secondary outcome measures. Baseline assessments had been done for the prior clinical trial. For this study, patients were reassessed by physiotherapists blinded to the treatment group.

Thirteen children in each group were studied at a mean follow-up of 53 months. The mean improvement in GMFM was 10.0 in Group 1 and 10.4 in Group $2(\mathrm{p}=0.6)$. Improvements in spasticity and range were similar in the 2 groups. There was no change in muscle strength in either group.

It was concluded that additional intensive physiotherapy before SDR did not improve motor outcomes.
P-143

Persistent seizure disorders secondary to residual/recurrent dysembryoplastic neuroepithelial tumours

Michael D. Taylor, Laurence Becker, James T. Rutka, Robin Humphreys, Todd G. Mainprize, Peter Dirks (Toronto, Ontario)

Background: Dysembryoplastic neuroepithelial tumours (DNET) are low grade, supratentorial, cortical based lesions that usually present with partial complex seizures in children or young adults. Current literature on these lesions suggests that they are extremely benign, with little or no growth potential and can be treated with partial surgical resection resulting in satisfactory remission of seizures.

Methods: We reviewed the Hospital For Sick Children experience with DNETs.

Results: 4/21 children with DNET experienced persistent or recurrent seizure disorders after surgical resection, contradicting what has been published in the literature. These four patients presented on average three years after their primary surgery with recurrent seizures. Median age at initial presentation in children with recurrent tumours was seven years. While DNETs have been thought to be stable lesions over time, we present evidence of continued DNET growth over time on serial imaging. Recurrent DNETs showed classic features on histology such as the specific glioneuronal element and there was no evidence of malignant degeneration in any of the recurrent tumours.

Conclusions: These findings suggest that DNETs are indeed neoplastic lesions and not hamartomas, that gross total resection should be attempted when safe, and further suggests that children should be followed long-term after removal of a DNET to rule out recurrence.

\section{P-144}

Spontaneous hydrocephalus and leptomeningeal leukemia in $\mathrm{p53}^{-/-}$RAG-2 ${ }^{-/-}$SCID triple mutant mice: a novel model of leptomeningeal leukemia

Michael D. Taylor, Rebecca A. Gladdy, Christine J. Williams, Jana Karaskova, Jeremy A. Squire, James T. Rutka, Cythia J. Guidos, Jayne S. Danska

(Toronto, Ontario)

Background: Spread of systemic leukemia to the CNS occurs almost exclusively in the leptomeninges, not the brain parenchyma. Prophylaxis against leptomeningeal leukemia (craniospinal radiation) has serious side effects including diminished cognition and secondary neoplasia. Mice mutant for p53 and DNA-PK (SCID mutation) have impaired DNAdamage repair and a high incidence of systemic leukemia. RAG genes are involved in cleaving DNAduring lymphocyte development.

Methods: We bred $\mathrm{p} 53^{-/}$RAG- ${ }^{-/-}$SCID mice and analyzed the phenotype.

Results: Whereas $\mathrm{p} 53^{-/}$SCID mice die of systemic leukemia, p53 $3^{-/}$RAG-2 ${ }^{-/}$SCID mice develop a stereotypical syndrome of domed-head, decreased feeding and ataxia. $553^{-/}$RAG-2 ${ }^{-/}$SCID 
but not $\mathrm{p} 53^{-/}$SCID littermates showed leukemic spread to the leptomeninges and hydrocephalus, without spread to the brain parenchyma. Adoptive transfer studies show that the ability of leukemic pro-B cells to penetrate and proliferate within the leptomeninges in $\mathrm{p} 53^{-/-} \mathrm{RAG}-2^{-/}$SCID mice is tumour cell intrinsic. SKY analysis shows characteristic translocations involving Ig loci in RAG-2 proficient $\mathrm{p} 53^{--} \mathrm{SCID}$ leukemias, and completely different chromosomal aberrations in $\mathrm{p} 53^{-/} \mathrm{RAG}-2^{-/-}$ SCID tumours.

Conclusions: These mice are a novel, spontaneous model of leptomeningeal leukemia. The different spectrum of chromosomal aberrations in $\mathrm{p} 53^{-/}$RAG- $2^{-/}$SCID versus $\mathrm{p} 53^{-/-}$ SCID leukemia suggests that survival in/penetration of the leptomeninges by leukemic cells is not purely stochastic and may be due to specific genetic events.

\section{P-145}

\section{Identification of an arteriovenous fistula in a child: case report and review of the literature}

M. Vassilyadi (Ottawa, Ontario), B.V. Jones, W.S. Ball, Jr. (Cincinnati, Ohio, USA)

Background: Arteriovenous fistulas (AVFs) are occasionally encountered, but not frequently reported. They are distinct from arteriovenous malformations, which outnumber AVFs by over twenty-fold.

Methods: A six-year-old girl sustained a subarachnoid hemorrhage after a mild head injury and was discovered to have a large right mesiotemporal lobe AVF. The etiology of the subarachnoid hemorrhage was not evident until a brain CT with contrast was performed. CT angiography was particularly useful in providing a good look at the lesion in a relatively quick manner. Further studies included brain MRI followed by cerebral angiography.

Results: Two anomalous arteries originating from branches of the right internal cerebral artery were identified supplying fistulas associated with aneurysmal dilatations and drainage into the superficial petrosal sinus. Both fistulas were successfully occluded using endovascular techniques. The child's symptoms of headache and nausea subsequently subsided and a full recovery was made without any neurological deficits.

Conclusion: AVFs are typically present in childhood and may be either congenital or traumatic in origin. The diagnosis is usually delayed and depends on the patient's age, site and size of the fistula, and the effect of any other accompanying vascular lesion. This paper discusses AVFs, their treatment and reviews the literature.

\section{CHILD NEUROLOGY}

\section{P-146}

\section{Botulinum toxin as a treatment for excessive drooling in children with neurological dysfunction}

\author{
J.E. Bothwell, K. Clarke, J.M. Dooley, K.E. Gordon, \\ R. Anderson, E.P. Wood, C.S. Camfield, P.C. Camfield \\ (Halifax, Nova Scotia)
}

Background: Drooling is significant for some neurologically impaired children. Botulinum toxin A (Botox) injection of salivary glands has effectively treated drooling in adults. Botox has not been previously used to treat drooling in children.

Methods: Children identified as having severe daily drooling were enrolled. Pre-injection assessment included measurement of the amount and frequency of drool. Each parotid gland was injected in the preauricular region with 5 units of Botox using a 30 -gauge needle and 1-ml syringe. Post injection assessments have occurred at weeks 2 and 4 .

Results: Five children have been enrolled, aged 4yrs 9mth 14years $2 \mathrm{mth}$. Two patients had an excellent response with little or no drooling. One patient showed a response at week 2, however drooling returned to pre-injection level by week 4 . One patient did not show a response at week 2 but had a mild response at week 4 . One patient did not show any benefit and at week 4 had an increase in drooling. We continue to follow the patients for duration of response. No complications have occurred to date.

Conclusion: Botox appears to be a relatively effective treatment option for some children with significant drooling with no side effects at present.

\section{P-147}

\section{Recurrent oculomotor nerve palsy in a child}

\section{Campbell, P. Jacob, W. Clarke (Ottawa,Ontario)}

Background: Acquired, isolated oculomotor nerve palsies are rare especially in children. The etiologies include infections, neoplasms, trauma, aneurysms and inflammatory demyelinating conditions. These causes generally have a permanent or monophasic clinical nature. Recurrent oculomotor palsies in children have been previously reported in children in the context of ophthalmoplegic migraine, an entity with unclear pathophysiology. Literature has described magnetic resonance imaging (MRI) showing transient enhancement of the cisternal portion of the third nerve in this migraine type. To our knowledge, a report of recurrent oculomotor palsy with MRI enhancement has not been reported with the absence of headache in a child as young as ours.

Methods: The authors report a two-year-old child with recurrent oculomotor nerve palsy.

Results: The child presented at one year of age, 12 days after his 12 months immunization (MMR) with an incomplete isolated left oculomotor nerve palsy. MRI demonstrated hypointense T2 weighted lesion in the cisternal portion of the third nerve that enhanced strongly with gadolinium. The deficits resolved with 
steroids and the MRI normalized. The child has had two reoccurrences of identical symptoms and imaging findings. A short period of vomiting preceded the second event but otherwise no precipitants are evident and headache is absent. Cerebral spinal fluid analysis during the first attack has been normal and infectious agents have not been identified.

Conclusions: An unusual case of recurrent left oculomotor palsy is presented presumably of an inflammatory or migrainous origin.

\section{P-148}

\section{PET scan, MRI and electroencephalographic findings in a child with severe hemiconvulsion-hemiplegia-epilepsy (HHE) syndrome}

C. Deacon, C. Cieuta-Walti, S. Robichaud, D. Bergeron, C. Fisch, F. Bénard, J. Rivest, D. Lécuyer (Sherbrooke, Quebec)

Background: The clinical sequence of hemiconvulsions and hemiplegia followed by chronic epilepsy in children was well described by Gastaut in 1957. The causes of the HHE syndrome are multiple, including vascular, inflammatory, infectious insults or following a prolonged febrile status epilepticus. To our knowledge, PET scan abnormalities in HHE syndrome have never been described.

Results: A previously healthy four-year-old boy was found unconscious, in the morning, in his bed, with twitching of the eyes. At the emergency room, he was deeply comatose and febrile $\left(105^{\circ} \mathrm{F}\right)$. Head CT scan and lumbar puncture (LP) were normal. He was treated with diazepam, phenytoin and phenobarbital, was intubated and transferred to our center. At his arrival, he was deeply unconscious with left hemiplegia without focal movements. The first EEG showed diffuse slowing of the right hemisphere. Initial brain imaging was normal but at 48 hours, MRI showed massive edema involving the entire right hemisphere. Within ten days, the child had two episodes of clinical deterioration with radiological evidence of increased intracranial pressure and right uncal herniation. Cerebral angiography, LP, metabolic and detailed hematologic testing, were normal. A FDG PET study of the brain was done three weeks later and showed increased metabolism involving the right mesotemporal lobe, suggesting an epileptic focus, with hypometabolism of the whole right hemisphere cortex. The child developed right temporal lobe epilepsy one month after the acute episode, with good response to carbamazepine and clobazam but was left with residual hemiplegia.

Conclusion: This is a case of severe idiopathic HHE syndrome likely following a febrile status epilepticus (missed during the night). The FDG PETstudy supports the hypothesis of histotoxic brain damage after prolonged focal epileptic activity.

\section{P-149}

\section{Autosomal dominant fever induced dystonia}

\section{J. Dooley, K. Gordon, W. Stewart (Halifax, Nova Scotia)}

Background: Intermittent dystonia in children is often troublesome: its etiology is elusive and its prognosis may be unknown. Fever induced dystonia, inherited as an autosomal dominant trait with benign prognosis has not been reported.

Methods: Two children with identical histories and symptomatology are presented.

Results: Both children had episodes of dystonia, confined to the lower extremities, beginning at two years of age, and recurring annually initially. Events were preceded by fever for $1 / 4$ to 24 hours, often with vomiting. The dystonia involved extension of the knees with inversion of the feet and dorsiflexion of the great toes. The events were distressing, typically lasted $1 / 4$ to 24 hours, and were not relieved by sleep. Between events, the children were neurologically and developmentally normal. Both children had one parent with a history of similar, but milder symptoms. Multiple investigations, including metabolic studies and imaging were normal. Therapy with phenytoin, carbamazepine, diazepam, haloperidol, levodopa-carbidopa and alcohol were ineffective for one child, while the other has not required any therapy. The events have significantly diminished in frequency for the older of these children, now five and tenyears-old.

Conclusion: Fever induced dystonia is a previously unrecognized but benign movement disorder of childhood.

\section{P-150}

\section{An atypical phacomatosis with white matter calcifications and reversible bilateral stroke-like episodes}

A. Gagnon, S. Verret, J. Mathurin (Quebec City, Quebec)

Background: Sturge-Weber Syndrome (SWS) and KlippelTrenaunay-Weber Syndrome (KTWS) are angiodysplasias that show a spectrum of neurologic involvement with considerable overlap.

Methods: We provide a clinical and radiological description of a six-year-old female patient who presented features similar to SWS and KTWS without all of their classical characteristics.

Results: Clinically, a right periorbital and frontal angioma described at birth is now barely visible. The patient had severe right nerve atrophy, microcephaly, psychomotor retardation, attention-deficit/hyperactivity disorder and no frank limb asymmetry. From 22 months of age, she has presented four episodes of alteration of consciousness, vomiting and occasionally seizures. Two episodes had a transitory left hemiparesis and two lasted several weeks with reversible right and left hemiplegia. The investigations for a metabolic and infectious etiology were all negative. The CTs and MRIs reveal bifrontal and biparietal white matter calcifications, right posterior cerebral aneurysm and significant leptomeningeal enhancement of right hemisphere and left parieto-occipital cortex. The cerebral angiography shows a significant number of irregular vessels with very abnormal capillary phase and no venous abnormalities.

Conclusion: We weren't able to find a similar case in the literature. The subcortical calcifications and the angiographic features are atypical for SWS but described in cases of KTWS. Our patient seems to present a variant of these syndromes. 
P-151

\section{Familial schizencephaly with an $X$-linked inheritance pattern}

\section{P. Humphreys, A. Guberman, A. Hunter, A. Blumenthal, J. Hurteau (Ottawa, Ontario)}

Background: Familial cases of schizencephaly are rare: to date, only six families have been reported, the inheritance patterns appearing to be autosomal recessive (4 families), autosomal dominant (1), or gonadal mosaic (1). One family, with two affected males, was found to have a defect in the homeobox gene EMX2, located at 10q.26.1. We report another kindred with familial schizencephaly in which the pattern is strongly suggestive of X-linked inheritance.

Methods: We performed a comprehensive clinical and MR imaging assessment of two males, related across two generations, with schizencephaly and/or perisylvian cortical dysplasia.

Results: The index case (1) had right spastic hemiparesis from birth, his mother's brother (case 2) a left congenital hemiparesis. Both had intractable epilepsy, moderate mental subnormality, and mild pseudobulbar palsy. MRI in case 1 showed left central open-lip schizencephaly and right perisylvian polymicrogyria. Case 2 showed extensive cortical dysplasia in the right perisylvian area similar to that seen in bilateral perisylvian polymicrogyria, as well as mild cortical dysplasia in the left anterior sylvian area. Another sister of case 2 had a stillborn male child said to have had only "half a brain".

Conclusions: These X-linked cases appear to constitute an overlap between familial schizencephaly and bilateral perisylvian polymicrogyria, the inheritance pattern for which is usually X-linked. A common pathogenetic mechanism for the two syndromes is suggested, with mild impairment of gene expression leading to polymicrogyria, severe impairment to schizencephaly.

\section{P-152}

\section{Does fresh frozen plasma have a place in the treatment of stroke in hemolytic uremic syndrome?}

\section{Jad, H.Z. Darwish, A. Wade, R. Woodman (Calgary,} Alberta)

Background: Hemolytic uremic syndrome (HUS) is defined by microangiopathic hemolytic anemia, thrombocytopenia and acute oliguric renal failure. Central nervous system (CNS) involvement occurs in $30-50 \%$ of patients. Stroke is rare, occurring in $2 \%$. Clinical manifestations are secondary to formation of platelet thrombi in the micro-circulation, endothelial detachment and proliferation without perivascular inflammation. It is postulated thrombosis results from failure of a blood protease system which is overwhelmed after massive release of stored von Willebrand factor from injured endothelium.

Methods: We present two children with HUS who developed hemiplegia. Consecutive clinical examination and neuroimaging tests and electroencephalograms (EEG) were done. Both received hemodialysis, and one fresh frozen plasma (FFP) in addition.
Results: Both patients developed confusion, focal seizures, reduced consciousness and hemiplegia. Evidence of large vessel involvement was seen even though cerebral angiography was not done. The child who received FFP showed progression from an encephalopathic state to suppression in the left central region identifying neuronal drop out on his EEG and his MRI showed ischemia in the left insula, parieto-occipital cortex and bilateral fronto-parietal subcortical white matter. The other child died and post mortem showed occlusion of the first branch of the internal carotid artery.

Conclusion: We speculate that large vessel disease can be a cause for CNS dysfunction in HUS in addition to small vessel microthrombosis. FFPmay play a role in reversing CNS vascular thrombosis in HUS by providing the missing protease inhibitor.

\section{P-153}

\section{Attitudes of medical students towards pediatric neurology}

\section{M.M.S. Jan, N.M. Fida (Jeddah, Saudi Arabia)}

Background: Many medical students have apprehensions and misconceptions regarding children with neurological disorders. Planning strategies to encourage them to pursue a career in pediatric neurology would require assessment of their attitudes and career choices.

Methods: Final year medical students who were completing their pediatric rotation during the 1999-2000 academic year were included prospectively. A structured 30-item questionnaire was designed to examine their attitudes towards pediatric neurology.

Results: 161 students (83 males and 78 females) aged 20-26 years (mean 23, SD 1.2) were included. Most students (81\%) had teaching by a pediatric neurologist and 77-100\% had unfavorable attitudes towards the specialty. However, most students $(92.5 \%)$ felt that neurological disorders are challenging and interesting, more so by those who had teaching by a pediatric neurologist $(\mathrm{p}=0.04)$. Although $79 \%$ considered pediatric neurology as a future career option, only nine $(6 \%)$ selected it as their first choice. Sixty-three (39\%) students were undecided about their first future career choice. Students with grade $\mathrm{C}$ or less were more frequently undecided $(p=0.03)$ and five times more likely to report difficulties in eliciting neurological signs (95\% CI 1.2-21, $\mathrm{p}=0.01)$.

Conclusions: Although most students found neurological disorders challenging and interesting, the majority had unfavorable attitudes towards pediatric neurology. The study findings highlight some aspects that might be targeted to promote student's interest in pediatric neurology and improve their learning experiences.

\section{P-154}

A new autosomal recessive connective tissue-brainmuscle disease?

\section{Jenkins, V. Sui, S. Levin (London, Ontario)}

Background: Marden-Walker syndrome and SchwartzJampel syndrome are rare autosomal recessive disorders which involve connective tissue, brain and muscle. Marden-Walker 
syndrome presents with developmental delay, growth retardation, hypotonia, decreased muscle mass, joint contractures, arachnodactyly, blepharophimosis, micrognathia, immobile facies, and Dandy-Walker cerebral malformation. Schwartz-Jampel differs in including myotonia.

Methods: We report two siblings of consanguineous parents with unusual connective tissue, brain and muscle abnormalities. The connective tissue features include marked skin elasticity, wrinkling of skin in the palms and feet, and skin hyperpigmentation. The brain abnormalities include DandyWalker syndrome, agenesis of the corpus callosum, hypotonia and frontal bossing. The muscle disease includes marked muscle wasting and myopathy. Associated features are syndactyly and distal arthrogryposis. We discuss the clinical features, MRI and EMG findings.

Results: The reported cases in the literature of other connective tissue-brain-muscle diseases are reviewed and compared with our two siblings.

Conclusions: We propose that our two cases, as siblings of a consanguineous union, represent a new autosomal recessive connective tissue-brain-muscle disease.

\section{P-155}

\section{Predictors of long-term physical and cognitive disability for full-term infants born with intracranial hemorrhage}

\section{B.S. Jhawar, A. Ranger (London, Ontario), R.F Del Maestro} (Montreal, Quebec)

Introduction: Previously we identified that thrombocytopenia, forceps instrumentation and indicators of perinatal hypoxia were risk factors for intracranial hemorrhage (ICH). However, the relative importance of these factors on long-term outcome remains unclear.

Methods: This study is based on a retrospective cohort design of newborns infants (GA $\leq 37 \mathrm{wks}$ ) that were diagnosed with ICH. We reviewed all radiological information and classified hemorrhage by compartment, location and severity. Outcomes were measured by telephone interviews and review of medical records on two disability outcomes scales (physical and cognitive). Ordinal logistic regression was used to evaluate risk factors against outcomes.

Results: Sixty-six cases were identified and $84.8 \%$ had complete information on outcome (median follow-up 3.0 years (range $1-10.9$ years)). Multivariate modeling revealed that a platelet count of $<50 \times 10(9) / \mathrm{L}$ was the most important predictor of all outcomes (physical disability: OR=5.9, 95\% CI [1.1-30.4], cognitive disability: $\mathrm{OR}=3.7,95 \% \mathrm{CI}$ [0.8-17.4]). In univariate analyses, forceps instrumentation was significantly associated with improved physical outcome and less severe hemorrhage types. There did not appear to be any relationship between low Apgar scores at one or five minutes or the requirement for resuscitation and radiological hemorrhage severity or disability outcome.

Conclusion: In this study we found that the most important predictor of hemorrhage severity and impaired long-term physical and cognitive disability was ICH associated with thrombocytopenia. Hemorrhage associated with forceps instrumentation tended to be less severe and associated with improved outcome.
P-156

Isolated cerebral vasculitis with excellent response to cyclophosphamide

N. Lowry, J. Yager (Saskatoon, Saskatchewan)

Background: Isolated cerebral vasculitis is a rare entity in childhood and adolescence. Management remains controversial and variable.

Methods: We encountered a 16-year-old who presented acutely with bilateral cerebellar hemorrhages.

Results: Cerebral angiography showed areas of moderate to severe stenosis of small and medium arteries consistent with vasculitis. There was no evidence of systemic vasculitis. His initial treatment was IV pulse methylprednisone and IV cyclophosphamide. He subsequently received IV cyclophosphamide once a month for six months. Repeat cerebral angiography three months after finishing therapy was normal.

Conclusion: Isolated cerebral vasculitis in adolescence may respond very well to IV cyclophosphamide.

\section{P-157}

\section{Monophasic monolesional encephalomyelitis (MMEM)}

N. Lowry, J. Yager, R. Griebel, G. Blevins (Saskatoon, Saskatchewan)

Background: A recent review of 48 cases of childhood CNS inflammatory disease discussed three varieties: acute disseminated encephalomyelitis (ADEM), multiphasic disseminated encephalomyelitis (MDEM) and multiple sclerosis.

Methods: We have encountered two patients with another variant of inflammatory CNS disease who presented with acute ataxia.

Results: MRI scan showed enhancing lesions suggestive of tumour. Both were shown at biopsy to be acute inflammatory demyelinating plagues. Both subsequently responded well to steroid therapy. Both had an excellent recovery without evidence of relapse after nine and $1 \frac{1}{2}$ years respectively.

Conclusion: Monophasic monolesional encephalomyelitis (MMEM) is another variant of CNS inflammatory demyelination which mimics brain tumour and has a good prognosis.

\section{P-158}

\section{Basilar artery thrombosis in children}

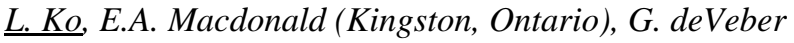
(Toronto, Ontario)

Background: An eight-year-old girl presented with rapidly progressive quadriplegia, ocular bobbing and coma. Basilar artery thrombosis (BAT) was identified on CT, MRA and arteriography. She died despite intra-arterial tissue plasminogen activator and basilar artery stenting. Cases of BAT in the Canadian Pediatric Stroke Registry were reviewed to define the natural history of BAT in children.

Method: All cases of BAT in the Canadian Pediatric Stroke Registry were reviewed with regard to clinical presentation, radiographic investigations and laboratory data. Risk factors, 
outcome and residual neurological deficits were identified where possible.

Results: Of 631 cases of pediatric stroke, 28 had BAT (4\%). Male:female ratio was 3:1. Age range was evenly distributed between one month and 18 years. All cases had neurological deficits at presentation. CT scan identified BAT in 20 patients, MRI in six and angiogram in two. $89 \%$ had one or more risk factors. $79 \%$ received anti-thrombotic therapy. Seven patients recovered fully, 14 had neurological deficits and five expired. No data were available in two patients.

Conclusions: BAT accounts for $4 \%$ of pediatric stroke. Mortality is $18 \%$, which is high for pediatric stroke but compares favorably with the same condition in adults. Risk factors are significantly different from adults. There is no consensus on optimal treatment.

\section{P-159}

\section{A proteomic approach to the inherited lysosomal disease as applied to Chediak Higashi syndrome}

\section{S.H. Pasternak, R.D. Bagshaw, J.W. Callahan,}

\section{D.J. Mahuran (Toronto, Ontario)}

Background: Lysosomes are small intracellular structures which are important in the degradation of proteins, complex lipids, and complex carbohydrates. Relatively little is known about the lysosomal integral membrane proteins or the sorting mechanisms responsible for their localization. To examine these questions, we are studying Chediak-Higashi Syndrome (CHS), an autosomal recessive syndrome in which lysosomal membrane proteins are known to be missorted. In CHS, the defect is due to mutations in LYST, a 429kDa cytosolic protein of unknown function.

Methods: Lysosomes are isolated from both normal and patient fibroblasts by magnetic chromatography after they have endocytosed small iron-dextran particles. Proteins extracted from these lysosomes are then examined by 2D gel electrophoresis.

Results: We estimate that at least 20 (approximately 10\%) of lysosomal membrane proteins are missing from CHS lysosomes.

Conclusions: These experiments demonstrate significant abnormalities in the lysosomal protein constituents. The missorted proteins will be identified using a Micromass Q-ToF Mass Spectometer and comparisons of their cytosolic tail sequences will allow us to derive consensus sequences for protein sorting by LYST. We expect that these studies will provide insights into the basic process of protein sorting and establish a model system for the rapid identification of mutant lysosomal proteins.

\section{P-160}

\section{The clinical spectrum of periventricular nodular heterotopia}

Marie-France Rioux, Yves Robitaille, Jean-François Clement, Anne Lortie, Lionel Carmant (Sherbrooke, Quebec)

Goal: To review the clinical presentation of children with magnetic resonance or pathology proven periventricular nodular heterotopia (PNH).

Background: PNH is a disorder of neuronal migration. It is widely recognized with the advent of MRI as a cause for symptomatic epilepsy. The reported clinical features range from asymptomatic to intractable seizures and mental retardation. Systemic malformations have been reported in certain series, leading to significant morbidity and mortality.

Methods: We retrieved from medical records the charts of all children with a diagnosis of PNH made between 1986-1999. We reviewed their charts to evaluate their clinical signs and symptoms, developmental outcome and epileptic outcome.

Results: We found 28 children with PNH, 21 females and seven males. Nine had unilateral focal, seven bilateral focal and 12 bilateral diffuse PNH. Only two had a positive family history of seizures. Thirteen died in the early perinatal period, with severe systematic malformations including seven with a Chiari type II malformation. Other systemic malformations included cardiovascular, orthopedic, ophthalmologic, genito-urinary, and pulmonary abnormalities. For the 15 children surviving the neonatal period, follow-up ranged from one month- 12 years. Twelve went on to develop epilepsy, which was poorly controlled in four at the time of last visit. EEGs showed multifocal or generalized epileptiform discharges in 11 children. Three had normal recordings and one with a unifocal PNH had focal epileptiform discharges. All 15 children showed a degree of developmental delay. Six were also macrocephalic and five microcephalic.

Conclusion: PNH is part of a disorder of systemic and cerebral malformations. In our population, epilepsy is rarely the principal complaint, as associated malformations lead to significant morbidity and mortality.

\section{P-161}

\section{New insights into the pathogenesis of molybdenum} cofactor deficiency

\author{
M.S. Salman, C. Ackerley, C. Senger, L. Becker(Toronto, \\ Ontario)
}

Background: Molybdenum cofactor deficiency is a rare, progressive neurodegenerative disorder caused by sulphite oxidase enzyme deficiency. The neuropathological findings are consistent with a toxic insult to the brain that causes severe neuronal loss, reactive astrogliosis and spongiosis. The mechanisms responsible for these changes are unknown.

Methods: The case is a male infant with molybdenum cofactor deficiency who died at nine months of age from pneumonia. At autopsy, a complete neuropathological examination was performed including conventional immuno- 
histochemical staining. In addition, brain sections were stained cytochemically for sulphydryl groups. The elemental composition of cortical cells was then analyzed in the scanning electron microscope using backscatter electron imaging and energy dispersive X-ray spectrometry.

Results: Cytochemically, neurons demonstrated excessive cytoplasmic staining with the sulphydryl group when compared to control sections. Energy dispersive X-ray spectrometry analysis of these neurons confirmed the presence of excess sulfur and unexpectedly revealed excess magnesium accumulation.

Conclusions: In molybdenum cofactor deficiency we found abnormal accumulation of sulfur and magnesium in neurons. It is postulated that sulfur-containing compound(s) that are formed as a result of molybdenum cofactor deficiency, cause excitotoxic neuronal injury in the presence of excess magnesium.

\section{P-162}

\section{Neonatal herpes encephalitis - case series of nine cases from Saskatchewan}

\section{Toth, N. Lowry, J. Yager, S. Harder (Saskatoon,} Saskatchewan)

Objective: To describe the clinical and laboratory findings in cases of neonatal herpes simplex virus (HSV) encephalitis.

Background: Neonatal HSV encephalitis is a devastating infection which requires a high degree of clinical suspicion and rapid initiation of antiviral therapy.

Methods: We reviewed nine cases of HSVencephalitis. Cases with consistent clinical presentations were selected for study only when direct evidence of presence of HSV, such as positive cerebrospinal fluid (CSF) viral cultures, positive polymerase chain reaction (PCR) for $\mathrm{HSV}$ from CSF, or positive immunoglobulin $\mathrm{G}$ (IgG) against HSV from blood, was found.

Results: Five male and four female infants were identified. At presentation, seizures occurred in six neonates, lethargy in five neonates, temperature changes in five neonates, and apnea in three neonates. CSF demonstrated an initial monocytosis or lymphocytosis, elevated CSF protein and depressed CSF glucose in all patients. Electroencephalography (EEG) was abnormal in all eight patients with EEG done. Initial computerized tomography was abnormal in five of nine cases. Follow-up over an average of two years demonstrated developmental delay in four patients and upper motor neuron findings in four patients. No patients suffered mortality.

Conclusions: Neonatal HSV encephalitis presents with seizures, lethargy, and dysthermia, and is associated with early CSF monocytosis, abnormal EEG, and leads to significant morbidity after resolution of infection.

\section{P-163}

\section{Hemiplegia following a prolonged latent period after chickenpox}

Elaine Wirrell (Calgary, Alberta), Shauna Kotecha (Ottawa, Ontario)

Case Report: A 2-year 5-month-old boy presented with two bouts of acute-onset left hemiparesis within a five day period.
His past history was significant only for chickenpox five months prior to presentation. A CT showed a hypodensity in the right lenticular nucleus encroaching on the posterior limb of the internal capsule. Coagulation studies, cerebral spinal fluid analysis, echocardiography and carotid ultrasound were normal. MR angiography showed significant narrowing of the proximal right middle cerebral artery and possibly the A1 segment of the anterior communicating artery, in keeping with post-varicella angiopathy.

He was treated with acetosalicylic acid (40 mg/day) and on follow-up one year later, had no recurrent neurological symptoms. A repeat MR angiogram, however, continues to show narrowing of the proximal right middle cerebral artery.

Conclusion: Acute hemiplegia as a result of varicella-induced cerebral angiitis has only recently been described in the literature. This child developed symptoms five months after the onset of primary varicella infection, which is the longest interval between chickenpox exanthem and neurological symptoms thus far described.

\section{P-164}

\section{Parental anxiety and family disruption following a first febrile seizure in childhood}

Elaine Wirrell (Calgary, Alberta), Troy Turner (Edmonton, Alberta)

Objective: To evaluate the degree of parental anxiety and family disruption following a febrile seizure.

Methods: Families of all children presenting to the emergency department with their first febrile seizure were invited to participate in a telephone interview to assess degree of parental anxiety and family dysfunction. Parameters measured included co-sleeping, parental sleep disturbance, fatigue, monitor use, number of night-time checks on child, child-care use, parental outings, fear of missing a seizure, perception of increased vulnerability of child and family stress level. The interview held shortly after presentation compared anxiety/dysfunction in the two weeks immediately preceding to the two weeks following the febrile seizure.

Potential predictors of anxiety/dysfunction including details of presenting seizure, past medical illnesses, family history, socioeconomic status and parental perception of risks of a febrile seizure were queried.

Results: Thirty one of 35 (89\%) families eligible for study participated. Parental anxiety and family dysfunction were significantly greater for nearly all parameters assessed in the two weeks following the seizure. Socioeconomic factors correlated most closely with anxiety/dysfunction prior to the febrile seizure, however were less predictive in the two weeks following the seizure.

Conclusions: Parental anxiety and family dysfunction are the rule following a child's first febrile seizure. Neither higher socioeconomic status nor understanding of the low risk of sequelae with febrile seizures were strongly predictive of improved coping in the two weeks following the seizure. 


\section{INDEX}

\begin{tabular}{|c|c|c|c|c|c|}
\hline Abdoh, A. & P-119 & Bernier, J.P. & P-017 & Campbell, C. & P-147 \\
\hline Abosch, A. & $\mathrm{H}-03$ & Bernier, J.P. & P-040 & Carmant, Lionel & P-160 \\
\hline Abrams, Judith & P-095 & Bernstein, M. & G-03 & Carr, J.A.R. & $\mathrm{C}-02$ \\
\hline Ackerley, C. & P-161 & Bernstein, M. & P-083 & Casha, S. & F-01 \\
\hline Ackroyd, S. & P-127 & Bernstein, M. & P-115 & Casha, Steven & G-01 \\
\hline Addas, B. & P-013 & Berrouschot, Jorg & P-001 & Castellanos, C. & P-049 \\
\hline Akaishi, K. & $\mathrm{K}-02$ & Bertrand, L. & P-002 & Chaalala, C. & P-084 \\
\hline Akiyama, Y. & $\mathrm{K}-02$ & Bilbao, J. & P-081 & Chaalala, C. & P-085 \\
\hline Al-Gahtany, M. & P-081 & Bilbao, J. & P-082 & Chan, K.M. & P-073 \\
\hline Al-Gahtany, M. & P-082 & Bilbao, J.M. & P-090 & Chapman, A. & P-094 \\
\hline Al-Shafai, N. & P-088 & Bilbao, J.M. & P-092 & Chapman, K.M. & P-004 \\
\hline Alemdar, A.Y. & P-068 & Bilbao, Juan & P-096 & Chelyshev, Yu. & P-045 \\
\hline Alexander, P. & P-124 & Bisnaire, D. & P-125 & Chen, Q. & P-095 \\
\hline Allanson, J. & P-043 & Black, S.E. & E-05 & Chevalier, Jean-François & P-005 \\
\hline Andermann, F. & A-02 & Blaser, S. & P-141 & Chevalier, Jean-François & P-086 \\
\hline Andermann, F. & $\mathrm{H}-03$ & Blayney, M. & P-041 & Chiappa, Sharon & K-03 \\
\hline Anderson, R. & P-146 & Blevins, G. & P-157 & Chipman, M. & D-02 \\
\hline Arkia, H. & P-076 & Blount, Jeffrey & $\mathrm{H}-02$ & Chitayat, D. & P-037 \\
\hline Armour, J.A. & P-030 & Blume, W.T. & A-01 & Chitoku, S. & $\mathrm{I}-02$ \\
\hline Armstrong, E.A. & I-05 & Blumenthal, A. & P-151 & Chitoku, S. & P-048 \\
\hline Asselin, J. & $\mathrm{I}-05$ & Boling, W. & $\mathrm{H}-01$ & Chow, V. & P-063 \\
\hline Aziz, Tariq & $\mathrm{J}-03$ & Boling, W. & $\mathrm{H}-03$ & Christian, C. & P-011 \\
\hline Badejo, E. Adeleke & P-104 & Boling, W. & P-054 & Christie, S.D. & D-05 \\
\hline Baeesa, S.S. & P-135 & Boling, W. & P-056 & Christie, S.D. & $\mathrm{K}-01$ \\
\hline Bagshaw, R.D. & P-159 & Bolton, C. & P-003 & Chu, Jason K. & P-006 \\
\hline Baillargeon, P.A. & P-055 & Bolton, C. & P-021 & Chu, Joseph Y. & P-006 \\
\hline Bain, J.R. & P-132 & Bothwell, J.E. & P-039 & Chuang, S. & $\mathrm{I}-03$ \\
\hline Baker, K.A. & $\mathrm{C}-01$ & Bothwell, J.E. & P-146 & Chung, Arthur K. & P-006 \\
\hline Baker, K.A. & P-068 & Bouchard, J-P. & P-044 & Cieuta-Walti, C. & P-148 \\
\hline Ball, Jr., W.S. & P-145 & Bouchard, J.-P. & P-047 & Clarke, B. & P-049 \\
\hline Banwell, B.L. & P-066 & Boulanger, J.M. & P-040 & Clarke, D.B. & D-05 \\
\hline Barber, Philip A. & E-03 & Bourque, P.R. & P-043 & Clarke, D.B. & $\mathrm{J}-04$ \\
\hline Barber, Philip A. & P-001 & Boyle, C. & P-014 & Clarke, D.B. & P-036 \\
\hline Barber, Phillip & E-07 & Brassard, Rolland & P-005 & Clarke, D.B. & P-127 \\
\hline Baruchel, S. & P-141 & Bridge, Peter & C-03 & Clarke, K. & P-146 \\
\hline Bassett, R. & P-029 & Brunet, D. & P-003 & Clarke, W. & P-147 \\
\hline Bastos, A. & P-054 & Brunet, D. & P-044 & Clément, Jean-François & P-160 \\
\hline Basualdo-Hammond, C. & P-052 & Buchan, A.M. & E-04 & Cochrane, D. & P-139 \\
\hline Bazinet, J. & P-085 & Buchan, A.M. & E-05 & Cohen, Gideon & E-02 \\
\hline Becker, L. & P-161 & Buchan, Alastair M. & E-03 & Cole-Haskayne, Andrea & E-03 \\
\hline Becker, L.E. & P-053 & Buchan, Alastair M. & E-07 & Cole-Haskayne, Andrea & E-07 \\
\hline Becker, L.E. & P-141 & Burkholder, L. & B-01 & Corcoran, A. & K-05 \\
\hline Becker, Larry & P-099 & Burkholder, L.M. & P-109 & Cortez, M.A. & B-03 \\
\hline Becker, Laurence & P-098 & Busche, K.D. & $\mathrm{J}-01$ & Cortez, M.A. & B-04 \\
\hline Becker, Laurence & P-143 & Button, Allison & E-03 & Cortez, M.A. & P-050 \\
\hline Beletsky, V. & E-01 & Caines, A. & P-031 & Cossoy, M.B. & P-061 \\
\hline Belkadi, A.M. & P-034 & Callahan, J.W. & P-159 & Couillard, P. & P-023 \\
\hline Bénard, F. & P-148 & Camfield, C.S. & I-01 & Cox, T.C. & $\mathrm{I}-04$ \\
\hline Benstead, T.J. & P-061 & Camfield, C.S. & P-146 & Crevier, L. & P-132 \\
\hline Bercovici, E. & B-04 & Camfield, P.C. & P-039 & Cunnane, S. & P-050 \\
\hline Bergeron, D. & P-148 & Camfield, P.C. & P-146 & Currie, R.W. & P-036 \\
\hline Bernasconi, A. & H-01 & Camfield, P.R. & I-01 & Curtis, R. & P-052 \\
\hline Bernasconi, N. & $\mathrm{H}-01$ & Campbell, C. & P-041 & Cusimano, M. & P-087 \\
\hline
\end{tabular}




\begin{tabular}{|c|c|c|c|c|c|}
\hline Cusimano, M. & P-088 & Dumont, Aaron S. & P-035 & Gloor, P. & A-02 \\
\hline Cusimano, M.D. & D-02 & Dumont, Aaron S. & P-106 & Goguen, J. & P-100 \\
\hline Cusimano, M.D. & P-025 & Dumont, Randall J. & E-02 & Golomb, Meredith & $\mathrm{I}-07$ \\
\hline Cusimano, M.D. & P-089 & Dumont, Randall J. & P-035 & Gordon, K. & P-149 \\
\hline Cusimano, M.D. & P-090 & Duquette, P. & $\mathrm{J}-06$ & Gordon, K.E. & P-039 \\
\hline Cusimano, M.D. & P-100 & Dura, W. & K-03 & Gordon, K.E. & P-146 \\
\hline Cusimano, M.D. & P-101 & Edwards, C. & P-117 & Gorman, P. & $\mathrm{P}-123$ \\
\hline Cusimano, M.D. & P-116 & Ekong, C. & P-130 & Gosselin, F. & P-047 \\
\hline Cusimano, M.D. & P-128 & Elbreki, M. & P-124 & Gosselin, S. & P-017 \\
\hline Cusimano, M.D. & P-129 & Eliasziw, M. & A-01 & Gould, P. & P-084 \\
\hline Cusimano, M. & P-082 & Elson, S. & P-003 & Gould, P. & P-085 \\
\hline Czechowsky, Diana & P-012 & Elson, S. & P-021 & Gould, P.V. & P-044 \\
\hline Danska, Jayne S. & P-144 & Emery, D. & P-009 & Goulet, Benoit & P-107 \\
\hline Darvesh, S. & P-030 & Émond, F. & $\mathrm{P}-047$ & Goulet, Benoit & P-108 \\
\hline Darvesh, S. & P-031 & Emsley, J.G. & P-036 & Graham, J. & P-029 \\
\hline Darwish, H.Z. & P-152 & Etchells, E. & P-115 & Graham, Lisa & $\mathrm{C}-03$ \\
\hline Das, Shyamal & C- 03 & Evoy, F. & P-007 & Griebel, R. & P-110 \\
\hline DaSilva, V.F. & E-06 & Evoy, F. & P-017 & Griebel, R. & P-130 \\
\hline De Almeida, A. & A-02 & Farmer, J.-P. & P-077 & Griebel, R. & P-157 \\
\hline De Almeida, A. & $\mathrm{H}-01$ & Farmer, Jean-Pierre & P-086 & Griebel, R.W. & G-02 \\
\hline de la Torre, J.C. & K-06 & Fay, S. & P-029 & Griebel, R.W. & P-131 \\
\hline de Rosa Jasson, M.F. & P-101 & Fazl, M. & P-109 & Griesdale, D.E. & P-091 \\
\hline de Tilly, L.N. & P-090 & Fehlings, D. & I-06 & Groll, D. & P-003 \\
\hline Deacon, C. & P-148 & Fehlings, M.G. & F-01 & Guberman, A. & P-151 \\
\hline Debruille, J.B. & B-02 & Fehlings, M.G. & K-07 & Gubitz, G. & E-05 \\
\hline Del Duca, T. & P-105 & Fehlings, M.G. & P-076 & Gubitz, G. & P-010 \\
\hline Del Duca, T. & P-133 & Fehlings, Michael G. & G-01 & Gubitz, G. & P-011 \\
\hline Del Duca, T. & P-134 & Fehlings, Michael G. & P-107 & Guha, A. & K-04 \\
\hline Del Maestro, R. F. & K-05 & Fehlings, Michael G. & P-108 & Guha, A. & P-092 \\
\hline Del Maestro, R.F. & D-01 & Fehlings, Michael G. & P-112 & Guha, A. & P-103 \\
\hline Del Maestro, R.F. & P-038 & Fenstermaker, Robert & P-095 & Guidos, Cythia J. & P-144 \\
\hline Del Maestro, R.F. & P-155 & Fewer, D. & P-119 & Hachinski, V. & E-05 \\
\hline Demchuk, Andrew M & E-03 & Fida, N.M. & P-153 & Hader, W.J. & $\mathrm{I}-02$ \\
\hline Demchuk, Andrew M. & P-001 & Findlay, J. Max & D-04 & Hader, Walter & $\mathrm{H}-02$ \\
\hline Demeria, D. & D-07 & Findlay, J. Max & P-009 & Haghighi, S.S. & P-074 \\
\hline Deodhare, S. & P-088 & Findlay, J. Max & P-118 & Hamilton, M.G. & P-120 \\
\hline Derry,P.A. & J-02 & Finkelstein, J.A. & P-109 & Hamilton, M.G. & P-137 \\
\hline Desjardins, A. & P-007 & Finnis, K.W. & P-126 & Hamilton, M.G. & P-138 \\
\hline deVeber, G. & P-158 & Fisch, C. & P-148 & Нао, C. & P-009 \\
\hline Dirks, P. & P-008 & Fisk, J. & P-032 & Harder, S. & P-162 \\
\hline Dirks, P.B. & P-141 & Fisk, J.D. & $\mathrm{J}-01$ & Harms, L. & P-001 \\
\hline Dirks, Peter & P-143 & Fleetwood, I.G. & D-06 & Hattingh, S. & G-02 \\
\hline Doering, L.C. & P-132 & Fleetwood, I.G. & P-119 & He, B.P. & P-046 \\
\hline Doig, G.S. & P-059 & Fox, A.J. & P-111 & Healey, P. & E-06 \\
\hline Donat, J. & P-063 & Frayne, Richard & E-03 & Hentschel, S. & P-091 \\
\hline Dooley, J. & P-149 & Freeman, Carolyn & P-086 & Hentschel, S. & P-139 \\
\hline Dooley, J.M. & P-039 & Fullagar, Tim & P-095 & Herman, Peter & P-102 \\
\hline Dooley, J.M. & P-146 & Furtado, Sarah & C- 03 & Hetzel, Fred & P-095 \\
\hline Doyle-Pettypiece, P. & P-125 & Gagnon, A. & P-150 & Hill, M.D. & E-04 \\
\hline Drake, J.M. & P-136 & Gariépy, J.-L. & P-027 & Hill, M.D. & E-05 \\
\hline Drake, J.M. & P-140 & Gates, L. & P-016 & Hill, Michael D. & E-03 \\
\hline Drake, James & P-099 & Gauthier, Serge & P-033 & Hill, Michael D. & P-012 \\
\hline Dubeau, F. & A-02 & Gillis, M. & P-030 & Hochstein, A. & P-105 \\
\hline Dubeau, F. & $\mathrm{H}-03$ & Girvin, J.P. & A-01 & Hogan, M.J. & E-06 \\
\hline Ducharme, J. & D-03 & Girvin, J.P. & P-051 & Hogg, David & K-03 \\
\hline Dumont, Aaron S. & E-02 & Gladdy, Rebecca A. & P-144 & Hohol, M. & P-066 \\
\hline
\end{tabular}


LE JOURNAL CANADIEN DES SCIENCES NEUROLOGIQUES

\begin{tabular}{|c|c|c|c|c|c|}
\hline Holdsworth, D.W. & P-111 & Jiang, $\mathrm{Na}$ & E-05 & Lee, D.H. & P-111 \\
\hline Holness, R.O. & P-013 & Jiang, Na & P-028 & Lee, Linda & P-113 \\
\hline Honey, C.R. & $\mathrm{C}-02$ & Jobin, P. & P-044 & Lee, $\mathrm{S}$. & K-02 \\
\hline Hopkins, D.A. & P-030 & Jog, M. & P-070 & Lehman, R. & P-056 \\
\hline Horvath, E. & P-100 & Jog, M. & P-071 & Leung, S. & P-058 \\
\hline Horvath, E & P-081 & Jog, M.S. & P-069 & Levin, S. & P-060 \\
\hline Houlden, D. & B-01 & Johnson, A. & P-069 & Levin, S. & P-154 \\
\hline Houlden, D.A. & P-109 & Johnson, S. & P-119 & Levy, R.P. & D-06 \\
\hline Howes, W.J. & P-093 & Jones, B.V. & P-145 & Lewis, $\mathrm{M}$. & P-015 \\
\hline Howes, W.J. & P-094 & Juurlink, B. & P-110 & $\mathrm{Li}, \mathrm{J}$ & D-02 \\
\hline Howse, D. & P-003 & Juurlink, B.H.J. & P-131 & Lilge, Lothar & P-095 \\
\hline $\mathrm{Hu}$, William & E-03 & Kaibara, T. & P-120 & Lingwood, C.A. & P-101 \\
\hline Hudon, Mark E. & E-03 & Kaibara, T. & P-138 & Liu, Ling & K-03 \\
\hline Huq, N.S. & P-132 & Kalapos, P. & E-06 & Liu, X. & P-058 \\
\hline Hui, C.C. & $\mathrm{K}-03$ & Kalnins, I. & P-128 & Liu, Y. & P-052 \\
\hline Humphreys, P. & $\mathrm{P}-151$ & Kamencic, H. & P-110 & Lobay, G.L.W. & P-073 \\
\hline Humphreys, Robin & P-143 & Kamencic, H. & P-130 & Lortie, Anne & P-160 \\
\hline Hunjan, A. & P-048 & Kan, $P$. & P-087 & Louissi, S. & P-076 \\
\hline Hunter, A. & P-151 & Karaskova, Jana & P-144 & Louissi, S. & P-078 \\
\hline Hurlbert, R. John & P-106 & Kasner, Scott E. & P-001 & Lownie, S.P. & $\mathrm{P}-111$ \\
\hline Hurteau, J. & P-151 & Kassell, Neal F. & E-02 & Lowry, N. & P- -080 \\
\hline Hussein, M.S. & G-02 & Kassell, Neil F. & P-035 & Lowry, N. & P-156 \\
\hline Hutchinson, Z. & P-060 & Katzberg, Hans & $\mathrm{J}-03$ & Lowry, N. & P-157 \\
\hline Hwang, S.W. & P-114 & Keith, R. & P-131 & Lowry, N. & P-162 \\
\hline Hyndman, M. Eric & $\mathrm{E}-02$ & Kelly, M. & P-110 & Lussier, Isabelle & P-033 \\
\hline Hyson, H.C. & P-069 & Kelly, M. & P-130 & Lyon, A. & P-110 \\
\hline Ibrahim, H.H. & P-135 & Kerr, E. & I-03 & MacAuley, A. & P-039 \\
\hline Ingles, J. & P-032 & Kestle, J. & P-139 & Macdonald, E.A. & P-158 \\
\hline Intrilligator, James & $\mathrm{J}-05$ & Khan, S.H. & G-02 & Mackay, M.T. & P-053 \\
\hline Isaac, D. & D-02 & Kim, Ji Soo & $\mathrm{J}-05$ & Mackey, A. & P-027 \\
\hline Isaac, D. & P-116 & Kim, Ji Soo & P-065 & MacSween, J. & P-039 \\
\hline Isaac, D. & P-128 & Klein, D. & P-054 & Maharaj, M. & $\mathrm{P}-123$ \\
\hline Jackson, A. & P-003 & Knazzen, M. & P-067 & Mahuran, D.J. & P-159 \\
\hline Jacob, P. & P-041 & Ko, L. & P-158 & Mainprize, T.G. & $\mathrm{K}-02$ \\
\hline Jacob, P. & P-147 & Kontos, C. & K-04 & Mainprize, T.G. & P-037 \\
\hline Jacques, F.H. & P-042 & Korngut, L.M. & P-051 & Mainprize, T.G. & P-092 \\
\hline Jacques, F.H. & P-075 & Kotecha, Shauna & P-163 & Mainprize, T.G. & P-141 \\
\hline Jacques, L. & P-105 & Kovacs, $\mathrm{K}$. & P-081 & Mainprize, Todd & P-098 \\
\hline Jacques, L. & P-133 & Kovacs, K. & P-082 & Mainprize, Todd G. & K-03 \\
\hline Jacques, L. & P-134 & Kovacs, K. & P-100 & Mainprize, Todd G. & P-099 \\
\hline Jacques, S. & $\mathrm{J}-06$ & Krassioukov, A. & P-078 & Mainprize, Todd G. & P-143 \\
\hline Jad, L. & $\mathrm{P}-152$ & Krassioukov, A.V. & P-076 & Maleki, B. & B-02 \\
\hline Jan, M.M.S. & $\mathrm{I}-04$ & Krieger, Derk & P-001 & Malik, S. & D-07 \\
\hline Jan, M.M.S. & P-135 & Krueger-Naug, A.M.R. & P-036 & Maloney, W. & P-013 \\
\hline Jan, M.M.S. & P-153 & Kulkarni, A.V. & P-140 & Mansfield, E. & D-02 \\
\hline Jarjoura, S. & P-018 & Kumar, K. & D-07 & Mansfield, E. & P-128 \\
\hline Jarrett, J. & E-05 & Kumar, R. & P-031 & Marcellus, M. & D-06 \\
\hline Jarrett, J. & $\mathrm{P}-011$ & Labrecque, R. & $\mathrm{J}-06$ & Marchie, A. & P-089 \\
\hline Jenkins, M. & P-070 & LaCaille, D. & P-067 & Marks, M.P. & D-06 \\
\hline Jenkins, $\mathrm{M}$. & P-071 & Lad, T. & P-014 & Marotta, T. & P-019 \\
\hline Jenkins, $\mathrm{M}$. & P-154 & Lamarche, J.B. & P-018 & Marotta, T. & P-020 \\
\hline Jetté, N. & P-043 & Lamarche, J.B. & P-040 & Marotta, Thomas & P-082 \\
\hline Jhawar, B.S. & D-01 & Lamberti-Pasculli, M. & $\mathrm{P}-140$ & Marshall, K.H. & P-114 \\
\hline Jhawar, B.S. & $\mathrm{P}-121$ & Langburt, William & $\mathrm{H}-02$ & Martin, E. & $\mathrm{P}-031$ \\
\hline Jhawar, B.S. & $\mathrm{P}-122$ & Lécuyer, D. & P-148 & Martzke, J.S. & $\mathrm{C}-02$ \\
\hline Jhawar, B.S. & P-155 & Lee, D. & P-070 & Massicotte, E. & P-115 \\
\hline
\end{tabular}




\begin{tabular}{|c|c|c|c|c|c|}
\hline Massicotte, P. & P-136 & Norris, John & P-028 & Richard, S. & P-134 \\
\hline Mathurin, J. & $\mathrm{P}-150$ & O'Gorman, Augustin M. & P-086 & Riopelle, R. & P-003 \\
\hline Matshes, E. & $\mathrm{P}-110$ & Oakley, H. & D-03 & Riopelle, R.J. & P-021 \\
\hline McAlister, V.C. & P-068 & Obayan, A. & P-131 & Rioux, Marie-France & P-160 \\
\hline McGill, M. & K-02 & Ochi, A. & $\mathrm{I}-02$ & Rivest, Josee & $\mathrm{J}-05$ \\
\hline McGlade, J. & K-02 & Oger, J. & P-004 & Rivest, J. & P-148 \\
\hline McLachlan, R.S. & $\mathrm{J}-02$ & Oger, Joel & $\mathrm{J}-03$ & Roberts, S. & P-031 \\
\hline McLeod, K. & P-142 & Ogunyemi, A. & P-062 & Robichaud, S. & P-148 \\
\hline McNeely,P.D. & P-016 & Olivier, A. & $\mathrm{H}-01$ & Robitaille, Yves & P-160 \\
\hline McNeely,P.D. & P-093 & Olivier, A. & P-054 & Rocker, G. & D-05 \\
\hline McNeill, John H. & P-035 & Olivier, André & A-02 & Rockwood, K. & P-029 \\
\hline McTaggart, M. & E-05 & Olivier, André & $\mathrm{H}-03$ & Rockwood, K. & P-032 \\
\hline Meffe, F. & P-025 & Olivier, André & P-055 & Rodriguez, C. & P-116 \\
\hline Mehta, V. & P-016 & Olivier, André & P-056 & Rodriguez, C. & P-129 \\
\hline Mehta, V. & P-093 & Otsubo, H. & $\mathrm{I}-02$ & Roncari, L. & K-04 \\
\hline Mehta, V. & P-094 & Otsubo, $\mathrm{H}$. & $\mathrm{I}-03$ & Roncari, L. & P-092 \\
\hline Mélançon, Denis & P-005 & Otsubo, $\mathrm{H}$. & P-048 & Roncari, L. & P-103 \\
\hline Melanson, M. & P-003 & Otsubo, $\mathrm{H}$. & P-053 & Rowed, D. & B-01 \\
\hline Mendez, I. & $\mathrm{C}-01$ & Otsubo, Hiroshi & $\mathrm{H}-02$ & Rowed, D. & P-097 \\
\hline Mendez, I. & K-01 & Panu, N. & P-097 & Rowed, D.W. & P-109 \\
\hline Mendez, I. & $\mathrm{P}-034$ & Park, J.S. & K-06 & Rubini, Frank D. & P-022 \\
\hline Mendez, I. & P-068 & Park, Paul C. & P-098 & Rutka, J. & $\mathrm{I}-03$ \\
\hline Mendez, I. & P-123 & Parrent, A. & P-071 & Rutka, J. & P-053 \\
\hline Mendonca, D. & P-071 & Pasternak, S.H. & P-159 & Rutka, J.T. & I-02 \\
\hline Metz, L.M. & $\mathrm{J}-01$ & Paterson, P. & P-110 & Rutka, J.T. & $\mathrm{K}-02$ \\
\hline Meyer, S.H. & P-074 & Patterson, J. & P-129 & Rutka, J.T. & P-037 \\
\hline Mezei, M. & P-067 & Persad, V. & B-03 & Rutka, J.T. & P-048 \\
\hline Midha, R. & P-109 & Peters, T.M. & P-126 & Rutka, J.T. & P-101 \\
\hline Milner, B. & $\mathrm{P}-054$ & Phan, Nicolas & $\mathrm{K}-07$ & Rutka, James T. & $\mathrm{H}-02$ \\
\hline Milot, G. & P-085 & Phillips, S. & E-05 & Rutka, James T. & K-03 \\
\hline Mitchell, Ross & E-07 & Phillips, S. & P-011 & Rutka, James T. & P-098 \\
\hline Mittal, S. & P-077 & Picard, C. & P-085 & Rutka, James T. & P-099 \\
\hline Montanera, W. & P-024 & Porter, P. & P-024 & Rutka, James T. & P-143 \\
\hline Montes, Jose L. & P-086 & Poulin, C. & P-077 & Rutka, James T. & P-144 \\
\hline Morris, M. & $\mathrm{J}-02$ & Poulin, N. & $\mathrm{H}-03$ & Ryan, M.A. & P-050 \\
\hline Morrish, William & E-03 & Poulin, N. & P-055 & Ryckborst, Karla & E-03 \\
\hline Morrison, D. & D-05 & Powell-Vinden, B. & P-015 & Sadi, D. & $\mathrm{C}-01$ \\
\hline Morshead, Cindi M. & P-113 & Purdy, M. & $\mathrm{C}-01$ & Sadi, D. & P-068 \\
\hline Moscarello, M.A. & P-066 & Quesney, F. & $\mathrm{A}-02$ & Sadler, R.M. & $\mathrm{J}-04$ \\
\hline Muhajarine, N. & $\mathrm{P}-130$ & Quirion, A. & P-018 & Sadler, R.M. & P-057 \\
\hline Mukhida, K. & C-01 & Rabin, D. & P-140 & Sadler, R.M. & P-061 \\
\hline Muller, Paul & G-04 & Raffel, Corey & $\mathrm{K}-03$ & Sahjpaul, R. & P-125 \\
\hline Muller, Paul & P-095 & Raginov, I. & P-045 & Sahjpaul, R.L. & P-111 \\
\hline Muller, Paul & P-096 & Rahey, S. & $\mathrm{J}-04$ & Salame, J.A. & P-023 \\
\hline Muller, Paul & P-102 & Rahey, S. & P-057 & Salehi Fateme & P-089 \\
\hline Murphy, D. & P-062 & Rajput, A. & P-063 & Salhia, B. & P-101 \\
\hline Myers, T.L. & P-036 & Rajput, A. & P-072 & Salman, M.S. & P-161 \\
\hline Myles, L.M. & P-136 & Ramanathan, U. & D-02 & Saqqur, Maher & P-064 \\
\hline Myles, S.T. & P-120 & Ranger, A. & D-01 & Sarjeant, R. & P-076 \\
\hline Myles, S.T. & P-138 & Ranger, A. & P-155 & Sarjeant, R. & P-078 \\
\hline Nabil Hussain, N. & P-070 & Reddy, K. & P-124 & Satkunam, L. & P-073 \\
\hline Nadeau, A. & P-017 & Redekop, G. & P-019 & Satomi, J. & P-024 \\
\hline Neville, B.G.R. & I-04 & Redekop, G. & P-020 & Savard, G. & A-02 \\
\hline Newcommon, N. & E-05 & Resch, L. & P-093 & Scherer, Steve & K-03 \\
\hline Newcommon, Nancy J. & E-03 & Richard, S. & P-133 & Schwartz, Gwen & P-112 \\
\hline Norris, J.W. & E-01 & & & Schwartz, Gwen & P-113 \\
\hline
\end{tabular}


LE JOURNAL CANADIEN DES SCIENCES NEUROLOGIQUES

Schwartz, M.L.

Schwarz, B.A.

Scott, R.C.

Selker, Robert

Senger, C.

Serbanescu, I.

Shagidullin, T.

Sharman, A.

Sharpe, James A.

Sharpe, James A.

Sharpe, $\mathrm{M}$.

Sherlock, R.

Shuaib, A.

Sibbald, T.

Silver, $\mathrm{K}$.

Sin, N.C.

Sinden, M.

Singer, W.

Singh, S.K.

Singhal, A.

Smith, M.L.

Smyth, H.

Smyth, H.S.

Smyth, H.S.

Snead, O. Carter

Snead, O.C.

Snead, O.C.

Snead III, C.

Snead III, O.C.

Snead III, O.C.

Snead III, O.C.

Snead III, O.C.

Sonntag, Volker K.H.

Sopher, Laurel

Soualmi, L.

Squire, Jeremy

Squire, Jeremy A.

Sschmutzhard, E

Starreveld, Y.P.

Steinberg, G.K.

Steinbok, P.

Steinbok, P.

Stephens, D.

Stewart, Duncan J.

Stewart, W.

Stoessl, A.J.

Stone, Scellig S.

Strong, M.J.

Stys, P.

Suchowersky, Oksana

Sui, V.

Sutherland, G.R.

Sutherland, G.R.

Sutherland, Garnette R.

Sutherland, Garnette R.

\begin{tabular}{|c|c|}
\hline P-109 & Sweeney,P.J. \\
\hline E-05 & Tallon, J.M. \\
\hline $\mathrm{I}-04$ & Tampieri, Donatella \\
\hline P-095 & Tator, C. \\
\hline P-161 & Tator, C.H. \\
\hline $\mathrm{P}-050$ & Tator, Charles H. \\
\hline P-045 & Taylor, M.D. \\
\hline D-02 & Taylor, M.D. \\
\hline $\mathrm{J}-05$ & Taylor, M.D. \\
\hline P-065 & Taylor, M.D. \\
\hline P-058 & Taylor, Michael D. \\
\hline P-041 & Taylor, Michael D. \\
\hline E-01 & Taylor, Michael D. \\
\hline P-116 & Taylor, Michael D. \\
\hline P-077 & Taylor, Michael D. \\
\hline $\mathrm{I}-03$ & terBrugge, $\mathrm{K}$. \\
\hline C- 02 & Theodore, Nicholas \\
\hline P-082 & Thomas, $\mathrm{T}$. \\
\hline P-025 & Thornhill, J. \\
\hline G-03 & Tomanek, Anna \\
\hline $\mathrm{I}-03$ & Toth, C. \\
\hline P-081 & Toth, C. \\
\hline P-089 & Toth, C. \\
\hline $\mathrm{P}-100$ & Toth, C. \\
\hline $\mathrm{H}-02$ & Tsai, E.C. \\
\hline $\mathrm{I}-03$ & Tucker, William \\
\hline P-053 & Turmel, A. \\
\hline $\mathrm{I}-02$ & Turmel, A. \\
\hline B- 03 & Turner, Troy \\
\hline B-04 & Tyagi, A. \\
\hline P-048 & Tymianski, M. \\
\hline P-050 & Tymianski, M. \\
\hline P-106 & Upton, A. \\
\hline E-03 & Ursell, M. \\
\hline P-056 & Vajsar, J. \\
\hline P-098 & van Bendegem, R. \\
\hline P-144 & van der Kooy, Derek \\
\hline P-001 & van Furth, W.R. \\
\hline P-126 & van Furth, W.R. \\
\hline D-06 & Vanderaa, D. \\
\hline P-139 & Vandorpe, R. \\
\hline P-142 & Varma, A. \\
\hline $\mathrm{I}-06$ & Varma, A. \\
\hline P-035 & Varma, Abhay \\
\hline P-149 & Vassilyadi, M. \\
\hline P-067 & Verma, Subodh \\
\hline P-113 & Verma, Subodh \\
\hline P-046 & Verreault, S. \\
\hline E-05 & Verreault, S. \\
\hline C-03 & Verret, $\mathrm{S}$. \\
\hline P-154 & Verro, P. \\
\hline $\mathrm{P}-120$ & Veugelers, P. \\
\hline P-138 & Voll, C. \\
\hline E-02 & Voll, C. \\
\hline P-035 & von Ritschl, R. \\
\hline
\end{tabular}

P-079

P-127

P-005

P-078

P-114

P-113

K-02

P-037

P-121

P-141

K-03

P-098

P-099

P-143

P-144

P-024

P-106

J-06

I- 05

E-07

J-07

P-026

P-080

P-162

P-114

G-04

P-084

P-085

P-164

I-02

P-008

P-024

P-049

P-066

I-06

P-114

P-113

P-100

P-101

I-02

P-013

P-090

P-100

G-04

P-145

E-02

P-035

P-027

P-047

P-150

P-001

I-01

J-07

P-026

P-013
VonRitschl, R.

Vorobeychik, G.

P-016

Vujovic Zotovic, Nada

P-067

Wade, A.

Wallace, M.C.

Walling, S.

Walling, S.

Walling, S.

Walsh, R.

Weaver, D.

Weill, A.

Weill, A.

Weiss, S.

Weiss, S.

Weiss, S.K.

Wells, J.

Wennberg, R.A.

Wentzel, C.

Wentzel, C.

Werbowetski, T.

Wheatley, Matt

Wiebe, S.

Wiebe, S.

Wilbourn, A.J.

Wilkins, G.

Williams, Christine J.

Williams, R.

Williams, S.

Willinsky, R.

Wilson, Brian

Wilson, Brian

Wirrell, Elaine

Wirrell, Elaine

Wood, E.P.

Woodman, R.

Woolfenden, A.

Wyldman, S.

Yager, J.

Yager, J.

Yager, J.

Yager, J.Y.

Yang, Victor

Yang, Victor

Yhap, P.

Young, B.

Young, B.

Young, B.

Zadeh, G.

Zadeh, G.

Zhang, Jinjin

Zhang, X.
P-028

P-152

P-024

D-03

P-094

P-123

P-031

$\mathrm{P}-003$

P-019

P-020

I-03

P-048

P-053

P-117

P-037

P-029

P-032

P-038

H-01

A-01

P-069

P-079

P-091

P-144

P-049

P-052

P-024

P-095

P-102

P-163

P-164

P-146

P-152

E-01

P-073

P-156

P-157

P-162

I-05

P-095

P-102

P-094

P-058

P-059

P-060

K-04

P-103

P-001

K-03 


\section{CANADIAN ASSOCIATION OF NEUROSCIENCE NURSES JUNE 13- 15, 2001 HALIFAX, NOVA SCOTIA}

\section{Posters}

The Development and Integration of a Neuroscience Program at Children's and Women's Health Centre of British Columbia

Suzanne Payne - BSN, RN, General Staff Nurse, Brenda Won-BSN, RN, General Staff Nurse

\section{Neuroscience Step Down Unit Experience}

Jacqueline Willems - Clinical Educator, Neurosciences, Christine Thomas - Care Leader, Herta Yu - Staff Nurse, Tuulikki Ilvonen - Staff Nurse

\section{Research Evidence for Neuroscience Nursing} Marlene Reimer-RN, PhD, CNN(c), Associate Professor and Associate Dean, Research and Graduate Programs; Associate in Nursing for Neuroscience

\section{Neuroleptic Malignant Syndrome: A Review}

Wendy Blackburn, RN, BScN, M(Ed), CNN(c), CRRN, Community Liaison Co-ordinator, Lee-Anne LeBlanc, RN, CRRN

\section{Outcomes Following Treatment With Intravenous} Tissue-Plasminogen Activator (Iv Tpa) In Halifax

J. Jarrett - Stroke Research Nurse, S. Phillips - Neurologist, G. Gubitz-Neurologist

\section{You're Getting on My Cranial Nerves!}

Elizabeth Gray, R.N., CNN(C), Neurosciences and Neuro ICU Nurse, Cindy Hartley, R.N., CNN(C), Neurosciences and Neuro ICU Nurse, Donna Brett, R.N., Neurosciences and Neuro ICU Nurse

The Challenges of Introducing SPECT at the Montreal Neurological Hospital

Naomi Akazawa - BScN, CNN(C), Assistant Head Nurse, Isabelle Martineau - BScN, CNN(C), Assistant Head Nurse
Management of Spinal Headache: A Review of the Literature

Sandra Robinson - RN, MN, Acute Care Nurse Practitioner - Spinal Program

Thalamic Stimulation for Intractable Epilepsy

Sonia Sarkissian - RN, MScN, CNN( c) Acute Care Nurse

Practitioner, Richard Wennberg - MD, Andres Lozano-MD

Dysphagia and the prediction of aspiration in Myasthenia Gravis

Wilma J. Koopman - RN, MScN, Nurse

Practitioner/Clinical Nurse Specialist

Co Authors: Samuel Wiebe MD, FRCPC, Angela Colton-

Hudson M Cl. Sc.,Reg. CASLPO, Taslim Moosa M Cl. Sc., Reg. CASLPO, Dean Smith B. MSc., MD, FRCPC, David Bach MD, FRCPC, and Michael Nicolle MD, FRCPC, D. Phil.

Pain Management in a Neuroscience Population: How Well Are We Doing?

Carole White - Clinical Nurse Specialist, Sylvia de MeloStaff Nurse, Patricia Kerr - Head Nurse, Yasmin KhaliliClinical Nurse Specialist, Diane Lowden - Clinical Nurse Specialist, Noelene McGuire - Staff Nurse, Laurel Lowry Assistant Head Nurse

Implementation of a Tracheostomy Weaning Protocol Diane Duff - Professor, Centennial College, Linda YetmanCNS/NP, Neurosurgery

A Descriptive Study of Head Injury Discharges in Newfoundland, 1985-1999

Lisa Clake - Division Manager

Co authors: George Fox, Falah Maroun, Sharon Buehler

Improving End of Life Care in the ICU

Maggie Winstanley, Dawnelda Murray 
Hypotension, Hypoxia and Increased Intracranial Pressure: A Deadly Combination in the Head Injured Patient

Leslie Penny- RN, BScN, Clinical Instructor, Bambi Collins $-R N, B S c N$, Staff Nurse

\section{Deep Brain Stimulation}

Judy Kirby

\section{Collaborative Education in Pediatric Neuroscience} Nursing

Cathy Massey - RN, BScN, Clinical Nurse Specialist, Yvonne Moritz - RN, BScN, Med, Suzanne Tinning - RN, $B S c N$, Clinical Instructor for Neurosciences, Theresa Erb$R N, B S c N$, Clinical Nurse Leader, Neuroscience

\section{Pediatric Neuroscience Preceptor Pathways}

Arlene McKinnon - RN, BScN, Neurology Clinic Nurse, Suzanne Tinning - RN, BScN, Clinical Instructor for

Neurosciences
The Ins and Outs of Femoral Sheaths: Nursing Assessment and Interventions

Sue Duncliffe - Neuroscience Nurse, Mary Trainor Neuroscience Nurse

\section{Neuroscience and Addictions}

Gareth J. Daniels - RN, Drug and Alcohol Program Coordinator

\section{Shaken Baby Syndrome}

Wanda Heading on behalf of the Nova Scotia Committee for the Awareness, Prevention and Management of Shaken Baby Syndrome 


\section{"Topamax}

topiramate

25,100 and $200 \mathrm{mg}$ Tablets and

15 and $25 \mathrm{mg}$ Sprinkle Capsules

Antiepileptic

\section{INDICATONS AND CLINICAL USE}

TOPAMAX (trpiriomute) is indicoted as adiunctive therapy for the monogement of potients (adults ond chilhten two years and older) with epilepsy who ore not sotistactorily controllad with convertional theropy. There is limited information on the use of topicancte in monotheropy of this time. COMIRAINDICATIOHS

TOPAMAX (topiramote) is contraindicated in potients with a history of hypersensifivity to any componennts of this product. WARHHOS

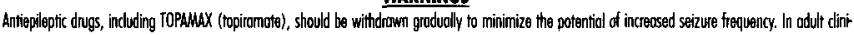
col trials, dosages were deccersed by $100 \mathrm{mg} /$ day of weekly intervals.

Control Nervous System Elfocts

Adverse events most often associnted with the use of TOPAMAx were centrol nervous systemr-elateded. In adults, the most significant of these can be classfied into two gerneral categgories: i) psychomator slowing: difficully with concentration and speech or languoge problems, in particular, wordfinding difficuhies and ii) somnolences or fatigue.

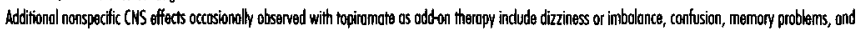

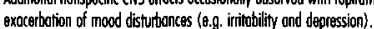

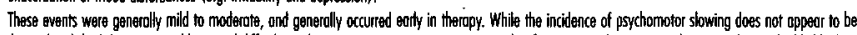
dose related, both languoge problems and difficully with concentration or attention increased in frequency with increessing dosang in the six doublebblind tir ols, suggasting that thase eventis ore dose reloteded. (SOA ADVERSE REACTIONS.)

\section{Effocts Reloted to Cotonk Anhydrose Inthiltion}

$$
\text { PRECAUTIONS }
$$

Kidney Stones A ton of $32 / 1,715$ (1.5\%) of patients axposed to TOPAMAX (topiramote) during its development reported the occurrence of kidney stones,

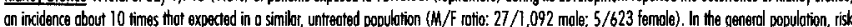

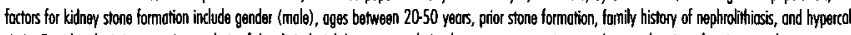

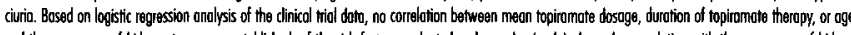
and the occurrence of kidney stoness wos estoblishad; of the risk factors evoluated, only gender (molla) showed a correlotion with the occurrence of kidney stoness. In the padiaticic patients studiad, there were no kidney stones observed.

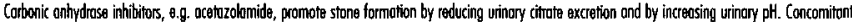

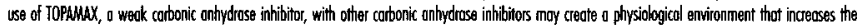
risk of kidney stone formation, and should thereforo be covoided.

Potients, especially those with o predisposition to nephriolithiusis, may have un increassed isk of renal stone formation. Increased fluid intoke increoses the uninory output, lowering the concentetion of substonces involved in stone formation. Therefore, odequate hydration is recommended to reduce this risk. None of the risk factors for naphrolitiniasis con relinbly predict stone formation during TOPAMAX neatment.

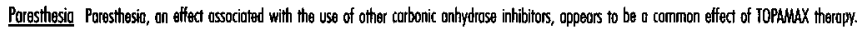
These events were usually intsmittent and mild, and not necessarily reloted to the dosage of topiromate.

Nerifilond Supplementution

A dietary supplement or increasad food intrke may be considered if the patient is lasing weingth while on this medication.

\section{Woight Loss in Podlotrites}

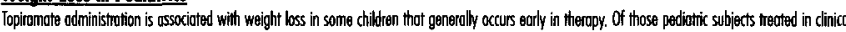
triols for at laost o year who experienced weight loss, $96 \%$ showed o resumption of weight gain within the period tastied. In 2-4 year olds, the mean change in weight from baseline of 12 months ( $n=25$ ) wos $+0.7 \mathrm{~kg}$ (rongeg -1.1 to 3.2 ); at 24 months ( $\mathrm{n}=14$ ), the mean chonge wos +2.2 (ronge -1.1 to 6.1 ).

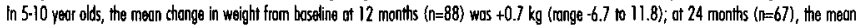
chonge was +3.3 (ronge -8.6 to 20.0). Weight decreocses, usually ossociated with anorexia or appesite chonges, were reported as adverse events for $9 \%$

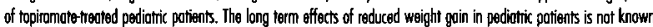

\section{Adjustuent of Dose in Ronel Foilure}

The maior coute of elimination of unchenged topiramate and its metobolites is via the kidnay. Renal alimination is dependent on renal function and is inde pendent of age. Patients with impaired renal function $\left(a_{a}<70 \mathrm{~mL} / \mathrm{min} / 1.73 \mathrm{~m}^{2}\right)$ or with end stoge rencl diseose receiving hemodialysis treatments mor

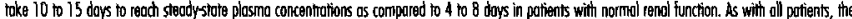

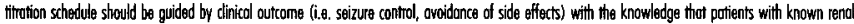
impoiment moy require a longer fime to reach steadystote ot ench dose. (Seg DOSAGE AND ADMINISTRATION.)

Decreosed Hepotic Function

In hepatically impaired patients, topiriomate should be administered with caution os the claoronce of ropiramate was decreased compared with normal sub jects.

Informotion for Potients

Adequate Hydrotion Potients, esspeciolly those with prectisposing foctors, should be instructed to mointoin on adequate fivid intake in order to minimize the risk of renal stane formetion.

Effects on Ability to Dive and Use Moctines

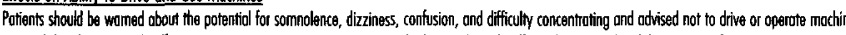
eny unit they howe gainad sufficient experience on topiramotes to gauge whether it odversely offects thair mentral ond/or motor performonce.

Drug Interestions

Antiegileotic Druas

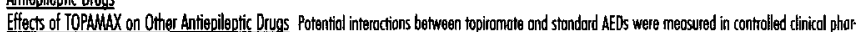

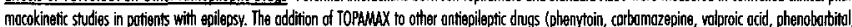
primidone) has no effect on their steady-sotra plosmo concentrotions, except in the occasional patient, where the addition of TOPaMaX to phenytoin may ressult in an increass of plosma concentrations of phenytoin.

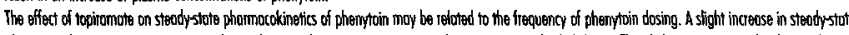
phenytoin plosmo concentrotions wos observed, primanily in patients receiving phenytoin in two divided doses. The sight increose may be due to the sat uroble nature of phentroin pherrmocokinetics ond inhibition of phenytoin metabolism (CYP2C).

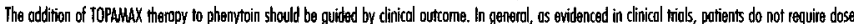

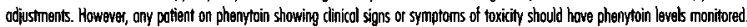

Effects of Other Antiepileptic Drugs on TOPAMAX Phenytoin and contamazepine decreose the plasmo concentration of TOPAMAX. The oddition or with

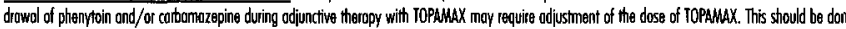
by titrating to elinical effect. The addition or withdrowal of valproic acid does not produce clinically significant changes in plasmo concentrofions of TOPAMaX, ond thereforer, doas not warront dosogg odijustment of TOPAMAX.

The effact of thase interoctions on plosmb concentiotions ore summanized in lable 1 :

Toble 1

Drug Intesactions with TOPAMAX Theropy

\begin{tabular}{|c|c|c|}
\hline $\begin{array}{l}\text { AED } \\
\text { Cood ministered }\end{array}$ & $\begin{array}{c}\text { AED } \\
\text { Concentration }\end{array}$ & $\begin{array}{c}\text { TOPAMAX } \\
\text { Concentrofion }\end{array}$ \\
\hline Phenytoin & $\leftrightarrow^{\star \star}$ & $\downarrow 59 \%$ \\
\hline Contamozepine (CB2) & $\leftrightarrow$ & $\downarrow 40 \%$ \\
\hline CBz epoxide* & $\leftrightarrow$ & NS \\
\hline Valproic acid & $\downarrow 11 \%$ & $\downarrow 14 \%$ \\
\hline Phanobarbitral & $\leftrightarrow$ & NS \\
\hline Primidon日 & $\leftrightarrow$ & NS \\
\hline
\end{tabular}

Is not administerad but is an active metrobolite of carbomazepine

$\leftrightarrow \quad$ No effect on plasmo concentration (< $15 \%$ change)

$\begin{array}{ll}\leftrightarrow & \text { No effect on plasmo concentration (< } 15 \% \text { change) } \\ \star * & \text { Plosma concentrotions increased } 25 \% \text { in some patients, generally those on a bi.d. dosing regimen of phenytoin } \\ \downarrow & \text { Plasmo concentrotions decreose in individual patients }\end{array}$

NS Not studied

AED Antiapileppic drug
Other Drug Interoctions

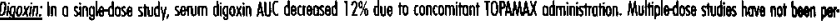

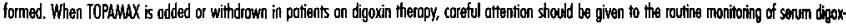

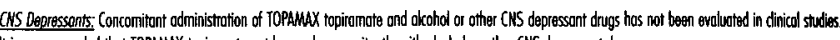
It is recommended that TOPAMAX tapinanote not be used concomituntly with alkohol or other CNS depressont drugs.

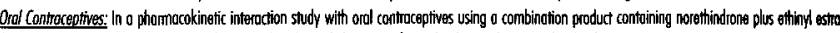

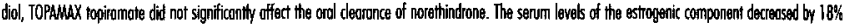

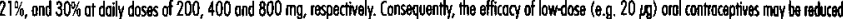

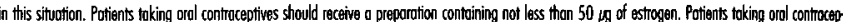
fives should be asked to report any change in their bleeding potterms.

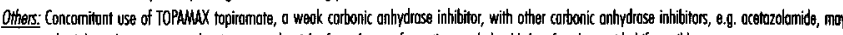
create a physiological environment that incrososes the risk of renal stone formation, and should therefore be avoided if possibte.

\section{Loteratery Tests}

There ore no known infarctions of Toparax topiramote with commonly used labontony tests.

Use in Prognency and lactation

like other ontiepileptic drugs, topiremate was teratogenic in mice, rots, ond rabbits. In rots, topiromate cosses the plocentrob bastier.

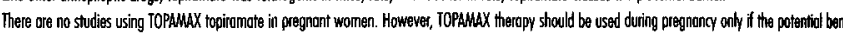
efit outweights the potential risk to the fatus.

Topiromate is excreted in the millk of hactoting rots. It is not known if topiramate is excreted in hurnon milk. Since many drugs ore excreted in humon milk

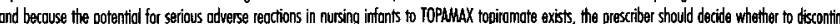
ue nursing or discontinue the drug, toking into account the risk / benefit ratio of the impartence of the druyg to the mother and the risks to the intant.

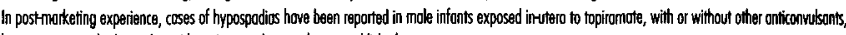
however, o cousal relationship with topiramate has not been estrablished.

The effect of TOPAMAX topiranate on labour ond delivery in humans is unknown.

Podiatiric Use

Soffety and effectiveness in children under 2 yours of oge hava not been estoblishod.

Geriatric Use

There is limited infornation in patients' over 65 years of oge. The possibility of ageassocioted renol function obnormalifies should be considered when using TOPAMAX topiromote.

\section{Roce and Gender Effecls}

Athough direct comporisen studies of pharmncokinetics have not been conducted, anolysis of phessmo concentration dato from clinical afficocy trids have

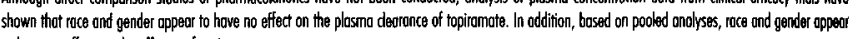
to heve no effect on the efficas of topiramate.

Adults

\section{ADVERSE REACTIONS}

The most conmmonly observed adverse events ossocicited with the adjunctive use of TOPAMAX topiramote of dosoges of 200 to $400 \mathrm{~mm} / \mathrm{doy}$ in controteded triak in ad ults that were seen at greoter frequency in topiromote-treated potients ond did not appear to be dose relatad within tis dosage ronge were: som nolence, dizziness, otoxio, speech disorders ond ralatad speech problems, psychomotor slowing, nystogmus, and pcresthesin (sae Tablo 2).

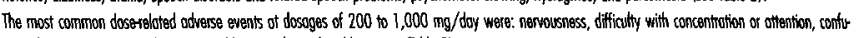
sion, depression, onorexia, languoge problems, ond mood problems (see Toble 3). Table 2

Incidence of Treatmentfrmergent Adverse Events in Placebo-fontrolled, Add-On Trials in ADULTS

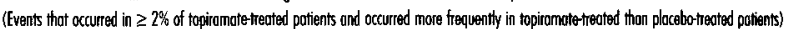

\begin{tabular}{|c|c|c|c|}
\hline \multirow[b]{2}{*}{ Body System/ } & \multicolumn{3}{|c|}{ 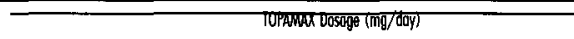 } \\
\hline & Placebo & $200-400$ & $600-1,000$ \\
\hline
\end{tabular}

Adverse tyent

Body as a Whold

Asthenio

Back Pain
Chest Poin

Chest Poin
Influenzo-Like Symptoms

Log Poin
Hot Flushes

Hot Flushes

$(\mathrm{n}=215)$

$(n=4)$

Dizziness

Speech Oisorders/Relatet Speedh Problems

Nystrginus

Poresthesi

Longuoget Problems

Coondination Abnomal

Hypooesthesia

Gashointestinal System

Nouser

Dyspepsio

Adoominal Pain

Constipotion

Mry Mouth

Weight Decreose

Neurropsychiatric

Somnolence

Psychomotor Slowing

Nenousness

Dificulty with Memory

Contusion

Depression

Difficully with Concentration/Altention

Anorexio

Agitation

Agoressive Reaction

Apotily

Depersonalization

Emotionol Lability

Reproductive, Fomalo

Breast Poin, Femol

Dysmenounibo

Menstnol Disorder

Reproductivo, Mulo

Rospitic Disorder

Respitotory
Pharyngitis

Pharyngits
Rhinitis

Rhinitits
Sinusitis

Sinusitis
Dysprea

Skin and Appendages

Prunitus

Vision

Diplapio
Vision Abnorm

Vision Abnormal

White Coll

1.4
4.2
2.8
3.2
2.3
1.9

8.0
6.2
4.4
3.5
3.5
2.7

15.3
6.9

3

6

.5

0.9
1.4

21
16.
15
15
10
6.2
5.3
2.7
1.

.5

3.7

0.9

28

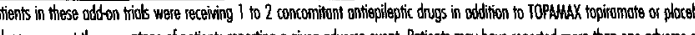

Values represent the percentage of potients reporting a given adverse event. Patients may have reperted more thon one adverse event during the study and can be included in more than one adverse event category.

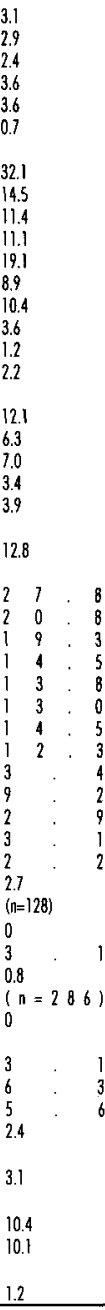


Toble 3

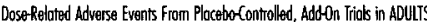

\begin{tabular}{|c|c|c|c|c|}
\hline \multirow[b]{2}{*}{ Adverso Event } & \multirow[b]{2}{*}{$\begin{array}{c}\text { Placebo } \\
\langle\mathrm{n}=216)\end{array}$} & \multicolumn{3}{|c|}{ TOPAMAX Dosoge (mg/doy) } \\
\hline & & $\begin{array}{c}200 \\
(n=45)\end{array}$ & $\begin{array}{c}400 \\
(n=68)\end{array}$ & $\begin{array}{r}600-1,000 \\
(n=414)\end{array}$ \\
\hline Fotigue & 13.4 & 11.1 & 11.8 & 29.7 \\
\hline Nervousnesss & 7.4 & 13.3 & 17.6 & 19.3 \\
\hline $\begin{array}{l}\text { Difficully with } \\
\text { Concentration/Atrention }\end{array}$ & 1.4 & 6.7 & 8.8 & 14.5 \\
\hline Contusion & 4.2 & 8.9 & 10.3 & 13.8 \\
\hline Depression & 5.6 & 8.9 & 7.4 & 13.0 \\
\hline Anorexio & 3.7 & 4.4 & 5.9 & 12.3 \\
\hline Language problems & 0.5 & 2.2 & 8.8 & 10.1 \\
\hline Anxioty & 6.0 & 2.2 & 2.9 & 10.4 \\
\hline Mood problemis & 1.9 & 0.0 & 5.9 & 9.2 \\
\hline
\end{tabular}

In six double-blind dinical triols, $10.6 \%$ of subiects (n=113) assigned to a topiramote dosacoge of 200 to $400 \mathrm{mg} /$ doy in oddition to their stondard AED ther

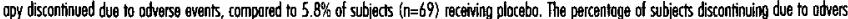

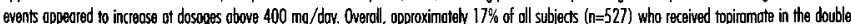
bind tiints discontinuad dua to atverse events, compored to $4 \%$ of the subiects (n=216) receiving ploceto.

Pediatriks

Adverse events ossociated with the use of topiramote of dosages of 5 to $9 \mathrm{mg} / \mathrm{kg} /$ day in worldwide patiotic clinical trials that were seen of greater fre quency in topiomate-reatad patients were: fotigue, somnolence, anorexio, nervousness, difficulty with concentrotion/attention, difficulty with memory aggressive reaction, ond weight decreosse.

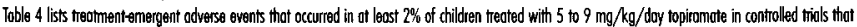
were numerically mose conmmon thon in patients trested with placeto

Toble 4

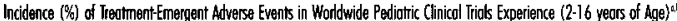

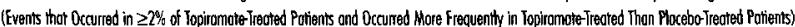

\begin{tabular}{|c|c|c|}
\hline $\begin{array}{l}\text { Body System/ } \\
\text { Advarse Event }\end{array}$ & $\begin{array}{l}\text { Placebla } \\
(N=101)\end{array}$ & $\begin{array}{l}\text { Topiramate } \\
\text { (N=98) }\end{array}$ \\
\hline \multicolumn{3}{|l|}{ Body as a Whoto - Gomeral Disorders } \\
\hline Fatigue & 5 & 16.3 \\
\hline Iniury & 12.9 & 14.3 \\
\hline Allergic Reaction & 1 & 2 \\
\hline \multicolumn{3}{|c|}{ Contral \& Peripheral Mervous System Disordors } \\
\hline Goit Abnormol & 5 & 8.2 \\
\hline Ataxio & 2 & 6.1 \\
\hline Hyperkinesio & 4 & 5.1 \\
\hline Dizziness & 2 & 4.1 \\
\hline Speech Disordars/Reloted Speech Problems & 2 & 4.1 \\
\hline Convulsions Aggravoted & 3 & 3.1 \\
\hline Hyporefloxio & 0 & 2 \\
\hline \multicolumn{3}{|l|}{ Gustrointestinel Systom Disordors } \\
\hline Nouseo & 5 & 6.1 \\
\hline Solvo Increosed & 4 & 6.1 \\
\hline Constipotion & 4 & 5.1 \\
\hline Gostroententitis & 2 & 3.1 \\
\hline \multicolumn{3}{|l|}{ Metabelk end Nutritionel Disorders } \\
\hline Weight Decreasso & 1 & 9.2 \\
\hline Thist & 1 & 2 \\
\hline \multicolumn{3}{|l|}{ Platelet, Bloediling \& Clotting Disordors } \\
\hline Purpura & 4 & 8.2 \\
\hline Epistaxis & 1 & 4.1 \\
\hline \multicolumn{3}{|l|}{ Mervous Disordars } \\
\hline Somnalencer & 15.8 & 25.5 \\
\hline Апогехxi & 14.9 & 24.5 \\
\hline Nervousnass & 6.9 & 14.3 \\
\hline Personality Disorder (Beihavior Problentis) & 8.9 & 11.2 \\
\hline Difiticully with Concentration/Attention & 2 & 10.2 \\
\hline Aggressive Reoction & 4 & 9.2 \\
\hline linsomnio & 6.9 & 8.2 \\
\hline Mood Problems & 6.9 & 7.1 \\
\hline Difficully with Mannory MOS: & 0 & 5.1 \\
\hline Emotional lobility & 5 & 5.1 \\
\hline Confusion & 3 & 4.1 \\
\hline Psychomotor Slowing & 2 & 3.1 \\
\hline \multicolumn{3}{|l|}{ Reproductive Disorders, Fomale } \\
\hline Loukorheo & 0.0 & 2.3 \\
\hline \multicolumn{3}{|l|}{ Rosistunce Moctenism Disorders } \\
\hline Infection Virol & 3.0 & 7.1 \\
\hline Infection & 3.0 & 3.1 \\
\hline \multicolumn{3}{|l|}{ Respiratory System Disorders } \\
\hline Upper Respiratary lract Infection & 36.6 & 36.7 \\
\hline Preumonia & 1.0 & 5.1 \\
\hline \multicolumn{3}{|l|}{ Skin and Apposdages Disorders } \\
\hline Skin Disorder & 2.0 & 3.1 \\
\hline Alopecia & 1.0 & 2.0 \\
\hline Dermalitis & 0.0 & 2.0 \\
\hline Hypertrichosis & 1.0 & 2.0 \\
\hline Rosh Enythematous & 0.0 & 2.0 \\
\hline \multicolumn{3}{|l|}{ Urinary System Disorders } \\
\hline Urinory Incontinences & 2.0 & 4.1 \\
\hline \multicolumn{3}{|l|}{ Vision Disordhrs } \\
\hline Eye Abnormality & 1.0 & 2.0 \\
\hline Vision Abnormal & 1.0 & 2.0 \\
\hline \multicolumn{3}{|l|}{ Whilie Call and RES Disordors } \\
\hline Leukoperio & 0.0 & 2.0 \\
\hline
\end{tabular}

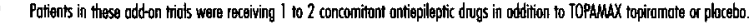

Volues sepresent the percentage of patients reporting o given adverse event. Patients may hove reportad more than one adverse event during the study ond can be indudeded in more than one adverse event carbegory.

Not Otharwise Spoctied

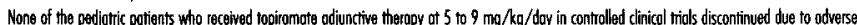
events. In open extensions of the controlled dinical trials, approximotely 9\% of the 303 peadiatic patients who ceceived topiramate of dosages up to $30 \mathrm{mg} / \mathrm{kg} /$ doy discontinued due to odverse events. Adverse events associoted with discontinuing therropy included aggravoted convulsions $(2.3 \%)$, larguage problems (1.3\%), and dificiculy with concentrtetion/antention $(1.3 \%)$.

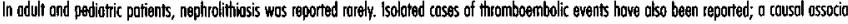
tion with the duvg hos not been estroblishod.

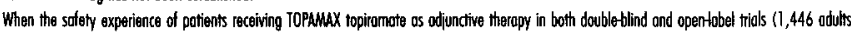
and 303 children) was analyzed, a similor paltern of adverse eyents emerged.
Pest-Merketing Adverse Reactions

The most frequenty reportad odverse events in sponttoneous post-manketing reports on topiromate include:

Psychiatrice somnolence or sedation, hallucinotion(s), depression, anorerexio, oggressive reoction, asychosis, thinking abnormol, poranoid reaction, insomnia, emotional lobility, suicide ontempt, delusion

Contral and Peripheral Nervous System: confusion, convulsions aggrovotod, poresthesio, agitotion, speech disordet, otoxio, diziness, convut sions, omnesio, heodoche, hyperkinesio

Metabolit and Nulritionat: weight deccease

Antonomk Nervous Systeme vomiting

Vision: vision obnormal

Gostrointestinak nuusso, dianhea, dbdominol poin, constipotion

Body as a Whole - General Disorders: fotigue

Urimary System: renal calculus

Skin and Appendages: rosh

\section{SYMPTOMS AND TREATMENT OF OVERDOSAGE}

In acute TOPAHAX topiromote overdose, if the ingestion is recent, the stomach should be emptiad immediataly by lovage or by industion of emesis. Activated charcoal has not been shown to adsorb topiramate in vitio. Thereforose, its use in overdosoge is not recommended. Fradtment should be oppropriately supportive.

Hemodialysis is on effective means of removing topiramete trom tha body. However, in the few cosses of ocute overdosoge reported, indluding doses of over $20 \mathrm{~g}$ in one individual, hemadialysis hos not bogn nacessary

\section{DOSAGE AND ADMUMISTRATION}

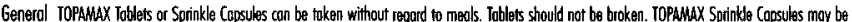

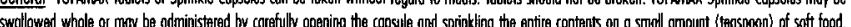
This drug/food mixture should be swallowed immediately ond not chewed. It shoult not be stored for future use. The sppinkle formulation is provided for This drug/food mixture should be swallowed immediately ond not chawe.

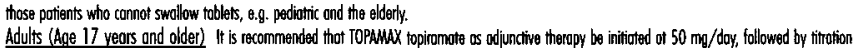
as needed and tolerated to on effective dose. At weekly intervals, the dose may be increased by $50 \mathrm{mg} /$ doy ond token in two divided doses. Some patients may benefit from lower inital doses, e.g. $25 \mathrm{mg}$ ond/or a slower litration schedule. Some potients may achieve efficocy with once-a-doy dosing.

The recormmended totol doily mointenance dose is $200 \mathrm{mg}-400 \mathrm{mg} /$ doy in two divided doses. Doses above $400 \mathrm{mg} / \mathrm{doy}$ hove not been shown to improve responses ond have been associoted with a greater incidence of odverse events. The moximum recommended dose is $800 \mathrm{mg} /$ doy. Daily doses above $1,600 \mathrm{mg}$ hrove not been studied.

Children (Ages 2-16 vears) It is recommended that TOPAMax topiramate as adjivactive theropy be initioted of $25 \mathrm{mg}$ (or less, based on a ronge of 1 to

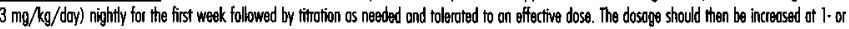
2-week intervals by increments of 1 to $3 \mathrm{mg} / \mathrm{kg} / \mathrm{doy}$ (odministared in nwo divided doses). Some potients may benefit form lower initial doses and/or o slower titration scheddle.

The recommended total daily maintenance dose is approxirnataly 5 to $9 \mathrm{mg} / \mathrm{kg} /$ doy in two divided doses. Dally doses up to $30 \mathrm{mg} / \mathrm{kg} /$ doy hove beetn studiad ond were gennerolly well toleroted.

Geriourics

Soe PRECAUTIONS section.

Patients with Renal Impairment

In renally impaired subjects (creatinine cloaronce less then $70 \mathrm{~mL} / \mathrm{min} / 1.73 \mathrm{~m}^{2}$ ), onehaff of the usuel odult dose is recommended. Such patients will require a longer time to rench steadystrtite at each dose.

Patients Undergoing Henodidysis

Topiramote is deared by hemodialysis at a rote that is 4 to 6 times greater than a normal individual. Accordinghy, o probonged period of dialysis may couse topiromote concentration to foll below that required to maintain on ontiseizure effect. To avoid rapid drops in topiramate phosmo concenntrotion during

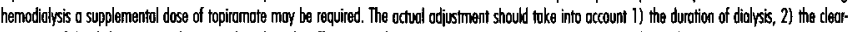

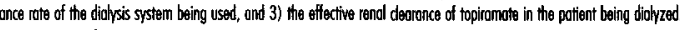

Patients with Hepotic Disease

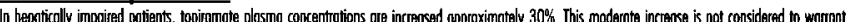

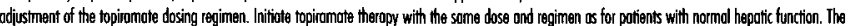
dose tirction in these potients should be guided by clinical outcame, i.e. seizure control, and avoithonce of adverse fffects. Such patients will require o longer tine to reach steadystate of each dose.

\section{AVALLABILITY OF DOSAGE FORMS}

TOPAMAX topiramote is ovoiloble as embossed foblets in the following strengths as described below:

$25 \mathrm{mg}$ : white, round, cooted toblets containing $25 \mathrm{mg}$ topirannate.

$100 \mathrm{mg}$ : yellow, round, cooted toblets contining $100 \mathrm{mg}$ topiromate.

$200 \mathrm{mg}$ : solmonc-oloured, round, cooted toblets contoining $200 \mathrm{mg}$ topiromate.

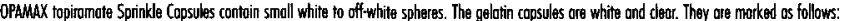

$15 \mathrm{mg}$ : "TOP" and "15 mg" on the side.

$25 \mathrm{mg}$ "TOP" ond "25 mg" on the side.

Supplied: Bontiles of 60 tablets with desiccent.

Bottles of 60 copsules without desiccont

TOPAMaX is o Schodule F Drig.

Product Monograph avialable to physicions and pharmacists upon requesst.

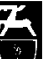

JANSSEN-ORTHO Inc.

Jonssen-Ortha Ic.,Joronto, Ontroris M3C II9

Date of Issuance: April 2000

IXP1001013A

() 2000 JANSSEN-ORTHO In

* All trodemork rights used under license

Rember

PAAB

Suppl. 2 - S83 


\section{Risperdal $\sqrt{ }$}

BRIEF PRESCRIBING INFORMATION

CONSULT FULL PRODUCT MONOGRAPH

FOR COMPLETE PRESCRIBING INFORMATION

"RISPERDAL*

ispenidone tablets

risperidone tartrate oral solution

risperidone $1 \mathrm{mg} / \mathrm{mL}$

Antipsychotic Agent

\section{CLINICAL PHARMACOLOGY}

\section{BEHAVIOURAL DISTURBANCES IN SEVERE \\ DEMENTIA}

The effect of RISPERDAL upon the management of behavioural disorders in geriatric patients with severe dementia was evaluated in two clinical trials. The first study was a fixed dose, dose-response study in which RISPERDAL, at daily doses of $0.5,1.0$ and $2.0 \mathrm{mg}$ per day, was compared to placebo $(\mathrm{N}=147$ to 162 per group). The second study was a flexible dose stud in which RISPERDAL was compared to HALDOL and placebo $(\mathrm{N}=114$ to 115 per group). The duration of the studies was 12 weeks. In both studies, patients had to meet the DSM-IV criteria for Alzheimer's, and/or vascular dementia. The scales used to assess symptomatic efficacy included the BEHAVE-AD (Behavioural Pathology in Alzheimer's Disease Rating Scale), the CMAI (Cohen-Mansfield Agitation Inventory) and the CGI-C (Clinical Global Impression-Change). Potential extrapyramidal adverse events were assessed by the ESRS (Extrapyramidal Symptom Rating Scale).

In the dose-response study, $73 \%, 16 \%$ and $12 \%$ of patients were diagnosed with Alzheimer's, vascular and mixe dementia, respectively. At baseline, the MMSE (Mini-Menta State Examination) scores ranged from 6.0 to 7.8 , and more than $95 \%$ of patients were at least at stage 6 on the FAST (Functional Assessment Staging). The median ages of the RISPERDAL-treated patients ranged from 82 to 84 years with an overall range of 60 to 105 years. RISPERDAL, 1.0 and $20 \mathrm{mg}$ per day, given b.i.d., decreased significantly both verbal and physical aggression and psychotic behaviour. The differences between the $0.5 \mathrm{mg}$ dose and placebo did not reach statistical significance. The incidence of extrapyramidal adverse events was significantly higher with RISPERDAL, 20 mg per day, than with placebo. The difference between the lower doses and placebo was not significant.

In the flexible dose study, $67 \%, 26 \%$ and $7 \%$ of patients were diagnosed with Alzheimer's, vascular and mixed dementia, respectively. At baseline, the MMSE scores ranged from 7.9 to 8.8 , and $61 \%$ and $31 \%$ of patients were at stage 6 and stage 7 on the FAST, respectively. The median age of the RISPERDALtreated patients was 81 years (range 68 to 97 years).

RISPERDAL, at a mean endpoint dose of $1.1 \mathrm{mg}$ per day, given b.i.d., decreased significantly aggressive behaviour but not psychosis. ESRS scores, assessing extrapyramidal symptoms, were similar in RISPERDAL and placebo-treated patients. RISPERDAL had no effect on any of the other behaviours assessed by the BEHAVE-AD, namely activity disturbances, anxieties and phobias, or affective disturbances. Furthermore the drug had no effect on either the MMSE scores or the FAST.

\section{INDICATIONS AND CLINICAL USE}

\section{RISPERDAL (risperidone) is indicated for the management of}

the manifestations of schizophrenia and related psychotic disorders. In controlled clinical trials, RISPERDAL was found to improve both positive and negative symptoms of schizophrenis. RISPERDAL may be useful in severe dementia for the shortterm symptomatic management of inappropriate beh to aggression and/or psychosis. Other behavioural disturbances seen in this patient population as well as disease stage remained unaffected by RISPERDAL treatment (see

\section{Clinical Studies)}

\section{CONTRAINDICATIONS}

icated in patients with a known hypersensitivity to the drug or the excipients of the

\section{WARNINGS}

During clinical trials, RISPERDAL (risperidone) has been observed to cause orthostatic hypotension and tachycardia, especially during the initial dose titration period and the first few weeks of treatment. Rare cases of syncope, cardiac arrhythmias and first degree AV-block have been reported The likelihood of excessive hypotension or syncope can be minimized by limiting the initial dose of the drug to

$1-2 \mathrm{mg}$ per day, q.d. or b.i.d., in adult patients and to 0.25 to $0.5 \mathrm{mg}$ b.i.d. in special patient populations, and by increasing the dose slowly (see DOSAGE AND ADMINISTRATION). A the dose slowly (see DOSAGE AND ADMINISTRATION). A
dose reduction should be considered if hypotension occurs. Patients with a history of clinically significant cardiac disorders were excluded from clinical trials. Therefore, RISPERDAL should be used with caution in patients with cardiovascular diseases (e.g., heart failure, history of myocardial infarction or ischemia, cerebrovascular disease, conduction abnormalities and other conditions such as dehydration and hypovolemia. Special care should be taken to avoid hypotension in patients with a history of cerebrovascular insufficiency or ischemic heart disease, and in patients taking medications to lower blood pressure.

Neuroleptic Malignant Syndrome (NMS)

Neuroleptic malignant syndrome is a potentially fatal symptom complex that has been reported in association with antipsychotic drugs, including RISPERDAL

Clinical manifestations of NMS are hyperthermia, muscle rigidity, altered mental status (including catatonic signs) and evidence of autonomic instability lirregular blood pressure, tachycardia, cardiac arrhythmias, and diaphoresis). Additional signs may include elevated creatine phosphokinase. myoglobinuria (rhabdomyolysis), and acute renal failure. In arriving at a diagnosis, it is important to identify cases where the clinical presentation includes both serious medical illnes (e.g., pneumonia, systemic infection, etc.) and untreated or inadequately treated extrapyramidal signs and symptoms. Other important considerations in the differential diagnosis include central anticholinergic toxicity, heat stroke, drug fever, and primary central nervous system pathology.

The management of NMS should include: 1) immediate discontinuation of all antipsychotic drugs including RISPERDAL and other drugs not essential to concurrent therapy; 2) intensive symptomatic treatment and medical monitoring; and 3) treatment of any concomitant serious medical problems for which specific treatments are available. There is no general agreement about specific pharmacological treatment regimens for uncomplicated NMS.

If a patient requires antipsychotic drug treatment after recovery from NMS, the potential reintroduction of drug therapy should be carefully considered. The patient should be carefully monitored, since recurrence of NMS has been reported.

Tardive Dyskinesia (TD

A syndrome consisting of potentially irreversible, involuntary, dyskinetic movements may develop in patients treated with conventional antipsychotic drugs. Although TD appears to be most prevalent in the elderly, especially elderly females, it is impossible to predict at the onset of treatment which patients are likely to develop TD.

It has been suggested that the occurrence of parkinsonian side effects is a predictor for the development of TD. In clinical studies, the observed incidence of drug-induced parkinsonis was lower with RISPERDAL than with haloperidol. In the optimal clinical dose range, the difference between risperidone and haloperidol was significant. The risk of developing TD may be less with RISPERDAL

The risk of developing TD and the likelihood that it will become irreversible are believed to increase as the duration of treatment and the total cumulative dose of antipsychotic drugs administered to the patient increase. However, the syndrome can develop, although less commonly, after relatively brief periods of treatment at low doses. There is no known treatment for established cases of TD. The syndrome may remit, partially or completely, if antipsychotic drug treatment is withdrawn. However, antipsychotic drug treatment itself may suppress the signs and symptoms of TD, thereby masking the underlying process. The effect of symptom suppression upon the long-term course of TD is unknown.

In view of these considerations, RISPERDAL should be prescribed in a manner that is most likely to minimize the risk of TO. As with any antipsychotic drug, RISPERDAL should be reserved for patients who appear to be obtaining substantial benefit from the drug. In such patients, the smallest dose and the shortest duration of treatment should be sought. The need for continued treatment should be reassessed periodically.

If signs and symptoms of TD develop during treatment with RISPERDAL, withdrawal of the drug should be considered However, some patients may require treatment with RISPERDAL despite the presence of the syndrome.

\section{PRECAUTIONS}

\section{Potential Effect on Cognitive and Motor Performance}

Since RISPERDAL (risperidone) may cause somnolence and impair motor skills, patients should be cautioned against driving a car or operating hazardous machinery until they are reasonably certain that RISPERDAL does not affect them adversely.

Seizures

Antipsychotic drugs are known to lower the seizure threshold. In clinical trials, seizures have occurred in a few RISPERDALtreated patients. Therefore, caution should be used in administering RISPERDAL to patients having a history of seizures or other predisposing factors.

Antiemetic Effect

Consistent with its dopamine antagonistic effects, RISPERDAL may have an antiemetic effect. Such an effect may mask signs of toxicity due to overdosage with other drugs, or may mas symptoms of disease such as brain tumor or intestinal obstruction.

Body Temperature Regulation

Disruption of the body's ability to reduce core body temperature has been attributed to antipsychotic drugs. Appropriate care is advised when prescribing RISPERDAL for patients who will be experiencing conditions which may contribute to an elevation of core temperature, e.g., exercising strenuously, exposure to extreme heat, receiving concomitant medication with anticholinergic activity, or being subject to dehydration

Suicid

The possibility of suicide or attempted suicide is inherent in psychosis, and thus, close supervision and appropriate clinica management of high-risk patients should accompany drug therapy.

Priapism

This cases of priapism have been reported with RISPERDAL This adverse reaction, as with other psychotropic drugs, did not appear to be dose-dependent and did not correlate with the duration of treatment. The most likely mechanism of priapism is a relative decrease in sympathetic tone.

Drug Interactions

The risk for potential interaction between RISPERDAL and other drugs has not been evaluated systematically. Given the primary central nervous system effects of RISPERDAL, caution should be used when it is taken in combination with other centrally acting drugs and alcohol. Because of its potential for inducing hypotension, RISPERDAL may enhance the hypotensive effects of other therapeutic agents. RISPERDAL may antagonize the effects of levodopa and dopamine agonists. Carbamazepine has been shown to decrease substantially the plasma levels of risperidone and its active metabolite,

9-hydroxy-risperidone. Similar effects may be observed with other hepatic enzyme inducers. Consequently, in the presence of carbamazepine or other hepatic enzyme inducers the dose of RISPERDAL may have to be adjusted. On discontinuation of these drugs, the dosage of RISPERDAL should be re-evaluated and, if necessary, decreased.

The effect of RISPERDAL $(0.5 \mathrm{mg} /$ day administered b.i.d.) on

the steady state plasma concentrations of digoxin
$(0.125 \mathrm{mg} /$ day) was examined in a double-blind, two-way, $(0.125 \mathrm{mg} /$ day) was examined in a double-blind, two-way,
crossover trial in 19 healthy elderly volunteers (median age 68 years, range 61 to 75 years). RISPERDAL did not affect the steady-state pharmacokinetics of digoxin, and concurre administration of the two drugs was well tolerated.

The metabolism of RISPERDAL, a substrate of the hepatic cytochrome P450 isozyme 206 (CYP 2D6), is affected by the debrisoquine hydroxylation polymorphism. CYP $2 \mathrm{D} 6$ is also responsible for the metabolism of a variety of drugs, includin phenothiazines, antidepressants (tricyclics and SSRIs). antiarrhythmics and some B-blockers. Consequently, potential interaction between RISPERDAL and drugs that are also interaction between RISPERDAL and drugs that ar
substrates of CYP 2D6, should also be considered.

In vitro studies, in which risperidone was given in the presence of various, highly protein-bound agents, indicated that clinically relevant changes in protein binding would not occur either for RISPERDAL or for any of the drugs tested.

Hyperprolactinemia

Antipsychotic drugs elevate prolactin levels with the effect persisting during chronic administration. In controlled clinical trials, RISPERDAL elevated substantially serum prolactin levels: in female patients, mean levels ranged between 48 and $57 \mathrm{ng} / \mathrm{mL}$ at doses ranging from 4 to $16 \mathrm{mg}$ per day. The prolactin levels were considerably higher in RISPERDAL-treated patients than in haloperidol-treated patients.

Since tissue culture experiments indicate that approximately one-third of human breast cancers are prolactin dependent in vitro, RISPERDAL should only be administered to patients with previously detected breast cancer if the benefits outweigh the potential risks. Caution should also be exercised when considering RISPERDAL treatment in patients with pituitary
tumours. Possible manifestations associated with elevated prolactin levels are amenorrhea, galactorrhea, and menorrhagia (see ADVERSE REACTIONS).

In carcinogenicity studies, the administration of risperidone resulted in an increase in the incidence of mammary neoplasms in both rats and mice. In addition, adenomas of the endocrine pancreas in male rats and pituitary adenomas in female mice have been noted. These changes have been attributed to elevated prolactin levels and have also been observed with other dopamine receptor antagonists. To date, neither clinical studies nor epidemiological studies have show an association between chronic administration of these drugs and mammary tumorigenesis.

Weight Gain

With continued treatment, weight gain (mean: $2.3 \mathrm{~kg}$ in long. term studies) has been seen.

Use in Pregnancy

The safety of RISPERDAL during pregnancy has not been established. In animal studies, risperidone did not show direct reproductive toxicity. However, due to its prolactin elevating and CNS depressant activities, reproductive performance and pup survival were adversely affected in rats. Risperidone was not teratogenic in either rats or rabbits.

RISPERDAL should not be used during pregnancy uniess the expected benefits to the patient markedly outweigh the potential risks to the fetus

Use During Lactation

It is not known whether RISPERDAL is excreted in human milk. Risperidone appeared in the milk of lactating dogs. The concentration of risperidone was similar in milk and plasma, while that of 9-hydroxy-risperidone was higher in the milk than 
Use in Children

The safety and efficacy of RISPERDAL in children under the age of 18 have not been established.

Use in Patients with Hepatic Impairment

Although the pharmacokinetics of RISPERDAL in patients with hepatic impairment were comparable to those in young volunteers, the free fraction of risperidone was increased. Since this may lead to a more pronounced pharmacologica effect, lower starting doses and lower maximal doses are recommended in these patients (see DOSAGE AND ADMINISTRATION).

Use in Patients with Renal Impairment

The pharmacokinetics of RISPERDAL were significantly altered in patients with renal disease. Since clinical experience is lacking in this patient population, dosage recommendations cannot be made at this time.

\section{Use in Patients with Parkinson's Disease}

Use in Patients with Parkinson's Disease RISPERDAL, ike other dopamine antagonists, may cause patients and

should therefore be used with caution.

\section{ADVERSE REACTIONS}

\section{SCHIZOPHRENIA AND RELATED}

Adverse Events Associated with Discontinuation of Treatment Adverse Events Associated with Discontinuation of Treatment An estimated $9 \%$ of approximately 1800 patients who discontinued treatment due to adverse reactions. The more common events causing discontinuation included: Psychiatric (4.1\%): primarily psychosis, agitation, suicide attempt, somnolence; Neurological (3.2\%): primarily extrapyramidal disorder, dizziness; and Cardiovascular (1.2\%): primarily hypotension, Other events leading to discontinuation included: tachycardia/palpitations $(0.6 \%)$, nervousness $(0.4 \%)$, nausea $(0.3 \%)$ and insomnia $(0.3 \%)$.

Commonly Observed Adverse Events in Short-term Clinical Trials

The most frequent adverse reactions observed during clinical trials with RISPERDAL, at doses of $\leq 10 \mathrm{mg} /$ day, were insomni $(13 \%)$, agitation $(9 \%)$, extrapyramidal disorder $(7 \%)$, anxiety

$(7 \%)$, headache $(6 \%)$ and rhinitis $(3 \%)$. In some instances it has been difficult to differentiate adverse events from symptoms of the underlying psychosis.

the undertying psychosis
Serious Adverse Events

The most serious adverse reactions were rare cases of

The most serious adverse reactions were rare cases of
syncope, cardiac arrhythmias, first degree AV-block, and syncope,
seizures.

Extrapyramidal Symptoms

Parkinsonian side effects were usually mild but dose-related they were reversible upon dose reduction and/o administration of antiparkinsonian medication.

Vital Sign Changes

Hypotension (including orthostatic), and tachycardia (including reflex tachycardia) have been observed following the administration of RISPERDAL (see WARNINGS).

ECG Changes

Electrocardiograms were evaluated in RISPERDAL-treated $(\mathrm{N}=380)$. HALDOL-treated ( $N=126)$ and placebo-treated $(\mathrm{N}=120)$ patients. In the RISPERDAL group, eight patients had a slight increase in QTc intervals from less than $450 \mathrm{msec}$ at baseline to intervals ranging from 450 to 474 msec during treatment. Changes of this type were not seen in placebo-treated patients but were observed in three HALDOL-treated subjects. Hyperprolactinemia

Hyperprolactinemia
RISPERDAL elevated plasma prolactin levels. Associated RISPERDAL elevated plasma prolactin levels. Associate
manifestations, namely amenorrhea, galactorrhea, and manifestations, namely amenorrhagia have occurred

Wenorrhagia

Weight Gain placebo in the treatment of schizophrenia, 18\% of RISPERDALtreated patients and $9 \%$ of placebo-treated patients met a weight gain criterion of $\geq 7 \%$ of baseline body weight. Thi difference was statistically significant.

\section{Other Adverse Events}

Erectile dysfunction, ejaculatory dysfunction, orgastic dysfunction, and rash have also been observed during treatment with RISPERDAL. In one study, in which testosterone levels were measured, testosterone decreased below the normal range in 6 out of 85 patients. As with other antipsychotics, cases of water intoxication, either due to polydipsia or to inappropriate secretion of antidiuretic hormone (ADH), have occasionally been reported during treatment with RISPERDAL.

For additional details regarding adverse events in controlled clinical trials in schizophrenia, consult the Product Monograph Adverse Reactions During Long-Term Treatment

Long-term treatment with RISPERDAL was carried out in 386 chronic schizophrenic patients, with 213 patients receiving the drug for at least one year. The UKU side effect rating scale was used to elicit adverse events.

Listed (in decreasing order) are those events which showed deterioration during treatment compared to baseline in at least $10 \%$ of patients. Psychic: asthenia/lassitude/increased fatiguability, concentration difficulties, sleepiness/sedation, reduced duration of sleep, increased duration of sleep, failin memory, increased dream activity; Autonomic: orthostatic dizziness, constipation, nausea/vomiting, polyuria/polydipsia palpitations/tachycardia, reduced salivation, accommodatio disturbances, increased tendency to sweating, diarrhea; Other: weight gain, weight loss, amenorrhea, ejaculatory dysfunction, erectile dysfunction, diminished sexual desire, tension headache, increased sexual desire, orgastic dysfunction.
Postmarketing

International postmarketing reporting revealed the following adverse drug reactions during RISPERDAL treatment: edema, increased hepatic enzyme levels, skin manifestations of allergy including a case of Stevens-Johnson syndrome, systemic manifestations of allergy including a case of anaphylactic shock, neuroleptic malignant syndrome and rare cases of tardive dyskinesia, hypertension, leukopenia, and priapism. Rarely, mild to moderate neutropenia associated in a few cases with thrombopenia has been reported. To date, a causal relationship to risperidone has not been established. As with other neuroleptics, sudden deaths have been reported durin RISPERDAL treatment. Most of the patients hed pre-existing cardionatisting to risperidone has not been established at this time.

\section{BEHAVIOURAL DISTURBANCES}

\section{IN SEVERE DEMENTIA}

Adverse Events Associated with Discontinuation of Treatment In the fixed dose, dose-response study, 95/617 patients discontinued treatment due to an adverse event. The most frequently reported adverse events were somnolence, extrapyramidal symptoms (EPS), and agitation, with extrapyramidal symptoms (EPS), and agit.
somnolence and EPS being dose-related.

\begin{tabular}{|c|c|c|c|c|}
\hline \multirow[t]{2}{*}{ Adverse Event } & \multirow{2}{*}{$\begin{array}{c}\text { Placebo } \\
\text { (N=161) } \\
\%\end{array}$} & \multicolumn{3}{|c|}{ RISPERDAL } \\
\hline & & $\begin{array}{c}0.5 \mathrm{mg} / \text { day } \\
(\mathrm{N}=147) \\
\%\end{array}$ & $\begin{array}{c}1 \mathrm{mg} / \mathrm{day} \\
(\mathrm{N}=147) \\
\%\end{array}$ & $\begin{array}{c}2 \mathrm{mg} / \mathrm{day} \\
(\mathrm{N}=162) \\
\%\end{array}$ \\
\hline Somnolence & 1.9 & 0 & 4.8 & 6.8 \\
\hline $\begin{array}{l}\text { Extrapyramidal } \\
\text { symptoms (EPS) }\end{array}$ & 1.2 & 1.4 & 3.4 & 3.7 \\
\hline Agitation & 2.5 & 2.0 & 1.4 & 3.7 \\
\hline
\end{tabular}

Incidence of Adverse Events

Table 2 enumerates adverse events from the fixed dose, doseresponse study that were more frequent in the RISPERDAL

groups than in the placebo group and/or were dose-related.

\begin{tabular}{|c|c|c|c|c|}
\hline \multirow{2}{*}{$\begin{array}{l}\text { Body System/ } \\
\text { Preferred Term }\end{array}$} & \multirow{2}{*}{$\begin{array}{l}\text { Placebo } \\
\text { (N }=161 \text { ) } \\
\%\end{array}$} & \multicolumn{3}{|c|}{ RISPERDAL } \\
\hline & & $\begin{array}{c}0.5 \mathrm{mg} / \mathrm{day} \\
(\mathrm{N}=147) \\
\%\end{array}$ & $\begin{array}{c}1 \mathrm{mg} / \text { day } \\
\text { (N=147) } \\
\%\end{array}$ & $\begin{array}{c}2 \mathrm{mg} / \text { day } \\
(\mathrm{N}=162) \\
\%\end{array}$ \\
\hline $\begin{array}{l}\text { Body as a Whole } \\
\text { Edema peripheral }\end{array}$ & 6 & 16 & 13 & 18 \\
\hline $\begin{array}{l}\text { Psychiatric } \\
\text { Somnolence }\end{array}$ & 8 & 10 & 17 & 27 \\
\hline $\begin{array}{l}\text { Neurological } \\
\text { Extrapyramidal } \\
\text { symptoms (EPS) }\end{array}$ & 8 & 7 & 13 & 22 \\
\hline $\begin{array}{l}\text { Respiratory } \\
\text { Rhinitis } \\
\text { Dyspnea }\end{array}$ & $\begin{array}{l}5 \\
1\end{array}$ & ; & ${ }_{1}^{6}$ & $\begin{array}{c}10 \\
5\end{array}$ \\
\hline $\begin{array}{l}\text { Cardiovascular } \\
\text { Hypotension } \\
\text { Tachycardia }\end{array}$ & $\begin{array}{l}3 \\
1\end{array}$ & $i_{1}^{2}$ & $\begin{array}{l}3 \\
0\end{array}$ & $\begin{array}{l}5 \\
2\end{array}$ \\
\hline
\end{tabular}

Other adverse events, which occurred with a high incidence but with similar frequencies in the RISPERDAL- and placebotreated patients included injury $(28$ to $38 \%$ ), falls (13 to $25 \%)$, urinary tract infection (13 to $21 \%$ ), and purpura (10 to 17\%).

\section{DOSAGE AND ADMINISTRATION}

RISPERDAL (risperidone) may be given as tablets or or solution and may be taken with or without meals. In order to avoid orthostatic hypotension, the dose of RISPERDAL should be adjusted gradually.

\section{SCHIZOPHRENIA AND}

\section{REITTTD PSYCHOTIC DISORDERS}

Adults

RISPERDAL can be administered on either a q.d. or b.i. schedule, generally beginning with 1 to $2 \mathrm{mg}$ per day. The dose should be adjusted gradually over several days based on clinical response to a target dose of 4 to $6 \mathrm{mg}$ per day. Some patients may benefit from lower initial doses and/or a slower adjustment schedule.

Further dosage adjustments, if indicated, should generally occur at intervals of not less than one week since steady state for the active metabolite would not be achieved for

approximately one week in the typical patient. When dosage adjustments are necessary, small increments/decrements of $1 \mathrm{mg}$ are recommended.

In controlled clinical trials, optimal therapeutic effects were seen in the 4 to $8 \mathrm{mg}$ per day dose range. However, clinical experience indicates that in the majority of patients adequate therapeutic effect is achieved at the $6 \mathrm{mg}$ per day dose. Doses above $10 \mathrm{mg}$ per day have not been shown to be more efficacious than lower doses and were associated with more extrapyramidal symptoms and other adverse events.

The safety of RISPERDAL has not been established above $16 \mathrm{mg}$ total daily dose, administered twice daily. If administered once daily, safety has not been established beyond a single dose of 8 $\mathrm{mg}$.

In elderly schizophrenic patients, the doses of RISPERDAL should be adjusted slowly from a $0.25 \mathrm{mg}$ b.i.d. starting dose to a maximum daily dose of $3 \mathrm{mg}$. Since the elimination of RISPERDAL is somewhat slower in these patients, the potentia for accumulation should be considered.

Patients Prone to Hypotension

Caution should be exercised in patients prone to hypotension and the use of lower starting doses of 0.25 to $0.5 \mathrm{mg}$ b.i.d. should be considered.

Patients with Impaired Liver Function

Although the pharmacokinetics of RISPERDAL, in response to a $1 \mathrm{mg}$ single dose, did not change in patients with impaired live function, the free fraction of risperidone was increased and this may result in an enhanced effect. Consequently, in patients with impaired liver function, the starting dose should be 0.25 to $0.5 \mathrm{mg}$ b.i.d. This dosage can be individually adjusted in $0.5 \mathrm{mg}$ b.i.d. increments to 1 to $2 \mathrm{mg}$ b.i.d.

Patients with Impaired Kidney Function

Since the pharmacokinetics of RISPERDAL changed

substantially in patients with renal disease, even in response to a $1 \mathrm{mg}$ single dose (see PRECAUTIONS), and since to date no clinical experience is available, dosage recommendations cannot be made in this patient population.

Switching From Other Antipsychotics

When medically appropriate, gradual discontinuation of the previous treatment, while RISPERDAL therapy is initiated, is recommended. In all cases, the period of overlapping antipsychotic administration should be minimized. Whe switching patients from depot antipsychotics, initiate RISPERDAL therapy in place of the next scheduled injection. The need for continuing existing antiparkinsonian medications should be re-evaluated periodically.

\section{BEHAVIOURAL DISTURBANCES IN SEVERE \\ DEMENTIA}

Elderly

In the elderly with severe dementia, careful dosage adjustment and low doses are recommended.

A starting dose of RISPERDAL $0.25 \mathrm{mg}$ b.i.d. is recommended This dosage should be adjusted by increments of $0.25 \mathrm{mg}$ per day approximately every 2 to 4 days. The optimal dose is 0.5 $\mathrm{mg}$ b.i.d. $(1.0 \mathrm{mg}$ per day) for most patients. Some patients, however, may benefit from higher doses up to a maximum of $1.0 \mathrm{mg}$ b.i.d. (2.0 mg per day).

Periodic dosage adjustments (increase or decrease) or discontinuation of treatment should be considered because of the instability of the symptoms treated.

Since there is no experience in younger patients, dosage recommendations cannot be made.

\section{AVAILABILITY OF DOSAGE FORMS}

T

Tablets

$0.25 \mathrm{mg}$ risperidone as yellow, oblong, biconvex tablets (marke JANSSEN and Ris 0.25 ); in blister packages of 60 tablets.

$0.5 \mathrm{mg}$ risperidone as brownish-red, half-scored, oblong, biconvex tablets (marked JANSSEN and Ris 0.5); in blister packages of 60 tablets.

$1 \mathrm{mg}$ risperidone as white, film-coated, oblong tablets (marked JANSSEN and $R$ 1): in blister packages of 60 tablets and HDPE bottles of 250 tablets. Tablets may be scored

$2 \mathrm{mg}$ risperidone as orange, film-coated, oblong tablets (marked JANSSEN and R 2); in blister packages of 60 tablets and HDPE bottles of 250 tablets.

$3 \mathrm{mg}$ risperidone as yellow, film-coated, oblong tablets (marke JANSSEN and $R 3$ ); in blister packages of 60 tablets and HDPE bottles of 250 tablets.

$4 \mathrm{mg}$ risperidone as green, film-coated, oblong tablets (marked JANSSEN and R 4): in blister packages of 60 tablets and HDPE bottles of 250 tablets.

Oral Solution

RISPERDAL $1 \mathrm{mg} / \mathrm{mL}$ oral solution is supplied in $100 \mathrm{~mL}$ bottles with a plastic child resistant closure and a calibrated (in milligrams and millilitres) pipette. The minimum calibrated volume is $0.25 \mathrm{~mL}$, while the maximum calibrated volume is 3 $\mathrm{mL}$

Patient Instructions (including illustrations) for using the RISPERDAL calibrated dispensing pipette are provided with the packaging. Tests indicate that RISPERDAL oral solution is compatible in the following beverages: water, coffee, orange juice and low-fat milk. However, it is NOT compatible with cola or tea.

\section{RISPERDAL is a Schedule F drug}

Product Monograph available to physicians and pharmacists upon request.

\section{Janssen-0rtho Inc}

Toronto, Ontario

$\mathrm{M} 3 \mathrm{C}$

Date of Issuance: August 1999

Component t: PIRI991014A

- All trademark rights used under license 